\title{
Calibrations for charged particle tracking and measurements of $\omega$ photoproduction with the GlueX detector
}

\author{
by \\ Michael Justin Staib \\ Submitted in partial fulfillment of the \\ requirements for the degree of \\ Doctor of Philosophy \\ at \\ Carnegie Mellon University \\ Department of Physics \\ Pittsburgh, Pennsylvania
}

Advised by Professor Curtis Meyer

September 21, 2017 


\begin{abstract}
The GlueX experiment is a new experimental facility at Jefferson Lab in Newport News, VA. The experiment aims to map out the spectrum of hybrid mesons in the light quark sector. Measurements of the spin-density matrix elements in $\omega$ photoproduction are performed with a linear polarized photon beam on an unpolarized proton target, and presented in bins of Mandelstam $t$ for beam energies of $8.4-9.0 \mathrm{GeV}$. The spin-density matrix elements are exclusively measured through two decays of the $\omega$ meson: $\omega \rightarrow \pi^{+} \pi^{-} \pi^{0}$ and $\omega \rightarrow \pi^{0} \gamma$. A description of the experimental apparatus is presented. Several methods used in the calibration of the charged particle tracking system are described. These measurements greatly improve the world statistics in this energy range. These are the first results measured through the $\omega \rightarrow \pi^{0} \gamma$ decay at this energy. Results are generally consistent with a theoretical model based on diffractive production with Pomeron and pseudoscalar exchange in the $t$-channel.
\end{abstract}




\section{Acknowledgments}

After so many years of effort towards the culmination of this work, it is hard to come up with an exhaustive list of people who have helped me along the way. I feel like I've missed someone, so if that is you, I'm sorry, and thanks for your help. I'd like to thank my advisor, Curtis Meyer, for his useful scientific advice and for showing me the best way to wear a bowtie. Reinhard Schumacher has also provided many valuable suggestions in times I have been short on ideas. I'd also like to thank Gregg Franklin and Brian Quinn for their valuable input in preparing the final version of this document. Thanks to Marcus Hohlmann for giving me a chance to prove myself in the field. Thanks to Kim Skinner and Bob Jester for teaching me years ago that science can be fun. I've learned that sometimes that is true.

The GlueX collaboration has been an excellent group of scientists to work with, and I will always remember the excitement of participating in the commissioning of the experiment (with the exception of labeling the BCAL LEMO cables). I'd first like to apologize to Sean Dobbs, Paul Mattione, and Justin Stevens for my oft rambling emails about one problem or another, but thanks for your friendship and advice at odd hours. You and the other postdocs all helped make my graduate years at CMU a little easier by taking on a lot of the hard work. Thanks to Mike Williams for his help in preparing the code that implements the theoretical model presented in this thesis and for setting the bar too high with his previous analysis on the subject. I'd like to thank Dave Mack for always providing extensive feedback on my analyses, and his general enthusiasm and insightful intuition into arcane matters. I'd like to thank Beni Zihlmann for indirectly teaching me some colorful SwissGerman phrases among other more useful things. I'd like to thank Lubomir Pentchev for his help calibrating the FDC, and for always making me explain myself in more detail. Maybe another person that deserves more apologies than thanks is Simon Taylor, for helping me understand the tracking code used in the experiment and for being a sounding board for my calibration ruminations. Thanks to David Lawrence for guiding me through some of my more technical problems with the software. Many, many thanks to Nick Sandoval, Chris Stanislav and the rest of the techs at JLab that actually make an experiment like GlueX possible and keep the physicists from breaking things (Beni, don't touch the CDC). Most all of the Hall D staff at JLab has helped me along the way, so thanks to Eugene, Elton, Mark, Mark, Hovanes, Alexandre, Sasha and Sergei for our useful discussions through my tenure with the experiment. I'd also like to thank Matt Shepherd and Richard Jones for their help and suggestions through the years.

When not obsessed with some problem plaguing the experimental reconstruction, my friends have always helped me relax. Thanks to Matt, Nora, Zach, Will ${ }^{2}$, Patrick, Naomi, Mark, Rachelle, Brad, Brian, Chasen, Juan Carlos, Larisa, Alexa, and Abel for always being great friends and colleagues in Pittsburgh. Thanks to Eric, Jenn, Alex, Alex, Anja, Ray, John, Matt, Trevor, and Malia for making VA a little more bearable when I was there.

Most importantly, I'd like to acknowledge the constant support of my family. This work is dedicated to them for allowing me to choose my own path in life that lead me here. To my family: enjoy reading my thesis, but I totally understand if you don't make it that far ;-). 


\section{Contents}

1 Introduction 1

1.1 Quantum Chromo-Dynamics . . . . . . . . . . . . . . . . . . 1

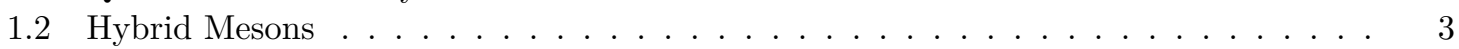

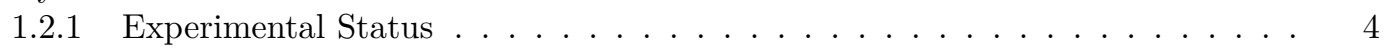

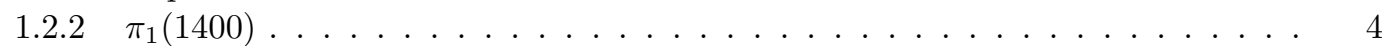

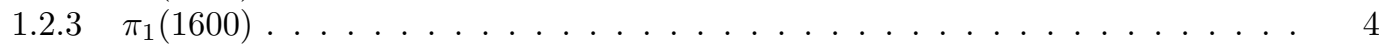

1.3 Exclusive $\omega$ Photoproduction $\ldots \ldots \ldots \ldots \ldots \ldots \ldots \ldots$

1.4 Spin-Density Matrix Elements in $\omega$ Photoproduction . . . . . . . . . . . . 7

1.4.1 Photon Density Matrix _. . . . . . . . . . . . . . . . 8

1.4.2 Vector Meson Spin-Density Matrix . . . . . . . . . . . . . . . . 8

1.4.3 Angular Distributions . . . . . . . . . . . . . . . . . . . . 9

1.5 The Oh, Titov, Lee Model . . . . . . . . . . . . . . . . . . . . . 11

2 The GlueX Detector $\quad 15$

2.1 Photon Beamline . . . . . . . . . . . . . . . . . . . . . . . 15

2.1 .1 Photon Tagger . . . . . . . . . . . . . . . . . . . . . . . 16

2.1 .2 Triplet Polarimeter . . . . . . . . . . . . . . . . . . . . . . . . 16

2.1 .3 Pair Spectrometer . . . . . . . . . . . . . . . . . . . . . 20

2.2 Liquid Hydrogen Target . . . . . . . . . . . . . . . . . . . . . . . . . . . 22

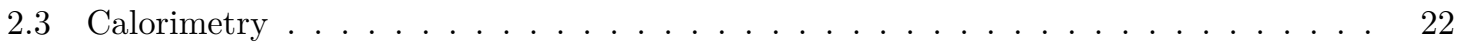

$2.3 .1 \quad$ Barrel Calorimeter . . . . . . . . . . . . . . . . . . . . 23

2.3.2 Forward Calorimeter . . . . . . . . . . . . . . . . . . . 24

2.4 Charged Particle Tracking . . . . . . . . . . . . . . . . . . . . . . 24

2.4 .1 Central Drift Chamber . . . . . . . . . . . . . . . . . . . 25

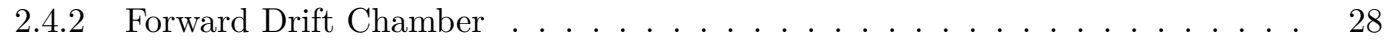

2.5 Particle Identification Detectors . . . . . . . . . . . . . . . . . . . . . . . 29

$2.5 .1 \quad$ Start Counter . . . . . . . . . . . . . . . . . . . . . . . . 29

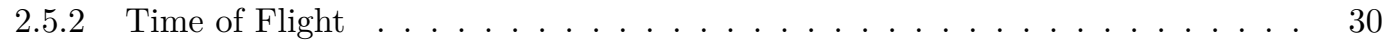

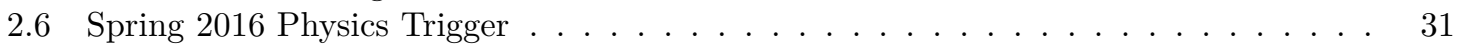

3 Charged Particle Tracking Calibrations $\quad 34$

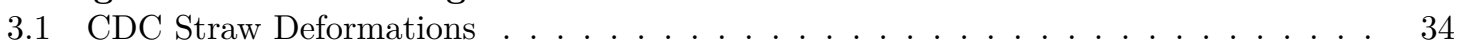

3.2 Internal FDC Alignment . . . . . . . . . . . . . . . . . . . . . . . . . . . 39

3.3 Track-Based Alignment . . . . . . . . . . . . . . . . . . . . . . 44

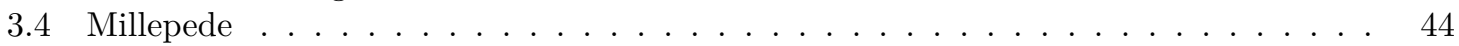

3.4 .1 General Overview . . . . . . . . . . . . . . . . . 44

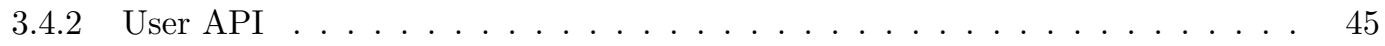

3.4 .3 Millepede for GlueX . . . . . . . . . . . . . . . . . . . . 46

3.4.4 Millepede Alignment Procedure . . . . . . . . . . . . . . . . . . . . 48 
3.4.5 Results using Millepede for Alignment of GlueX . . . . . . . . . . . . . . . 49

4 Event Selection $\quad \mathbf{6 1}$

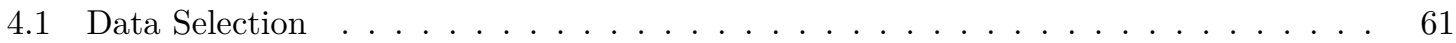

4.2 Data Processing . . . . . . . . . . . . . . . . . . . . . . . 62

4.3 Assembly of Particle Combinations . . . . . . . . . . . . . . . . . . . . . . . 62

4.3.1 Beam Photon Selection .................... 62

4.3 .2 Kinematic Fitting . . . . . . . . . . . . . . . . . 63

4.4 Additional Exclusivity Cuts . . . . . . . . . . . . . . . . . . . . . . . 64

4.4.1 Measured Missing 4-Momenta . . . . . . . . . . . . . . . . . 64

4.4 .2 Measured $\pi^{0}$ Mass . . . . . . . . . . . . . . . . . . . . . . . . . . . . . . . . 68

4.4 .3 Vertex Location . . . . . . . . . . . . . . . . . . . . . . 69

4.4 Measured Photon Kinematics . . . . . . . . . . . . . . . . . . . . 69

4.4.5 Particle Identification Based on $\mathrm{dE} / \mathrm{dx} \ldots \ldots . . . . . . . . . . .770$

4.4.6 Particle Identification Based on Time-of-Flight . . . . . . . . . . . . . . . . 71

4.4.7 Summary of Analysis Cuts . . . . . . . . . . . . . . . . . . . . 72

4.5 Combinations Passing Analysis Cuts . . . . . . . . . . . . . . . . . . . . 72

$4.5 .1 \omega$ Invariant Mass . . . . . . . . . . . . . . . . . . . . . . . . . . . . . . . . . . . . . . . . . . . . . . .

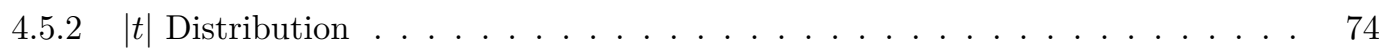

5 Measurement of Spin Density Matrix Elements $\quad 76$

5.1 Likelihood Fit . . . . . . . . . . . . . . . . . . . . . . . . . . . 76

5.2 Monte Carlo Sample for Acceptance Correction . . . . . . . . . . . . . . . . . . . . 78

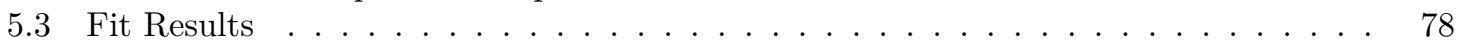

5.4 Systematics Studies . . . . . . . . . . . . . . . . . . . . 83

5.5 Discussion of Results . . . . . . . . . . . . . . . . . . . . . . . 86

$\begin{array}{lr}\text { A SDME Fit Results } & 95\end{array}$

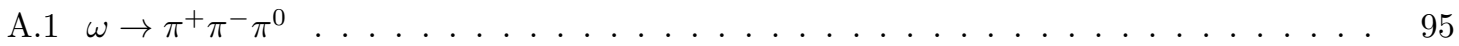

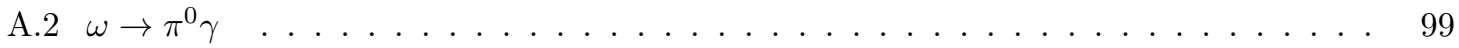

B Table of Systematic Uncertainties $r$ 


\section{Chapter 1}

\section{Introduction}

The GlueX experiment aims to sharpen our understanding of the dynamics of the universe on the shortest length scales. Recent measurements of the structure of mesons described by the theory of Quantum Chromo-Dynamics (QCD) show tantalizing hints of the rich structure of the underlying theory. The GlueX experiment will measure light meson properties using a linearly polarized photon beam, and will provide valuable input to models describing the strong interaction.

\subsection{Quantum Chromo-Dynamics}

The interactions we intend to explore in this thesis are mediated by the strong force. By measuring these production processes, it is possible to extract information about the underlying theory. The interaction of quarks through exchanged gluons is described by the theory of QCD. These quarks and gluons have a property known as "color" that can be understood as the $\mathrm{SU}(3)$ analog of the electrical charge in the theory of Quantum Electro-Dynamics (QED). QCD famously predicts asymptotic freedom of the quarks, where at short distances the quarks behave as if they are free [1]. As distance increases, the interaction between quarks gets stronger resulting in confinement of the quarks. Due to this confinement, we are unable to detect free quarks in nature. What we do observe are colorless combinations of two or three quarks known respectively as mesons and baryons.

A plot of the coupling strength of the theory versus the energy scale is shown in Figure 1.1. At very high energy, the coupling strength decreases to the point that the theory is computable by standard perturbative methods. At lower energy, as is the case for the GlueX experiment, the theory becomes highly nonlinear and can not be solved perturbatively. Other methods must be employed to extract predictions from the model. One such method to provide predictions from the theory in the nonperturbative regime is known as Lattice QCD.

In Lattice QCD, continuous Euclidean spacetime is discretized helping to regularize the theory of QCD. This lattice is a hypercube with a constant lattice spacing. Often in practice different spacing for the temporal and spatial dimensions are used. Quarks fields are placed at the lattice sites, and gluonic fields form the links between these locations on the lattice. Predictions in continuous spacetime are achieved in the limit that the lattice spacing goes to zero [2]. The masses of the light quarks are usually chosen to be heavier than physical quarks since lighter pions require a larger lattice to describe the physics and heavy mesons can decay to multiple pions [3]. A prediction for the isovector and isoscalar meson spectrum using lattice QCD is presented in Figure 1.2. In this figure, several of the predicted states can be interpreted as having more than just contributions from two valence quarks to make up the quantum numbers of the meson, and require contributions from the gluons themselves. These were not inserted into the calculation, but arise naturally from the structure of QCD. These states with excited gluons are know as "hybrid" mesons. The lightest hybrid mesons are outlined in orange in the figure. 


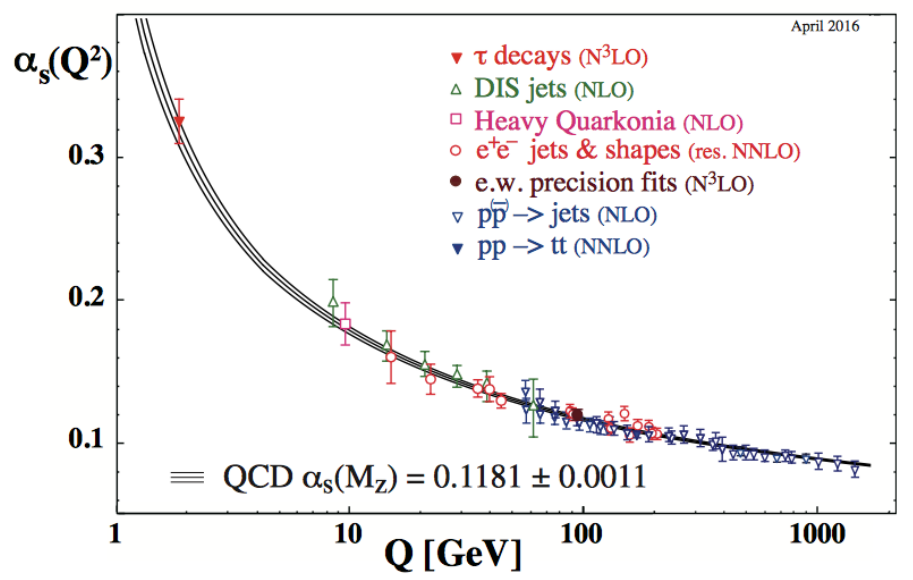

Figure 1.1: Summary of measurements of the strong coupling constant, $\alpha_{s}$, as a function of the energy scale. Reproduced from [2].

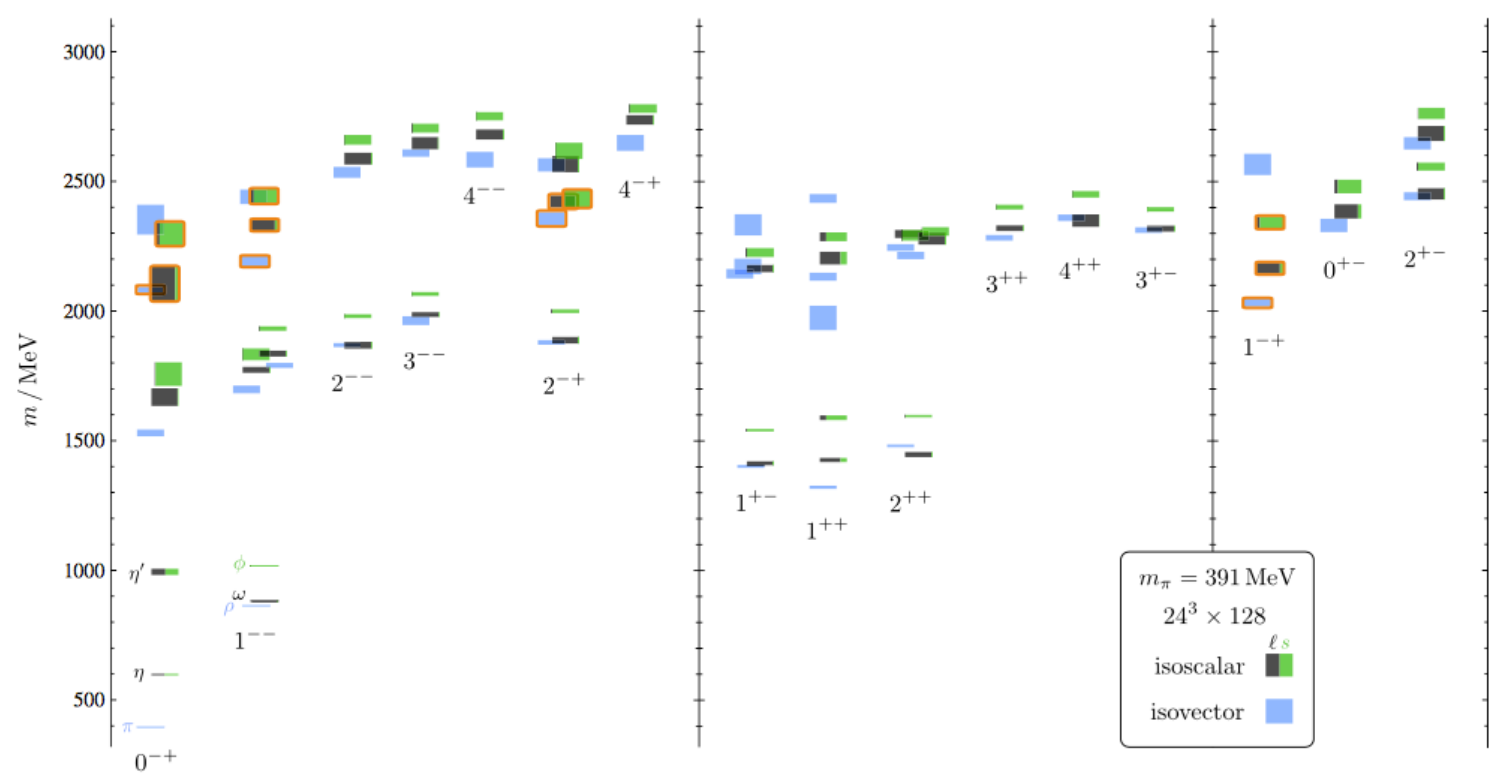

Figure 1.2: Spectrum of light meson predicted using Lattice QCD. The states outlined in orange are interpreted as the lightest hybrid mesons. Reproduced from [4]. 


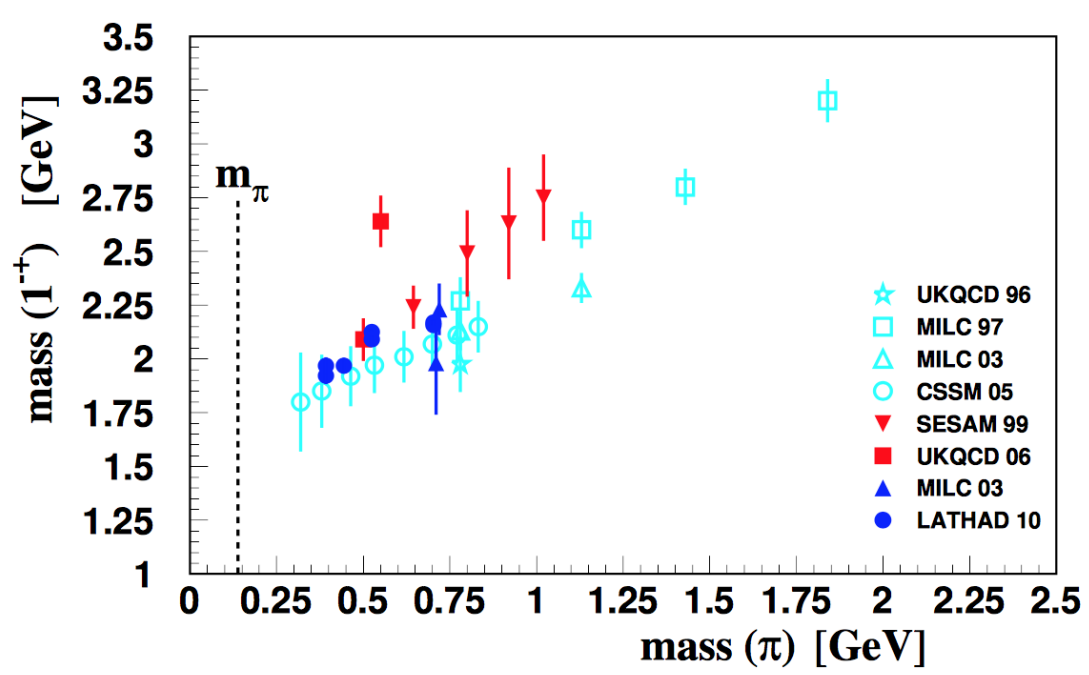

Figure 1.3: Lattice QCD predictions for the mass of the $1^{-+}$exotic meson as a function of the $\pi$ mass. Extrapolating to the physical pion mass predicts a $1^{-+}$mass of roughly $1.6 \mathrm{GeV}$. Reproduced from [3].

\subsection{Hybrid Mesons}

QCD predicts mesons that can not be explained by a simple $q \bar{q}$ model. Since these hybrid mesons are predicted, experimentalists should hopefully be able to detect them. This is made difficult by the fact that these particles decay to the same final states as much more copiously produced mesons. Thus it is impossible to detect these particles by simple bump hunting techniques since the backgrounds are high. However, a partial wave analysis may be employed to extract the individual components making up the total amplitude. One class of hybrid mesons - those with "exotic" quantum numbers - could be a clear indication that a hybrid state has been produced. States with these quantum numbers could appear in our partial wave decomposition.

The quark model picture of a standard meson is that of a $q \bar{q}$ pair. The individual quarks have spin-parity $J^{P}=1 / 2^{+}$, and the antiquarks $J^{P}=1 / 2^{-}$. The $q \bar{q}$ combination can then have intrinsic spin 0 or 1 depending on whether the spins are aligned or opposed. They may also have a relative orbital angular momentum $L$. The parity of these states is given by $P=(-1)^{L+1}$. For neutral light mesons, the eigenvalue under charge conjugation is given as $C=(-1)^{L+S}$. Therefore, if we look at the $L=0$ case, we find possible $q \bar{q}$ combinations with $J^{P C}=0^{-+}, 1^{--}$. If we increase to $L=1$ we can produce $J^{P C}=0^{++}, 1^{+-}, 1^{++}, 2^{++}$. By repeating this exercise, it can be shown that the quantum numbers $0^{--}, 0^{+-}, 1^{-+}, 2^{+-}, \ldots$ can not be produced by a standard $q \bar{q}$ pair and therefore they are known as exotic quantum numbers. If we allow the $1^{-+}$gluon to contribute to the quantum numbers as in a hybrid meson in a $q g \bar{q}$-like configuration, states with these quantum numbers can be reached. These exotic states are therefore likely hybrid in nature, making their detection one of the primary goals of the GlueX experiment. There are also hybrid mesons without exotic quantum numbers, but the extraction of the signal is made difficult by overlaps with standard $q \bar{q}$ resonances. Several lattice QCD predictions for the mass of the lowest lying $1^{-+}$state from various studies with different pion mass can be seen in Figure 1.3. If one projects these measurements to the physical pion mass, the prediction is for the lowest $1^{-+}$state to appear around $1.6 \mathrm{GeV}$.

An excellent resource describing the current landscape of experimental searches and theoretical predictions for hybrid mesons can be found in Reference [3]. A summary of the allowed decay modes of the lightest predicted exotic hybrid mesons can be found in Table 1.1. Each of the particles has 


\begin{tabular}{ccc}
\hline Name & $J^{P C}$ & Allowed Decay Modes \\
\hline$\pi_{1}$ & $1^{-+}$ & $b_{1} \pi, \pi \rho, \pi f_{1}, \pi \eta, \pi \eta^{\prime}, \eta a_{1}, \pi \eta(1295)$ \\
$\eta_{1}$ & $1^{-+}$ & $\pi a_{1}, \pi a_{2}, \eta f_{1}, \eta f_{2}, \pi \pi(1300), \eta \eta^{\prime}, K K_{1}^{A}, K K_{1}^{B}$ \\
$\eta_{1}^{\prime}$ & $1^{-+}$ & $K K_{1}^{B}, K K_{1}^{A}, K K^{*}, \eta \eta^{\prime}$ \\
$b_{0}$ & $0^{+-}$ & $\pi \pi(1300), \pi h_{1}, \rho f_{1}, \eta b_{1}$ \\
$h_{0}$ & $0^{+-}$ & $\pi b_{1}, \eta h_{1}, K K(1460)$ \\
$h_{0}^{\prime}$ & $0^{+-}$ & $K K(1460), K K_{1}^{A}, \eta h_{1}$ \\
$b_{2}$ & $2^{+-}$ & $\pi a_{1}, \pi a_{2}, \pi h_{1}, \eta \rho, \eta b_{1}, \rho f_{1}$ \\
$h_{2}$ & $2^{+-}$ & $\pi \rho, \pi b_{1}, \eta \omega, \omega b_{1}$ \\
$h_{2}^{\prime}$ & $2^{+-}$ & $K K_{1}^{B}, K K_{1}^{A}, K K_{2}^{*}, \eta h_{1}$ \\
\hline
\end{tabular}

Table 1.1: Summary of possible decay modes of exotic hybrid mesons. Reproduced from [3].

several possible decay modes. The GlueX experiment will be able to study multiple decay modes for each of these exotic candidates, with the goal of mapping out the spectrum of hybrid mesons.

\subsubsection{Experimental Status}

Through the years, there have been several reported observations of mesons with exotic quantum numbers appearing in the literature. These have primarily been of mesons with $J^{P C}=1^{-+}$, specifically $\pi_{1}(1400), \pi_{1}(1600)$ and the $\pi_{1}(2015)$. The signals are extracted by fitting the data to sets of partial waves of definite $J^{P C}$. This fit to partial waves is performed in bins of the invariant mass of the final state particles. The contributions of the $1^{-+}$wave to the total partial-wave fit can then be investigated. The coefficients on the amplitudes fit for in the partial-wave analysis are complex valued, so the phase motion of this wave relative to the other waves in the data set can be measured as well. Some of the measurements, particularly those of the $\pi_{1}(1600)$, are robust and measured in multiple decay channels across multiple experiments. However, the interpretation of these measurements as QCD hybrids is difficult since there are background processes besides true hybrid production that can fake the signal. GlueX will hopefully be able to help resolve some of these ambiguities in the interpretation of these measurements.

\subsection{2 $\pi_{1}(1400)$}

There are a number of experiments that have reported the observation of a resonance-like structure near $1.4 \mathrm{GeV}$ in the $1^{-+}$wave. The interpretation of the $\pi_{1}(1400)$ as the lightest exotic hybrid meson is questionable. Curiously, nearly all of these reported observations are in the $\eta \pi$ final state. This is unusual given the prediction of multiple decay modes for the hybrid mesons in Table 1.1. The resonance interpretation of the lone observation in the $\rho \pi$ final state at Obelix is also disputed [3]. In addition, the mass of the $\pi_{1}(1400)$ is lower than most theory predictions for the lightest exotic hybrid. It is likely that this state is in some way related to the dynamics of the production and is not a true resonance. A summary of many of the experimental observations of the $\pi_{1}(1400)$ is shown in Table 1.2 .

\subsection{3 $\pi_{1}(1600)$}

The $\pi_{1}(1600)$ is a much more likely hybrid candidate than the $\pi_{1}(1400)$. A plot showing the intensity of the $1^{-+}$wave in the VES experiment in the decay $X \rightarrow \eta^{\prime} \pi^{-} \rightarrow \eta \pi^{+} \pi^{-} \pi^{-}$is shown in Figure 1.4. There is clear enhancement around a mass of $1.6 \mathrm{GeV}$. There is also indication of phase motion of the $1^{-+}$wave relative to the $1^{++}$wave. VES sees this enhancement across multiple analyses and multiple decay modes. Measurements such as these provide hints that a state such as that predicted for hybrid mesons has been observed. A table of reported observations of the $\pi_{1}(1600)$ is presented 


\begin{tabular}{cccc}
\hline Decay Mode & Mass $[\mathrm{GeV}]$ & Width $[\mathrm{GeV}]$ & Experiment \\
\hline$\eta \pi^{-}$ & $1.405 \pm 0.020$ & $0.18 \pm 0.02$ & GAMS \\
$\eta \pi^{-}$ & $1.343 \pm 0.0046$ & $0.1432 \pm 0.0125$ & KEK \\
$\eta \pi^{-}$ & $1.37 \pm 0.016$ & $0.385 \pm 0.040$ & E852 \\
$\eta \pi^{0}$ & $1.257 \pm 0.020$ & $0.354 \pm 0.064$ & E852 \\
$\eta \pi$ & $1.40 \pm 0.020$ & $0.310 \pm 0.050$ & CBAR \\
$\eta \pi^{0}$ & $1.36 \pm 0.025$ & $0.220 \pm 0.090$ & CBAR \\
$\rho \pi$ & $1.384 \pm 0.028$ & $0.378 \pm 0.058$ & Obelix \\
\hline$\eta \pi$ & $1.354 \pm 0.025$ & $0.330 \pm 0.035$ & PDG Average \\
\hline
\end{tabular}

Table 1.2: Measurements of $\pi_{1}(1400)$ masses and widths reported in the literature. Reproduced from [3].
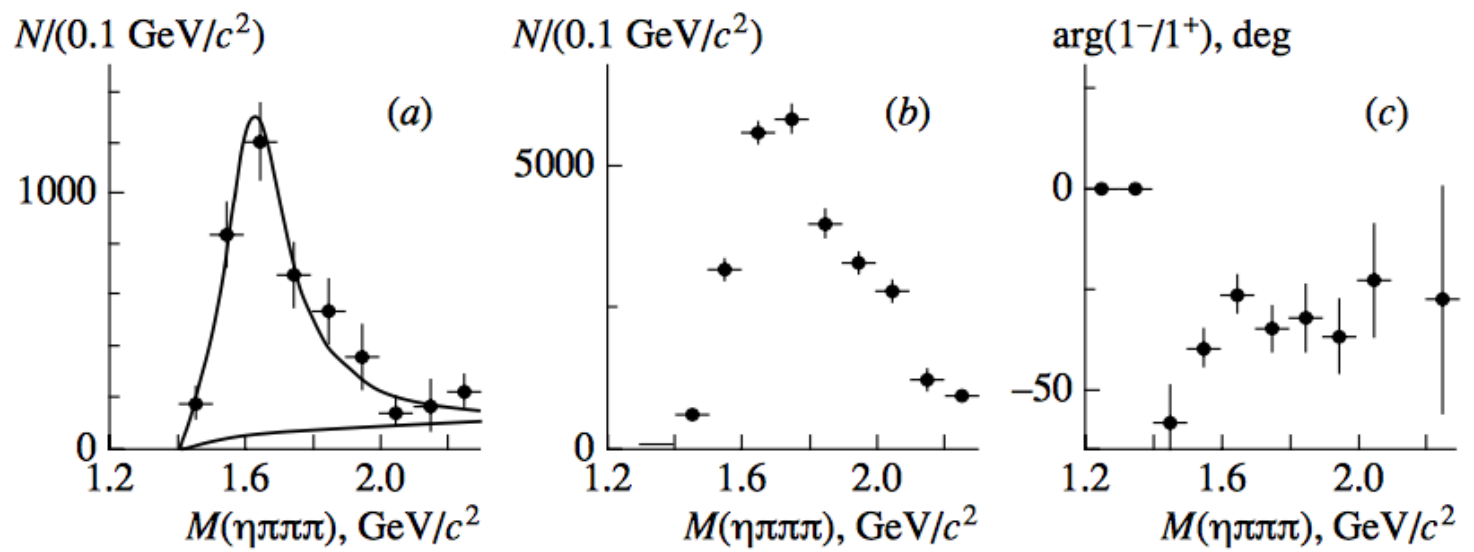

Figure 1.4: Results of partial wave analysis of the $\pi^{+} \pi^{-} \pi^{-} \eta$ system from VES. (a) Intensity of the $1^{++}$wave. (b) Intensity of the $1^{-+}$wave. (c) Phase difference between the two waves. Reproduced from $[5]$.

in Table 1.3. In this table we have not listed the measurements in the $\rho \pi$ channel since the results are questionable [3]. The results are consistent across multiple experiments and decay modes which lends well to the resonance interpretation of the state. It is likely that the $\pi_{1}(1600)$ is an actual resonance, but the unambiguous classification as a hybrid meson will require mapping out other members of the hybrid meson nonet. GlueX has been designed be able to contribute to this effort.

\subsection{Exclusive $\omega$ Photoproduction}

The search for exotic hybrid mesons with the GlueX detector will require a thorough understanding of the experimental apparatus. In order to reconstruct more complicated states, we need to show that we can measure basic photoproduction processes well. For example, the common decay of the $\pi_{1}(1600)$ to $b_{1} \pi$ is measured through the dominant decay $b_{1} \rightarrow \omega \pi$. In order to precisely measure this process, the experiment needs to demonstrate that it is capable of measurements of the $\omega$ meson. Exclusive $\omega$ photoproduction is one of the more copious reactions at GlueX photon energies of 8.4-9.0 GeV. The cross section for photoproduction of various hadronic final states versus beam energy is shown in Figure 1.5. Exclusive $\omega$ production makes up about 1-2\% of the total hadronic cross section, so it is an important process to study. Properties of the $\omega$ meson are found in Table 1.4 . 


\begin{tabular}{cccc}
\hline Decay Mode & Mass $[\mathrm{GeV}]$ & Width $[\mathrm{GeV}]$ & Experiment \\
\hline$b_{1} \pi$ & $1.58 \pm 0.03$ & $0.30 \pm 0.03$ & VES \\
$b_{1} \pi$ & $1.61 \pm 0.02$ & $0.29 \pm 0.03$ & VES \\
$b_{1} \pi$ & $1.56 \pm 0.06$ & $0.34 \pm 0.06$ & VES \\
$f_{1} \pi$ & $1.64 \pm 0.03$ & $0.24 \pm 0.06$ & VES \\
$\eta^{\prime} \pi$ & $1.58 \pm 0.03$ & $0.30 \pm 0.03$ & VES \\
$\eta^{\prime} \pi$ & $1.61 \pm 0.02$ & $0.290 \pm 0.03$ & VES \\
$\eta^{\prime} \pi$ & $1.56 \pm 0.06$ & $0.34 \pm 0.06$ & VES \\
$\eta^{\prime} \pi$ & $1.597 \pm 0.010$ & $0.340 \pm 0.040$ & E852 \\
$f_{1} \pi$ & $1.709 \pm 0.024$ & $0.403 \pm 0.080$ & E852 \\
$b_{1} \pi$ & $1.664 \pm 0.008$ & $0.185 \pm 0.025$ & E852 \\
$\eta^{\prime} \pi$ & $1.670 \pm 0.030$ & $0.240 \pm 0.050$ & CLEO-c \\
\hline$\eta \pi$ & $1.662_{-0.009}^{+0.008}$ & $0.241 \pm 0.040$ & PDG Average \\
\hline
\end{tabular}

Table 1.3: Measurements of $\pi_{1}(1600)$ masses and widths reported in the literature. Reproduced from [3].

\begin{tabular}{rcc}
\multicolumn{3}{c}{$\omega$ meson properties } \\
\hline$I^{G} J^{P C}$ & Mass & Width \\
\hline $0^{-} 1^{--}$ & $782.65 \pm 0.12 \mathrm{MeV}$ & $8.49 \pm 0.08 \mathrm{MeV}$ \\
& \\
Primary Decays & Branching Ratio \\
\hline$\pi^{+} \pi^{-} \pi^{0}$ & $89.2 \%$ \\
$\pi^{0} \gamma$ & $8.3 \%$ \\
$\pi^{+} \pi^{-}$ & $1.5 \%$
\end{tabular}

Table 1.4: Properties of the $\omega$ meson [2]. 


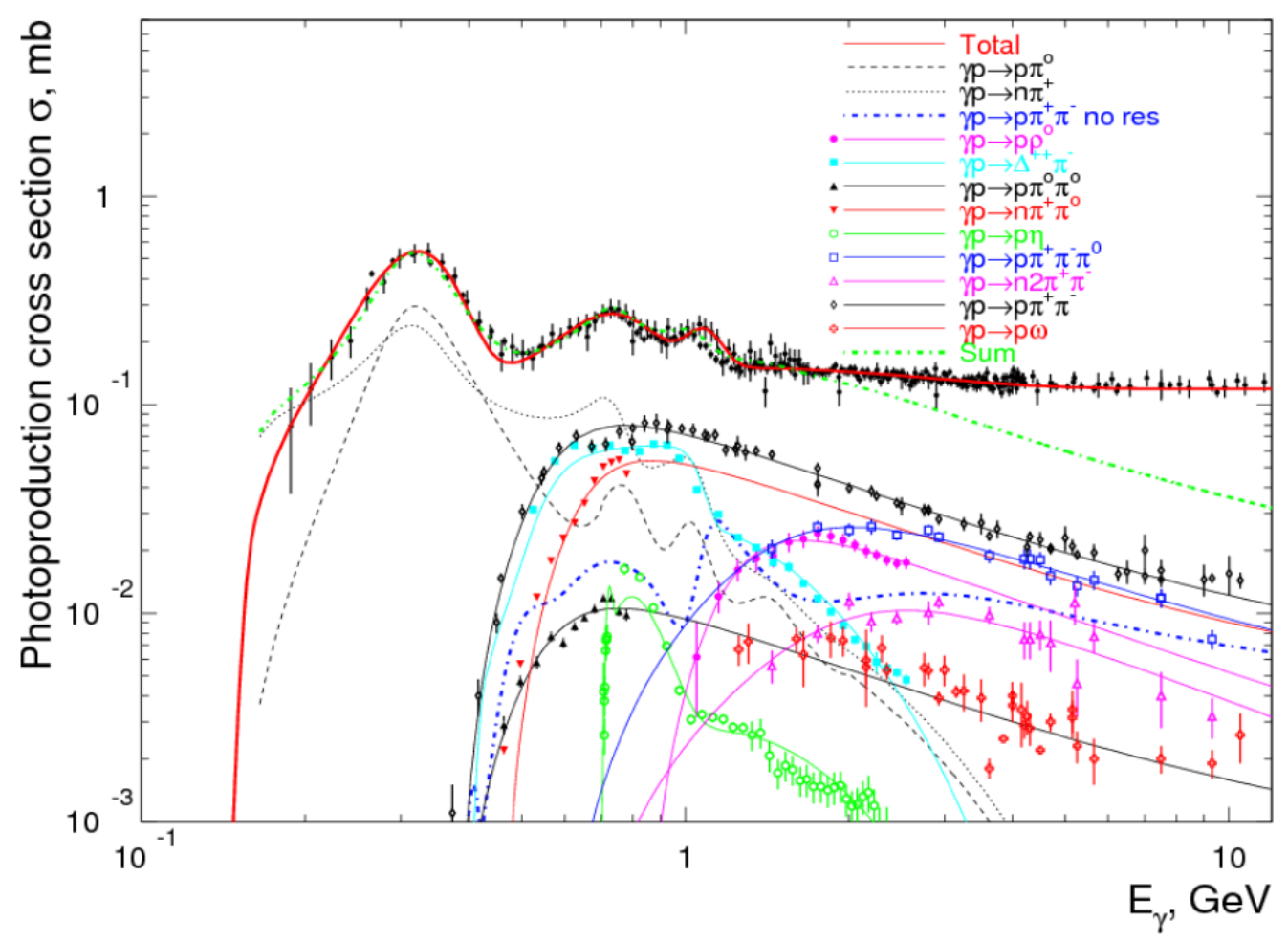

Figure 1.5: Cross sections for photoproduction versus photon energy. Reproduced from [6].

\subsection{Spin-Density Matrix Elements in $\omega$ Photoproduction}

By investigating the decay of $\omega$ mesons, it is possible to extract information about the production amplitudes through measurements of the Spin-Density Matrix Elements (SDMEs). These SDMEs are the bilinear covariants of the scattering theory description of the interaction $\gamma p \rightarrow \omega p$. Hence, they represent valuable information about the amplitudes themselves.

Here we follow a derivation of the SDMEs presented by Schilling, et al. [7]. We seek to describe the reaction

$$
\gamma N \rightarrow V N
$$

where a photon $\gamma$ is incident on a nucleon $N$ and produces a vector meson $V$. The spin configuration of a particle is described by the density matrix $\rho$. The production amplitudes $T$ relate the spin density of the incoming photon $\rho(\gamma)$ to that of the outgoing vector meson $\rho(V)$ at the vertex as

$$
\rho(V)=T \rho(\gamma) T^{\dagger}
$$

In the center of mass helicity basis, this may be written as

$$
\rho_{\lambda_{V} \lambda_{V}^{\prime}}(V)=\frac{1}{\mathcal{N}} \sum_{\lambda_{N^{\prime}} \lambda_{\gamma} \lambda_{N} \lambda_{\gamma}^{\prime}} T_{\lambda_{V} \lambda_{N^{\prime}}, \lambda_{\gamma} \lambda_{N}} \rho_{\lambda_{\gamma} \lambda_{\gamma}^{\prime}}(\gamma) T_{\lambda_{V}^{\prime} \lambda_{N^{\prime}}, \lambda_{\gamma}^{\prime} \lambda_{N}}^{*}
$$

where $\lambda$ indicates the helicity of each of particles in the reaction: $\lambda_{N} \in\{-1 / 2,1 / 2\}, \lambda_{V} \in\{-1,0,1\}$, $\lambda_{\gamma} \in\{-1,1\}$. The normalization factor $\mathcal{N}$ is given by

$$
\mathcal{N}=\frac{1}{2} \sum_{\lambda_{V} \lambda_{N^{\prime}} \lambda_{\gamma} \lambda_{N}}\left|T_{\lambda_{V} \lambda_{N^{\prime}} \lambda_{N} \lambda_{\gamma}}\right|^{2} .
$$




\subsubsection{Photon Density Matrix}

Consider a photon in its helicity basis. Massless photons may only occupy longitudinal polarization states $\left(\lambda_{\gamma}= \pm 1\right)[8]$. Thus, the spin configuration of a pure state may be written as

$$
|\gamma\rangle=a_{+}\left|\lambda_{\gamma}=+1\right\rangle+a_{-}\left|\lambda_{\gamma}=-1\right\rangle
$$

Normalization and orthogonality of the basis requires $\left\langle\lambda_{\gamma} \mid \lambda_{\gamma}^{\prime}\right\rangle=\delta_{\lambda_{\gamma} \lambda_{\gamma}^{\prime}}$ and $\left|a_{+}\right|^{2}+\left|a_{-}\right|^{2}=1$. The density matrix is the outer product of this state

$$
\rho(\gamma)=|\gamma\rangle\langle\gamma|=\left(\begin{array}{cc}
\left|a_{+}\right|^{2} & a_{+} a_{-}^{*} \\
a_{-} a_{+}^{*} & \left|a_{-}\right|^{2}
\end{array}\right)
$$

In the specific case of photons with pure linear polarization, the state of the photon may be expressed as

$$
|\gamma\rangle=-\frac{1}{\sqrt{2}}\left(e^{-i \Phi}\left|\lambda_{\gamma}=+1\right\rangle+e^{+i \Phi}\left|\lambda_{\gamma}=-1\right\rangle\right) .
$$

where $\Phi$ is the angle between the photon polarization vector and some reference. In this analysis, the production plane is chosen as the reference. The corresponding density matrix reads

$$
\rho(\gamma)=|\gamma\rangle\langle\gamma|=\frac{1}{2}\left(\begin{array}{cc}
1 & -e^{-2 i \Phi} \\
-e^{2 i \Phi} & 1
\end{array}\right)
$$

The spin-density formalism does not only apply to pure states. Any mixed state photon density matrix can be considered an ensemble of pure states. These may then be decomposed in the space of $2 \times 2$ Hermitian matrices. The identity matrix and the Pauli matrices constitute a complete set in this basis. We may therefore write a general decomposition of the photon spin density matrix as

$$
\rho(\gamma)=\frac{1}{2} I+\frac{1}{2} \mathbf{P}_{\gamma} \cdot \boldsymbol{\sigma}
$$

With the Pauli matrices

$$
\sigma_{x}=\left(\begin{array}{ll}
0 & 1 \\
1 & 0
\end{array}\right), \sigma_{y}=\left(\begin{array}{cc}
0 & -i \\
i & 0
\end{array}\right), \sigma_{z}=\left(\begin{array}{cc}
1 & 0 \\
0 & -1
\end{array}\right) .
$$

When describing partial linear polarization as is the case in the GlueX experiment, $\mathbf{P}_{\gamma}=$ $P_{\gamma}(-\cos 2 \Phi,-\sin 2 \Phi, 0)$, where $P_{\gamma}$ is the degree of polarization of the photon and $\Phi$ is the angle between the photon polarization vector and the production plane of the vector meson. A circularly polarized photon beam may be described by $\mathbf{P}_{\gamma}=P_{\gamma}(0,0, \pm 1)$ with the sign of the $\mathrm{z}$ component corresponding to the helicity of the beam.

\subsubsection{Vector Meson Spin-Density Matrix}

Defining the decomposed components of $\rho(V)$ according to equation 1.2 as $\left(\rho^{0}(V), \rho^{\alpha}(V)\right)=T\left(\frac{1}{2} I, \frac{1}{2} \sigma^{\alpha}\right) T^{\dagger}$ for $\alpha=1,2,3$ and inserting 1.2 into 1.1 allows us to decompose $\rho(V)$ in terms of the helicity amplitudes as

$$
\rho(V)=\rho^{0}+\sum_{i=1}^{3} P_{i} \rho^{i}
$$


where $P_{i}$ is the $i$-th component of $\mathbf{P}_{\gamma}$. The individual components of the spin-density matrix are then explicitly given by

$$
\begin{aligned}
\rho_{\lambda_{V} \lambda_{V}^{\prime}}^{0}(V) & =\frac{1}{2 \mathcal{N}} \sum_{\lambda_{\gamma} \lambda_{N^{\prime}} \lambda_{N}} T_{\lambda_{V} \lambda_{N^{\prime}}, \lambda_{\gamma} \lambda_{N}} T_{\lambda_{V}^{\prime} \lambda_{N^{\prime}}, \lambda_{\gamma} \lambda_{N}}^{*} \\
\rho_{\lambda_{V} \lambda_{V}^{\prime}}^{1}(V) & =\frac{1}{2 \mathcal{N}} \sum_{\lambda_{\gamma} \lambda_{N^{\prime}} \lambda_{N}} T_{\lambda_{V} \lambda_{N^{\prime}},-\lambda_{\gamma} \lambda_{N}} T_{\lambda_{V}^{\prime} \lambda_{N^{\prime}}, \lambda_{\gamma} \lambda_{N}}^{*} \\
\rho_{\lambda_{V} \lambda_{V}^{\prime}}^{2}(V) & =\frac{i}{2 \mathcal{N}} \sum_{\lambda_{\gamma} \lambda_{N^{\prime}} \lambda_{N}} \lambda_{\gamma} T_{\lambda_{V} \lambda_{N^{\prime}},-\lambda_{\gamma} \lambda_{N}} T_{\lambda_{V}^{\prime} \lambda_{N^{\prime}}, \lambda_{\gamma} \lambda_{N}}^{*} \\
\rho_{\lambda_{V} \lambda_{V}^{\prime}}^{3}(V) & =\frac{1}{2 \mathcal{N}} \sum_{\lambda_{\gamma} \lambda_{N^{\prime}} \lambda_{N}} \lambda_{\gamma} T_{\lambda_{V} \lambda_{N^{\prime}}, \lambda_{\gamma} \lambda_{N}} T_{\lambda_{V}^{\prime} \lambda_{N^{\prime}}, \lambda_{\gamma} \lambda_{N}}^{*} .
\end{aligned}
$$

Not all of the helicity amplitudes are independent. Parity conservation leads to the following relation among the amplitudes

$$
T_{-\lambda_{V}-\lambda_{N^{\prime}},-\lambda_{\gamma}-\lambda_{N}}=(-1)^{\left(\lambda_{V}-\lambda_{N^{\prime}}\right)-\left(\lambda_{\gamma}-\lambda_{N}\right)} T_{\lambda_{V} \lambda_{N^{\prime}}, \lambda_{\gamma} \lambda_{N}}
$$

This reduces the number of independent SDMEs by the relations

$$
\begin{aligned}
& \rho_{\lambda \lambda^{\prime}}^{0,1}=(-1)^{\lambda-\lambda^{\prime}} \rho_{-\lambda-\lambda^{\prime}}^{0,1} \\
& \rho_{\lambda \lambda^{\prime}}^{2,3}=-(-1)^{\lambda-\lambda^{\prime}} \rho_{-\lambda-\lambda^{\prime}}^{2,3}
\end{aligned}
$$

Using this parity relation and the hermiticity of the $\rho$ matrices, we find that of the 72 conceivable parameters in our decomposition for the spin density matrix (Four $3 \times 3$ complex valued matrices) only eleven are measurable with polarized beams. With a linearly polarized beam, nine of these parameters may be measured. Three of these are elements from $\rho^{0}\left(\rho_{00}^{0}, \rho_{10}^{0}, \rho_{1-1}^{0}\right)$, four from $\rho^{1}$ $\left(\rho_{00}^{1}, \rho_{10}^{1}, \rho_{11}^{1}, \rho_{1-1}^{1}\right)$, and two from $\rho^{2}\left(\rho_{10}^{2}, \rho_{1-1}^{2}\right)$. Owing to the hermeticity of the matrices, $\rho_{1-1}^{0}$ and $\rho_{1-1}^{1}$ are real and $\rho_{1-1}^{2}$ is purely imaginary. The diagonal elements of each matrix must be real. The $\rho_{10}^{0,1,2,3}$ elements have both real and imaginary components but only the real component is measurable in $\rho_{10}^{0,1}$ and the imaginary component in $\rho_{10}^{2,3}$. This is implied throughout the remainder of this document even when not explicitly noted.

\subsubsection{Angular Distributions}

In the experiment, we reconstruct the $\omega$ meson by measuring its decay products. We derive the angular distribution of these decay products as follows. In the rest frame of the $\omega$ meson, the angular distribution may be written as

$$
\frac{d N}{d \cos \theta d \phi} \equiv W(\cos \theta, \phi)=M \rho(V) M^{\dagger}
$$

where $M$ is the decay amplitude. In this document, the decay angles, $\theta$ and $\phi$, are measured in the helicity system of the $\omega$ meson rest frame. The coordinate system is defined with the z-axis opposite to the recoil proton direction, the y-axis normal to the production plane, and the $\mathrm{x}$-axis chosen to make the system right-handed. In the case of the three-body decay $\omega \rightarrow \pi^{+} \pi^{-} \pi^{0}$ the angles are measured relative to the normal of the decay plane. In the two-body decay $\omega \rightarrow \pi^{0} \gamma$, the angles are measured relative to the radiated photon direction. The choice of quantization axis is arbitrary, but the angles, and therefore the SDMEs, are frame dependent. Other choices commonly used are the Gottfried-Jackson and Adair frames, where the z-axis is chosen in the direction of the incoming photon in the overall center-of-mass (Adair) or vector meson center-of-mass (GJ) frame. Each of 
these different frames can be reached by a rotation around their common y axis. The details of this transformation are described in [9].

For a vector meson decay to pseudoscalars, such as the hadronic decay $\omega \rightarrow \pi^{+} \pi^{-} \pi^{0}$, equation 1.5 may be explicitly written as

$$
W(\cos \theta, \phi)=\sum_{\lambda_{V} \lambda_{V}^{\prime}}\left\langle\theta, \phi|M| \lambda_{V}\right\rangle \rho(V)_{\lambda_{V} \lambda_{V}^{\prime}}\left\langle\lambda_{V}^{\prime}\left|M^{\dagger}\right| \theta, \phi\right\rangle
$$

where $M$ is the decay operator that takes our $\omega$ state of known helicity and decays it to a final state of three pseudoscalars. The decay amplitudes are

$$
\left\langle\theta, \phi|M| \lambda_{V}\right\rangle=C \sqrt{\frac{3}{4 \pi}} D_{\lambda_{V} 0}^{1 *}(\phi, \theta,-\phi) .
$$

where $|C|^{2}$ is related to the decay width of $\omega \rightarrow \pi^{+} \pi^{-} \pi^{0}$. For reference, the Wigner $D$ functions for spin-1 decay are given by

$$
\begin{gathered}
D_{m^{\prime} m}^{j}(\phi, \theta,-\phi)=d_{m^{\prime} m}^{j}(\theta) e^{-i\left(m^{\prime}-m\right) \phi} \\
d_{00}^{1}(\theta)=\cos \theta \quad d_{10}^{1}(\theta)=-\frac{\sin \theta}{\sqrt{2}} \\
d_{11}^{1}(\theta)=\frac{1+\cos \theta}{2} \quad d_{1-1}^{1}(\theta)=\frac{1-\cos \theta}{2} \\
d_{m^{\prime} m}^{j}=(-1)^{m-m^{\prime}} d_{m m^{\prime}}^{j}=d_{-m-m^{\prime}}^{j}
\end{gathered}
$$

By combining equations 1.2, 1.6 and 1.7, and normalizing such that $C=1$ yields the following form for the decay distribution,

$$
W(\cos \theta, \phi)=W^{0}(\cos \theta, \phi)+\sum_{i=1}^{3} \mathbf{P}_{\gamma}^{i} W^{i}(\cos \theta, \phi)
$$

with

$$
\begin{aligned}
W_{h}^{0}\left(\cos \theta, \phi, \rho^{0}\right)= & \frac{3}{4 \pi}\left[\frac{1}{2}\left(1-\rho_{00}^{0}\right)+\frac{1}{2}\left(3 \rho_{00}^{0}-1\right) \cos ^{2} \theta\right. \\
& \left.-\sqrt{2} \operatorname{Re} \rho_{10}^{0} \sin 2 \theta \cos \phi-\rho_{1-1}^{0} \sin ^{2} \theta \cos 2 \phi\right] \\
W_{h}^{1}\left(\cos \theta, \phi, \rho^{1}\right)= & \frac{3}{4 \pi}\left[\rho_{11}^{1} \sin ^{2} \theta+\rho_{00}^{1} \cos ^{2} \theta-\sqrt{2} \operatorname{Re} \rho_{10}^{1} \sin 2 \theta \cos \phi\right. \\
& \left.-\rho_{1-1}^{1} \sin ^{2} \theta \cos 2 \phi\right] \\
W_{h}^{2}\left(\cos \theta, \phi, \rho^{2}\right)= & \frac{3}{4 \pi}\left[\sqrt{2} \operatorname{Im} \rho_{10}^{2} \sin 2 \theta \sin \phi+\operatorname{Im} \rho_{1-1}^{2} \sin ^{2} \theta \sin 2 \phi\right] \\
W_{h}^{3}\left(\cos \theta, \phi, \rho^{3}\right)= & \frac{3}{4 \pi}\left[\sqrt{2} \operatorname{Im} \rho_{10}^{3} \sin 2 \theta \sin \phi+\operatorname{Im} \rho_{1-1}^{3} \sin ^{2} \theta \sin 2 \phi\right] .
\end{aligned}
$$

Here the subscript $h$ indicates the $\omega$ is measured through its hadronic $\omega \rightarrow \pi^{+} \pi^{-} \pi^{0}$ decay. $\rho^{0}$ is of unit trace and $\rho_{11}^{0}=\rho_{-1-1}^{0}$ by parity conservation, so $\rho_{11}^{0}=1 / 2\left(1-\rho_{00}^{0}\right)$. The four terms in equation 1.9 neatly divide the contributions from various incident photon polarizations. $W^{0}$ can be measured with polarized or unpolarized beams, $W^{1}$ and $W^{2}$ require a linearly polarized beam, and $W^{3}$ requires a circularly polarized beam.

In the case of the radiative decay, $\omega \rightarrow \pi^{0} \gamma$, the orientation of the outgoing photon spin must be accounted for in the calculation. The forms of the decay amplitude presented here follow the derivation presented in Reference [10]. There is now an additional sum over the outgoing photon polarizations

$$
W(\cos \theta, \phi)=\sum_{\lambda_{V} \lambda_{V}^{\prime} \Lambda_{\gamma}}\left\langle\Lambda_{\gamma} ; \theta, \phi|M| \lambda_{V}\right\rangle \rho(V)_{\lambda_{V} \lambda_{V}^{\prime}}\left\langle\lambda_{V}^{\prime}\left|M^{\dagger}\right| \theta, \phi ; \Lambda_{\gamma}\right\rangle .
$$


with the decay operator M now acting to decay the $\omega$ meson to $\pi^{0} \gamma$. Depending on the spin of the vector meson, the decay amplitude differs slightly. For transversely polarized vector mesons $\left(\lambda_{V}= \pm 1\right)$ we have in the vector meson rest frame

$$
\left\langle\Lambda_{\gamma} ; \theta, \phi|M| \lambda_{V}= \pm 1\right\rangle=C \sqrt{\frac{3}{8 \pi}}(-1)^{\lambda_{V}} D_{-\lambda_{V} \Lambda_{\gamma}}^{1 *}(\phi, \theta,-\phi) .
$$

While for longitudinally polarized vector mesons $\left(\lambda_{V}=0\right)$ we have

$$
\left\langle\Lambda_{\gamma} ; \theta, \phi|M| \lambda_{V}=0\right\rangle=C \sqrt{\frac{3}{8 \pi}} \Lambda_{\gamma} D_{0 \Lambda_{\gamma}}^{1 *}(\phi, \theta,-\phi) .
$$

where $|C|^{2}$ is related to the decay width of $\omega \rightarrow \pi^{0} \gamma$. Combining equations $1.2,1.6$ and 1.7, and normalizing such that $C=1$ yields an equation of the same form as 1.9 with the following terms:

$$
\begin{aligned}
W_{r}^{0}\left(\cos \theta, \phi, \rho^{0}\right)= & \frac{3}{8 \pi}\left[1-\rho_{11}^{0} \sin ^{2} \theta-\rho_{00}^{0} \cos ^{2} \theta\right. \\
& \left.+\sqrt{2} \operatorname{Re} \rho_{10}^{0} \sin 2 \theta \cos \phi+\rho_{1-1}^{0} \sin ^{2} \theta \cos 2 \phi\right] \\
W_{r}^{1}\left(\cos \theta, \phi, \rho^{1}\right)= & \frac{3}{8 \pi}\left[2 \rho_{11}^{1}+\left(\rho_{00}^{1}-\rho_{11}^{1}\right) \sin ^{2} \theta+\sqrt{2} \operatorname{Re} \rho_{10}^{1} \sin 2 \theta \cos \phi\right. \\
& \left.+\rho_{1-1}^{1} \sin ^{2} \theta \cos 2 \phi\right] \\
W_{r}^{2}\left(\cos \theta, \phi, \rho^{2}\right)= & -\frac{3}{8 \pi}\left[\sqrt{2} \operatorname{Im} \rho_{10}^{2} \sin 2 \theta \sin \phi+\operatorname{Im} \rho_{1-1}^{2} \sin ^{2} \theta \sin 2 \phi\right] \\
W_{r}^{3}\left(\cos \theta, \phi, \rho^{3}\right)= & -\frac{3}{8 \pi}\left[\sqrt{2} \operatorname{Im} \rho_{10}^{3} \sin 2 \theta \sin \phi+\operatorname{Im} \rho_{1-1}^{3} \sin ^{2} \theta \sin 2 \phi\right] .
\end{aligned}
$$

Here the subscript $r$ indicates we are measuring the angular distribution in the radiative $\omega \rightarrow$ $\pi^{0} \gamma$ decay. Note that this result differs in sign from that presented in Reference [10]. We believe this form is correct and produces fit results consistent with the hadronic decay of $\omega$ (see Chapter 5 ).

\subsection{The Oh, Titov, Lee Model}

At GlueX energies, the dominant contributions to $\omega$ production are t-channel Pomeron and t-channel pseudoscalar exchange. Here we adapt a model developed by Oh, Titov and Lee (hereafter referred to as OTL) in Reference [11] to investigate the predictions for the SDMEs based on these exchange mechanisms. The model presented was initially developed in order to investigate contributions to high angle scattering by potential $N^{*}$ states, but the amplitudes developed to describe the nonresonant Pomeron and pseudoscalar exchanges are applicable to this work.

The relevant amplitudes contributing to the forward production of $\omega$ in the model may be written as

$$
I_{t o t}=I_{\mathbb{P}}+I_{\pi}+I_{\eta}
$$

where $I_{\mathbb{P}}, I_{\pi}$ and $I_{\eta}$ refer to Pomeron, pion and eta exchange contributions in the t-channel. The Pomeron exchange amplitude shown schematically in Figure 1.6a, is described by the DonnachieLandshoff model [12]

$$
I_{\mathbb{P}}=i M_{0(s, t)} \bar{u}_{m_{f}}\left(p^{\prime}\right) \varepsilon_{\mu}^{*}(\omega)\left\{\not k g^{\mu \nu}-k^{\mu} \gamma^{\nu}\right\} \varepsilon_{\nu}(\gamma) u_{m_{i}}(p)
$$

with

$$
M_{0}(s, t)=C_{V} F_{1}(t) F_{V}(t)\left(\frac{s}{s_{0}}\right)^{\alpha_{\mathbb{P}}(t)-1} \exp \left\{-\frac{i \pi}{2}\left[\alpha_{\mathbb{P}}(t)-1\right]\right\},
$$




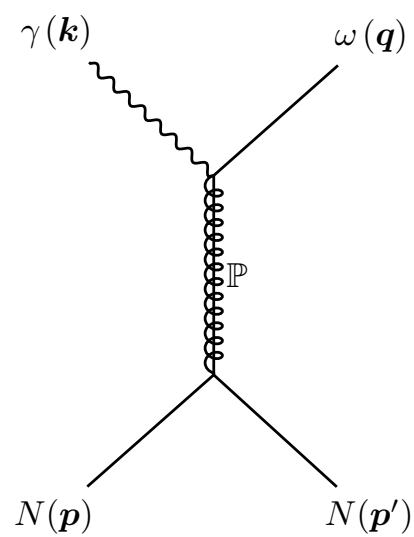

(a)

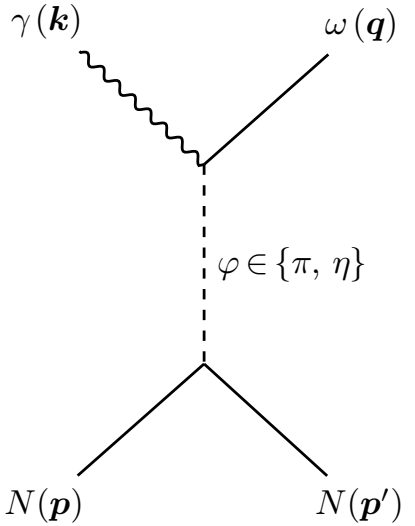

(b)

Figure 1.6: Feynman diagrams representing the two forward processes included in the OTL model, (a) t-channel Pomeron exchange and (b) t-channel pseudoscalar exchange.

$$
F_{1}(t)=\frac{4 M_{N}^{2}-2.8 t}{\left(4 M_{N}^{2}-t\right)\left(1-t / t_{0}\right)^{2}} \text { and } F_{V}(t)=\frac{1}{1-t / M_{V}^{2}} \frac{2 \mu_{0}^{2}}{2 \mu_{0}^{2}+M_{V}^{2}-t} .
$$

The Regge trajectory for the Pomeron is well-measured and given by $\alpha_{\mathbb{P}}(t)=1.08+0.25 t$. The coupling strength is $C_{V}=12 \sqrt{4 \pi \alpha_{e m}} \beta_{0}^{2} / f_{v}$ where $f_{V}=17.05$ is the vector meson decay constant. The remaining free parameters of the model were fit to existing total cross section measurements for $\omega, \rho$ and $\phi$ photoproduction, yielding $\mu_{0}^{2}=1.1 \mathrm{GeV}^{2}, \beta_{0}=2.05 \mathrm{GeV}^{-1}$, and $s_{0}=4.0 \mathrm{GeV}^{2}$.

The t-channel pseudoscalar meson exchange $(\varphi)$ is described by effective Lagrangians characterizing the $\omega \gamma \varphi$ and $\varphi N N$ vertices,

$$
\begin{aligned}
\mathcal{L}_{\omega \gamma \varphi} & =\frac{e g_{\omega \gamma \varphi}}{M_{V}} \epsilon^{\mu \nu \alpha \beta} \partial_{\mu} \omega_{\nu} \partial_{\alpha} A_{\beta} \varphi \\
\mathcal{L}_{\varphi N N} & =-i g_{\pi N N} \bar{N} \gamma_{5} \tau_{3} N \pi^{0}-i g_{\eta N N} \bar{N} \gamma_{5} N \eta .
\end{aligned}
$$

These predict the amplitude for $\varphi \in\{\pi, \eta\}$ in Figure 1.6b as

$$
I_{\varphi}=-\frac{i F_{\varphi N N}(t) F_{\omega \gamma \varphi}(t)}{t-M_{\varphi}^{2}} \frac{e g_{\omega \gamma \varphi} g_{\varphi N N}}{M_{V}} \bar{u}_{m_{f}}\left(p^{\prime}\right) \gamma_{5} u_{m_{i}}(p) \epsilon^{\mu \nu \alpha \beta} q_{\mu} k_{\alpha} \varepsilon_{\nu}^{*}(\omega) \varepsilon_{\beta}(\gamma) .
$$

The form factors are assumed to take the form

$$
F_{\varphi N N}(t)=\frac{\Lambda_{\varphi}^{2}-M_{\varphi}^{2}}{\Lambda_{\varphi}^{2}-t} \text { and } F_{\omega \gamma \varphi}(t)=\frac{\Lambda_{\omega \gamma \varphi}^{2}-M_{\varphi}^{2}}{\Lambda_{\omega \gamma \varphi}^{2}-t}
$$

Here the cutoff parameters for the $\pi$ exchange are taken to be $\Lambda_{\pi}=0.6 \mathrm{GeV}$ and $\Lambda_{\omega \gamma \pi}=0.7 \mathrm{GeV}$. These are determined by a fit to existing forward-cross-section data. The cutoff parameters for the $\eta$ exchange are taken to be $\Lambda_{\eta}=1.0 \mathrm{GeV}$ and $\Lambda_{\omega \gamma \eta}=0.9 \mathrm{GeV}$ and were determined by a study of $\phi$ photoproduction [13]. The contribution of $\eta$-exchange to the model is very small, so the particular choice of these parameters has little impact on the results.

This model has been implemented in $\mathrm{C}++$ using the $\mathrm{qft}++$ package for numerical object-oriented quantum field theory calculations [14]. Equations 1.4a-1.4c are then used to project the SDMEs from the OTL amplitudes. The model predicts the relative contributions of the two exchange mechanisms. While the cross section is not measured as part of this thesis, the predictions based on the model are shown in Figure 1.7. At very high energies, the production is dominated by Pomeron exchange, 


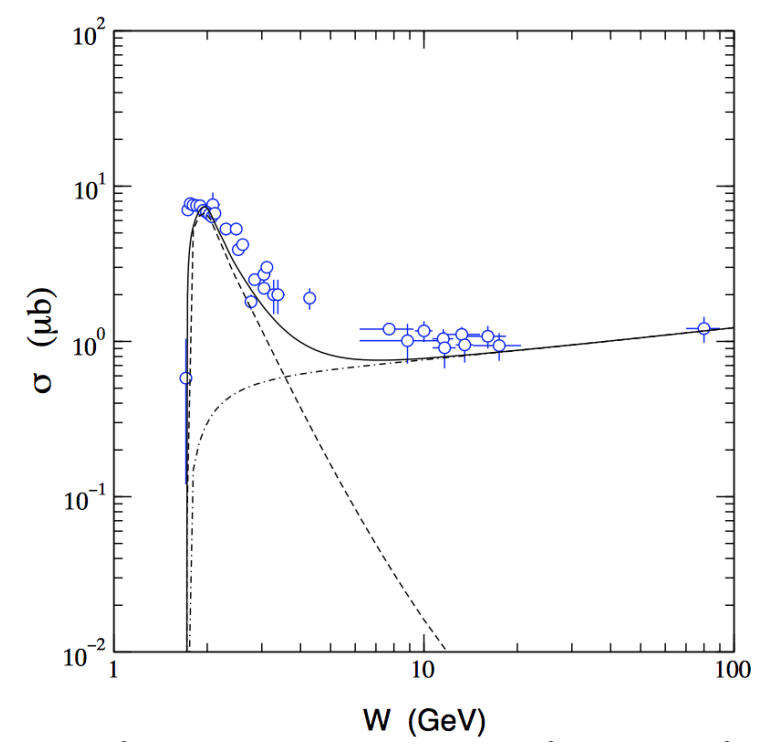

Figure 1.7: Existing $\omega$ photoproduction cross section measurements as a function of center-of-mass energy. The solid line is the full calculation of the OTL model. The dashed line indicates contributions from pseudoscalar exchange, while dot-dashed line indicates the contribution from Pomeron exchange. Reproduced from [11].

while at lower energies pseudoscalar exchange dominates. At GlueX energy, $E_{\gamma}=8.4-9.0 \mathrm{GeV}$ $(W=4.08-4.21 \mathrm{GeV})$, Pomeron exchange is the dominant contribution but pseudoscalar exchange is not completely suppressed, making up roughly $30 \%$ of th total cross section. Therefore, our SDMEs are sensitive to contributions from both exchange mechanisms. The predictions of the model for the SDMEs measured in this thesis are shown in Figure 1.8. Comparisons between this simple phenomenological model and the features seen in the fits to our data are discussed in Section 5.5 .

In this thesis, ongoing measurements of the spin-density matrix elements (SDMEs) in $\omega$ photoproduction using some of the first data from the GlueX experiment are described. These measurements are unique in that they are the first to be exclusively measured simultaneously in the decays $\omega \rightarrow \pi^{+} \pi^{-} \pi^{0}$ and $\omega \rightarrow \pi^{0} \gamma$ owing to the unique detection capability of the GlueX detector. In the energy region of the GlueX experiment, there has only been one previous measurement of $\omega$ photoproduction made at SLAC in the early 1970s [15]. This experiment used a beam of 9.3 $\mathrm{GeV}$ linearly polarized photons produced via Compton backscattering with a linear polarization fraction of $0.77 \pm 0.02$. The differential cross sections were measured, as were the SDMEs in the $\pi^{+} \pi^{-} \pi^{0}$ decay of the $\omega$ using 1,195 selected events. The data presented in this thesis represent a two order-of-magnitude improvement in statistics over this measurement. Our measurements of the SDMEs are compared with the SLAC results in Chapter 5. In addition to providing a check of the GlueX reconstruction performance, the results of this thesis can be used to help constrain models of $\omega$ photoproduction in the medium energy regime that may be applicable in interpretation of hybrid meson decays as analysis of the experimental data progresses. 

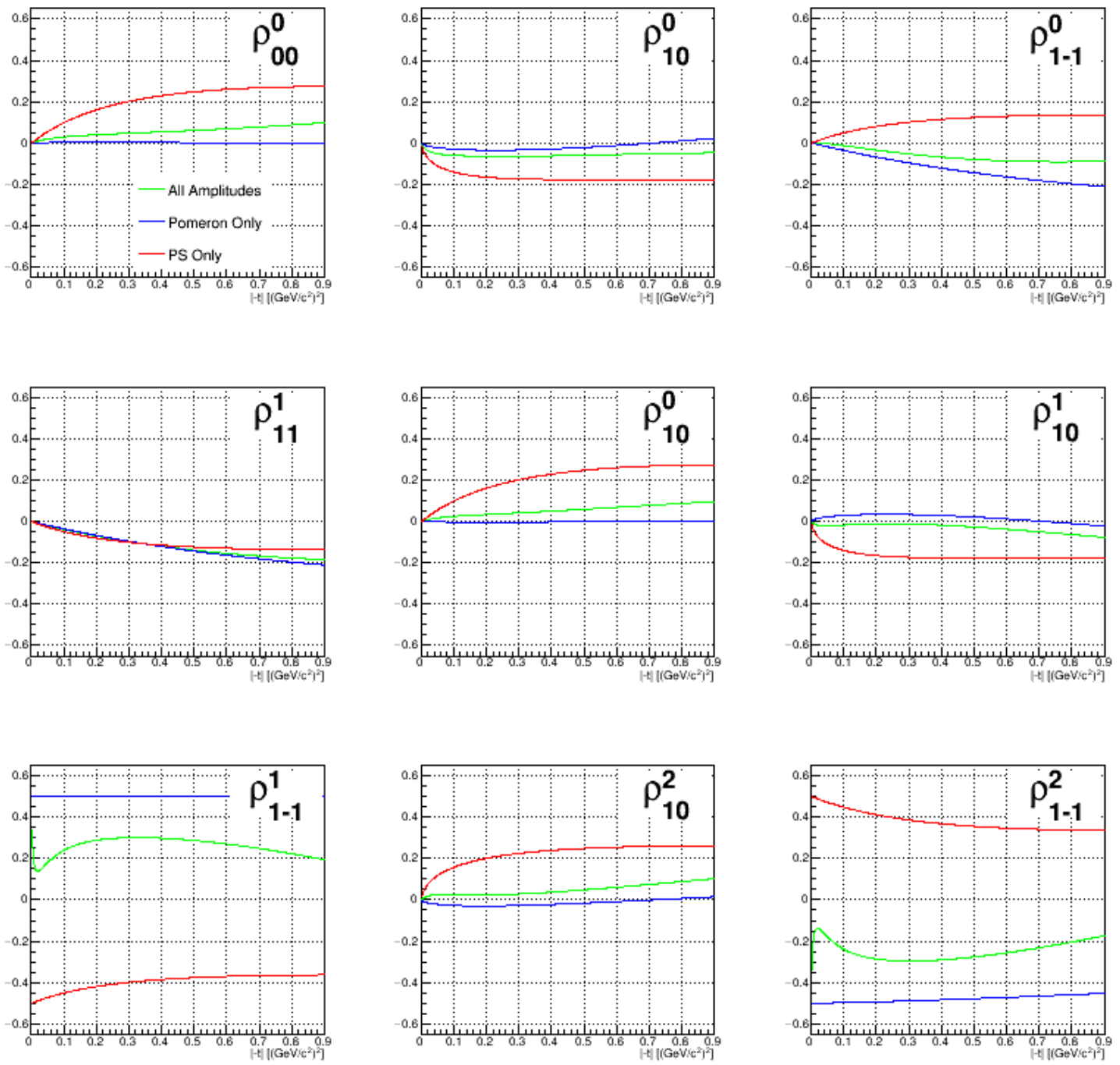

Figure 1.8: Predictions for the nine measurable SDMEs in $\omega$ photoproduction with a linear polarized beam in the $\omega$ helicity frame from the OTL model. The blue line indicates contributions from Pomeron exchange, the red line indicates the contribution from pseudoscalar exchange, and the green line indicates the full model combining the two exchange mechanisms. 


\section{Chapter 2}

\section{The GlueX Detector}

GlueX is the first experiment to run in Hall D of the Continuous Electron Beam Accelerator Facility (CEBAF) located at Jefferson Lab in Newport News, Virginia. The strength of the GlueX detector is the capability to detect both charged and neutral particles with robust particle identification. This capability coupled with a tagged photon beam allow for exclusive reconstruction with nearly hermetic coverage, which is ideal for partial wave analyses that will be used to explore the hybrid meson spectrum. The GlueX detector can be roughly divided into four main components that are described in this chapter: beamline instrumentation, calorimetry, particle identification, and charged particle tracking. A picture of the completed main spectrometer of the GlueX detector can be found in Figure 2.1, and a schematic overview of the detector elements can be found in Figure 2.2.

\subsection{Photon Beamline}

The GlueX detector in Hall D was constructed as part of the $12 \mathrm{GeV}$ upgrade project that doubled the maximum energy of CEBAF. An overview of the $12 \mathrm{GeV}$ upgrade project is shown in Figure 2.3. CEBAF is capable of parallel operations of up to four experimental halls. A detailed schematic of many of the critical beamline components can be found in Figure 2.4. The beam to halls A,B, and $\mathrm{C}$ may be extracted after $1-5$ passes through the accelerator. An additional half-pass through the machine extracts a $250 \mathrm{MHz}$ electron beam to Hall D. An overview of the layout of the photon beamline showing the relative location of the tagger hall, collimator cave, and experimental hall is shown in Figure 2.5.

Once it has reached the tagger hall, the electron beam passes through a $50 \mu \mathrm{m}$ diamond radiator. Details of the diamond radiator fabrication and performance can be found in Reference [17]. By aligning the electron beam with the specific crystal lattice of the diamond, the bremsstrahlung photons emitted can be made to be partially linearly polarized [18]. The direction of the linear polarization can be controlled by rotating the crystal. We refer to linear polarization parallel to the floor as PARA and perpendicular to the floor PERP. This coherent component is predominantly along the beam direction at small angles $(<25 \mu \mathrm{rad})$ while the incoherent bremsstrahlung radiation has a broader angular distribution. The energy at which the coherent radiation peaks is also related to the orientation of the crystal relative to the electron beam. The produced photon beam then travels from the tagger hall into the collimator cave. There the beam passes through a $3.4 \mathrm{~mm}$ or $5.0 \mathrm{~mm}$ diameter tungsten collimator in order to select photons with small angles relative to the electron beam. This enhances the coherent component of the beam entering the experimental hall as illustrated in Figure 2.6. The important goals of the beamline instrumentation are to measure the energy, polarization and flux of the incoming photon beam. This is achieved using three detector systems: the photon tagger, triplet polarimeter, and pair spectrometer. 


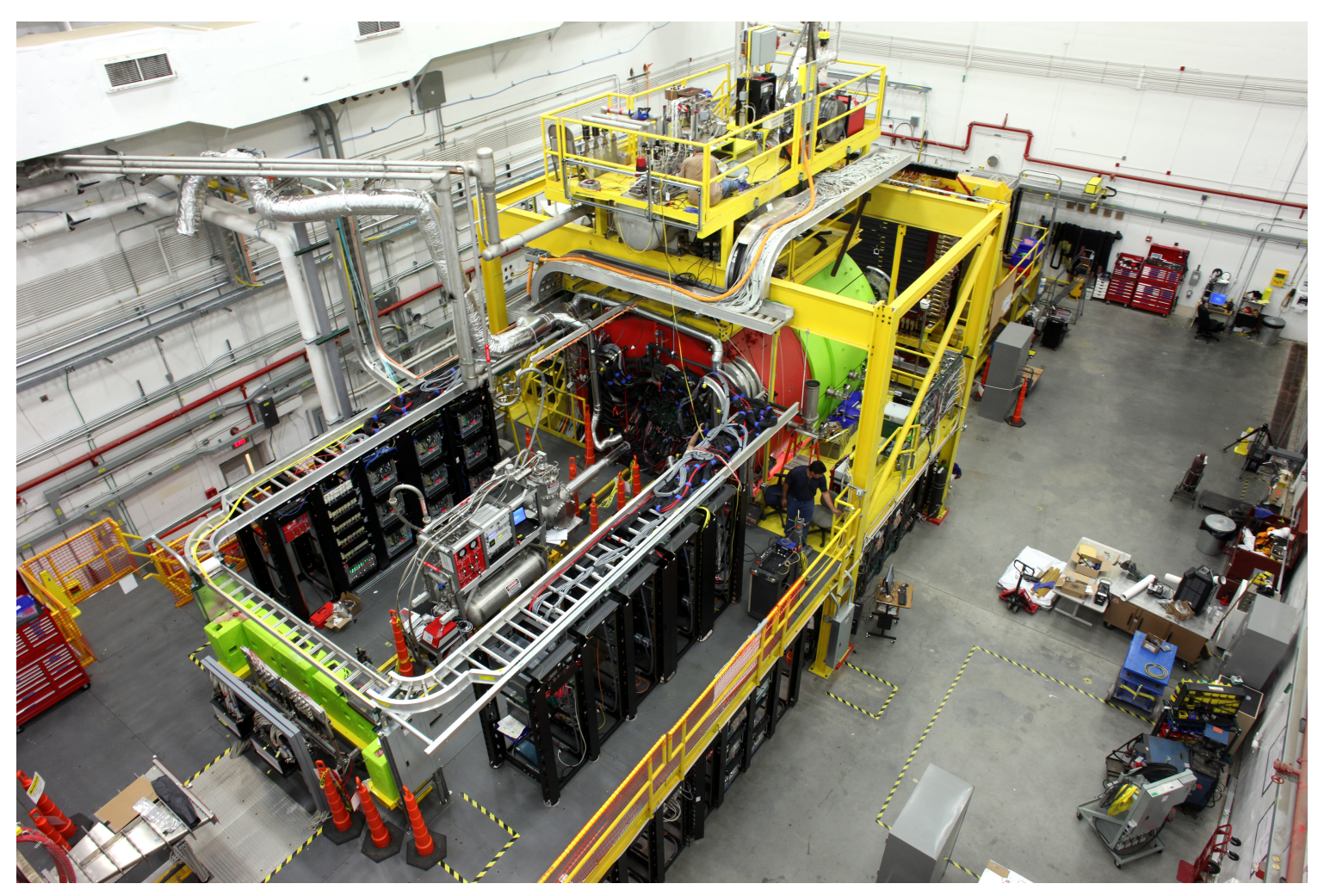

Figure 2.1: Picture of the completed GlueX spectrometer taken in August, 2014.

\subsubsection{Photon Tagger}

Most of the beam electrons incident on the diamond radiator pass through without interacting and are bent by the $1.5 \mathrm{~T}$ dipole tagger magnet to the electron beam dump. Electrons that do interact in the radiator and have lost more than $25 \%$ of their energy are deflected into an array of scintillator counters that comprise the tagging system. The tagging system is broken into two detectors. The tagger microscope (TAGM) covers the range of energy from 8.1-9.1 GeV with energy resolution of $0.1 \%$ and is designed to measure the coherent peak with high resolution. The tagger hodoscope (TAGH) covers the rest of the range from 3-12 GeV. This detector is excluded from the analysis in this thesis since polarization in this energy region is small. A picture of the tagger array is found in Figure 2.7. The timing resolution of the TAGM measured relative to the accelerator RF signal during the Spring 2016 run period is shown in Figure 2.8. For more details on construction and performance of the TAGM, see [19].

\subsubsection{Triplet Polarimeter}

An important experimental parameter is the polarization fraction of the photon beam. One method of determining this value is by measuring "triplet photoproduction" on a nuclear target. Triplet photoproduction is related to the more common $e^{+} e^{-}$pair production off the nucleus of the atom. In pair production we have $\gamma Z \rightarrow Z e^{+} e^{-}$. In the semi-classical approximation the massive nucleus $Z$ remains approximately at rest, and the $e^{+} e^{-}$pair is produced in the direction of the incoming photon. Triplet production differs in that the interaction of the photon occurs with one of the electrons of the atom. We then have $\gamma e^{-} \rightarrow e^{-} e^{+} e^{-}$. Our approximation of the heavy target in pair production no longer holds and the recoil electron may be detected. The angular distribution 


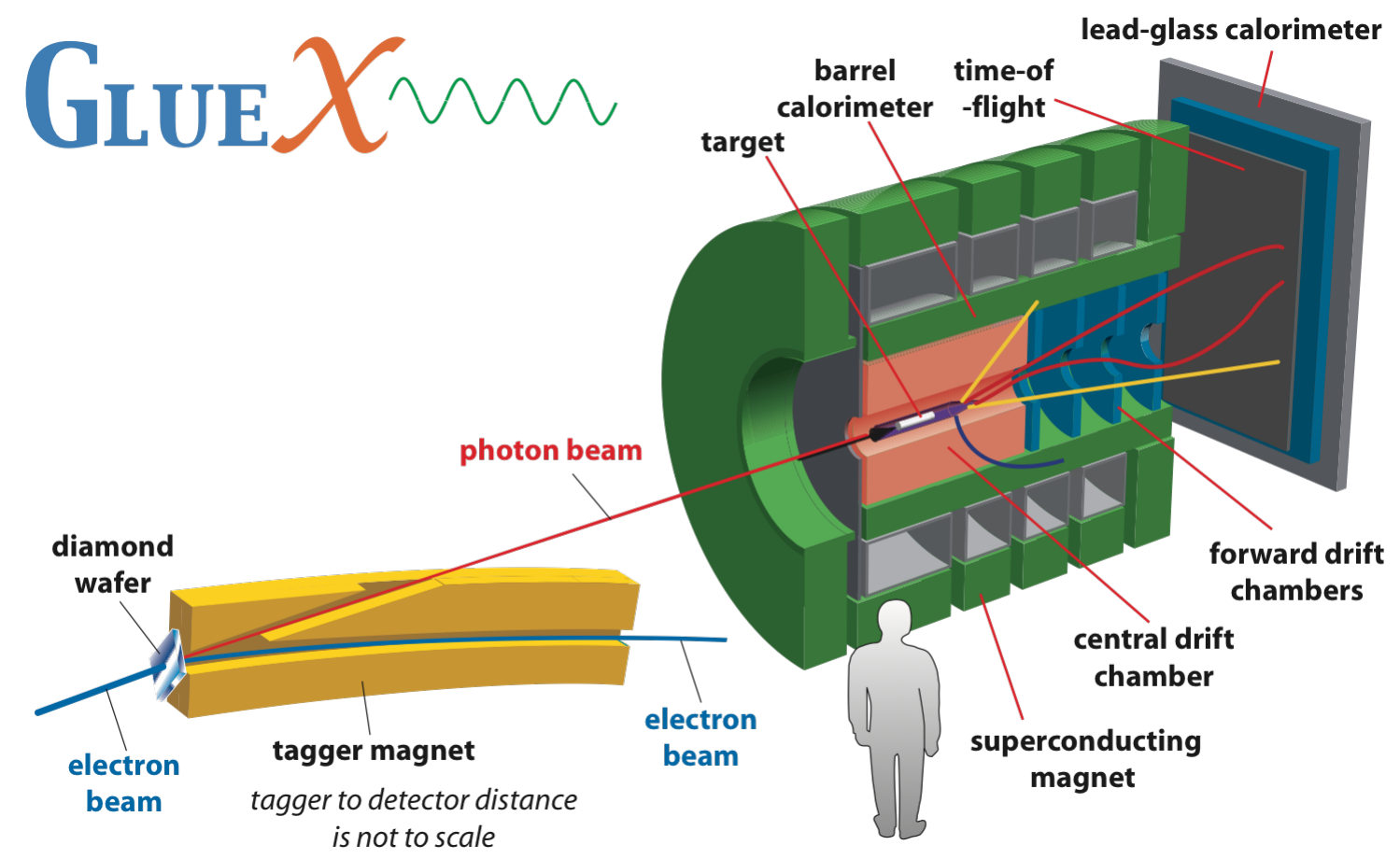

Figure 2.2: Illustration of the GlueX experiment.

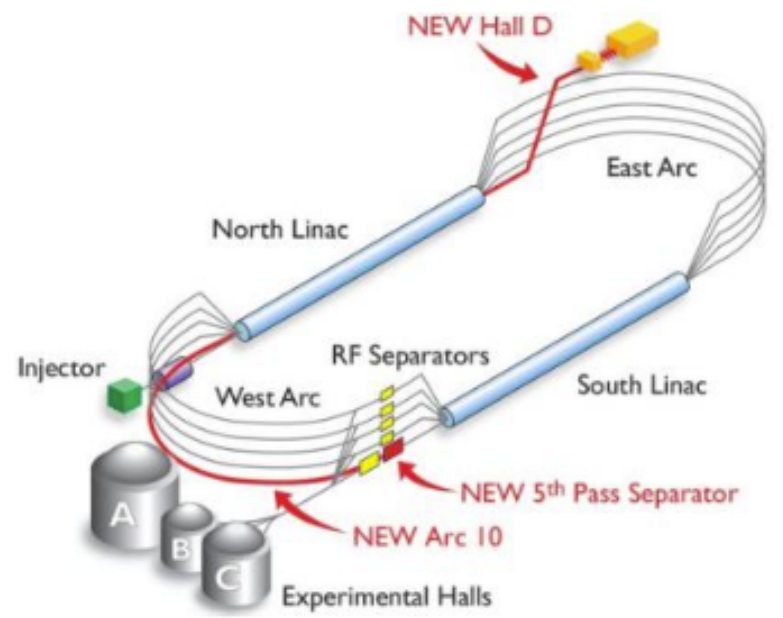

Figure 2.3: Overview of $12 \mathrm{GeV}$ upgrade project of the CEBAF accelerator (not to scale).

of the recoil electron encodes information about the polarization of the incident photon. The cross section for triplet photoproduction is given as

$$
\sigma_{t}(\phi)=\sigma_{0}[1-P \Sigma \cos (2 \phi)]
$$

where $\sigma_{0}$ is the unpolarized cross section, $P$ is the degree of polarization of the photon beam, $\phi$ is the azimuthal angle of the recoil electron with respect to the polarization plane of the photon, and 


\section{JEFFERSON LAB 12 GeV BEAMLINE}

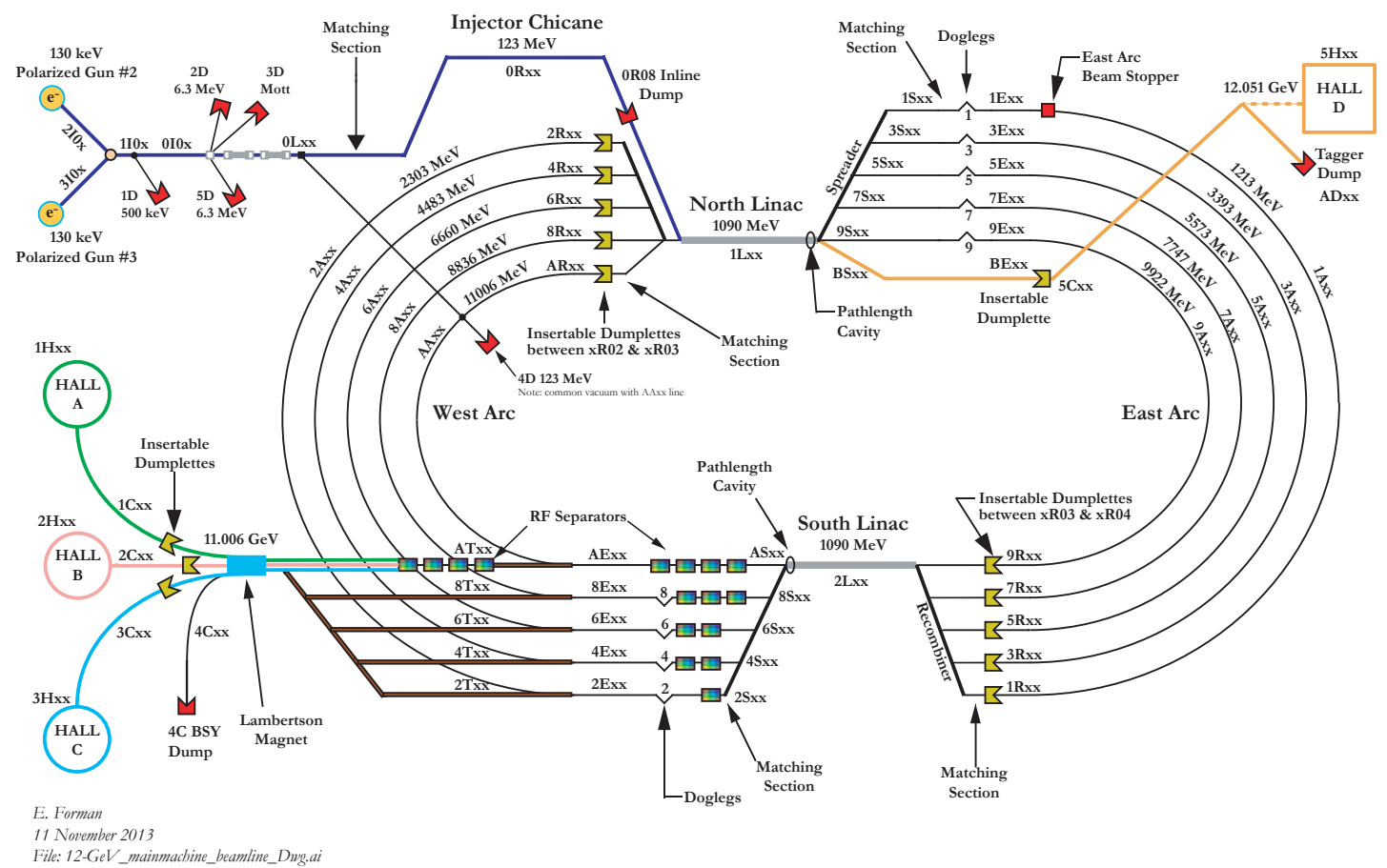

Figure 2.4: Detailed illustration of beamline components of the CEBAF accelerator (not to scale). Reproduced from [16].

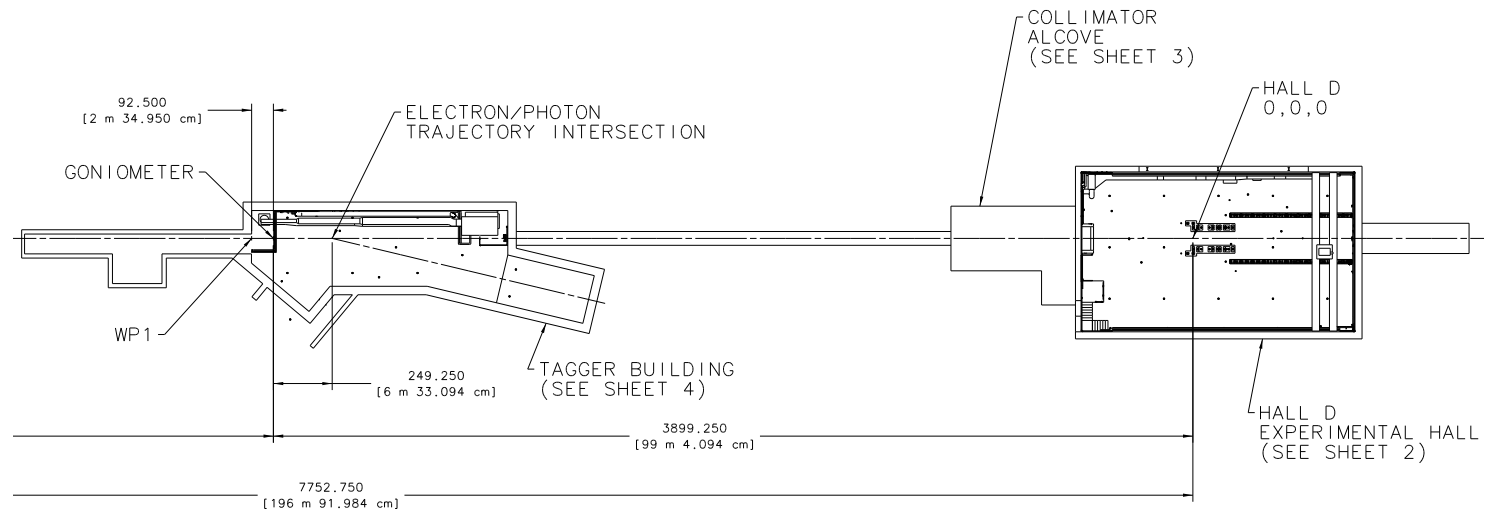

Figure 2.5: Schematic of the Hall D complex including the experimental hall and photon tagging facility. JLab Drawing number D000000000-4002_RevB.

$\Sigma$ is the beam asymmetry for triplet photoproduction [20]. This is a QED process and therefore $\Sigma$ can be calculated through perturbative methods and is determined to be about 0.2. For details of 


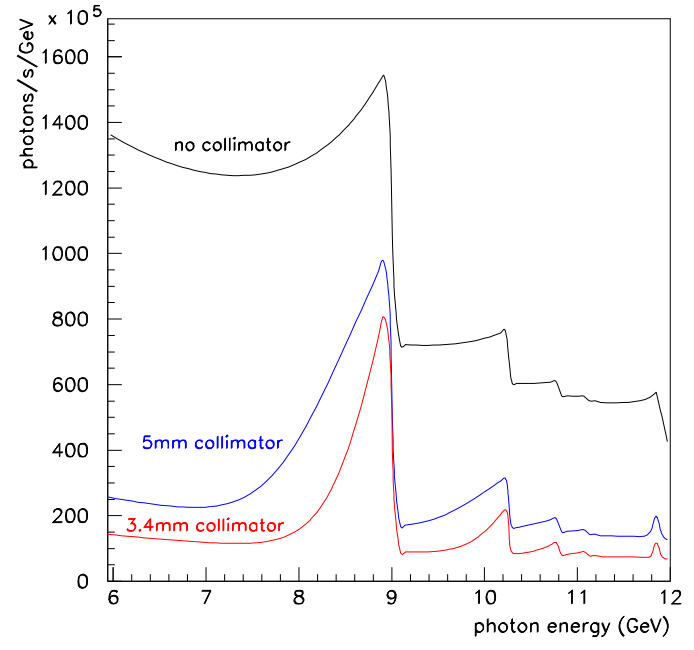

(a) Photon Flux Vs. $E_{\gamma}$

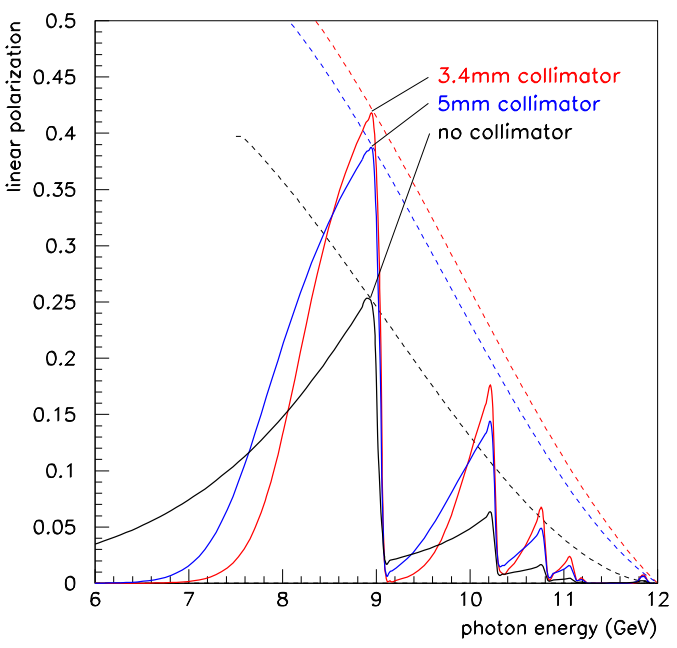

(b) Linear polarization fraction Vs. $E_{\gamma}$

Figure 2.6: Plots showing the effect of collimation on (a) the beam profile, and (b) the polarization fraction. These are simulated for a $15 \mu \mathrm{m}$ thick diamond radiator with a $1 \mu \mathrm{A}$ electron beam current.

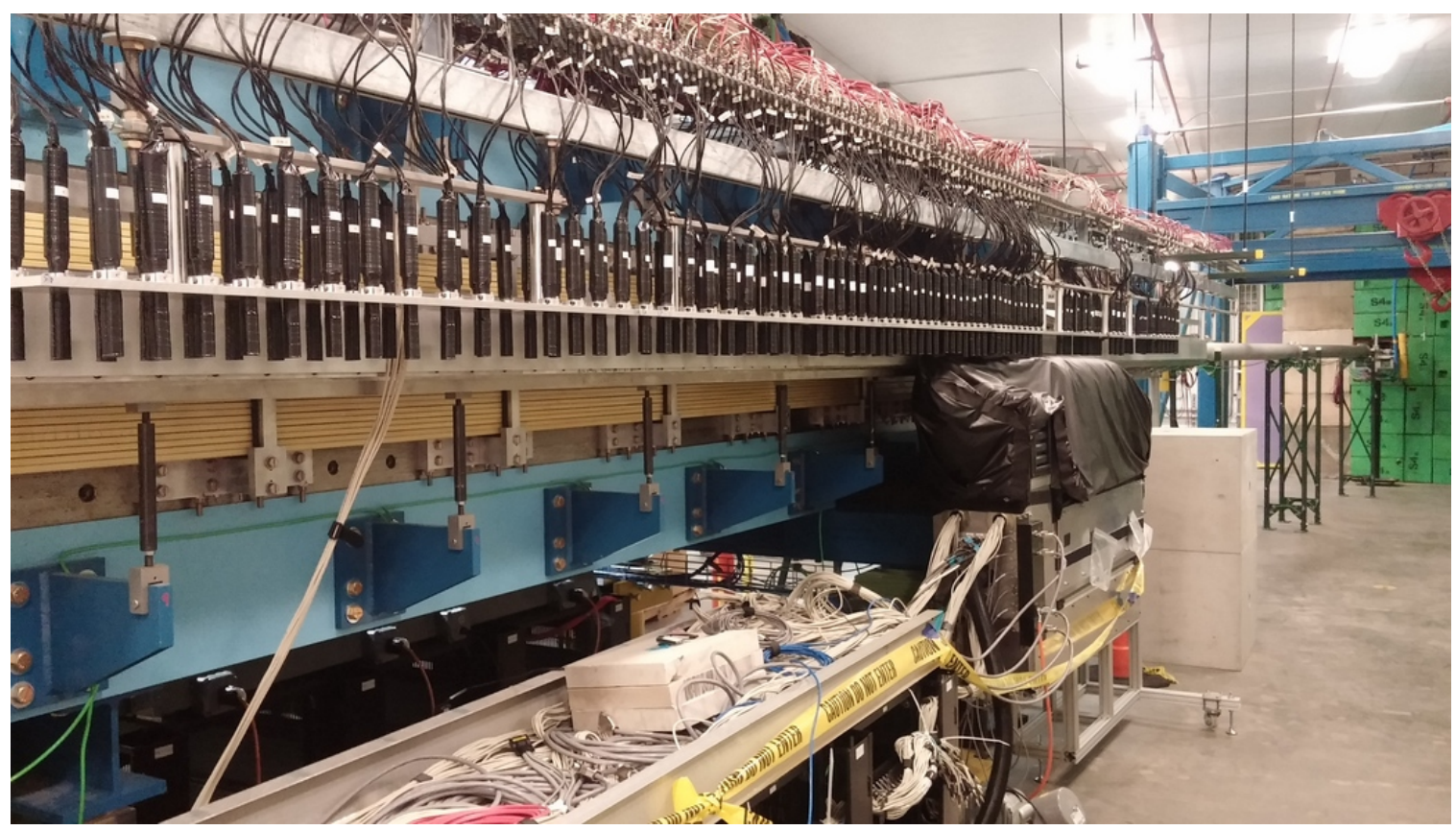

Figure 2.7: Picture of the tagger detectors. The TAGM is located under the black light-tight shroud near the center of the image. The beam dump is to the right.

this calculation, see [20]. In order to extract our beam polarization, the task is to measure the cross section as a function of $\phi$.

The Triplet Polarimeter (TPOL) is designed for just such a measurement. The triplet polarimeter 


\section{Timing resolution $(\sigma)$}

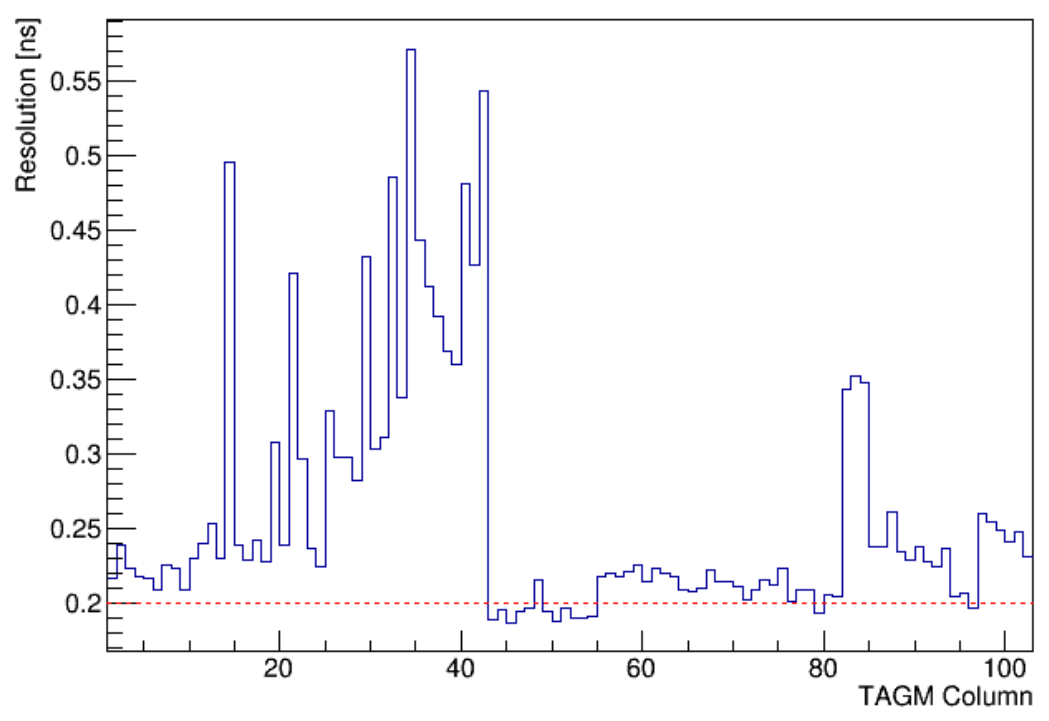

Figure 2.8: TAGM timing resolution for each of the readout channels. The dashed red line is the design resolution. Many of the installed fibers did not reach the design specification for the Spring 2016 run. They have since been replaced.

is placed in the photon beamline downstream of the collimator, before the pair spectrometer. The entire device is operated under vacuum. A $75 \mu \mathrm{m}$ beryllium foil is inserted into the photon beamline before a flat doughnut-shaped silicon strip detector (SSD). The SSD is broken into 32 azimuthal segments and measures the angle and energy of the recoil electron. This measurement is performed in tandem with the main experiment and is triggered by the coincident $e^{+} e^{-}$pair in the Pair Spectrometer. An image of the interior of the TPOL vacuum enclosure is shown in Figure 2.9. The measured azimuthal distributions of the electrons for two polarization directions in the Spring 2016 run period at $E_{\gamma}=8.95 \mathrm{GeV}$ are shown in Figure 2.10. The polarization fractions as a function of energy extracted from fits to these distributions are shown in Figure 2.11.

The results for the polarization fraction presented here are used in the extraction of the spin density matrix elements in Chapter 5 . The average polarization for accepted events in $\gamma p \rightarrow p \pi^{+} \pi^{-} \pi^{0}$ are found in Table 2.1. While the TPOL provides our best independent measurement of the beam polarization, there are additional measurements that we can use to cross-check this result. Comparisons to asymmetries in $\rho$ and $\pi^{0}$ production can be found in [21]. The results from the TPOL are consistent with the $\pi^{0}$ beam asymmetry, and also consistent with results in exclusive $\rho$ production. Measurements based on fitting the energy spectrum of the photon beam by the method in [22] also yield consistent results with these measurements. The TPOL measurement should, in principle, be the cleanest method for extracting the beam polarization, hence these results are used in the analysis presented in this thesis (for more discussion see Chapter 5). These values differ from those presented in Reference [23] due to a systematic in the determination of the polarization that was discovered after publication. The measurements presented in the reference are still consistent with these new values to within the claimed systematic uncertainties.

\subsubsection{Pair Spectrometer}

The Pair Spectrometer (PS) is the last beamline component before the beam enters the main spectrometer. The purpose of the PS is to measure the flux of the incoming photon beam as a function 


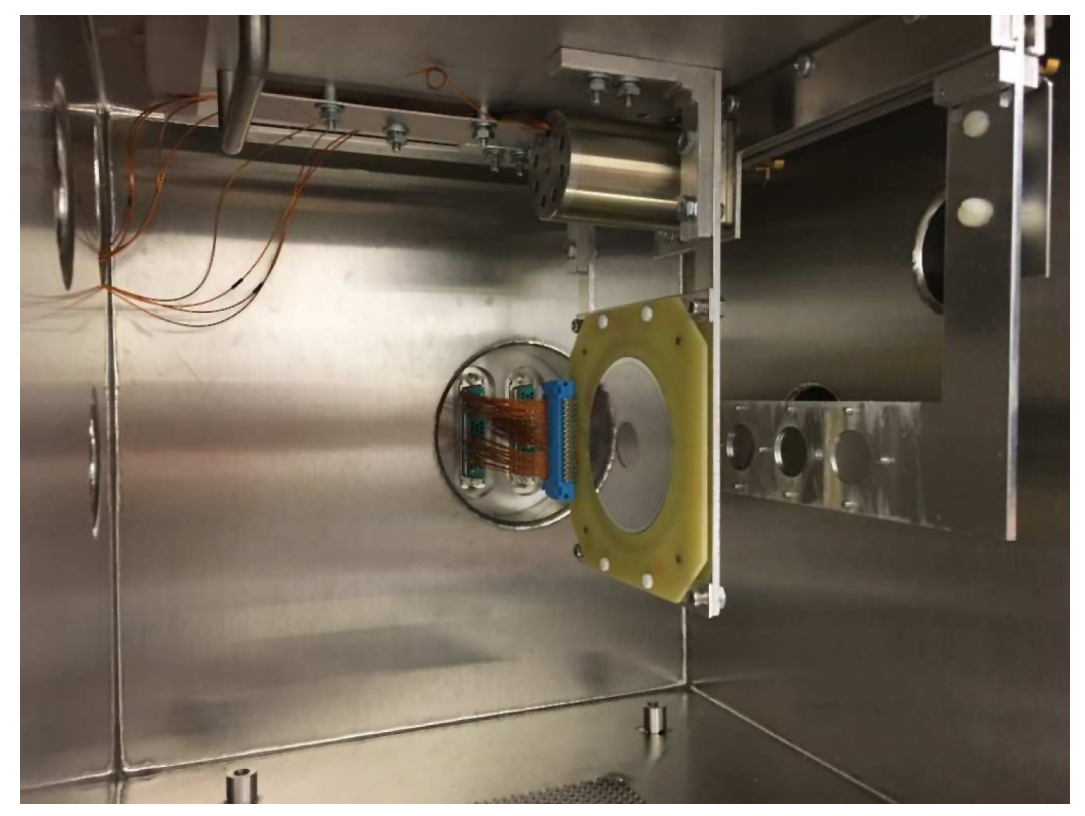

Figure 2.9: Picture of the TPOL detector inside the vacuum enclosure. The beam enters from the right, passes through the beryllium foil mounted on the L-shaped beam, and proceeds into the experimental hall. The SSD is mounted after the beryllium foil. Reproduced from [20].

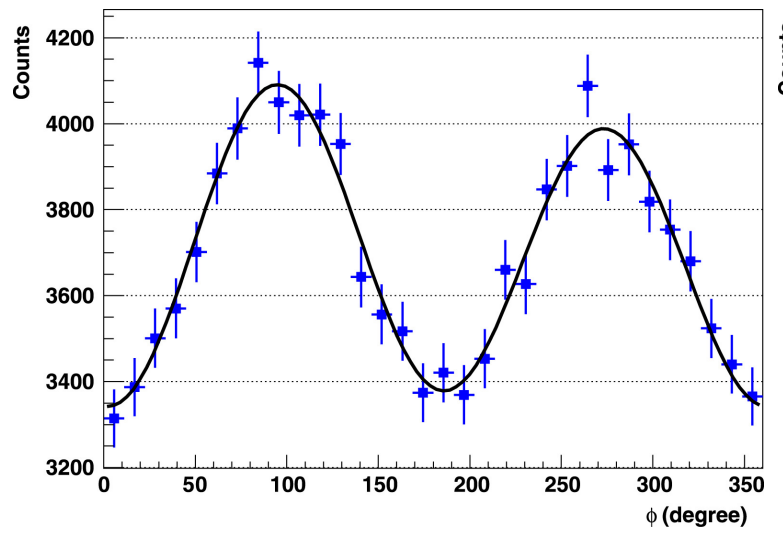

(a) PARA orientation

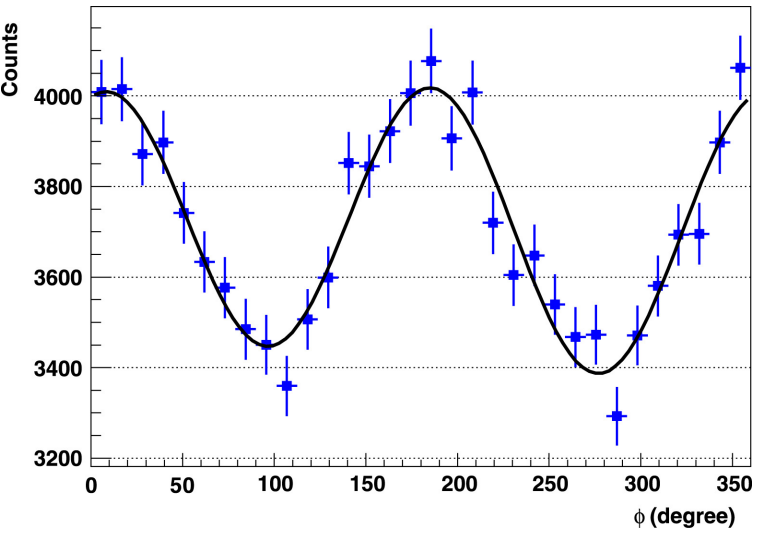

(b) PERP orientation

Figure 2.10: Measured TPOL angular distribution at $E_{\gamma}=8.95 \mathrm{GeV}$. The two plots are for (a) photon polarization direction parallel to the floor (PARA) and (b) photon polarization direction perpendicular to the floor (PERP). Here the angle $\phi$ is measured relative to the same reference direction in both (a) and (b) so that the $\frac{\pi}{2}$ shift in the polarization direction is evident. Reproduced from $[20]$.

of energy and to serve as a trigger for the TPOL measurement. This is achieved by measuring $e^{+} e^{-}$pairs produced off the TPOL radiator. The detector consists of a large dipole magnet that deflects the two particles in opposite directions based on their charge. These are then detected in a set of scintillating detectors that determine the energy of each particle by measuring the amount of deflection experienced in the dipole. The energy spectrum determined by the PS for one run in the Spring 2016 data set is shown in Figure 2.12. More information can be found in [24]. 


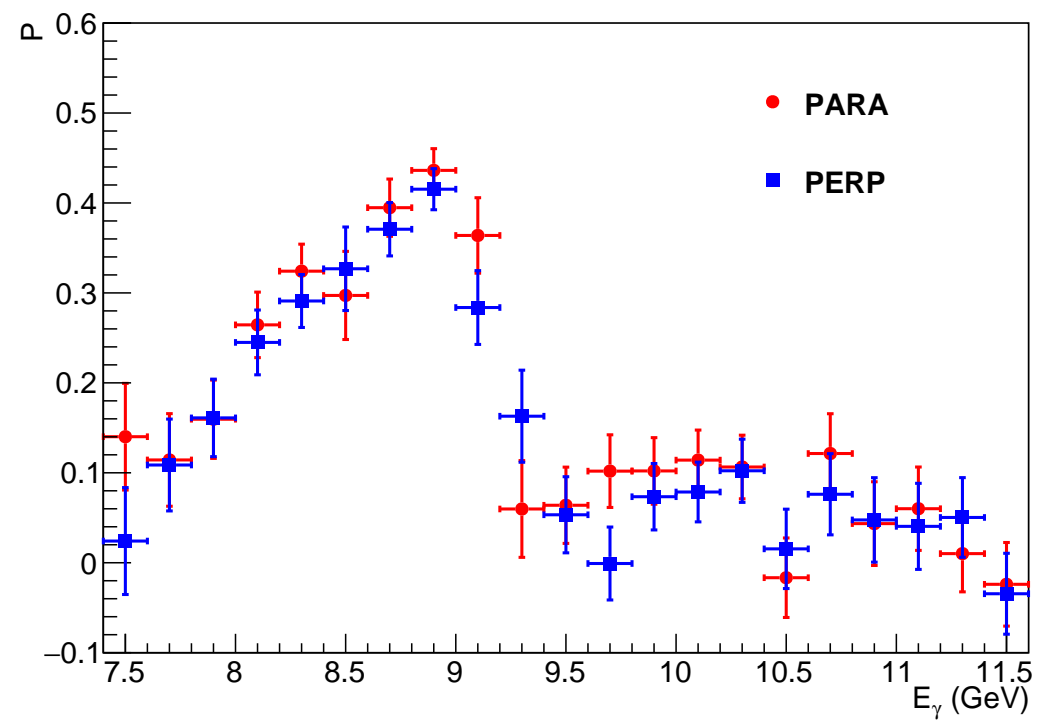

Figure 2.11: Polarization fraction as a function of beam energy for the Spring 2016 run period. Reproduced from [20].

\begin{tabular}{|c|c|c|c|}
\hline Polarization Direction & Polarization Fraction & $\sigma_{\text {pol }}^{\text {stat. }}$ & $\sigma_{\text {pol }}^{\text {syst. }}$ \\
\hline PARA & 0.387 & 0.008 & 0.006 \\
PERP & 0.378 & 0.008 & 0.006 \\
\hline
\end{tabular}

Table 2.1: Energy weighted polarization fraction for PARA and PERP polarization orientations in the Spring 2016 run period. Systematic errors are assigned as in Reference [23].

\subsection{Liquid Hydrogen Target}

Once the photon beam reaches the main spectrometer, it is incident on a liquid hydrogen target. The target cell is $30 \mathrm{~cm}$ long and conically shaped. It is tapered from upstream to downstream, with the diameter decreasing from 2.42 to $1.56 \mathrm{~cm}$. The target nominally operates around $18^{\circ} \mathrm{K}$ and 18 psia. It is surrounded by a Rohacell layer, followed by the Start Counter. This assembly is inserted into the bore of the Central Drift Chamber. An image of the target cell without the Rohacell layer is found in Figure 2.13. More details on the target specifications may be found in [16].

\subsection{Calorimetry}

We have now progressed to the details of the main spectrometer. A top-down schematic of the subsystems that comprise the baseline GlueX detector is shown in Figure 2.14. The calorimeters are the final detectors on the path of particles coming from the target. They are designed for the detection of final-state photons present in the majority of channels targeted for hybrid meson searches in the experiment. They have also served as a useful filter for leptonic final states through measurement of $\mathrm{E} / \mathrm{p}$. The Barrel Calorimeter (BCAL) is the outermost detector in the bore of the GlueX solenoid covering polar angles from $11^{\circ}$ to $126^{\circ}$. Particles missing the downstream end of the BCAL are met by the Forward Calorimeter (FCAL) covering polar angles down to $1^{\circ}$. Both of these detectors are described in the sections below. 


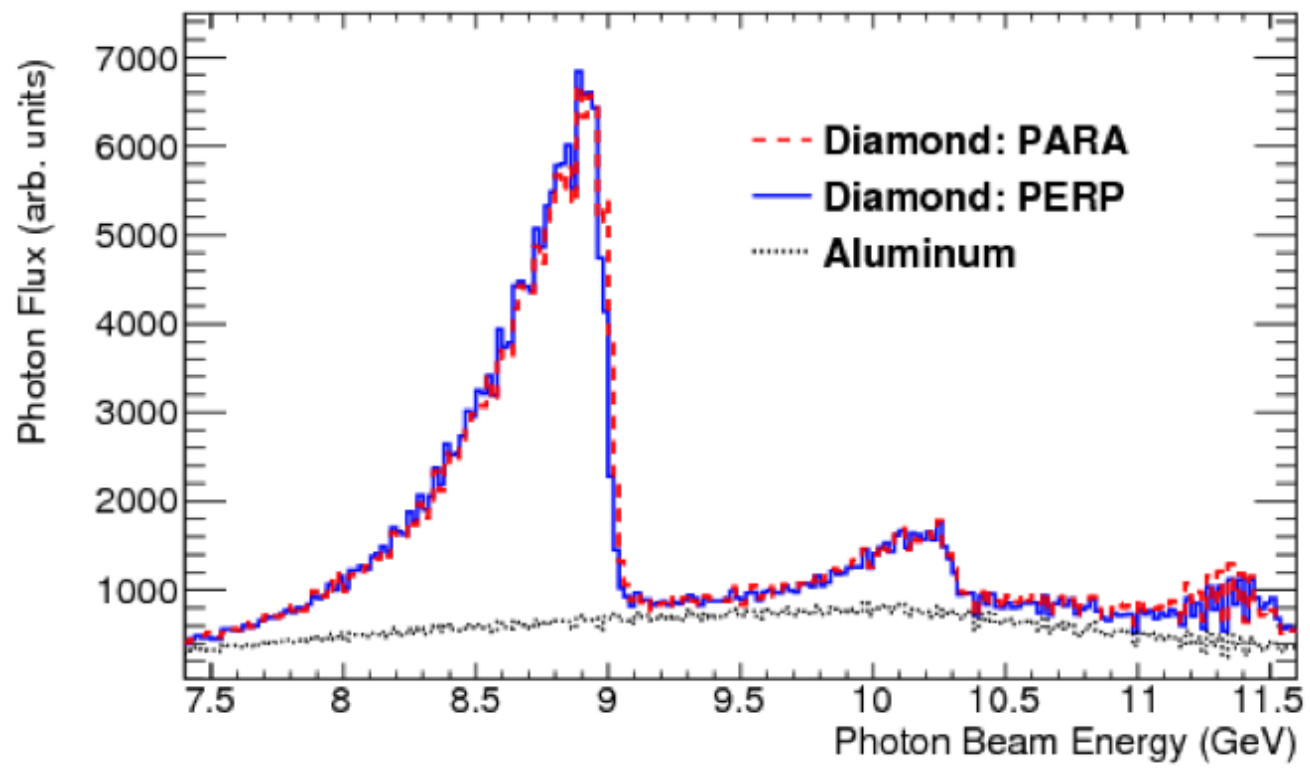

Figure 2.12: Photon beam flux as measured by the PS as a function of energy. The coherent peak at $9 \mathrm{GeV}$ is clearly seen. Reproduced from [23].

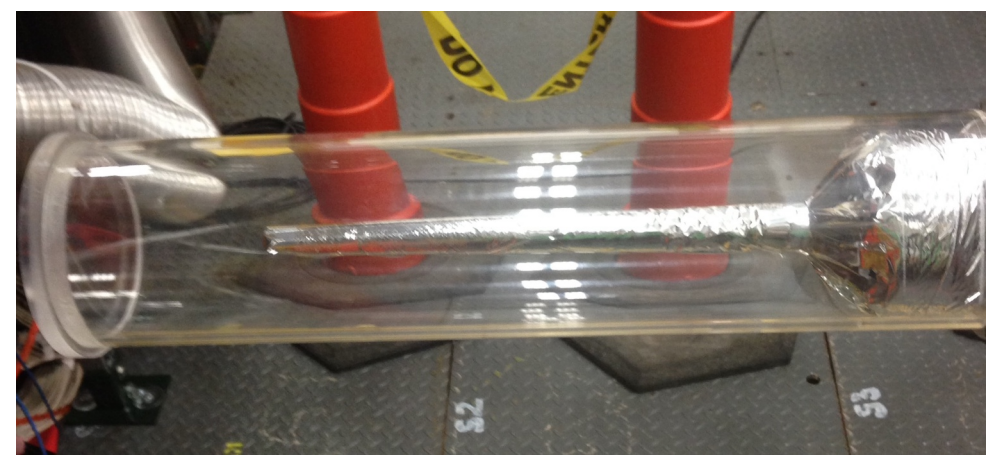

Figure 2.13: The liquid hydrogen target cell for the GlueX experiment. Beam enters from the right of the image.

\subsubsection{Barrel Calorimeter}

The BCAL is a hollow cylinder with an inner radius of $65 \mathrm{~cm}$ and an outer radius of $90 \mathrm{~cm}$. It is constructed of a fused matrix of scintillating fibers and lead. This dense amalgamation constitutes roughly 15 radiation lengths of material in the radial direction. All of the lead adds up to 28 tons and 2,663 $\mathrm{km}$ of scintillating fiber. Readout via silicon photomultipliers is attached to each end of the detector. This allows measurement of the position along the length of the BCAL by the time difference of the signal arrival at each end. The readout is segmented at roughly $2^{\circ}$ in $\phi$, and is also segmented in $r$ to allow 3D reconstruction of the shower profile. An overview of the detector can be found in Figure 2.15. The timing resolution for charged and neutral showers can be found in Figure 2.16. The mass resolution for the inclusive production of $\pi^{0}$ and $\eta$ meson production as a function of energy are found in Figure 2.17. Studies to determine the individual photon resolutions and detection efficiency are currently ongoing within the collaboration. More details of the detector 


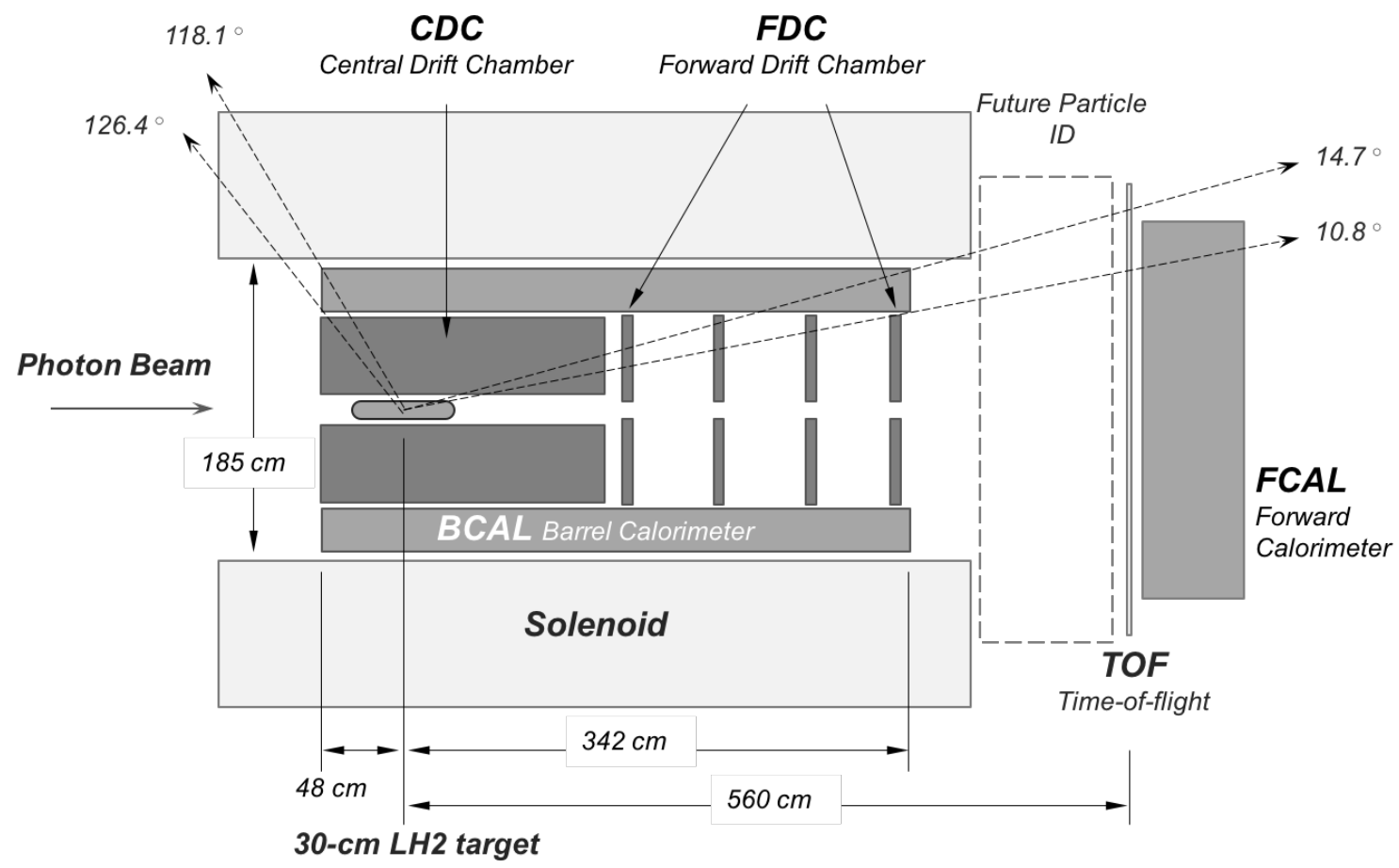

Figure 2.14: Schematic of the layout of the baseline GlueX detectors.

design and construction can be found in [16].

\subsubsection{Forward Calorimeter}

The FCAL is made up of 2800 lead-glass blocks, each $4 \times 4 \times 45 \mathrm{~cm}^{3}$, stacked behind the Time-ofFlight detector in a circular pattern. Electromagnetic showers in the lead glass produce Cherenkov light that is detected by a photomultiplier attached to the back of each block. The amount of light produced in the block is roughly proportional to the energy deposited in the block, and therefore can be used to measure the energy of the shower. The showers typically spread over multiple blocks in the FCAL. By combining information from these channels as a reconstructed shower, the energy, position, and arrival time of the shower can be determined. A picture of the stacked FCAL before installation of the dark-room enclosure that surrounds the detector can be found in Figure 2.18. The resolution of the detector for $\pi^{0}$ reconstruction can be found in Figure 2.19. More details of the detector design and construction can be found in [16].

\subsection{Charged Particle Tracking}

One of the primary capabilities of the GlueX detector is nearly hermetic charged particle tracking. This is achieved by way of two detectors, the Central Drift Chamber (CDC) and the Forward Drift Chamber (FDC). The layout of the tracking detectors is shown in Figure 2.14. The CDC is a straw tube chamber covering polar angles between $6^{\circ}$ and $168^{\circ}$, with optimal coverage between $29^{\circ}$ and $132^{\circ}$. The FDC is a cathode strip chamber covering polar angles below $20^{\circ}$. The details of each of these detectors are given below. 


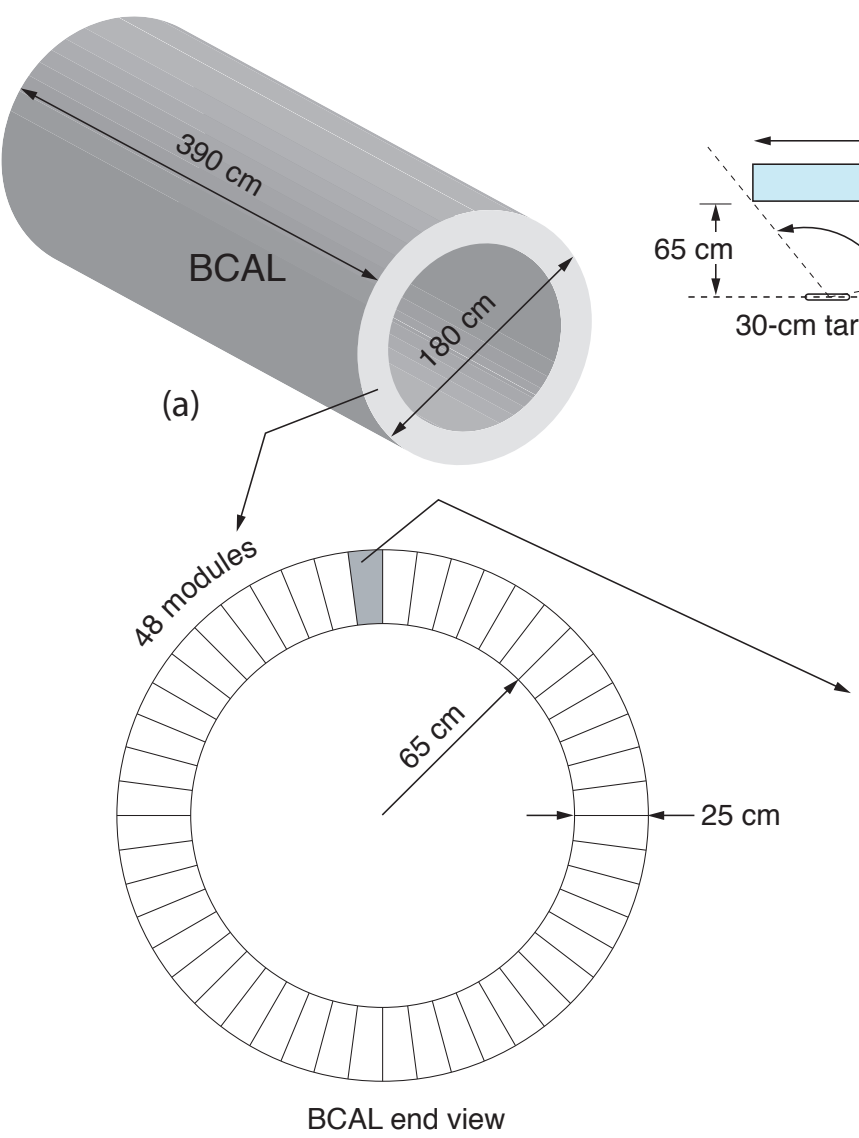

(c)

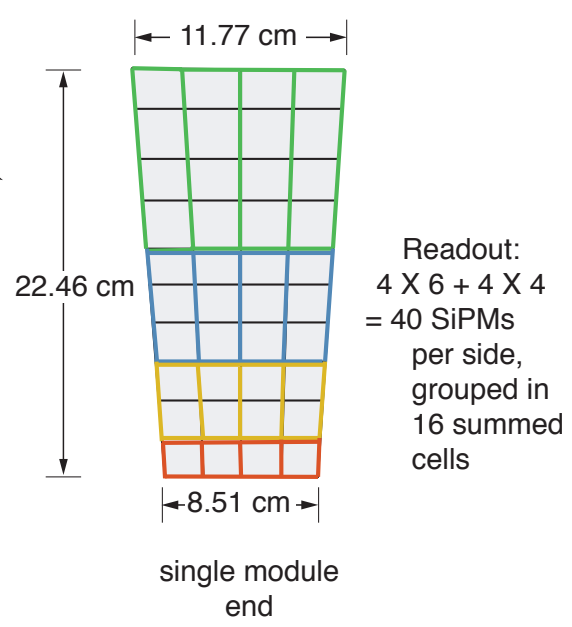

(d)

Figure 2.15: Overview of BCAL detector. (a) An isometric view of the detector. (b) Demonstration of the angular coverage relative to the target. (c) Layout of readout modules. (d) Overview of detector readout module showing grouping of individual SiPMs into readout layers. Reproduced from [16].

\subsubsection{Central Drift Chamber}

The straw tubes of the $\mathrm{CDC}$ are each $0.775 \mathrm{~cm}$ in radius and $150 \mathrm{~cm}$ long. A $20 \mu \mathrm{m}$ diameter wire runs down the center of each tube. The wires are held under tension in order to minimize displacements due to gravitational effects. The straw tubes are fixed at their ends by the crimp pin assembly for the wires at each end of the tube. The straws are glued to their neighbors within their ring at three points along the length of the detector. This should provide enough structural rigidity to the assembly to fix the geometry of the wire relative to the straw tube (more on this later in Section 3.1).

This wire is held at a voltage of $+2125 \mathrm{~V}$ relative to the tube surrounding it. The volume is filled with a 50:50 mixture of Argon and $\mathrm{CO}_{2}$. The gas mixture was chosen to optimize position resolution in the detector [26]. When a charged particle passes through the straw, it ionizes the gas, and the freed electrons drift towards the wire. The high field gradient near the wire causes amplification of the initial seed electrons and this charge can be measured by the readout electronics.

The CDC consists of 28 layers of closely-packed straw tubes broken into 7 "super-layers". Three of the super-layers $(1,4$,and 7$)$ are oriented parallel to the beam axis. The remaining super-layers are 


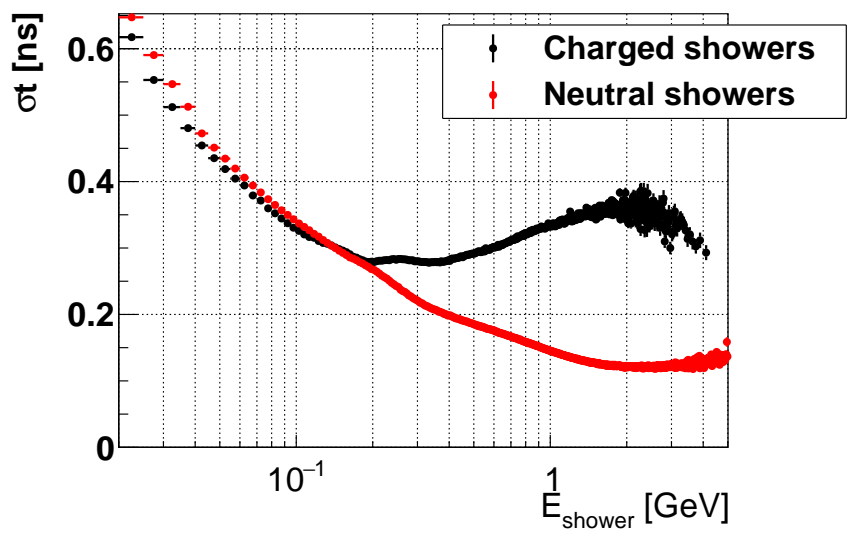

Figure 2.16: BCAL timing resolution as a function of shower energy for charged and neutral particles.

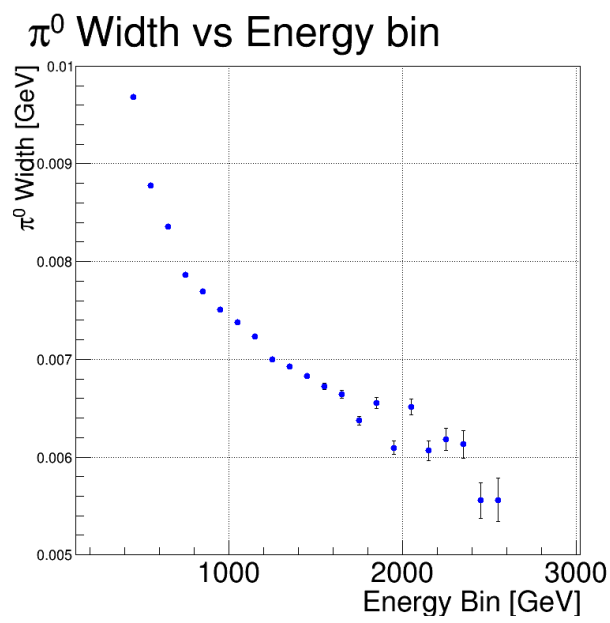

(a)

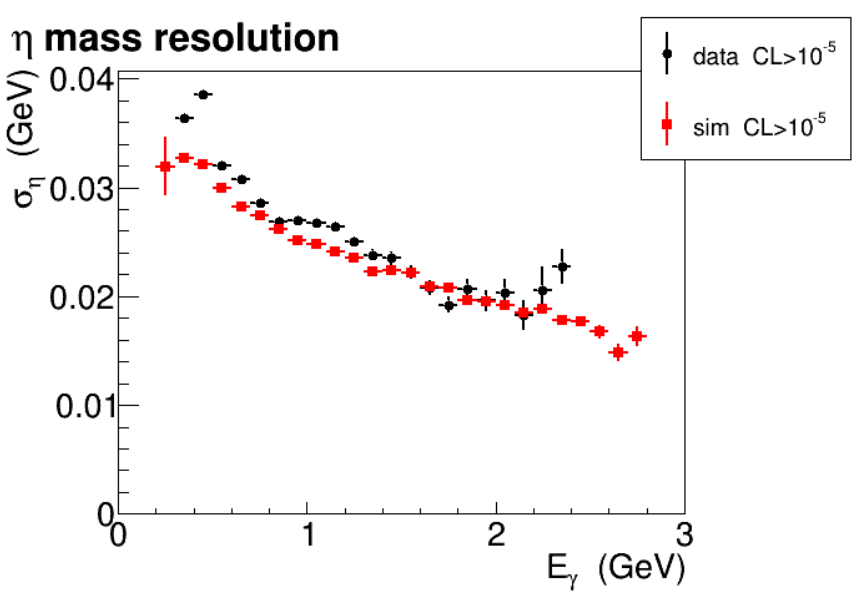

(b)

Figure 2.17: Energy resolution in the BCAL as a function of photon energy in (a) $\pi^{0}$ and (b) $\eta$ reconstruction. Pairs with nearly equal photon energy are selected in each bin.

alternately oriented at $\pm 6^{\circ}$ relative to the beam axis. A picture showing a set of alternating "stereo" layers is found in Figure 2.20. This slight skew allows position determination of track positions along the direction of the beam. In total, the CDC is comprised of 3522 wire/straw-tube assemblies.

The CDC measures the shortest drift time for ionization clusters to reach the wire. The distance of closest approach of the track to the wire is well approximated by converting measurements of the drift time of hits in each straw to distance. This drift radius defines a cylinder tangent to which the particle is known to have passed through the detector. When we fit tracks in the CDC, we are actually fitting a track to the tangents of cylinders assuming some model for propagation of the track.

The performance of the CDC after calibration is shown in Figure 2.21. The hit efficiency is very high, and begins to degrade somewhat near the edge of the straw. The spatial resolutions also have exceeded the design resolution of $150 \mu \mathrm{m}$. The poor resolution at low drift times is a characteristic of the gas mixture used and is related to the high drift velocity near the wire. The shape of this 


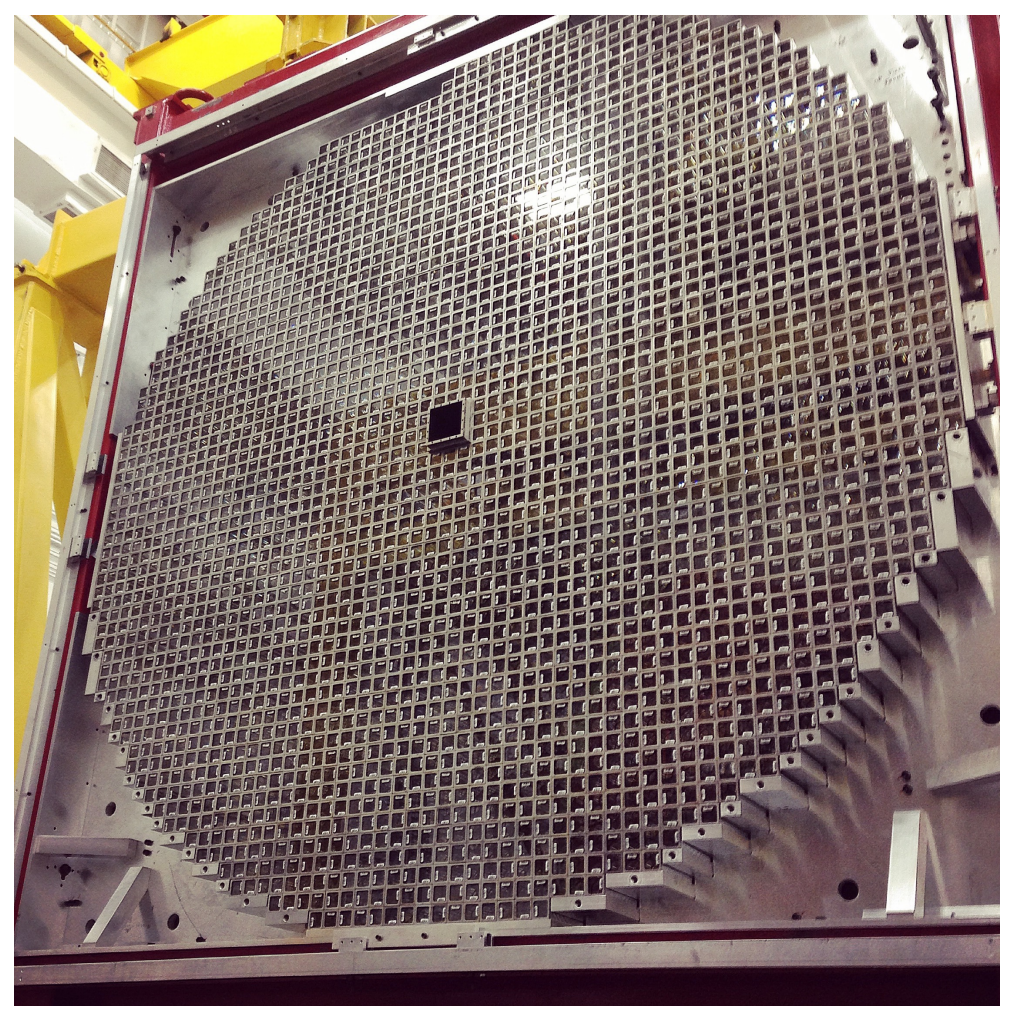

Figure 2.18: Picture of the FCAL detector before the dark room was constructed showing the individual lead-glass blocks.
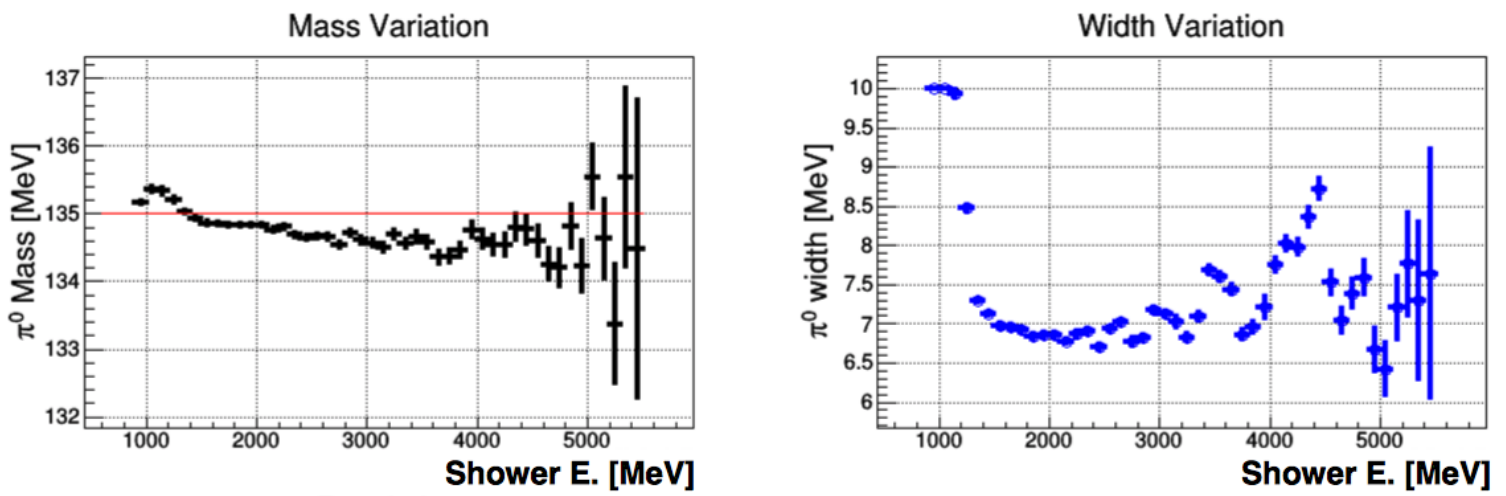

Figure 2.19: Reconstructed $\pi^{0}$ mass in the FCAL as a function of photon energy (left). Width of reconstructed $\pi^{0}$ peak versus energy (right). Reproduced from [25].

distribution is modeled in the tracking when assigning errors to the individual hits.

The CDC also provides a measurement of the energy deposition along the length of the track. The amount of energy deposited per unit length is a property of the momentum and the particle type. At low momentum, this can be used to distinguish between protons and mesons up to roughly $1 \mathrm{GeV}$. A plot showing the energy deposition per unit length for one run in the Spring 2016 period is shown in Figure 2.22. Protons can be clearly be identified in the positively-charged sample. 


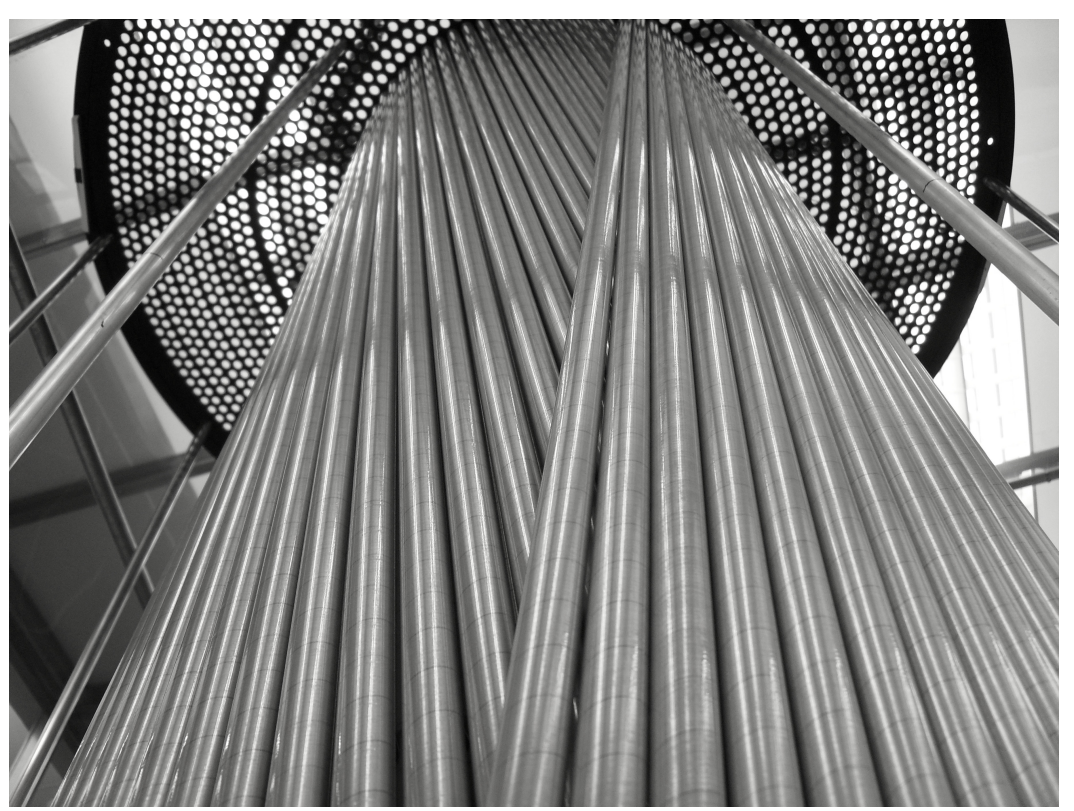

Figure 2.20: Picture showing two opposite stereo layers during construction of the CDC.

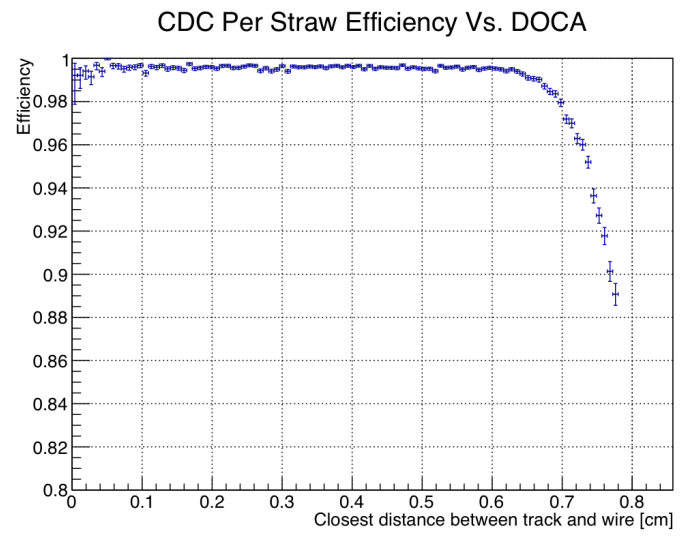

(a)

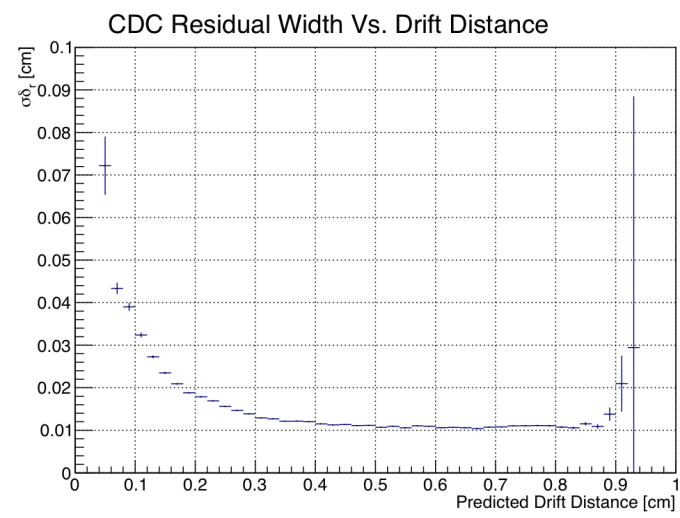

(b)

Figure 2.21: (a) CDC hit-level efficiency as a function of distance from the wire. (b) Width of biased residual distribution for cosmic tracks in the CDC.

\subsubsection{Forward Drift Chamber}

The FDC is divided into four packages of six cells each for a total of 24 layers. Each layer is rotated by $60^{\circ}$ relative to its neighbor. In each layer, a plane of wires is held between two grounded cathode planes. The wires alternate between field and sense wires with a pitch of $5 \mathrm{~mm}$. The sense wires are held at $+2200 \mathrm{~V}$ and are connected to readout electronics that record the arrival time for signals caused by ionization of the gas by charged particles. The field wires are set at negative voltage to improve the circular symmetry of the field surrounding the sense wires. The entire detector is filled with a 40:60 mixture of Argon and $\mathrm{CO}_{2}$.

What distinguishes this detector from a standard drift chamber is the addition of cathode planes on either side of the wire plane. These cathode planes are comprised of copper strips with a pitch of 
$q^{-}$

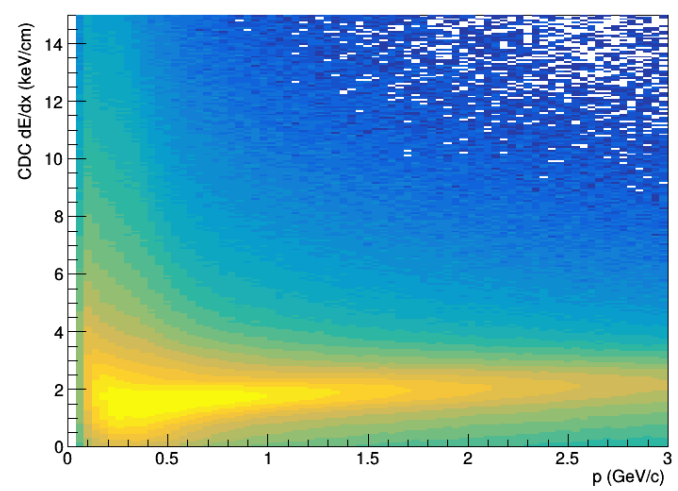

(a)

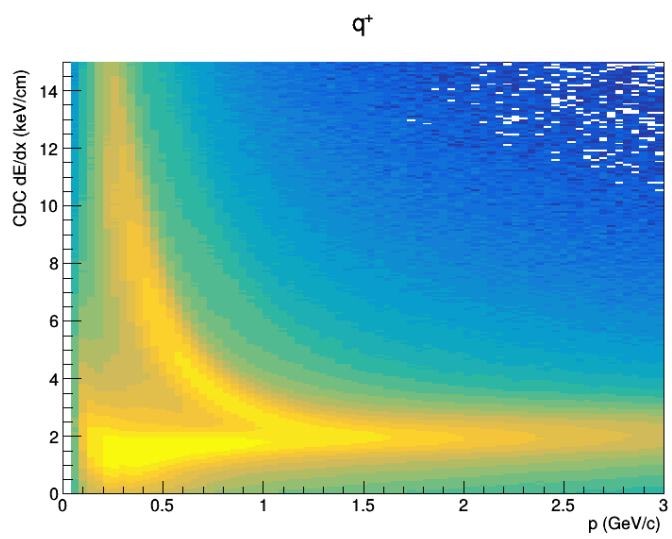

(b)

Figure 2.22: Energy deposition per unit length in the CDC for (a) negative and (b) positive charged particles. The proton band can be clearly seen.

$5 \mathrm{~mm}$ on a Kapton substrate. They are instrumented to detect the induced charge on a set of these strips. The pulse height spectrum across the strips allows determination of the avalanche position near the wires. The cathode strips are oriented at $75^{\circ}$ and $105^{\circ}$ relative to the wire direction. The charge recorded on the cathode strips is used to determine the position on each cathode plane. This information can then be combined to determine the position of the avalanche along the wire direction. The position of the avalanche in the direction perpendicular to the wire is determined with lower precision, and is useful in aligning the cathode planes to the wires (see Section 3.2). A pictorial representation of the general design of an FDC package is shown in Figure 2.23. The redundancy of three measurements to determine a $2 \mathrm{D}$ point in the detector helps to reject spurious noise hits. Information from the wires and the cathodes are both used in the tracking of charged particles.

A picture of the detector showing the four packages can be found in Figure 2.24. Each pair of cathode strips provides a spatial resolution better than $200 \mu \mathrm{m}$ in the direction along the wire. Measurements of the drift time to the sense wires provides similar resolution in the direction perpendicular to the wires with high efficiency. More details of the calibrations of the FDC can be found in Section 3.2. Details of the performance of the detector can be found in [27].

\subsection{Particle Identification Detectors}

While many of the detector systems in the experiment contribute to the identification of the reconstructed particle type, two detectors are expressly built for this purpose. These are the Start Counter (SC) and the Time-of-Flight (TOF) detectors. The SC immediately surrounds the target and records the start time for charged tracks. The TOF is located downstream of the solenoid and provides a timing measurement for forward-going particles. Both of these detectors are made of plastic scintillator that detects the passage of charged particles by producing light. A brief description of the design and performance of these detectors is provided below.

\subsubsection{Start Counter}

The SC is primarily used to select the RF bunch associated with a particular event (for more details see Section 4.3.1). It is also used as a reference time for the time-to-distance conversion in the tracking detectors. The detector is made of segmented plastic scintillator that is bent to taper 

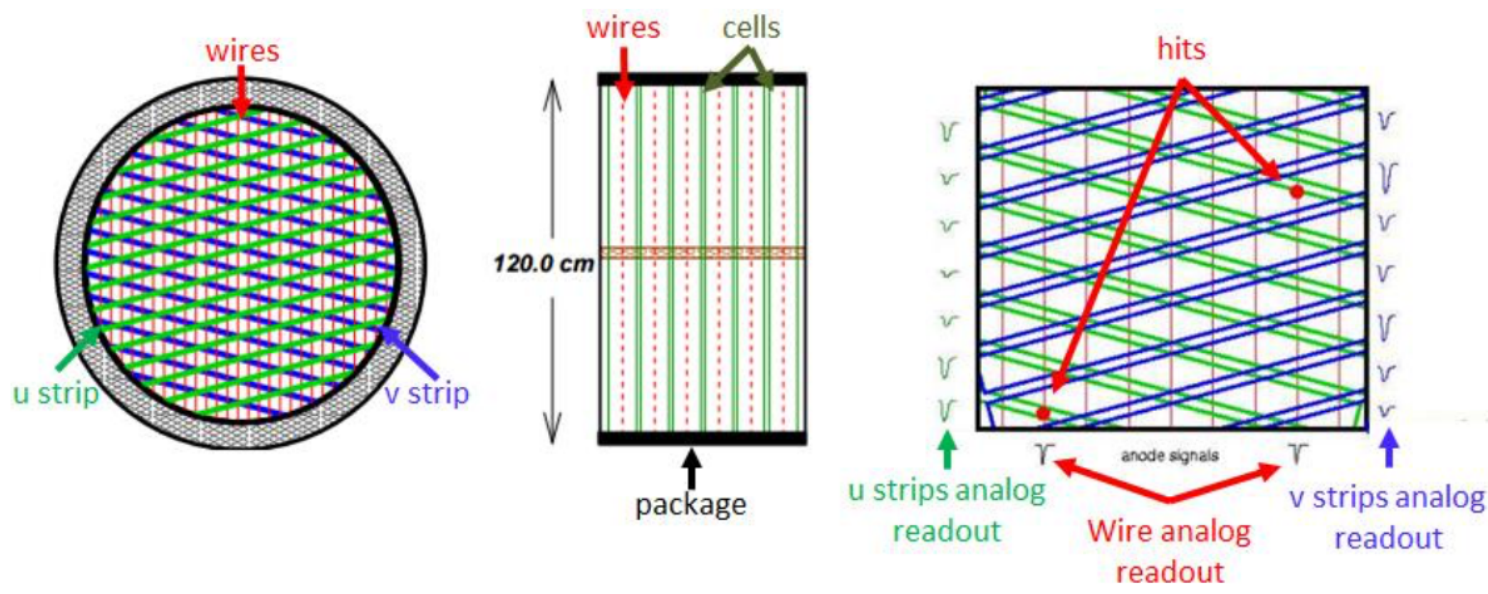

Figure 2.23: Illustration of the principle behind the FDC readout. The cathode strips are on opposite sides of the wire plane and are angled at $75^{\circ}$ and $105^{\circ}$ relative to the wire direction.

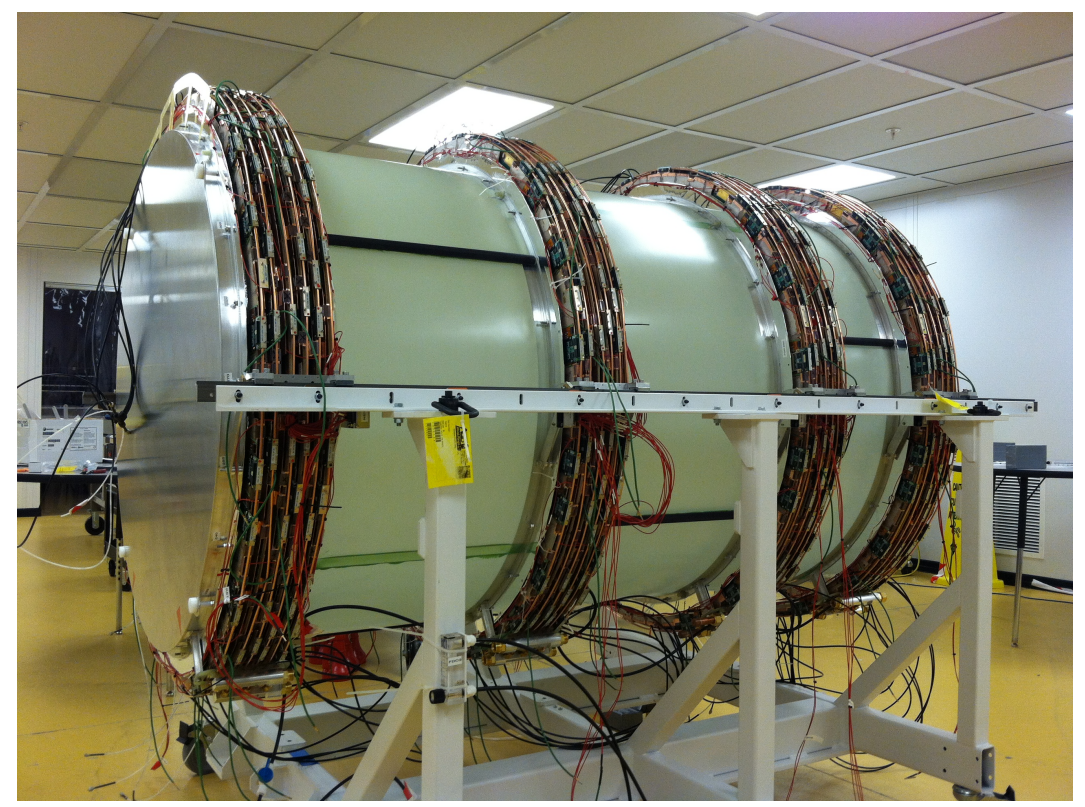

Figure 2.24: Picture of the completed FDC detector before installation into the solenoid.

around the target cell. A CAD drawing of the SC assembly can be found in Figure 2.25. The detector has a timing resolution of roughly $300 \mathrm{ps}$ after correcting for the position of the track, exceeding the design goal of $350 \mathrm{ps}$. A plot showing the timing performance of the individual sectors can be found in Figure 2.26. For more details on construction and performance of the SC, see [28].

\subsubsection{Time of Flight}

The Time of Flight detector (TOF) is a large wall of scintillating bars placed at the exit of the solenoid shown in Figure 2.27. It is designed to measure the flight time of charged particles exiting the solenoid after passing through the FDC packages. With the exception of bars closest to the beamline, 


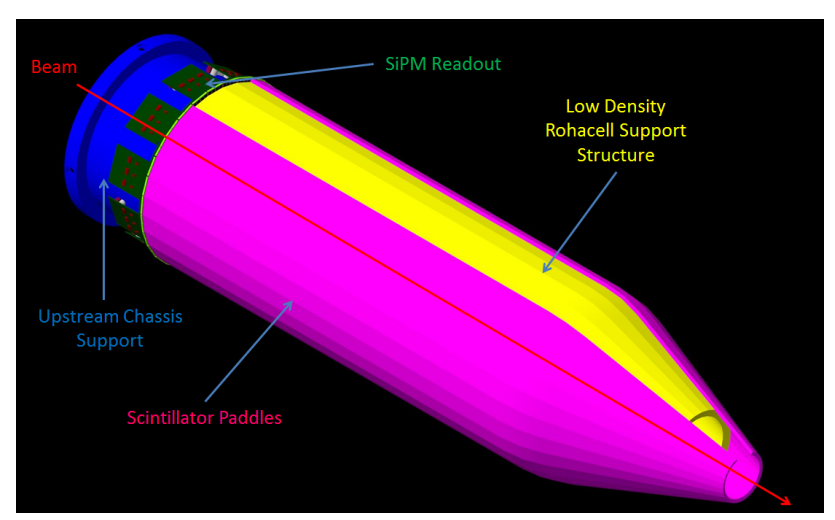

Figure 2.25: CAD drawing of SC assembly. Reproduced from [16].

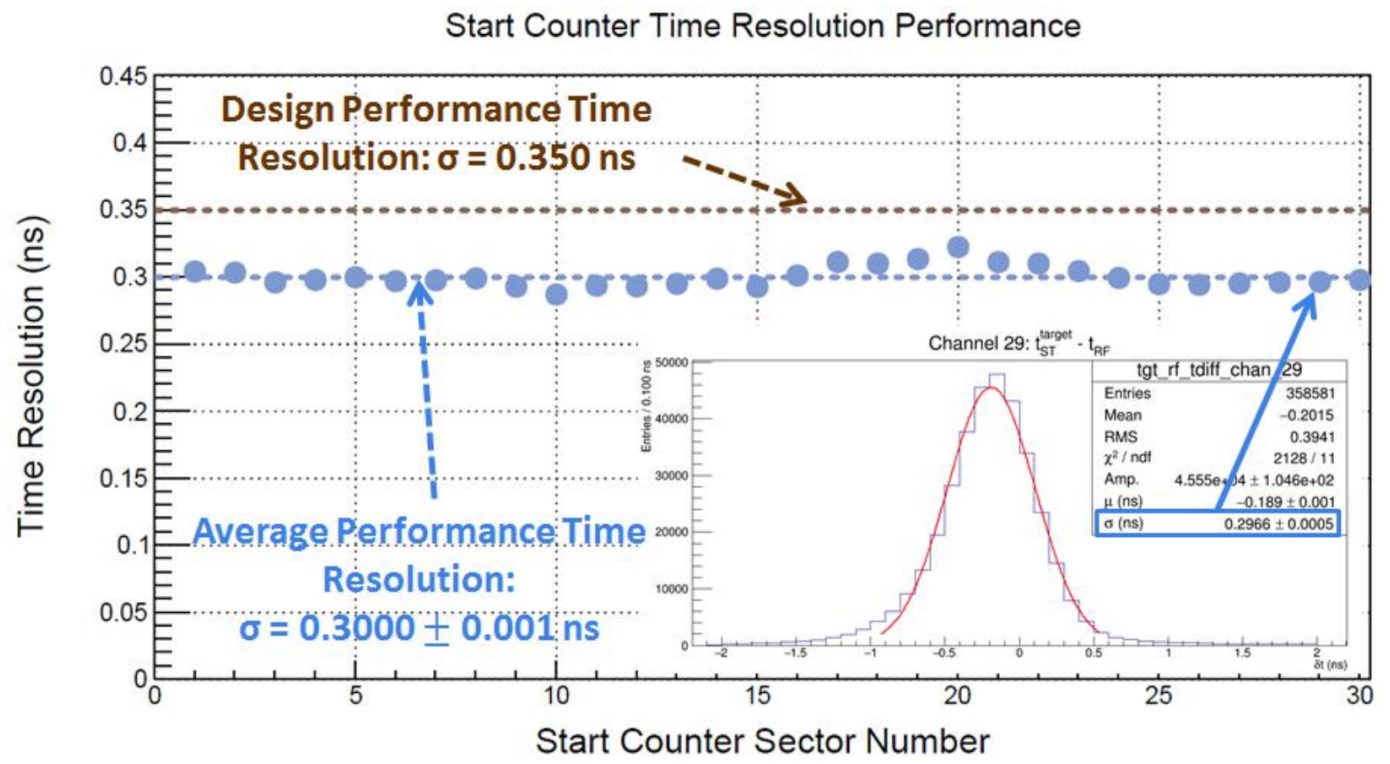

Figure 2.26: Timing resolution for each of the individual SC sectors. Reproduced from [28].

each bar is instrumented on both ends allowing reconstruction of the position along the bar. By combining the path length of the particle found using the tracking with the timing information from the TOF, we can compare the expected flight time for a given particle species against the observed time-of-flight to reject misidentified particle hypotheses. A plot of the $\beta$ versus $\mathrm{p}$ distribution is shown in Figure 2.28. The TOF provides reasonable $\pi / K$ separation for particle momenta up to 2 $\mathrm{GeV}$, and $\pi / \mathrm{p}$ separation up to $4 \mathrm{GeV}$. More information on the timing characteristics of the TOF paddles may be found in Reference [29].

\subsection{Spring 2016 Physics Trigger}

As the Spring 2016 run was technically an engineering run for the experiment, the exact configuration of the experimental trigger was continuously changing. Conditions of the trigger thresholds were stable through the set of runs presented in this thesis, but were later adjusted to optimize 


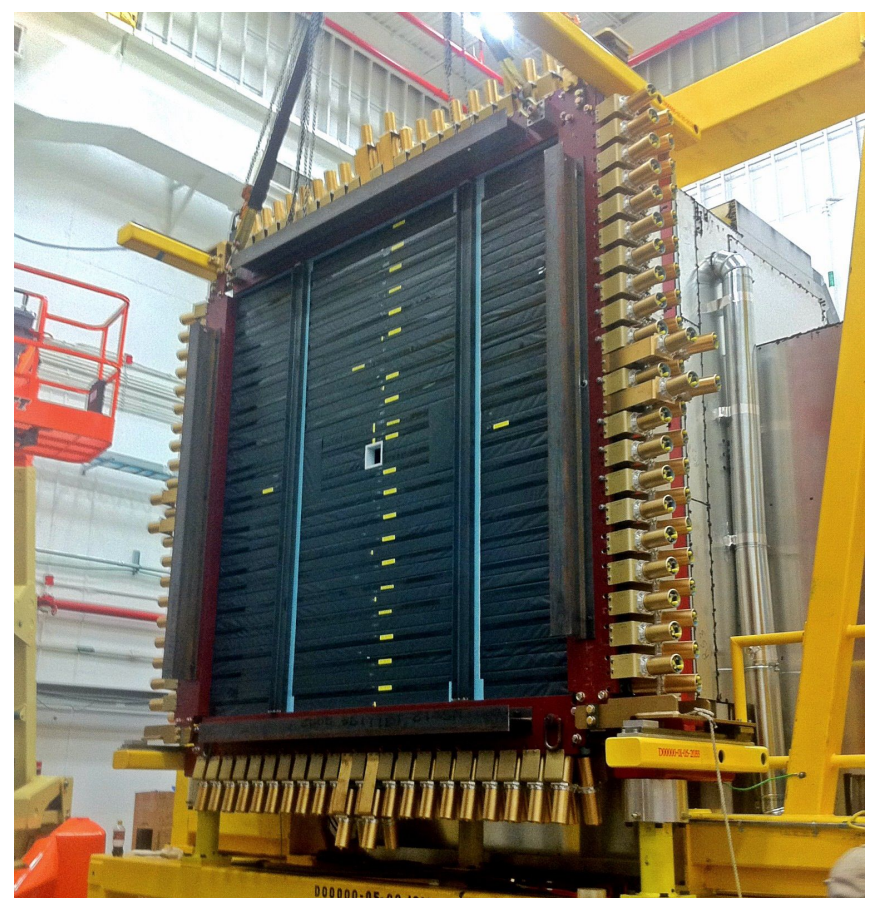

Figure 2.27: The TOF detector mounted on its support frame.

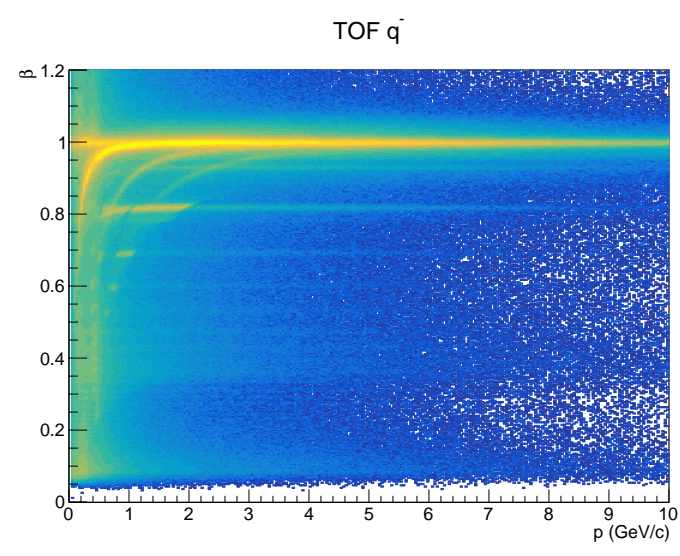

(a)

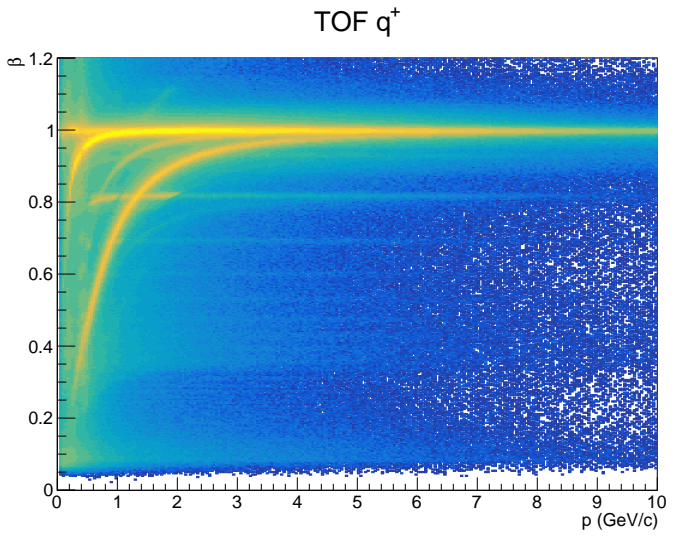

(b)

Figure 2.28: Relativistic velocity $\beta$ versus particle momentum for (a) negative and (b) positive charged tracks. Bands corresponding to electrons, pions, kaons, and protons are clearly seen. The band at $\beta \approx 0.8$ comes from misidentified RF times described in more detail in Section 4.3.1.

performance. The main experimental triggers rely on energy deposits in the BCAL and FCAL and are specified in terms of integrated ADC units. An approximate conversion from integrated ADC counts to energy is used in Table 2.2 describing the trigger settings used to collect the data in this thesis. Due to high rates, inner layers of the FCAL near the beamline are masked in some of the triggers. The number of masked layers is indicated in the table.

For $\omega$ photoproduction, it is expected that these triggers should be fairly efficient since the dominant decays contain photons that deposit large amounts of energy in the calorimeters. In 


\begin{tabular}{|c|c|c|}
\hline Trigger Threshold & $\begin{array}{c}\text { Masked } \\
\text { FCAL Layers }\end{array}$ & Notes \\
\hline \hline$E_{F C A L}+2.5 E_{B C A L}>0.50 \mathrm{GeV}$ and & 4 & Main production trigger \\
$E_{F C A L}>0.05 \mathrm{GeV}$ & 2 & \\
\hline$E_{F C A L}+0.6 E_{B C A L}>0.50 \mathrm{GeV}$ & 0 & \\
\hline$E_{B C A L}>0.55 \mathrm{GeV}$ & 2 & \\
\hline$E_{F C A L}>0.25 \mathrm{GeV}$ and any SC hit & & \\
\hline
\end{tabular}

Table 2.2: GlueX trigger settings for the Spring 2016 production run period. Threshold values are approximate.

the context of the overall GlueX physics program, most of the expected final states to be studied contain final state photons, so a trigger relying on neutral energy deposition is a natural choice [30]. Currently the trigger is not implemented in the detector simulation, and no cut is placed to select a single physics trigger type in the analysis. This could lead to additional systematic uncertainties in our acceptance correction that are not explored in this thesis. Further development is currently ongoing within the collaboration to address this issue. 


\section{Chapter 3}

\section{Charged Particle Tracking Calibrations}

As GlueX has only recently come online, much of the effort involved in preparing this thesis has been in producing physics-quality data starting from raw detector data. Achieving or beating design resolutions in each detector subsystem was the drive of the collaboration in these first years. In this chapter, we provide a brief sketch of some of the methods developed and deployed to achieve this goal, specifically for calibrations of the tracking detectors in the experiment. The design of this chapter is somewhat pedagogical, and a few more-or-less simple examples are given that may aid future calibration efforts within the collaboration.

\subsection{CDC Straw Deformations}

It was quickly noted during early operations that something was amiss with the drift time distribution in the CDC. The straw-tube design of this detector should result in a well-understood drift time spectrum. In principle, there should be a sharp leading edge indicating when particles pass closest to the wire, and a sharp falling edge when we reach the edge of the straw. In data, this behavior was observed on some wires, but others were found to be without the sharp falling edge characteristic of the idealized straw geometry. An example of two "good" wires can be found at the top of Figure 3.1. Unfortunately, most of the wires in the CDC showed similar timing spectra to the two lower plots in the figure where the sharp falling edge is missing.

The strange behavior was determined to be consistent with the hypothesis of deformations of the straw tubes themselves. The wires and straws of drift tubes are known to experience gravitational sag which can be described as in Reference [31], but the effect can be mitigated by careful design. During the assembly of the CDC, tension was carefully applied to the wires before crimping them into place. This tension was also verified by in situ measurements after construction was completed. We believe the wire positions in the CDC are fairly well determined. As mentioned in Section 2.4.1 the position of the straw tubes at their ends should be well determined since they are fixed to the support frame. However, it seems that near the middle of the detector the straw tubes have some deformation. This deformation skews the time-to-distance relation near the center of the straw. In areas where the straw tube is farther than the nominal distance of $0.775 \mathrm{~cm}$ from the wire, the electric field is distorted, and the drift velocity of electrons in the gas will be slower, resulting in longer maximum drift times to the edge of the straw. On the opposite side of the straw, the drift velocity will be increased, and shorter drift times are measured. If one looks carefully at the lowerleft plot of Figure 3.1 you can make out two falling edges in the drift time. This may correspond to two opposite sides of the straw having different time-to-distance relations. This must be accounted 
for in our analysis in order to achieve optimal reconstruction of the drift distance in each straw.

A depiction of the local coordinate system of a single CDC straw is shown in Figure 3.2. Here the $\mathrm{x}$-axis is parallel to the ground, and the $\mathrm{y}$-axis points upwards. The line of closest approach (LOCA) is perpendicular to both the wire and the track passing the wire. We define the angle $\phi_{L O C A}$ as the angle between the LOCA and the local straw coordinate $x$-axis. To determine the deformation of each straw, we fit straight cosmic tracks in the CDC. The predicted LOCA to a given wire based on the track and the corresponding $\phi_{L O C A}$ is then calculated. If there is a hit on the wire, we fill a histogram of the predicted drift distance versus $\phi_{L O C A}$ as in Figure 3.3. The upper value determined in a fit to the edge of the distribution in each bin of $\phi_{L O C A}$ should correspond to the location of the edge of the straw in this direction. We then fit these edge positions to a function of the form

$$
f\left(\phi_{L O C A}\right)=c_{0}+c_{1} \cos \left(\phi_{L O C A}+c_{2}\right) .
$$

The parameter $c_{0}$ may be identified as the average radius of the straw, $c_{1}$ is the magnitude of the deformation, and $c_{2}$ is the negative of the direction of maximal deformation. A plot of these parameters for fits to all straws in the CDC can be found in Figure 3.4. The average value of $c_{0}$ is consistent with the nominal radius of the straw tubes. The values of $c_{1}$ indicate that the average deformation at the center of the straw is roughly $1 \mathrm{~mm}$. The values of $c_{2}$ indicate that the majority of this deformation is pointing downwards. Since the effect is mostly in the downwards direction, gravity is likely partly to blame, but the effect is not uniform from straw to straw so the exact cause is unclear. If we look at the projection of the deformations on the $x$ and $y$-axes in Figure 3.5, it is clear that this effect varies systematically both in magnitude and direction between straws. Thus in order to provide a consistent time-to-distance lookup, we must provide a method that accounts for this variability.

The method employed is described here. At each measurement in the track fitting routine, a value $\delta$ is calculated as

$$
\delta=c_{1}\left(1-\frac{\left(z-z_{\text {center }}^{C D C}\right)^{2}}{(75.0 \mathrm{~cm})^{2}}\right) \cos \left(\phi_{L O C A}+c_{2}\right)
$$

where $z$ and $\phi_{L O C A}$ are specific to the track being fit and $c_{1}$ and $c_{2}$ are the characteristic magnitude and direction of the individual straw's deformation. The $\mathrm{z}$ dependence is modeled as a parabola with maximal offset at $z=z_{\text {center }}^{C D C}$. We then use the following model of the time-to-distance relation.

$$
d=f_{\delta}\left(\frac{d_{0}}{f_{0}} * P+1-P\right)
$$

where

$$
P= \begin{cases}0 & \text { if } t>250 \mathrm{~ns} \\ \frac{250 .-t}{250 .} & \text { otherwise }\end{cases}
$$

The value $d_{0}$ is interpolated from a time-to-distance table for a straw with zero deformation based on the drift time. If $\delta>0$, we are on the side of the straw with larger than normal wire to straw distance. In this regime we calculate

$$
\begin{aligned}
f_{\delta} & =a \sqrt{t}+b t+c t^{3} \\
f_{0} & =a_{1} \sqrt{t}+b_{1} t+c_{1} t^{3} \\
a & =a_{1}+a_{2}|\delta| \\
b & =b_{1}+b_{2}|\delta| \\
c & =c_{1}+c_{2}|\delta|+c_{3} \delta^{2} .
\end{aligned}
$$



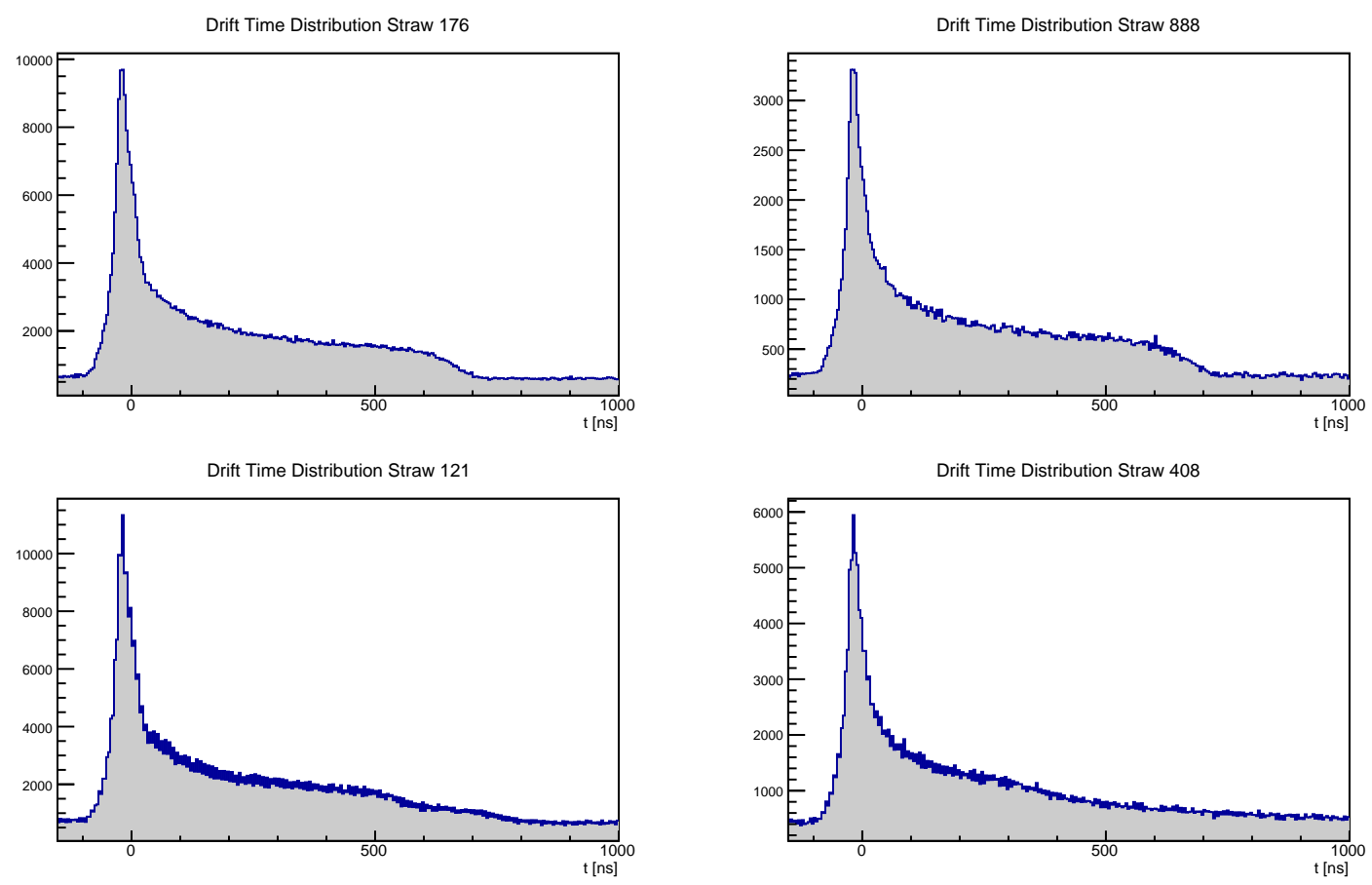

Figure 3.1: Distribution of measured drift times for four individual straws in the CDC. The top two plots are for straws with typical drift time distributions and exhibit a sharp falling edge at $t_{\max }$. The long tails in the bottom plots are atypical.

When $\delta$ is negative we calculate

$$
\begin{aligned}
f_{\delta} & =a \sqrt{t}+b t \\
f_{0} & =a_{1} \sqrt{t}+b_{1} t \\
a & =a_{1}+a_{2}|\delta|+a_{3} \delta^{2} \\
b & =b_{1}+b_{2}|\delta|+b_{3} \delta^{2} .
\end{aligned}
$$

These functional forms were chosen through a study of simulated time-to-distance behavior in the CDC straws with varying wire offsets [32]. The initial $a_{i}, b_{i}$, and $c_{i}$ values were chosen based on this simulation. In Figure 3.6 we show that this functional form can be used in a fit to the predicted time-to-distance from the track fit in order to improve the parameters determined by simulation. This fitting method is applied to each of the runs in the Spring 2016 data set in order to determine the parameters for the time-to-distance lookup. 


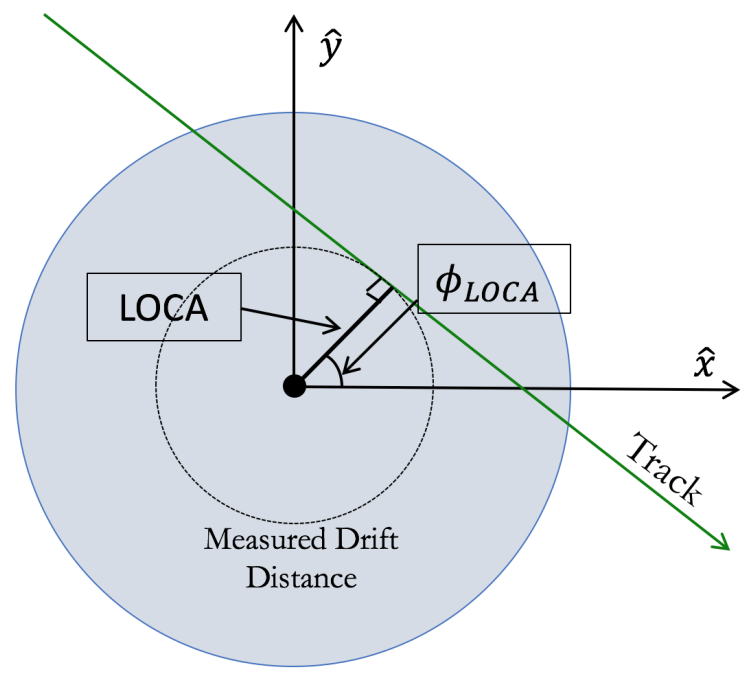

Figure 3.2: Local CDC straw coordinates. $\phi_{L O C A}$ is the angle between the measured track and the perpendicular line of closest approach between the track and the wire.

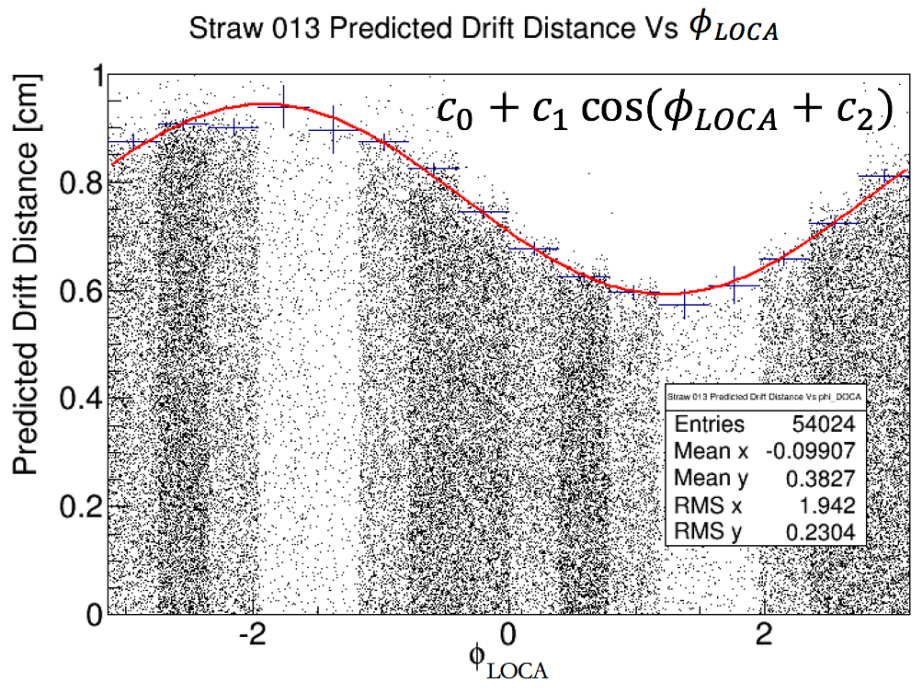

Figure 3.3: Predicted drift distance for which a hit in the straw is detected versus $\phi_{L O C A}$.

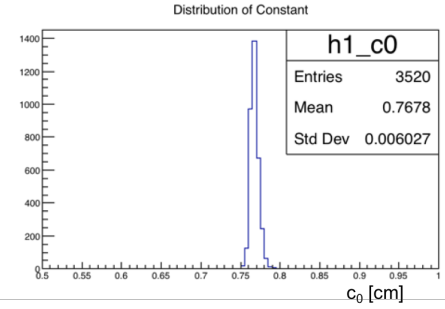

(a)

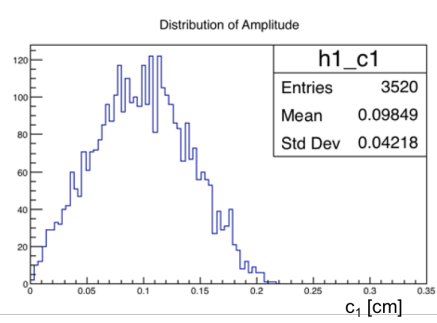

(b)

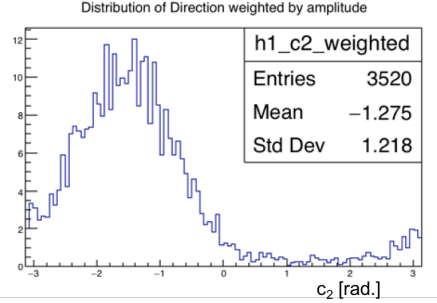

(c)

Figure 3.4: Fit values for CDC straw deformations: (a) parameter $c_{0}$ (b) parameter $c_{1}$ and (c) parameter $c_{2}$ weighted by parameter $c_{1}$. 


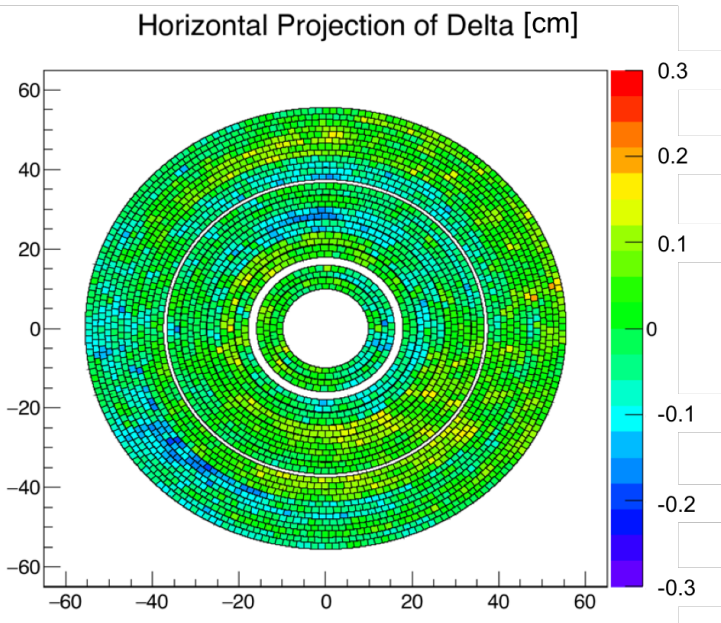

(a)

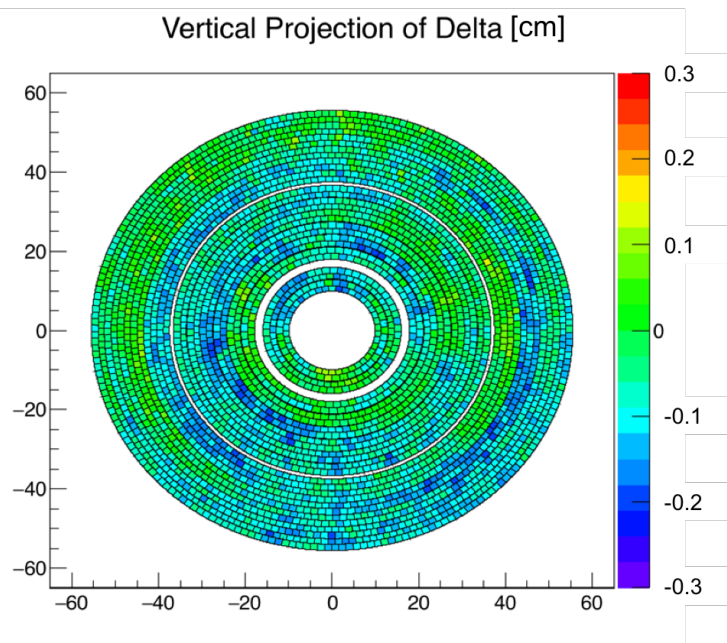

(b)

Figure 3.5: Projection of the maximal straw deformation $\delta$ onto the (a) horizontal and (b) vertical axes. Most of the deformation seems to occur in the stereo layers, though this is not without exception.

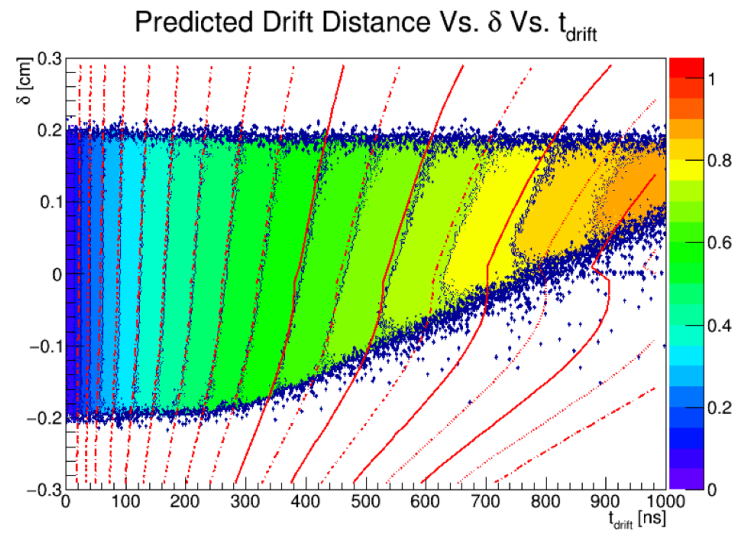

(a)

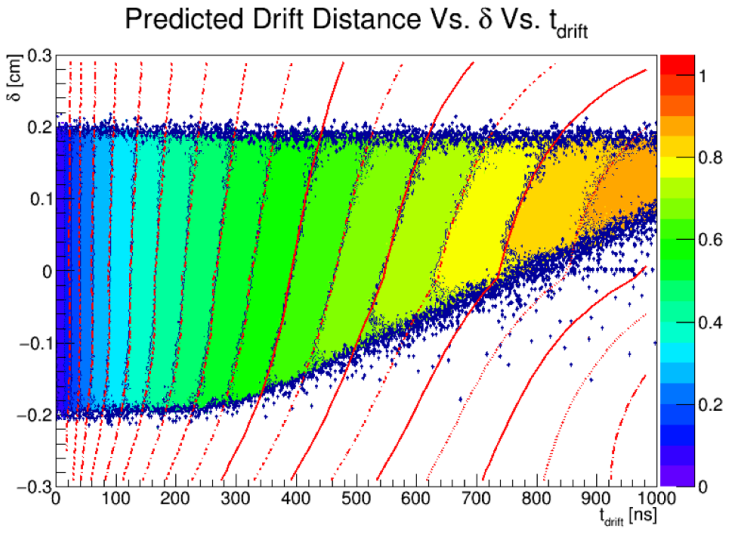

(b)

Figure 3.6: Average predicted drift distance from the fit as a function of $\delta$ and $t_{\text {drift }}$. The red contours indicate the time-to-distance lookup function. Figure (a) shows this function with values determined from simulation while (b) is the result after a fit to the data. 


\subsection{Internal FDC Alignment}

Each plane of the FDC measures a 2D point in space, with the third dimension being determined by the position and orientation of the plane itself. The $2 \mathrm{D}$ measurement is actually formed from three different measurements in the $\hat{u}, \hat{v}$, and $\hat{x}$ directions as shown in Figure 3.7. This redundancy allows us to align the cathodes of each package to the wires without the use of tracking. The cathode planes are grounded, so the charge that is seen on the strips is an image charge due to the charge collection on the wire. This amplification region is localized very close to the wire itself, so the reconstructed $\mathrm{x}$ position by the cathodes, $x_{c}$, should match the known x position of the wire, $x_{w}$. We refer to the calibration to match $x_{c}$ to $x_{w}$ as the "internal alignment" of the FDC packages.

The reconstructed $\mathrm{x}$ and $\mathrm{y}$ positions from the cathode $u$ and $v$ measurements are given as

$$
\begin{aligned}
& x_{c}=\frac{u \sin \phi_{v}-v \sin \phi_{u}}{\sin \left(\phi_{u}-\phi_{v}\right)} . \\
& y_{c}=-\frac{u \cos \phi_{v}-v \cos \phi_{u}}{\sin \left(\phi_{u}-\phi_{v}\right)} .
\end{aligned}
$$

Now we wish to apply alignment constants to these values. For example If we allow each of the angles and coordinates to shift, we may write $x_{c}$ as

$$
x_{c}=\frac{(u+\delta u) \sin \left(\phi_{v}+\delta \phi_{v}\right)-(v+\delta v) \sin \left(\phi_{u}+\delta \phi_{u}\right)}{\sin \left(\phi_{u}-\phi_{v}+\delta \phi_{u}-\delta \phi_{v}\right)} .
$$

Using the trigonometric identity $\sin (A+B)=\sin A \cos B+\cos A \sin B$ and the small angle approximations $\sin x \approx x$ and $\cos x \approx 1$ we may write

$$
x_{c} \approx \frac{(u+\delta u)\left(\sin \phi_{v}+\delta \phi_{v} \cos \phi_{v}\right)-(v+\delta v)\left(\sin \phi_{u}+\delta \phi_{u} \cos \phi_{u}\right)}{\sin \left(\phi_{u}-\phi_{v}\right)+\left(\delta \phi_{u}-\delta \phi_{v}\right) \cos \left(\phi_{u}-\phi_{v}\right)} .
$$

For reasons that will become clear later, we take the derivatives of this formula with respect to the alignment parameters in the limit the alignment parameters themselves go to zero.

$$
\begin{aligned}
\left.\frac{\partial x_{c}}{\partial \delta u}\right|_{\delta \rightarrow 0} & =\frac{\sin \phi_{v}}{\sin \left(\phi_{v}-\phi_{u}\right)} \\
\left.\frac{\partial x_{c}}{\partial \delta \phi_{u}}\right|_{\delta \rightarrow 0} & =\frac{\left[v-u \cos \left(\phi_{u}-\phi_{v}\right)\right] \sin \phi_{v}}{\left[\sin \left(\phi_{v}-\phi_{u}\right)\right]^{2}}
\end{aligned}
$$

$$
\begin{aligned}
\left.\frac{\partial x_{c}}{\partial \delta v}\right|_{\delta \rightarrow 0} & =-\frac{\sin \phi_{u}}{\sin \left(\phi_{v}-\phi_{u}\right)} \\
\left.\frac{\partial x_{c}}{\partial \delta \phi_{u}}\right|_{\delta \rightarrow 0} & =\frac{\left[u-v \cos \left(\phi_{u}-\phi_{v}\right)\right] \sin \phi_{u}}{\left[\sin \left(\phi_{v}-\phi_{u}\right)\right]^{2}}
\end{aligned}
$$

These formulas can be used to align the cathodes to the wires. A plot of the average value of the residual $r=x_{c}-x_{w}$ as a function of the $x_{c}-y_{c}$ location on the FDC plane for all 24 cells is shown in Figure 3.8. The method of aligning the cathodes to the wires proceeds as follows. We fit each of these 2D histograms with a plane:

$$
f(x, y)=c_{0}+c_{1} x+c_{2} y
$$

In order to determine the alignment parameters indicated by this fit, we need to carefully consider what causes the shape of this distribution. According to equation 3.5 we have

$$
x_{c}=x_{c}\left(u, v, \phi_{u}, \phi_{v}, \delta u, \delta v, \delta \phi_{u}, \delta \phi_{v}\right)
$$

We may define the following

$$
\widehat{x_{c}} \equiv x_{c}\left(u, v, \phi_{u}, \phi_{v}, \widehat{\delta u}, \widehat{\delta v}, \widehat{\delta \phi_{u}}, \widehat{\delta \phi_{v}}\right)
$$

where $\widehat{\delta u}, \widehat{\delta v}, \widehat{\delta \phi_{u}}$ and $\widehat{\delta \phi_{v}}$ are the alignment parameters needed to make $\widehat{x_{c}}$ equal to $x_{w}$, i.e. $r=\widehat{x_{c}}-x_{w}=0$. In reality the four alignment constants here are one more than is necessary to 


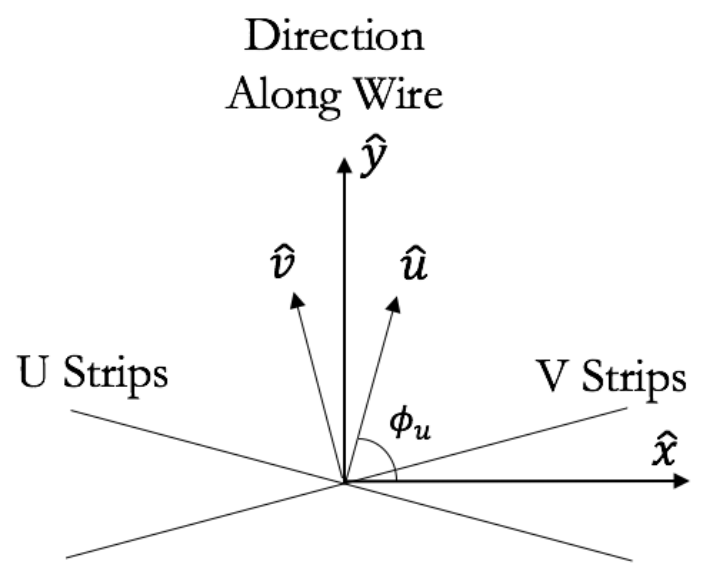

Figure 3.7: The local FDC coordinate system.

describe the possible misalignment. As such, we set $\widehat{\delta v}=0$. The choice is arbitrary. Now, we Taylor expand $x_{c}$ about $\widehat{x_{c}}$ as

$$
x_{c} \approx \widehat{x_{c}}+\frac{\partial x_{c}}{\partial \delta u} \widehat{\delta u}+\frac{\partial x_{c}}{\partial \delta \phi_{u}} \widehat{\delta \phi_{u}}+\frac{\partial x_{c}}{\partial \delta \phi_{v}} \widehat{\delta \phi_{v}}+\cdots
$$

We then have

$$
r=x_{c}-x_{w} \approx \frac{\partial x_{c}}{\partial \delta u} \widehat{\delta u}+\frac{\partial x_{c}}{\partial \delta \phi_{u}} \widehat{\delta \phi_{u}}+\frac{\partial x_{c}}{\partial \delta \phi_{v}} \widehat{\delta \phi_{v}} .
$$

This can now be equated to our fit result and replacements made for the derivatives calculated in equations 3.6-3.9.

$c_{0}+c_{1} x_{c}+c_{2} y_{c}=\frac{\sin \phi_{v}}{\sin \left(\phi_{v}-\phi_{u}\right)} \widehat{\delta u}+\frac{\left[v-u \cos \left(\phi_{u}-\phi_{v}\right)\right] \sin \phi_{v}}{\left[\sin \left(\phi_{v}-\phi_{u}\right)\right]^{2}} \widehat{\delta \phi_{u}}+\frac{\left[u-v \cos \left(\phi_{u}-\phi_{v}\right)\right] \sin \phi_{u}}{\left[\sin \left(\phi_{v}-\phi_{u}\right)\right]^{2}}$

We are almost there. We can identify immediately

$$
c_{0}=\frac{\sin \phi_{v}}{\sin \left(\phi_{v}-\phi_{u}\right)} \widehat{\delta u} \rightarrow \widehat{\delta u}=c_{0} \frac{\sin \left(\phi_{v}-\phi_{u}\right)}{\sin \phi_{v}} .
$$

The remaining two terms have dependence on $u$ and $v$. Inverting equations 3.3 and 3.4 yields

$$
\begin{aligned}
& u=-\left(x_{c} \cos \phi_{u}+y_{c} \sin \phi_{u}\right) \\
& v=-\left(x_{c} \cos \phi_{v}+y_{c} \sin \phi_{v}\right) .
\end{aligned}
$$

This leads to a coupled set of equations for $\widehat{\delta \phi_{u}}$ and $\widehat{\delta \phi_{v}}$ that can be solved by standard techniques.

We now have our estimates for the alignment parameters based on the fits to our data. These can then be applied to the calibrations database. The results for the average residual after of a single pass of this technique are shown in Figure 3.9.

There is an additional step needed to determine a starting point for the cathode strip pitch values. The cathode planes in the FDC are pieced together from three panels of readout on Kapton substrate. Due to mechanical stresses in the mounting procedure, the pitch of the individual cathode strips may vary slightly. In addition, small variations in the width of the seam between the three panels can affect the measurement. This effect can be measured and accounted for. To do so, we project the measurement from one cathode onto the other using the wire positions. The residual between 

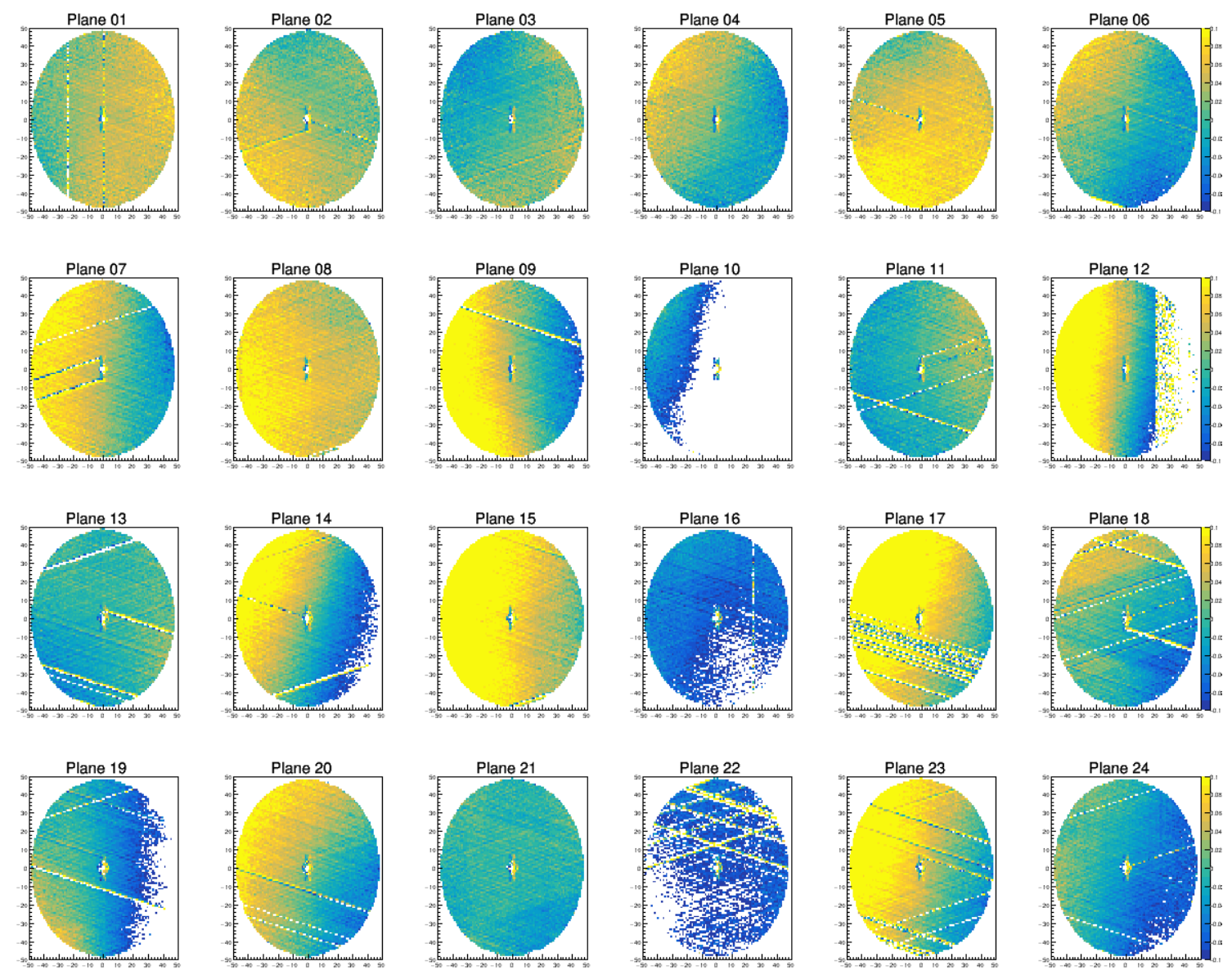

Figure 3.8: Average distance between the reconstructed wire position from the cathodes and the known wire position as a function of the $\mathrm{x}-\mathrm{y}$ position in the FDC cell before calibrations. The colored scale runs from -0.1 to $0.1 \mathrm{~cm}$. Many of the average residuals go off of the scale.

this projected position and the measured position are plotted as a function of the measured position. These distributions are fit with straight lines in the region of each of the three foil panels. These fit results are used to determine an update to the pitch parameters (related to the slope) and the gap between panels (related to the intercept of the line fits at the boundary of the readout panels). The result of this alignment procedure for the cathodes is shown in Figure 3.10. This cathode pitch alignment procedure is alternated with the planar alignment procedure until convergence is reached ( $\sim 5$ iterations). The result of this phase determines one set of cathode alignment and strip pitch values for which the cathodes and wires are optimally aligned. However, there is still freedom to choose the average pitch of each cathode plane. To determine this value, we need to include information from tracks passed through multiple detector planes. This process is described later in Section 3.4.4. 

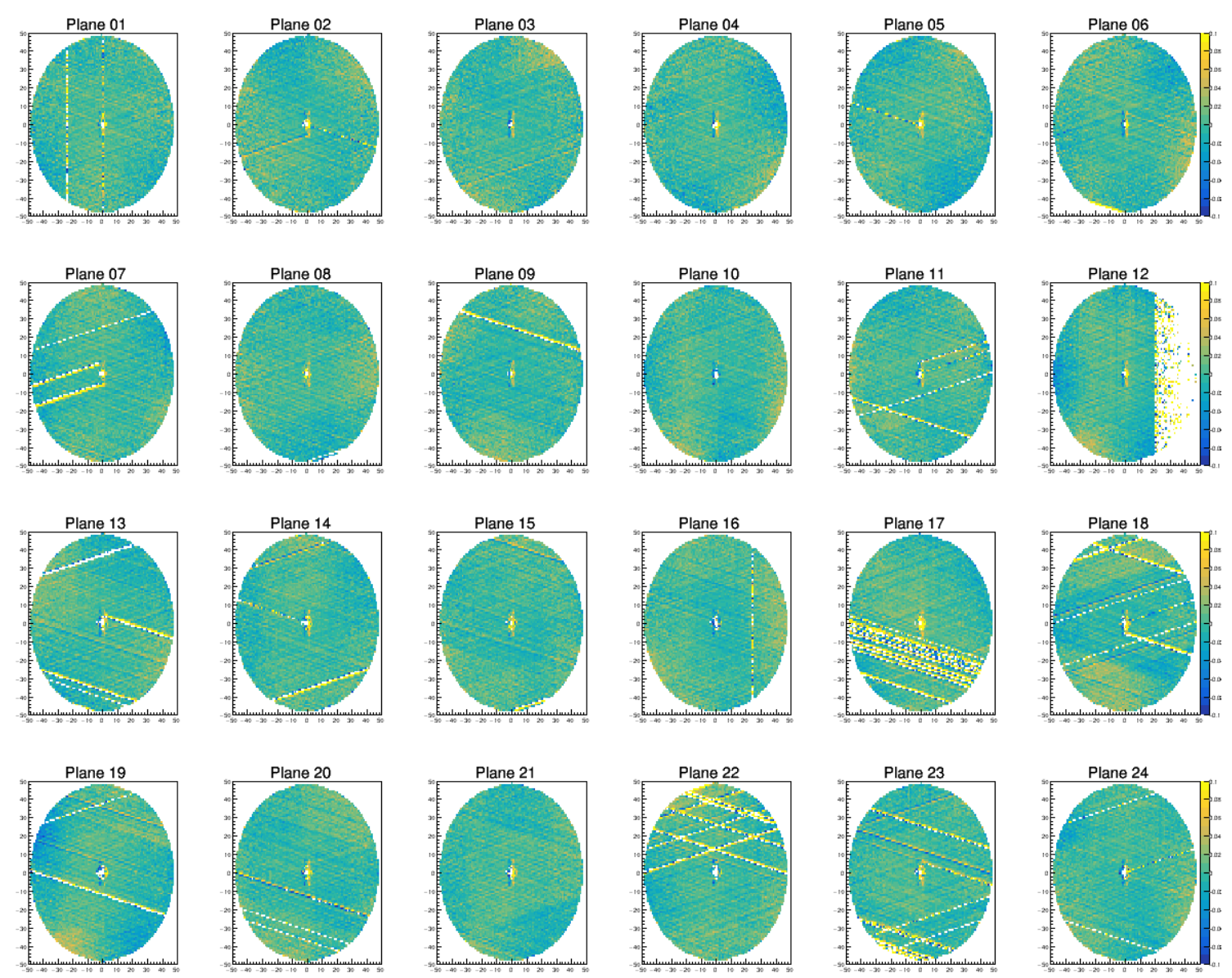

Figure 3.9: Average distance between the reconstructed wire position from the cathodes and the known wire position as a function of the $\mathrm{x}-\mathrm{y}$ position in the FDC cell after a single pass of the internal alignment procedure. The colored scale runs from -0.1 to $0.1 \mathrm{~cm}$. Plane 12 has one missing HV region due to hardware problems. 


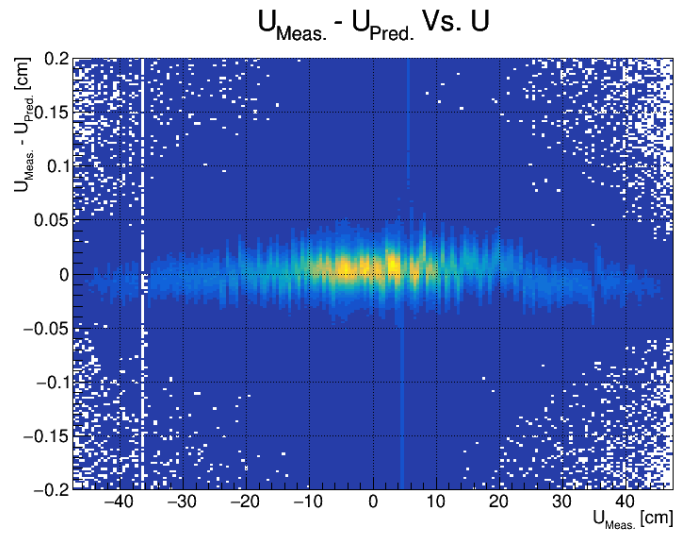

(a) U residuals before alignment.

$U_{\text {Meas. }}-U_{\text {Pred. }}$ Vs. U

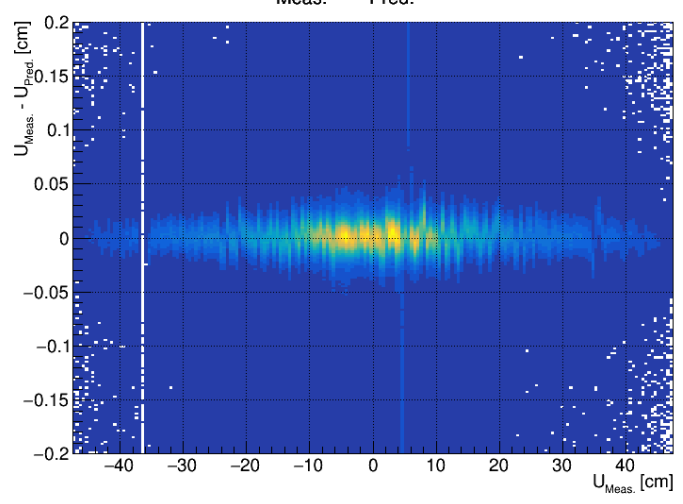

(c) U residuals after alignment.

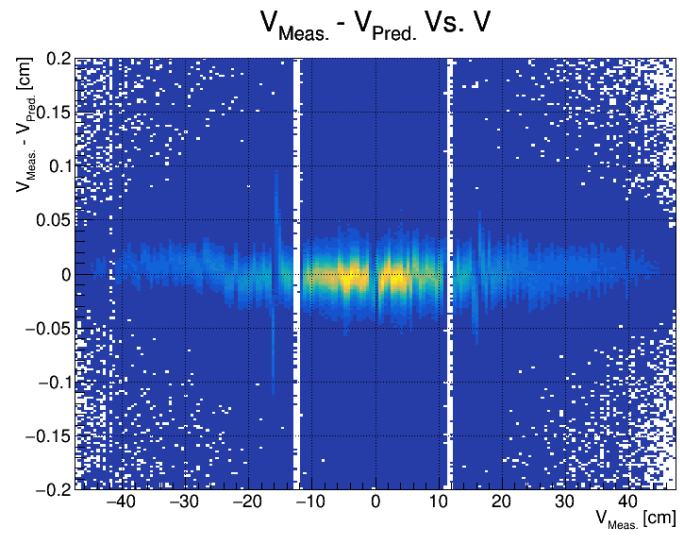

(b) $\mathrm{V}$ residuals before alignment.

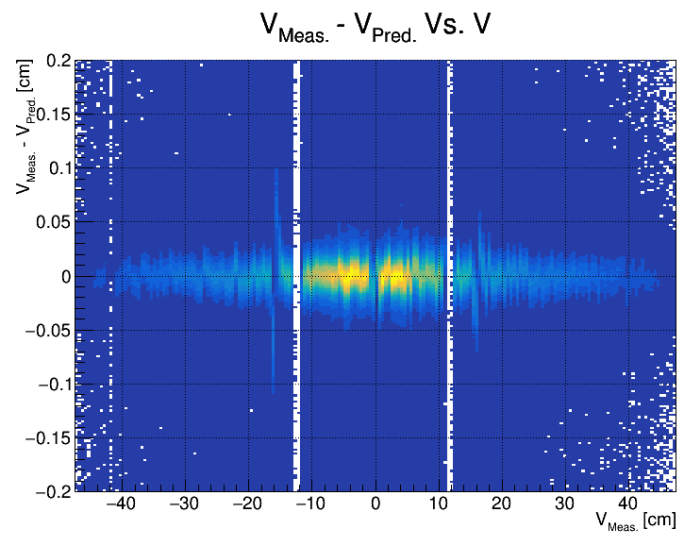

(d) V residuals after alignment.

Figure 3.10: Difference between measured cathode position and projected position using the opposite cathode hit for FDC plane 18 before and after internal alignment of the cathode pitch. 


\subsection{Track-Based Alignment}

The resolution of tracking detectors in the modern era typically meets or exceeds the resolution of survey measurements. In order to achieve design resolution in the tracking detectors, the detectors must be aligned to high precision. A variety of methods have been developed to determine this alignment using data from the detectors themselves. Three methods of alignment have been tested or applied in calibrations of the GlueX detector:

- Histogram based methods.

- Alignment with an extended Kalman filter.

- Alignment using closed-form methods (Millepede).

Histogram methods are the simplest of the alignment methods. In this method one simply plots the residuals along a track, then shifts according to the mean of a fit to the distribution. This method works fairly well for linear shifts of planar detectors, but is ill-suited to the general alignment problem and prone to local minima of the solution space. Alignment with an extended Kalman filter was also investigated in simulation, but never employed in calibrations of the GlueX data. The difficulty with this method is that it must be performed iteratively on a single CPU thread since the geometry must be updated after every track. A method that is easily parallelized is desirable. A closed form solution of the alignment problem with Millepede provides just such a method.

\subsection{Millepede}

\subsubsection{General Overview}

Millepede is a software program designed to provide an experiment-independent set of tools for detector alignment and calibrations. Millepede is a closed-form solution to the alignment problem. The alignment problem in question can be framed as a $\chi^{2}$ minimization problem as follows

$$
\chi^{2}=\frac{1}{2} \sum_{i}^{N} \frac{r_{i}^{2}}{\sigma_{i}^{2}}
$$

where $r_{i}$ is the residual of the $i$ th measurement defined as $r_{i}=m_{i}-f(\mathbf{x}) . m_{i}$ is the measured value, and $f(\mathbf{x})$ is the value predicted by the track model $f$ given a set of parameters $\mathbf{x}$. There is difficulty minimizing this form of the function since the dimensionality grows with increasing sample size. The problem then becomes numerically infeasible to minimize in reasonable time as it requires inversion of a very large matrix. In practice, the set of parameters that determine our predicted measurement are the alignment parameters and the track parameters of each fit. As the number of individual tracks included in the alignment sample increases, the number of track parameters grows with it. Therefore the problem as stated becomes numerically infeasible in reasonable time.

We can separate our parameters into two sets, local parameters that change from track to track, and global parameters that stay constant from one track to the next. The local parameters can be identified as the track parameters for each track, and the global parameters are the alignment parameters we are interested in. Millepede implements a bit of linear algebra magic that allows one to solve for the global parameters by themselves, eliminating the problem of dimensionality. The details of this method can be found in [33]. One of the benefits of Millepede is that the code that calculates the alignment parameters is rarely changed by the user. Instead, Millepede provides a robust user application programming interface (API) to provide input to these methods. 


\subsubsection{User API}

The Millepede program is actually broken into two separate steps. The Mille library is meant to write out a standardized file format containing all of the information needed for the alignment. This code is adapted by the user to collect the required information from their experiment-specific tracking software. Pede is a separate program used to perform the matrix inversion (approximate or exact) and return results for the alignment parameters. As an input to Mille, the user must provide the following:

1. The residual for each measurement on the track.

2. The uncertainty of this residual.

3. The derivative of the residual with respect to the track parameters.

4. The derivative of the residual with respect to the alignment parameters.

While simple in theory, access to these values within the GlueX software required considerable development.

It is perhaps easiest to understand the basic steps used in determining these values by formulating the simplest example. Imagine a straight track that has been fit through a hypothetical set of $\mathrm{N}$ planar detectors placed regularly in $\mathrm{z}$ that are only sensitive in the $\mathrm{x}$ direction. Parameterizing this track in $\mathrm{z}$, we may write

$$
x(z)=a+b z
$$

where $a$ is the value of $x(0)$ and $b$ is the slope $d x / d z$. After the fit to the track is completed, we have estimates of the two parameters in the fit, $a$, and $b$, and their covariance matrix $\mathbf{C}$. To project the residual from the $i$-th detector we write

$$
\begin{aligned}
r_{i} & =m_{i}-x\left(z_{i}\right) \\
& =m_{i}-\left(\begin{array}{ll}
1 & z_{i}
\end{array}\right)\left(\begin{array}{l}
a \\
b
\end{array}\right)
\end{aligned}
$$

where $m_{i}$ is the measurement on the $i$ th plane that went into the track fit. In general we may write

$$
\mathbf{r}=\mathbf{m}-\mathbf{H P}
$$

where $\mathbf{P}$ is a vector of the fit parameters and

$$
\mathbf{H}=\left.\frac{\partial x(z)}{\partial p_{j}}\right|_{z=z_{i}}
$$

is known as the projection matrix. The variance of the residual is then given as

$$
\sigma_{r_{i}}^{2}=\sigma_{m_{i}}^{2}-\left(\begin{array}{ll}
1 & z_{i}
\end{array}\right)\left(\begin{array}{cc}
\sigma_{a}^{2} & \sigma_{a b}^{2} \\
\sigma_{a b}^{2} & \sigma_{b}^{2}
\end{array}\right)\left(\begin{array}{c}
1 \\
z_{i}
\end{array}\right)
$$

where again in general we have the covariance matrix of the residuals $\mathbf{R}$ given as

$$
\mathbf{R}=\mathbf{V}-\mathbf{H C H}^{\top}
$$

where $\mathbf{V}$ is the measurement covariance matrix (typically diagonal) and $\mathbf{C}$ is the fit covariance matrix. The minus sign here is a result of the residual itself having already gone into the fit. Now imagine our detector is slightly misaligned in the direction to which it is sensitive, i.e.

$$
m_{i} \rightarrow m_{i}+\delta m_{i} .
$$


The residual is now

$$
r_{i}=m_{i}+\delta m_{i}-a-b z_{i} .
$$

We now have all of the pieces required as input to Millepede. The residual and the error on the residual are given by equations 3.19 and 3.23 . The derivatives with respect to the track parameters and the alignment parameters can be calculated from equation 3.26. These are

$$
\frac{\partial r_{i}}{\partial a}=-1, \frac{\partial r_{i}}{\partial b}=-z_{i}, \text { and } \frac{\partial r_{i}}{\partial \delta m_{i}}=+1 .
$$

This calculation is performed at each measurement along the track and the results are output in Mille format. This is repeated for a large sample of tracks each with their own set of track parameters. This result can then be processed with Pede to produce estimates for the alignment parameter itself. The alignment parameters can then be applied, and the process iterated until convergence is reached. In this case, since the measurement model is linear, the process will converge in a single iteration [33]. There is still one problem in that we have not yet fixed the global offset or slope of our detector system. Simultaneous shifts of all detector planes or displacements which are linear in $z$ will result in the same $\chi^{2}$ in equation 3.17. These are known as " $\chi^{2}$ invariant deformations" and must be constrained during the alignment procedure typically by fixing one of the elements in space.

\subsubsection{Millepede for GlueX}

The simple formulation presented in the previous section is in principle the same as the methods used for determining the input to Mille in GlueX, but the details are a bit more complicated. For a small taste of how this looks in the real experiment, let's lay the groundwork for another example, this time applied to the GlueX experiment. The key to using Millepede is deriving an expression for the residuals of each measurement. This time, imagine a straight track from a cosmic ray muon fit through the CDC. A straight line in three dimensions can be described by four parameters as a function of $\mathrm{z}$. We can parameterize the track as

$$
\begin{aligned}
& x(z)=x_{0}+t_{x} z \\
& y(z)=y_{0}+t_{y} z .
\end{aligned}
$$

As described earlier in Chapter 2, the CDC measures the drift distance from the wire. Thus, the residual we are interested in is the measurement minus the distance of closest approach of the track to the wire

$$
r_{i}=m_{i}-D O C A \text {. }
$$

This is now more difficult than our minimal example since the distance of closest approach is a nonlinear function of the track parameters.

The distance of closest approach can be calculated as follows. The wire position and direction are given by an origin location $\mathbf{w}_{0}=\left(w_{x}, w_{y}, w_{z}\right)$ and a vector along the direction of the wire $\mathbf{w}_{\text {dir }}=\left(w_{t_{x}}, w_{t_{y}}, w_{t_{z}}\right)$. We may write our track in a similar way by defining some point along the track as our origin $\mathbf{t}_{0}=(x, y, z)$ and the direction $\mathbf{t}_{\text {dir }}=\left(t_{x}, t_{y}, 1\right)$. Defining

$$
\begin{aligned}
\mathbf{d} & =\mathbf{t}_{0}-\mathbf{w}_{0} \\
D_{0} & =\left(\mathbf{t}_{d i r} \cdot \mathbf{t}_{d i r}\right)\left(\mathbf{w}_{d i r} \cdot \mathbf{w}_{d i r}\right)-\left(\mathbf{t}_{d i r} \cdot \mathbf{w}_{d i r}\right)^{2} \\
N_{0} & =\left(\mathbf{t}_{d i r} \cdot \mathbf{w}_{d i r}\right)\left(\mathbf{w}_{d i r} \cdot \mathbf{d}\right)-\left(\mathbf{w}_{d i r} \cdot \mathbf{w}_{d i r}\right)\left(\mathbf{t}_{d i r} \cdot \mathbf{d}\right) \\
N_{1} & =\left(\mathbf{t}_{d i r} \cdot \mathbf{t}_{d i r}\right)\left(\mathbf{w}_{d i r} \cdot \mathbf{d}\right)-\left(\mathbf{t}_{d i r} \cdot \mathbf{w}_{d i r}\right)\left(\mathbf{t}_{d i r} \cdot \mathbf{d}\right) \\
s_{0} & =\frac{N_{0}}{D_{0}} \\
s_{1} & =\frac{N_{1}}{D_{0}} .
\end{aligned}
$$


We may write the vector defining the length and direction of the line of closest approach as

$$
\mathbf{L O C A}=\mathbf{d}+s_{0} \mathbf{t}_{d i r}-s_{1} \mathbf{w}_{d i r} .
$$

The distance of closest approach is then the magnitude of this vector. Explicitly writing this out in terms of the track and wire parameters we have

$D O C A=\sqrt{\frac{\left(w_{t_{y}}\left(w_{x}-t_{x} w_{z}-x+t_{x} z\right)-\left(w_{t_{x}}-t_{x} w_{t_{z}}\left(w_{y}-y\right)+t_{y}\left(-w_{t_{z}} w_{x}+w_{t_{x}} w_{z}+w_{t_{z}} x-w_{t_{x}} z\right)\right)^{2}\right.}{\left(1+t_{y}^{2}\right) w_{t_{x}}^{2}+\left(1+t_{x}^{2}\right) w_{t_{y}}^{2}-2 t_{y} w_{t_{y}} w_{t_{z}}+\left(t_{x}^{2}+t_{y}^{2}\right) w_{t_{z}}^{2}-2 t_{x} w_{t_{x}}\left(t_{y} w_{t_{y}}+w_{t_{z}}\right)}}$

Now let's add some alignment parameters. In the experiment, we align the wires of the CDC by allowing the endpoints to shift in $\mathrm{x}$ and $\mathrm{y}$ while fixed in $\mathrm{z}$. We label these parameters $\delta x_{u}, \delta y_{u}, \delta x_{d}$ and $\delta y_{d}$ where the subscripts refer to the upstream and downstream ends of the wire. This changes our definition of the wire position and direction as follows

$$
\begin{aligned}
\mathbf{w}_{0} & =\left(w_{x}, w_{y}, w_{z}\right) \\
& =\left(\frac{x_{d}+x_{u}}{2}, \frac{y_{d}+y_{u}}{2}, \frac{z_{d}+z_{u}}{2}\right) \\
& \rightarrow\left(\frac{\left(x_{d}+\delta x_{d}\right)+\left(x_{u}+\delta x_{u}\right)}{2}, \frac{\left(y_{d}+\delta y_{d}\right)+\left(y_{u}+\delta y_{u}\right)}{2}, \frac{z_{d}+z_{u}}{2}\right)
\end{aligned}
$$

and

$$
\begin{aligned}
\mathbf{w}_{d i r} & =\left(w_{t x}, w_{t y}, w_{t z}\right) \\
& =\left(x_{d}-x_{u}, y_{d}-y_{u}, z_{d}-z_{u}\right) \\
& \rightarrow\left(\left(x_{d}+\delta x_{d}\right)-\left(x_{u}+\delta x_{u}\right),\left(y_{d}+\delta y_{d}\right)-\left(y_{u}+\delta y_{u}\right), z_{d}-z_{u}\right)
\end{aligned}
$$

These replacements can then used in the calculation of equation 3.29. As can be seen, the derivatives we need of equation 3.28 with respect to the track and alignment parameters are no longer as simple as in our initial example. In practice, these have been calculated by Mathematica [34] for use in the code.

In the case of curved tracks in a magnetic field, the situation becomes even more complicated. There are two different parameterizations of the track in the experiment depending on the polar angle. Each of these has five parameters to describe the helical shape of the particles in the magnetic field. In these cases, we no longer compute many of the derivatives analytically. These are instead calculated numerically by recalculating the residuals for small shifts in the track parameters. The derivatives have been implemented in the GlueX codebase and are now accessible as part of the standard fitting routines. Tools for Mille output from straight and curved tracking have also been released with the main analysis software package sim-recon [35]. A table describing the various alignment parameters implemented in the code can be found in Table 3.1.

This input to Mille uses results obtained through the standard GlueX track fitter using an extended Kalman filter [36]. A Kalman filter is not ideally suited as input to the method since the full covariance $\mathbf{C}$ in equation 3.24 is not calculated. Instead there is only a block-diagonal form of $\mathbf{C}$ returned missing the off-diagonal elements. This is fine as long as the measurements themselves are uncorrelated. However, the inclusion of energy loss and multiple scattering does correlate the residuals in the case of tracks measured in a magnetic field. Without the magnetic field on, the momentum of the particle is not known, so these processes are not included. This results in a slightly incorrect value of the error on the residual provided to Millepede for field-on measurements. This can lead to small biases in the returned alignment parameters. These are plans to include these correlations in the alignment procedure as outlined in Reference [37]. For the first pass at the global 
CDC

\begin{tabular}{|c|c|c|}
\hline \hline Alignment Parameter & Total Number of Parameters & Notes \\
\hline Up and downstream $\delta x$ and $\delta y$ & 14,088 & \\
Global translation and rotation & 6 & \\
Wire $t_{0}$ & 3,522 & \\
\hline
\end{tabular}

FDC

\begin{tabular}{|c|c|c|}
\hline \hline Alignment Parameter & Total Number of Parameters & Notes \\
\hline Cathode Alignment & 96 & \\
Strip Pitch & 240 & Five values per cathode plane \\
Cell position offset & 72 & \\
Cell rotation & 72 & Implemented but not tested \\
Cathode strip gain & 10,368 & Implemented but not tested \\
Wire $t_{0}$ & 2,304 &
\end{tabular}

Table 3.1: Table of alignment parameters implemented in the GlueX codebase.

\begin{tabular}{ccc} 
Run Number & Magnetic Field & Comments \\
\hline 10181 & $0 \mathrm{~A}$ & Cosmic run, BCAL trigger \\
11055 & $0 \mathrm{~A}$ & $40 \mathrm{nA} e^{-}$beam, $1 \times 10^{4}$ RL Al radiator \\
11529 & $1200 \mathrm{~A}$ & $150 \mathrm{nA}$ beam, Diamond radiator
\end{tabular}

Table 3.2: Runs used for alignment of the CDC and FDC in the Version 4 reconstruction.

tracking alignment, this effect has been ignored as it leads to small effects relative to our initial misalignments. The correct errors will be useful in the future when methods constraining alignment through tracks with a common vertex or known mass are used to improve the alignment results [38].

\subsubsection{Millepede Alignment Procedure}

Described in this section are the steps performed to determine the alignment parameters for the data presented in this thesis. This process will continue to evolve in future analyses as additional inputs to the alignment procedure are developed such as vertex and mass-constrained combinations of tracks. To start the procedure, all of the values of the alignment parameters were set to reflect nominal alignment of the detector. Position and relative timing offsets were set to zero, and multiplicative constants like the strip pitch in the FDC cathodes were set to the nominal value. The first step was to align the cathodes and wires in the FDC internally. This procedure was performed as described in Section 3.2. The next step was performed using track-based alignment. A group of three runs was processed to produce Mille files that were used in each alignment step. A sample of approximately 5 million triggers per run was used. A list of the runs used in this calibration can be found in Table 3.2 .

The first iterations of the track-based alignment adjusted the following calibrations:

1. The CDC wire offsets were adjusted in the $x$ and $y$ direction at the upstream and downstream end of each wire. The upstream and downstream endpoint position was assumed to be fixed in the $z$ direction along the beam.

2. The FDC wire planes were allowed to rotate about the beam axis, and each wire plane was allowed to shift perpendicular to the wire direction. The position in $z$ was fixed.

3. The FDC cathode planes were allowed to rotate about the beam axis, and each plane was allowed to shift along the wire direction. The position in $z$ was fixed. 
4. The pitch values for the FDC cathodes were allowed to change, while the alignment of wires and cathode planes was maintained using constraints in the determination of the alignment constants.

The following set of constraints were applied to the results from Pede in order to avoid some undesirable $\chi^{2}$ invariant deformations in the CDC:

$$
\begin{aligned}
\sum_{i=1}^{N_{\text {straws/ring }}}\left(\delta x_{u, d}\right)_{i} \sin \phi_{i} & =0 \\
\sum_{i=1}^{N_{\text {straws/ring }}}\left(\delta x_{u, d}\right)_{i} \cos \phi_{i} & =0
\end{aligned}
$$$$
\begin{aligned}
\sum_{i=1}^{N_{\text {straws/ring }}}\left(\delta y_{u, d}\right)_{i} \sin \phi_{i} & =0 \\
\sum_{i=1}^{N_{\text {straws/ring }}}\left(\delta y_{u, d}\right)_{i} \cos \phi_{i} & =0
\end{aligned}
$$

where $\phi_{i}$ is the phi angle of the $i$-th wire endpoint in lab coordinates and $\delta x_{u}, \delta y_{u}, \delta x_{d}$ and $\delta y_{d}$ are the alignment constants describing $x$ and $y$ shifts at the upstream and downstream end of each wire. This constraint was applied separately in each of the 28 rings of the CDC $(4 \times 28$ constraint equations). The effect of this constraint is to force the total projection of the alignment parameters onto the radial direction in each ring to be zero. Hence, the CDC can not stretch or shrink in the radial direction as a result of the alignment.

In order to maintain the alignment of the FDC wire and cathode planes determined in the internal alignment step, the following constraint equations were used

$$
\begin{aligned}
& \sin \phi_{v} \delta p_{u}+\sin \phi_{u} \delta p_{v}-\frac{\left(p_{v}+p_{u} \cos \left(\phi_{u}-\phi_{v}\right)\right) \sin \phi_{v}}{\sin \left(\phi_{u}-\phi_{v}\right)} \delta \phi_{u}+\frac{\left(p_{u}+p_{v} \cos \left(\phi_{u}-\phi_{v}\right)\right) \sin \phi_{u}}{\sin \left(\phi_{u}-\phi_{v}\right)} \delta \phi_{v}=0 \\
& \sin \phi_{v} \delta p_{u}-\sin \phi_{u} \delta p_{v}+\frac{\left(p_{v}-p_{u} \cos \left(\phi_{u}-\phi_{v}\right)\right) \sin \phi_{v}}{\sin \left(\phi_{u}-\phi_{v}\right)} \delta \phi_{u}+\frac{\left(p_{u}-p_{v} \cos \left(\phi_{u}-\phi_{v}\right)\right) \sin \phi_{u}}{\sin \left(\phi_{u}-\phi_{v}\right)} \delta \phi_{v}=0
\end{aligned}
$$

where $\phi_{u}$ and $\phi_{v}$ are the aligned angles as in Figure 3.7, and $p_{u}$ and $p_{v}$ are the average strip pitch on each cathode plane, and $\delta \phi_{u}, \delta \phi_{v}, \delta p_{u}$, and $\delta p_{v}$ are the alignment parameters describing shifts to these parameters. This constraint was applied separately to each of the 24 FDC planes. These fix the FDC wire/cathode alignment along the lines $u=v$ and $u=-v$ which in turn fixes the alignment across the plane.

Convergence for this step typically takes 3-5 iterations where the new alignment constants are applied at each step. Once this procedure converged, the final step in the per-channel CDC alignment was adjusting the $t_{0}$ of each wire while keeping the endpoint offsets fixed. After this step, the CDC per-channel calibration was fixed.

The final step in the alignment was to allow global rotations of the CDC relative to the FDC packages. In this step, the FDC remains fixed, while the CDC is allowed to shift in position and orientation. This was alternated with a second procedure where the CDC was fixed and the individual FDC packages were allowed to shift. Here the results were constrained such that the total offset of the each package of six planes is zero. After inspecting the residuals at the end of the full procedure, small additional alignment steps were performed, but were not found to greatly improve the quality of the reconstruction.

\subsubsection{Results using Millepede for Alignment of GlueX}

The raw data for the Spring 2016 run period takes roughly 30 CPU years to process in its entirety, so full reconstructions of the data are performed sparingly. There have so far been four reconstructions 
of the this data set. The early reconstruction passes were suitable for measurements of asymmetries between the PARA and PERP polarization orientations. In these measurements, the experimental acceptance cancels in the calculation of the observables. For an analysis requiring acceptance corrections, it is necessary to validate that the cuts performed in the analysis behave similarly in data and Monte Carlo across a wide range of kinematics. Version 3 of the reconstruction suffered from some inconsistencies in the charged tracking when studying these effects. It was noted that tight cuts on the kinematic-fit confidence level would skew the $\phi$ distribution of the accepted charged tracks, which could be an indication that either the reconstructed values were incorrect or the errors assigned to the particle kinematics were inconsistent with the measurement.

A very likely cause of these effects was misalignments of the tracking detectors. There had been some effort already within the collaboration to align the tracking detectors, but these efforts were split between the two different detectors and tools for proper validation of the fit results and calibrations did not exist. The tools for validation were deployed with the version-3 reconstruction. Plotting the mean of the residual distribution as a function of the position on the FDC detector provides a way to visualize the correlations in misalignments in each detector plane. Each FDC hit contributes a 2D measurement to the fit. The cathodes are used to measure the direction along the wire, and the drift time from the wires are used to measure the perpendicular direction to the wire. In the plots that follow, each plane is oriented such that the wires are always along the y-axis. These are the local coordinates of the FDC package. Keep in mind that in lab coordinates, each FDC plane is rotated relative to the previous by $60^{\circ}$. The results for the average residuals from curved tracks in the FDC from the version-3 reconstruction are shown in Figures 3.11 and 3.12. These results show clear correlations in some the planes that can be indicative of various forms of misalignment. These misalignments can cause the reconstructed particle momenta to be incorrectly calculated. 

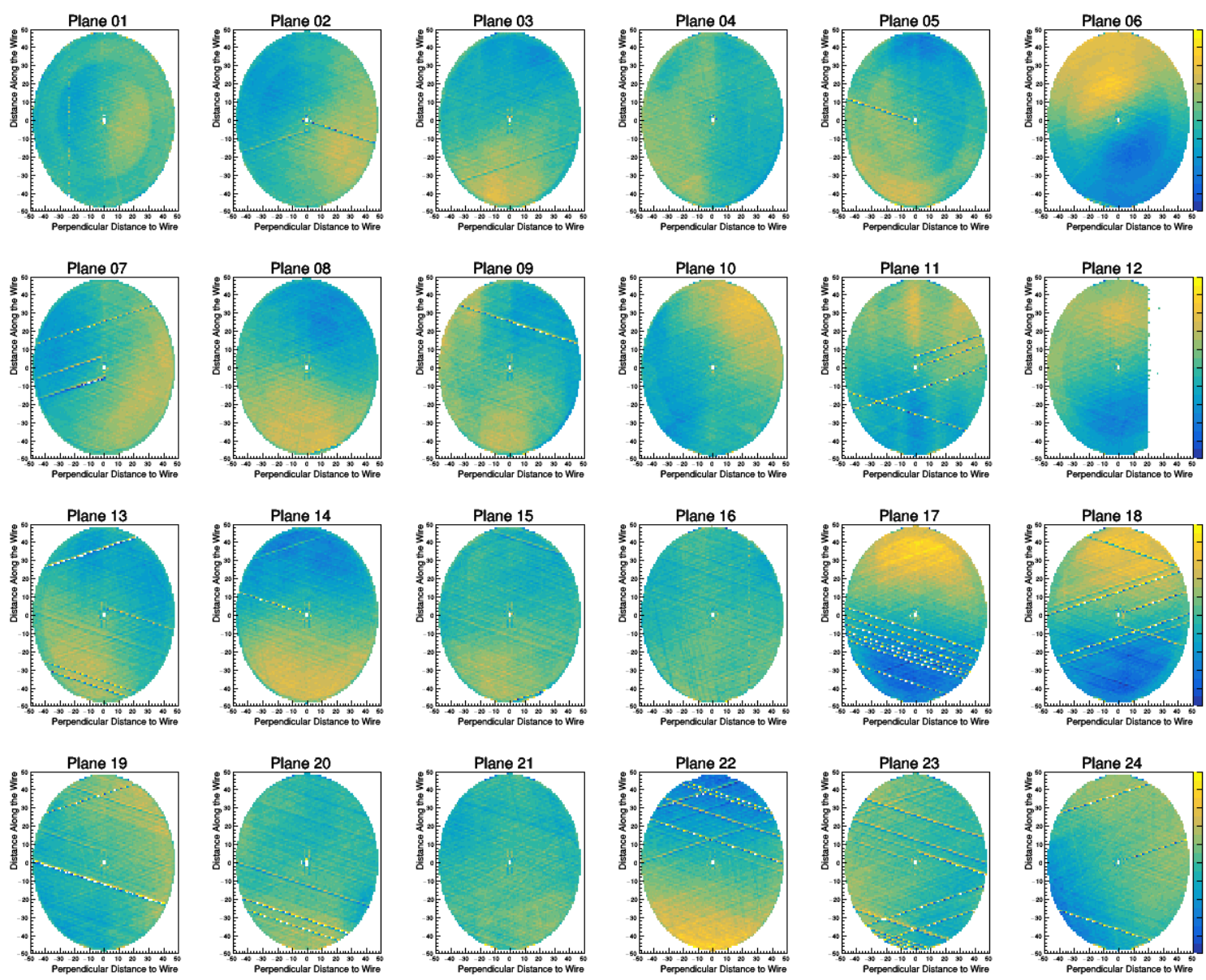

Figure 3.11: Average FDC cathode residual versus local xy position for each FDC package in the Version 3 reconstruction. The residuals are plotted on a scale of $\pm 250 \mu \mathrm{m}$. 

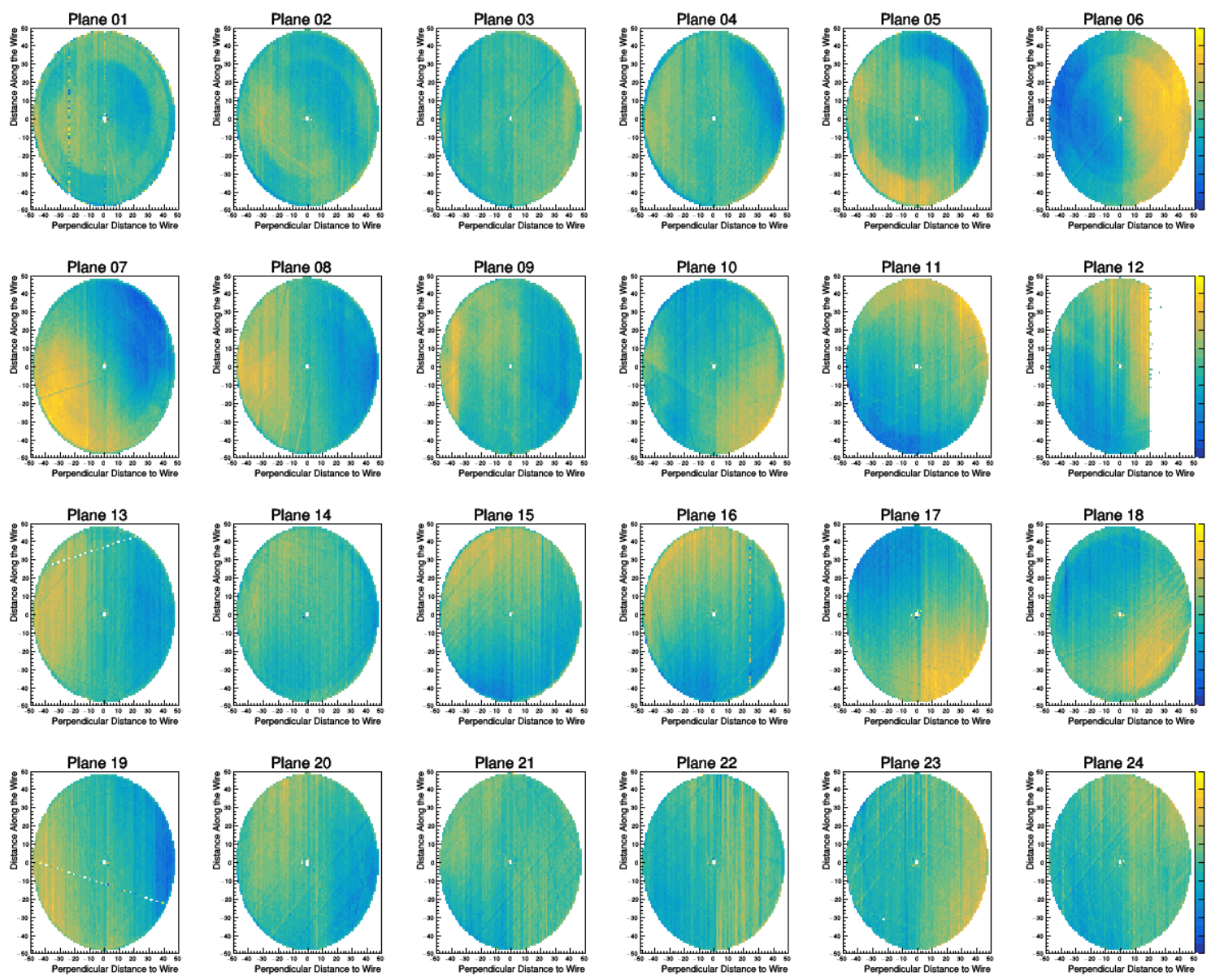

Figure 3.12: Average FDC wire residual versus local xy position for each FDC package in the version 3 reconstruction. The residuals are plotted on a scale of $\pm 250 \mu \mathrm{m}$. 
Millepede was used to attempt to correct for these effects in the reconstruction. The results after using the procedure described in Section 3.4.4 are shown in Figures 3.13 and 3.14. Here the results are greatly improved and many of the correlations seen in the version-3 reconstruction have been corrected. There is still some residual misalignment in the FDC wire measurement that is not yet entirely understood. This is a topic of continued investigation within the collaboration.

Notably absent from our discussion so far are comparisons for the CDC. For reconstruction version 3 we do not have the necessary plots for comparison due to a bug that was later fixed in the tracking code. Instead we present here the result of aligning the CDC from scratch using Millepede. In Figure 3.15 we present the residuals for each straw in the CDC where the straw spatial and timing offsets of the individual wires have been set to zero. Figure 3.16 shows the result after aligning these two sets of constants using Millepede. The uniformity from straw to straw in the detector improves dramatically through the alignment. 

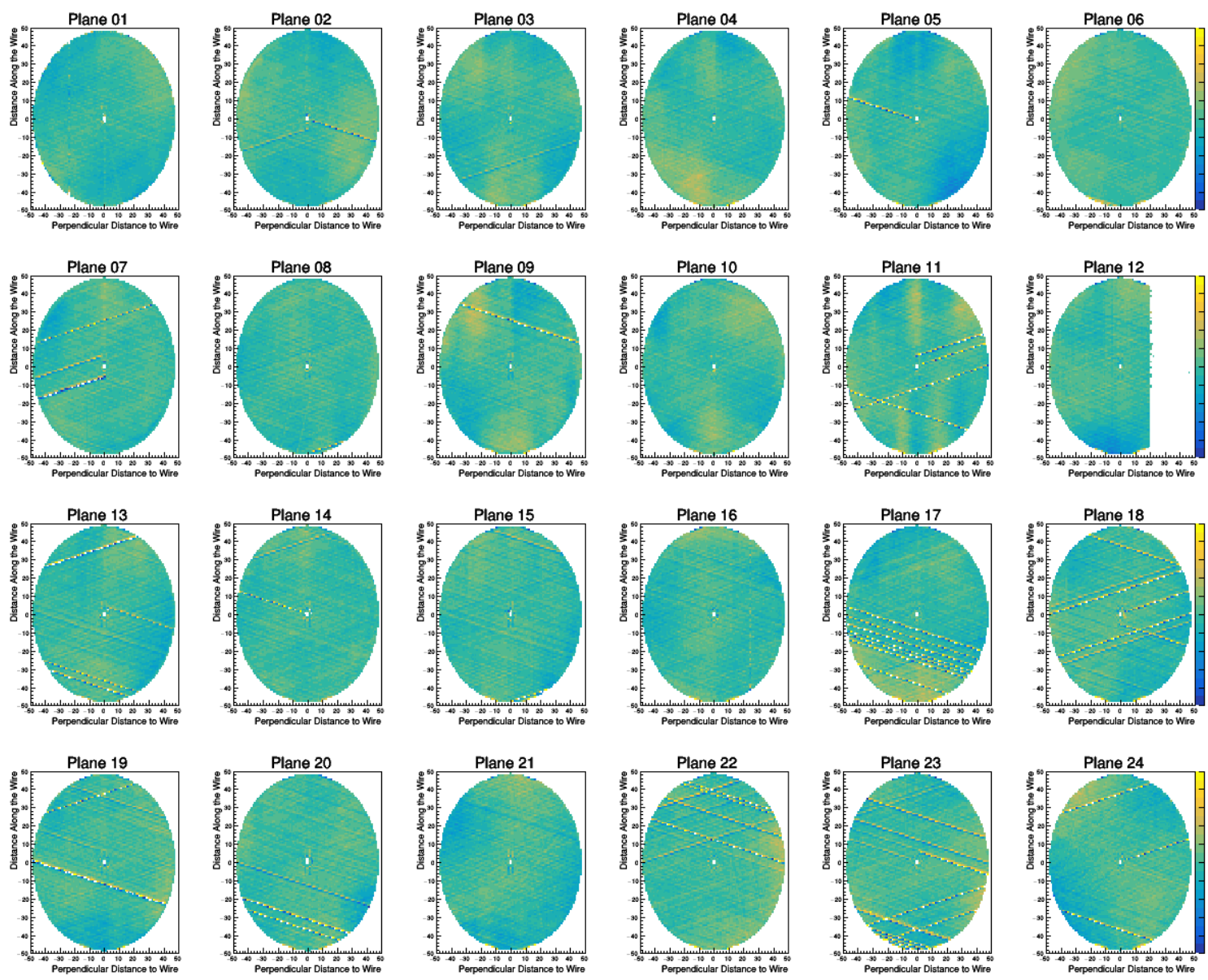

Figure 3.13: Average FDC cathode residual versus xy position for each FDC package after the Millepede alignment procedure. The residuals are plotted on a scale of $\pm 250 \mu \mathrm{m}$. 

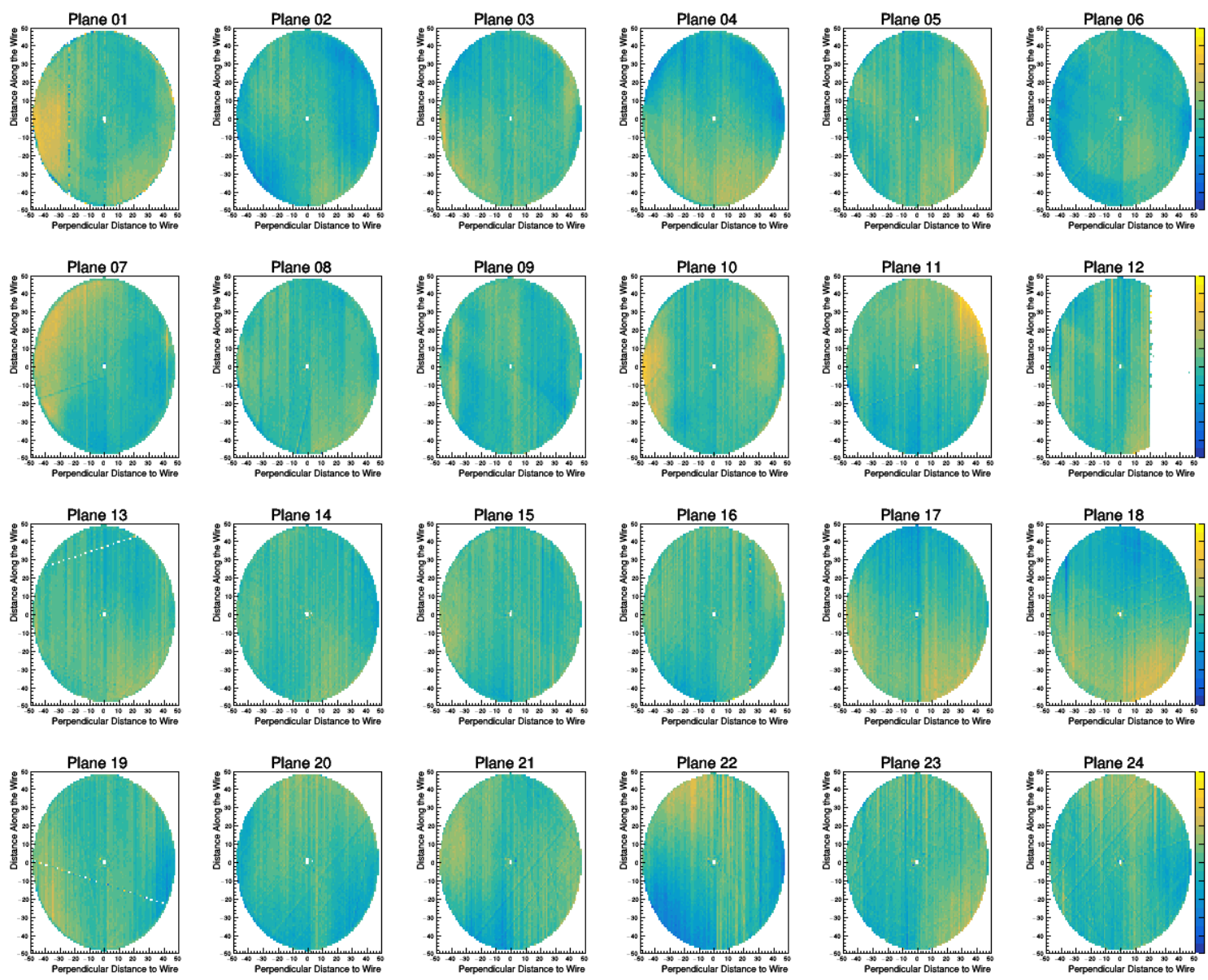

Figure 3.14: Average FDC wire residual versus xy position for each FDC package after the Millepede alignment procedure. The residuals are plotted on a scale of $\pm 250 \mu \mathrm{m}$. 

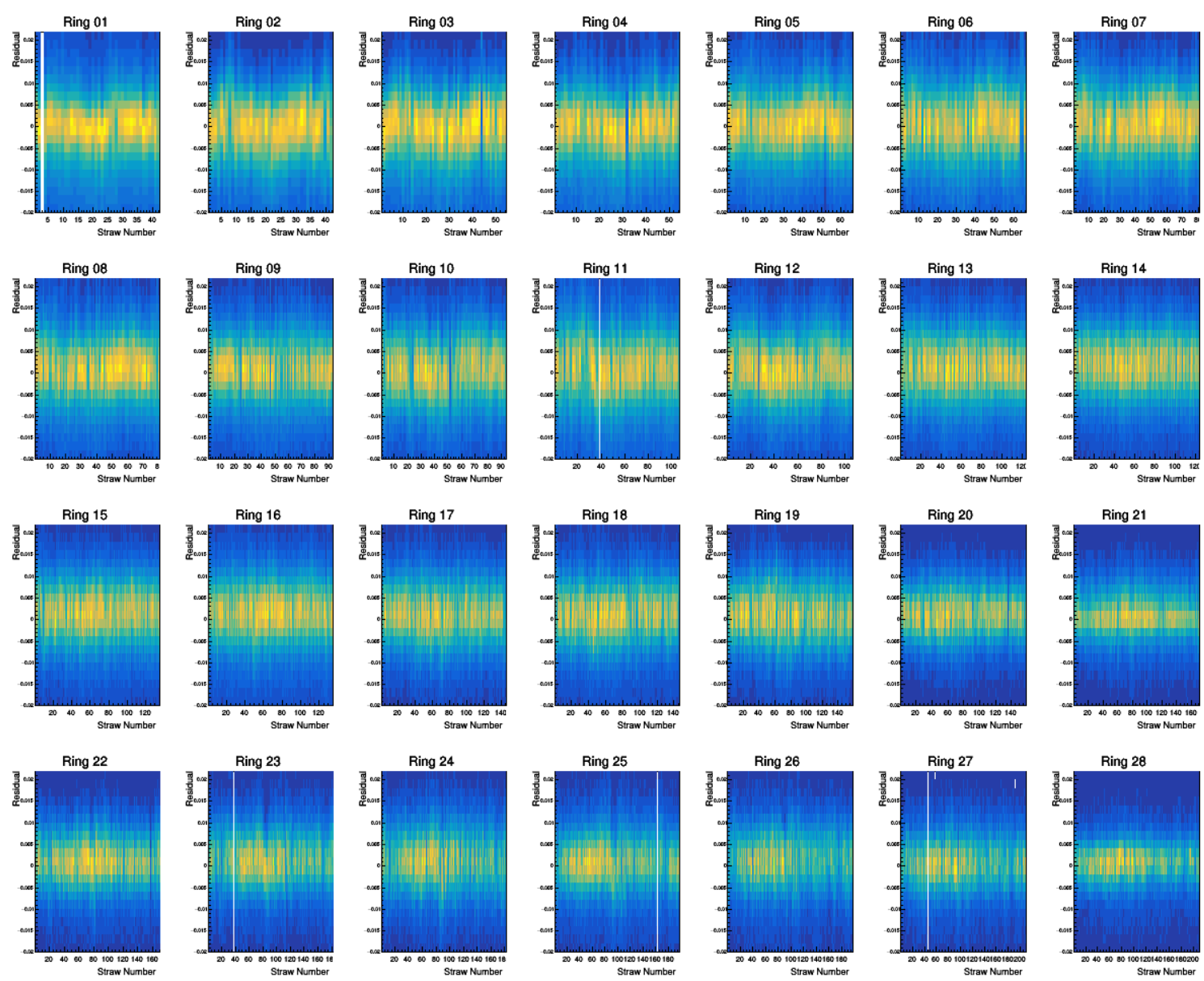

Figure 3.15: Residuals for hits on all straws in the CDC before alignment. Each plot represents one ring of the $\mathrm{CDC}$. The $\mathrm{x}$-axis is the straw number in a given ring, and the $\mathrm{y}$-axis is the residual from tracking on a scale of $\pm 200 \mu \mathrm{m}$. 

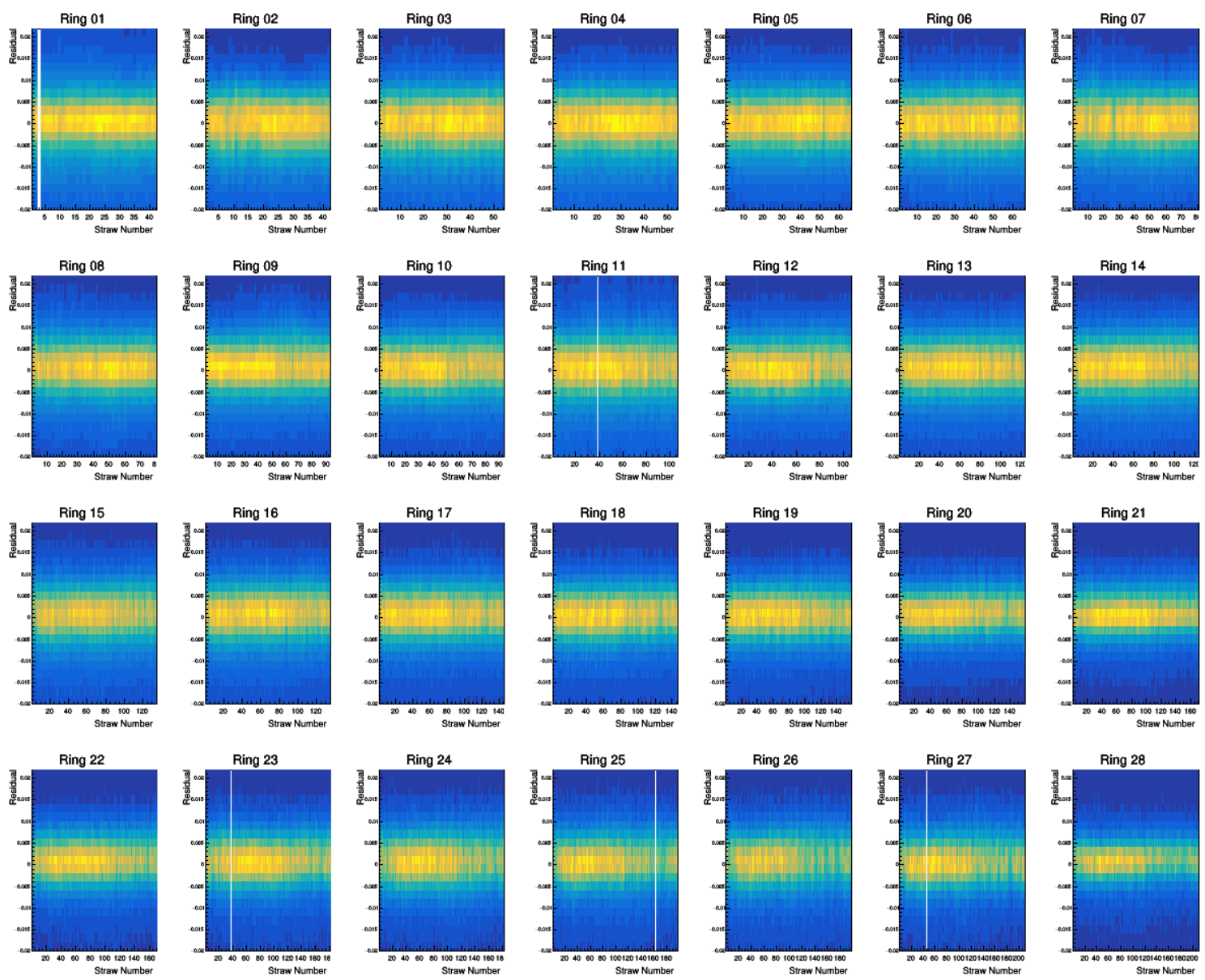

Figure 3.16: Residuals for hits on all straws in the CDC after alignment with Millepede. The upstream and downstream positions of the wires have been aligned, as has the timing offset for each wire. Each plot represents one ring of the CDC. The $\mathrm{x}$-axis is the straw number in a given ring, and the $\mathrm{y}$-axis is the residual from tracking on a scale of $\pm 200 \mu \mathrm{m}$. 


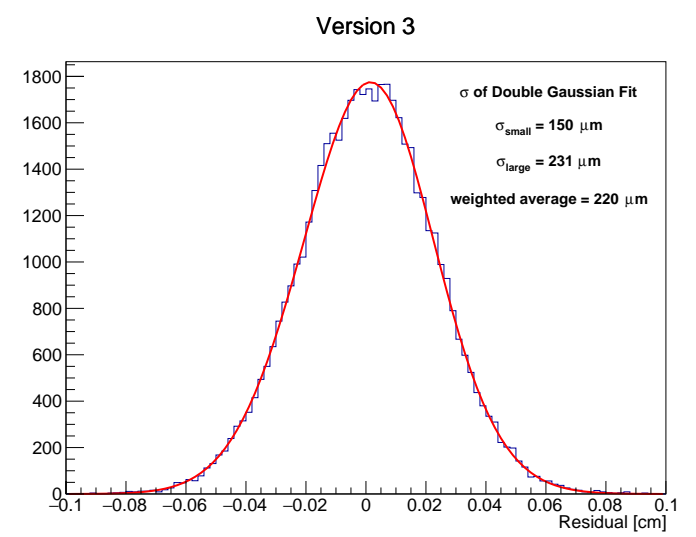

(a)

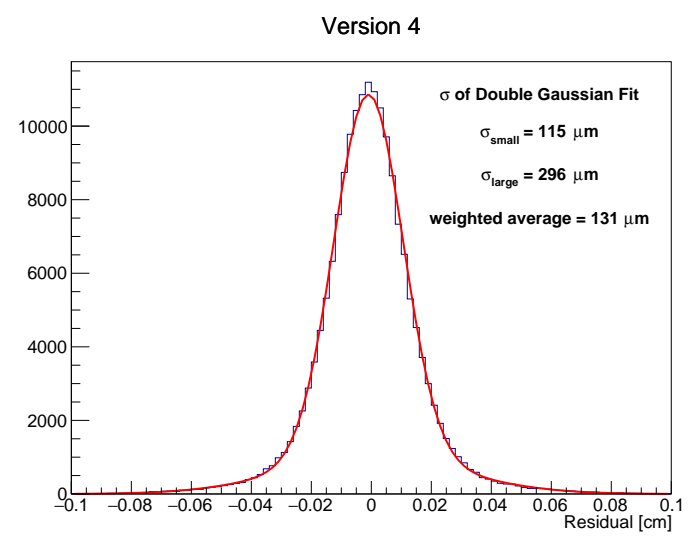

(b)

Figure 3.17: Unbiased residuals in the direction parallel to the wire for plane 6 of the FDC for straight tracks in run 11055. Results are presented for (a) version 3 constants and (b) version 4 constants.

To quantify our result, we may look at the residual distributions from the detectors before and after calibration. Due to the multiple-scattering and energy-loss modeled in the field-on fits, the residual distributions become slightly distorted from the process noise added during the fit. The distributions for straight tracks tend to be easier to fit to extract the resolutions of the detectors. We compare unbiased (the plane is not included in the fit) residuals for plane 6 of the FDC for the alignment constants used in the version-3 and 4 reconstruction of the Spring 2016 data in Figures 3.17 and 3.18. The biased CDC residuals are compared before and after Millepede alignment in Figure 3.19. Tracks are selected with at least 15 hits for the plots that follow, so bias should be minimal. The residual distributions are fit with the sum of two Gaussians, one to describe the central peak, and another to describe the wide tails. The width of these distributions are referred to as $\sigma_{\text {small }}$ and $\sigma_{\text {large. }}$. The weight used to determine the average $\sigma$ is given by the ratio of the integral of each Gaussian to the total integral. These fits indicate an improvement of the FDC residuals along the wire from 220 to $131 \mu \mathrm{m}$ between the version- 3 and version- 4 reconstruction. The residuals perpendicular to the FDC wires decrease from 240 to $174 \mu \mathrm{m}$. Both of these values are better than the design spatial resolution of the FDC which is $200 \mu \mathrm{m}$. In the CDC, alignment with tracks shrinks the residual from 150 to $130 \mu \mathrm{m}$. The design resolution of the CDC is $150 \mu \mathrm{m}$. These calibrations were clearly needed in order to beat the design resolutions of the detectors.

Another feature to consider when evaluating the quality of the charged-track reconstruction is inspecting the kinematic-fit confidence level distribution in a fit to a fully charged final state. We performed a kinematic fit to the hypothesis of conserved four-momentum and shared vertex location for the reaction $\gamma p \rightarrow p \pi^{+} \pi^{-}$. In Figure 3.20 is plotted the confidence level of the fit (see Section 4.3.2) for the version-3 and 4 reconstructions. Suffice it to say at this point that "flatter is better". In these plots we have not applied any other selection cuts or accidental subtraction as is the case in the later Figure 4.3. Clearly there is improvement from version-3 to version- 4 . 


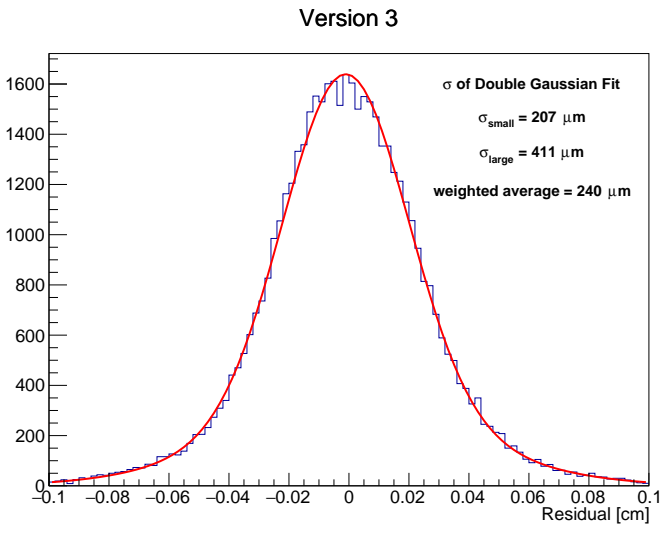

(a)

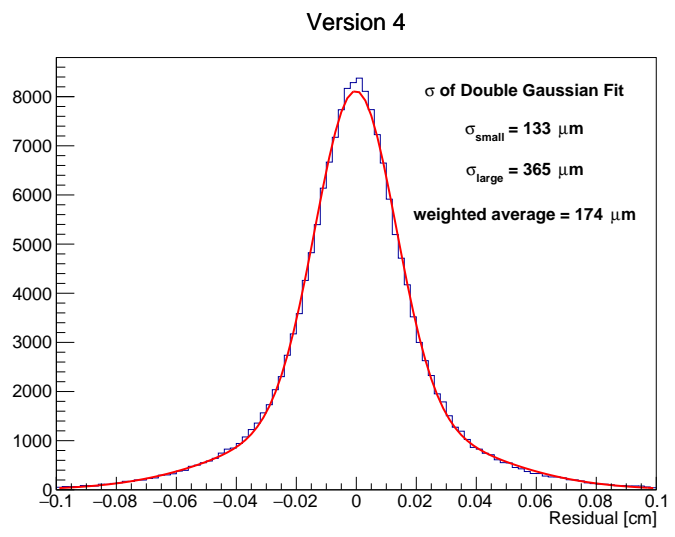

(b)

Figure 3.18: Unbiased residuals in the direction perpendicular to the wire for plane 6 of the FDC for straight tracks in run 11055. Results are presented for (a) version 3 constants and (b) version 4 constants.

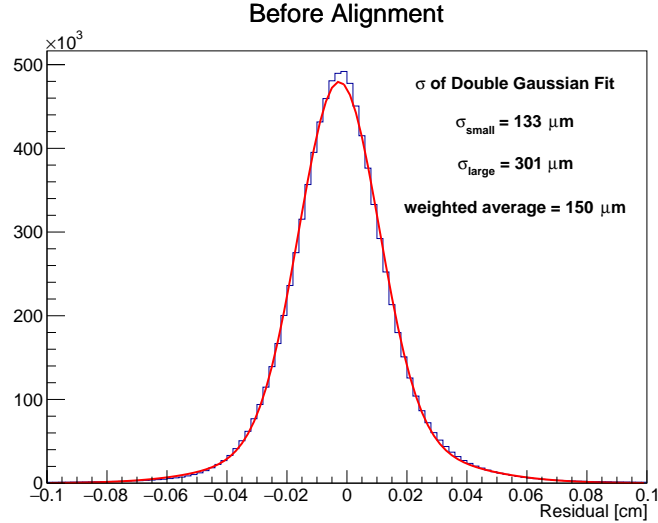

(a)

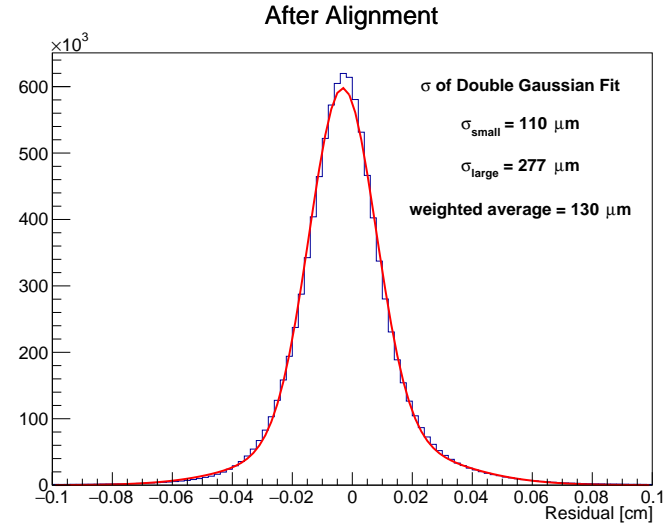

(b)

Figure 3.19: Biased residuals for straight tracks in all wires of the CDC in run 10181. Results are presented (a) before and (b) after alignment of the wire positions and timing offsets with Millepede. $t_{d r i f t}<100 \mathrm{~ns}$ is excluded from this plot. A fit to a double Gaussian is performed. 


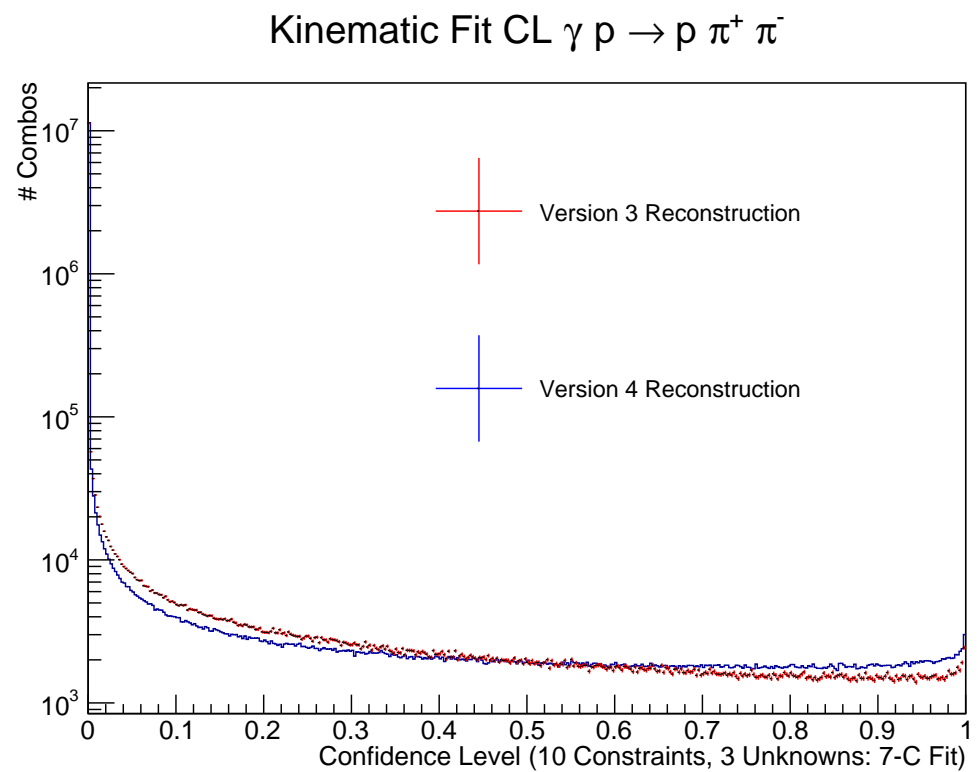

Figure 3.20: Kinematic fit CL for $\gamma p \rightarrow p \pi^{+} \pi^{-}$in version 3 and 4 REST production. Version 4 is closer to the ideal distribution (see Section 4.3.2). 


\section{Chapter 4}

\section{Event Selection}

\subsection{Data Selection}

In this chapter we describe the selection of data that will be used in Chapter 5 to extract the spin density matrix elements in $\omega$ photoproduction. The data were collected from April 14-20, 2016 at the tail end of the final GlueX engineering run period. While much of this run period was spent studying hardware performance and tweaking the experimental parameters, the set of runs from 11366 to 11555 was a period of stable "production" running during which minimal changes to the experiment were made. This set of runs has come to be known colloquially as the golden run period. A summary of the event statistics in the golden run period is presented in Table 4.1.

During this set of runs, the polarized photon beam was produced on a $50 \mu \mathrm{m}$ thick diamond radiator and passed through the $3.4 \mathrm{~mm}$ collimator. The diamond was rotated between two perpendicular orientations, PARA where the polarization vector is parallel to the floor, and PERP where the polarization vector is perpendicular to the floor. These two orientations are considered as separate independent data sets through most of the analysis. The results are only recombined to get our final result and to produce the plots in this chapter.

The electron beam was delivered at $250 \mathrm{MHz}$ from the accelerator, corresponding to a bunch spacing of roughly $4 \mathrm{~ns}$. The GlueX solenoid was set to a current of $1200 \mathrm{~A}$ which is slightly lower than nominal operation in subsequent run periods. This slightly impacts the momentum resolution for charged tracks, but simulations indicate much of the resolution that is lost can be recovered for exclusive reconstruction by kinematically fitting the event [39].

During the golden run period, the nominal beam current had to be set slightly higher for PERP (138 nA) than PARA (128 nA) configurations in order to match DAQ rates in the hall between the two settings. This has been attributed to possible misalignment of the photon beam spot on the collimator. Efforts to understand the impact of the backgrounds caused by this increased beam current are still ongoing. For this analysis, it is assumed that the subtraction of tagger accidentals described in section 4.3.1 removes the dependence of the results on the beam current.

\begin{tabular}{|c|c|c|c|}
\hline Radiator & Polarization Orientation & Number of Triggers & Average $e^{-}$Beam Current \\
\hline \hline $50 \mu \mathrm{m}$ diamond & PARA & $2,680,858,358$ & $128 \mathrm{nA}$ \\
\hline $50 \mu \mathrm{m}$ diamond & PERP & $2,878,849,718$ & $138 \mathrm{nA}$ \\
\hline $3 \times 10^{-5} \mathrm{RL} \mathrm{Al}$ & Amorphous & $825,150,336$ & $129 \mathrm{nA}$ \\
\hline
\end{tabular}

Table 4.1: Summary of total statistics and run conditions in the Spring 2016 golden run period. 


\subsection{Data Processing}

There are several stages in our analysis chain during which cuts are applied to the data to extract our signal. After calibration, the raw data is processed into a compressed XML data format for Reconstructed Event STorage (REST). Reconstructed momentum/energy four-vectors, positions of the tracks and showers and their covariance matrices are stored. All hit-level information is discarded. When improvements to the low-level calibrations or reconstruction are made, these REST files must be reproduced from the raw data. The analysis presented in this thesis was performed with the version 4 reconstruction of the Spring 2016 data. This REST production includes the improvements made to the tracking detector alignment and calibrations described in chapter 3 .

\subsection{Assembly of Particle Combinations}

Starting from the REST data, we can form combinations of tracks and showers consistent with our desired final states. For the hadronic decay, combinations consistent with two positively charged tracks, one negatively charged track and two neutral showers are assembled from the reconstructed data. Both mass hypotheses are tested for the proton and $\pi^{+}$in the case of the positive tracks $\left(p_{1} \pi_{2}^{+}\right.$and $\left.\pi_{1}^{+} p_{2}\right)$. In the case of the radiative decay, one positively charged track assigned to the mass of the proton and three neutral showers are required. Up to four additional "good" charged tracks besides those used in the combination are permitted in the event. These "good" tracks are simply those that have a matching hit in one of the detectors besides the tracking chambers. There are no other cuts on the track quality. We also require that there are no extra neutral showers in the calorimeters. The significance of this cut is currently being investigated. Results may change as improvements are made to charged-track clustering in the BCAL.

\subsubsection{Beam Photon Selection}

As part of the track-fitting routines, charged tracks are matched to hits in the SC, TOF, BCAL, and FCAL. Using the path-length of the track, the time of the matched hit is propagated to the point of closest approach to the beamline. This is the "vertex time". In the GlueX experiment, the reference plane for timing is chosen to be at the center of the liquid-hydrogen target. A correction is made to the vertex time to account for the distance between the vertex location and our reference plane. We will refer to the time at the center of the target as the particle's "target time", $t_{\text {Target }}$. In order to match the combination to a tagged beam photon, each of the charged particles in the combination vote by selecting the nearest 4 ns beam bucket to each $t_{\text {Target }}$ for every matched hit. Photons detected in the calorimeters are given a single vote. Photon flight times are corrected based on the position of the photon shower. Each vote is given equal weight. Ties are then broken by the summed squared residual of each target time to the RF signal coming from the accelerator. The "RF Time" is then reported as the incoming beam photon time at the center of the target for this selected beam bucket.

The selected RF time is then compared to the time of the reconstructed tagged photons. The tagger is calibrated such that each of the individual channels are aligned to the same RF signal. A cut is placed on the difference between the measured time in the tagger and the selected RF time in the main spectrometer. In most cases, the correct beam photon associated with the event should appear centered near $\Delta t=0$ if our selection procedure is successful. A plot of the difference between the tagged photon time and the RF time selected by the main spectrometer is shown in figure 4.1. In addition to this main peak, there are clearly additional bunches to the side spaced at the period of the beam bunches. These "accidental tags" are typically caused by real electron hits in other tagger channels near the photon energy that caused the trigger. Occasionally, the accidental hit will happen to arrive at the same time as the correct photon in a nearby counter. The structure seen in the sidebands is therefore presumably lurking under the main peak. We can correct for this 


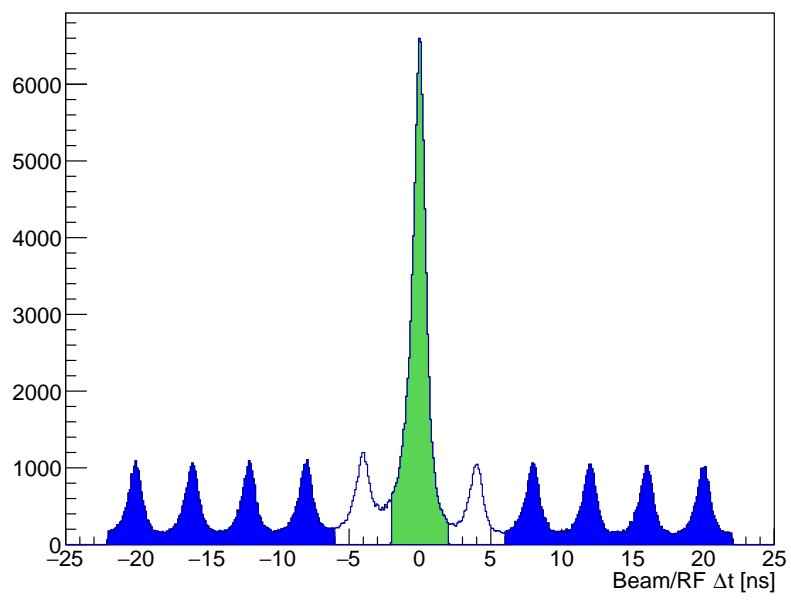

Figure 4.1: Difference between hit time in the tagger and the selected RF bunch in the main spectrometer. The sideband bunches (blue) are used to subtract the contribution of tagger accidentals from the main peak (green).

"accidental background" by subtracting the contribution from the sidebands in our analysis. It will be indicated as "accidentals-subtracted" in the caption of figures to follow when this has been done.

It is clear from figure 4.1 that the individual beam bunches are not resolved perfectly. The width of these distributions is driven almost entirely by the timing resolution of the tagger microscope. In the Spring 2016 run period, there were many individual fibers that did not meet the original design specification. These fibers had low light yield causing poor timing resolution. This poor resolution causes some of the spill over from the main peak into the adjacent bunches. We have chosen to cut these directly adjacent bunches from the analysis. Most of these low-yield fibers were replaced before the Spring 2017 run period.

\subsubsection{Kinematic Fitting}

A kinematic fit attempts to optimize measured particle parameters by constraining the results to match a hypothesis about the underlying physics process. In this analysis, the particle combination along with its beam photon are subjected to a kinematic fit to the hypothesis of conserved fourmomentum. In the hadronic decay, $\omega \rightarrow \pi^{+} \pi^{-} \pi^{0}$, the hypothesis of a common vertex is also included in the fit. In the kinematic fit, the mass of the $\pi^{0}$ originating from the decay of the $\omega$ is constrained to the PDG value in the fit as the width is negligible. However, the mass of the $\omega$ meson is not fixed, as it has non-negligible width. We define $\boldsymbol{\eta}$ as the estimated observable parameters (momenta and position), $\mathbf{y}$ as the measured observable parameters, and $\mathbf{V}_{\mathbf{y}}$ as the covariance matrix of measured data. The goal of the kinematic fit is to minimize

$$
\chi^{2}=(\mathbf{y}-\boldsymbol{\eta})^{T} \mathbf{V}_{\mathbf{y}}^{-1}(\mathbf{y}-\boldsymbol{\eta})
$$

subject to the constraints imposed by our hypothesis (usually implemented as Lagrange multipliers). The result of the fit is a set of improved measurements $\boldsymbol{\eta}$ and their covariance matrix $\mathbf{V}_{\boldsymbol{\eta}}$. Additional details of the kinematic fitter for the GlueX experiment can be found in Paul Mattione's magnum opus [40]. The improved four-vectors and vertex positions that result from the kinematic fit are used in the remainder of the analysis.

Kinematic fitting is sensitive to the accuracy of the parameters and error matrices returned by the particle reconstruction that serve as input to the routines. To test the validity of this fit given 
the early stage of reconstruction validation, the distribution of the "confidence level" (CL) of the kinematic fit can be inspected. Assuming the weighted residuals of the measured kinematics to our hypothesis are distributed as the standard normal $(\mu=0, \sigma=1)$, we may define the probability that a random $\chi^{2}$ will exceed the measured value, i.e.

$$
P\left(\chi^{2}, N D F\right)=1-C D F\left(\chi^{2}, N D F\right)
$$

where $C D F$ is the cumulative distribution function of a $\chi^{2}$ statistic with $N D F$ degrees of freedom. This probability is referred to as the confidence level of the fit. If our assumptions about the distributions of the weighted residuals hold, this value should be uniformly distributed from 0 to 1 . Thus by inspecting the shape of this distribution, we can make some rough statements about the quality of the fit itself.

Plots of the kinematic fit confidence level for all combinations passing the loose selection cuts in section 4.3 are shown in figure 4.2. In this figure, we have plotted the invariant mass of our candidate $\omega$ against the $\log$ of the kinematic fit confidence level. Our signal combinations are clearly clustered at high CL. In the $\pi^{+} \pi^{-} \pi^{0}$ decay plot, there is indication of exclusive $\eta(549), \omega(782)$ and $\phi(1020)$ production clearly separated from the background at high CL. Since $\omega$ is the only particle with a sizable branch to $\pi^{0} \gamma$, it is the only resonance that shows up at high CL in figure 4.2b. Combinations with a confidence level less than 0.01 are cut in the analysis that follows.

After applying additional cuts described in subsequent sections, we arrive at the distribution of the kinematic fit confidence level found in figure 4.3. Typically there is pileup at low confidence level due to deviations from Gaussian experimental errors. At high confidence level, these distributions should be flat if the errors reported for the particles are accurate. In the hadronic decay this seems to be the case. In the radiative decay there is a downward slope that may indicate these errors are being underestimated in the kinematic fit. An additional useful quantity to inspect are the pulls of the fits, defined for each measurement as

$$
z_{i} \equiv \frac{y_{i}-\eta_{i}}{\sigma\left(y_{i}-\eta_{i}\right)}
$$

with

$$
\begin{aligned}
\sigma^{2}\left(y_{i}-\eta_{i}\right) & =\left(\mathbf{V}_{\mathbf{y}}\right)_{i i}-2 \operatorname{cov}(\mathbf{y}, \boldsymbol{\eta})_{i i}+\left(\mathbf{V}_{\boldsymbol{\eta}}\right)_{i i} \\
& =\left(\mathbf{V}_{\mathbf{y}}\right)_{i i}-\left(\mathbf{V}_{\boldsymbol{\eta}}\right)_{i i}
\end{aligned}
$$

As an example, distributions of the kinematic-fit pulls for the individual components of momenta and position of the recoil proton in the reaction $\gamma p \rightarrow p \pi^{+} \pi^{-} \pi^{0}$ are shown in Figure 4.4. These are fit with a Gaussian, and the corresponding mean $(\mu)$ and standard deviation $(\sigma)$ are extracted. Tables 4.2 and 4.3 contain the pull means and widths for each of the reconstructed particles used as input to the kinematic fits. These results still show some small biases $(\mu \neq 0)$ and improper modeling of the error $(\sigma \neq 1)$, particularly in the neutral reconstruction. Detailed studies are still ongoing to validate our understanding of the experimental errors. These discrepancies from ideal behavior can cause a small systematic uncertainty associated with the kinematic fit results presented in this thesis. The systematic uncertainty associated with the cut value on the kinematic-fit confidence level is investigated in section 5.4.

\subsection{Additional Exclusivity Cuts}

\subsubsection{Measured Missing 4-Momenta}

To ensure that strong outliers are not passing our kinematic-fit confidence level cut due to improper errors, we inspect the measured kinematics that have not been improved by kinematic fitting. The 


\begin{tabular}{c|cccccc} 
Proton Pulls & $p_{x}$ & $p_{y}$ & $p_{z}$ & $x$ & $y$ & $z$ \\
\hline$\mu$ & -0.131 & 0.030 & -0.047 & 0.004 & -0.067 & -0.055 \\
$\sigma$ & 1.167 & 1.160 & 1.234 & 1.039 & 1.036 & 1.057 \\
$\pi^{+}$Pulls & $p_{x}$ & $p_{y}$ & $p_{z}$ & $x$ & $y$ & $z$ \\
\hline$\mu$ & -0.015 & -0.072 & 0.019 & 0.033 & 0.087 & 0.053 \\
$\sigma$ & 1.107 & 1.084 & 1.297 & 1.039 & 1.031 & 0.996 \\
$\pi^{-}$Pulls & $p_{x}$ & $p_{y}$ & $p_{z}$ & $x$ & $y$ & $z$ \\
\hline$\mu$ & -0.149 & 0.016 & 0.120 & 0.060 & 0.017 & 0.062 \\
$\sigma$ & 1.139 & 1.125 & 1.328 & 1.048 & 1.045 & 1.034 \\
$\gamma$ Pulls & & Energy & & $x$ & $y$ & $z$ \\
\hline$\mu$ & & -0.005 & & -0.263 & -0.033 & -0.010 \\
$\sigma$ & & 1.480 & & 1.334 & 1.329 & 1.443
\end{tabular}

Table 4.2: Mean and width of Gaussian fits to the pull distributions for the kinematic fit to $\gamma p \rightarrow$ $\pi^{+} \pi^{-} \pi^{0}$ requiring four-momentum conservation and a common vertex location. The $\pi^{0}$ mass is constrained in the fit.

\begin{tabular}{c|ccc} 
Proton Pulls & $p_{x}$ & $p_{y}$ & $p_{z}$ \\
\hline$\mu$ & -0.121 & 0.033 & 0.053 \\
$\sigma$ & 1.287 & 1.256 & 1.302 \\
Prompt $\gamma$ Pulls & $p_{x}$ & $p_{y}$ & $p_{z}$ \\
\hline$\mu$ & -0.090 & 0.015 & -0.078 \\
$\sigma$ & 1.330 & 1.304 & 1.436 \\
$\gamma$ from $\pi^{0}$ Pulls & $p_{x}$ & $p_{y}$ & $p_{z}$ \\
\hline$\mu$ & -0.098 & 0.015 & -0.107 \\
$\sigma$ & 1.360 & 1.361 & 1.440
\end{tabular}

Table 4.3: Mean and width of Gaussian fits to the pull distributions for the kinematic fit to $\gamma p \rightarrow \pi^{0} \gamma$ requiring four-momentum conservation. The $\pi^{0}$ mass is constrained in the fit. 


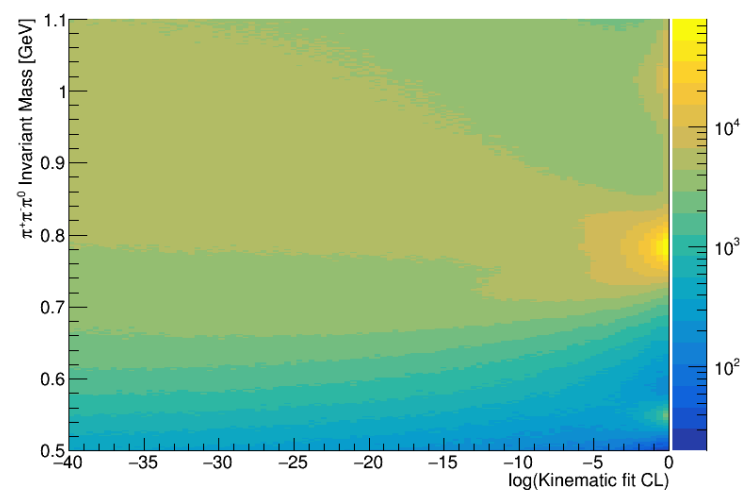

(a) $\gamma p \rightarrow p \pi^{+} \pi^{-} \pi^{0}$

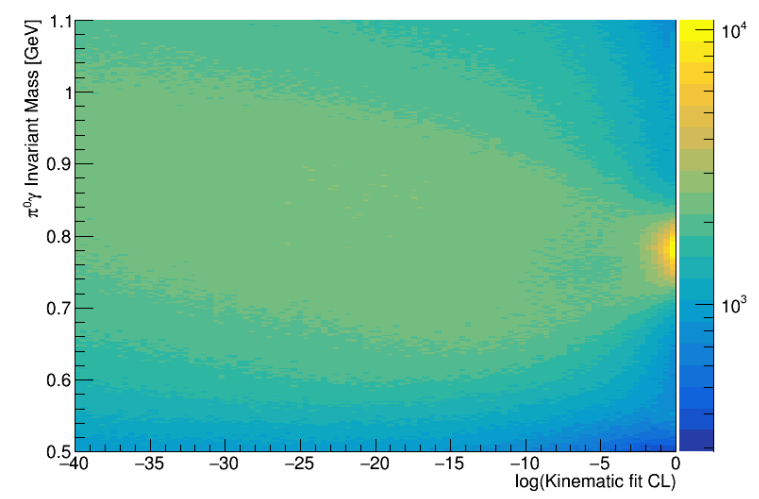

(b) $\gamma p \rightarrow p \pi^{0} \gamma$

Figure 4.2: $\pi^{+} \pi^{-} \pi^{0}$ and $\pi^{0} \gamma$ invariant mass as a function of kinematic fit confidence level.

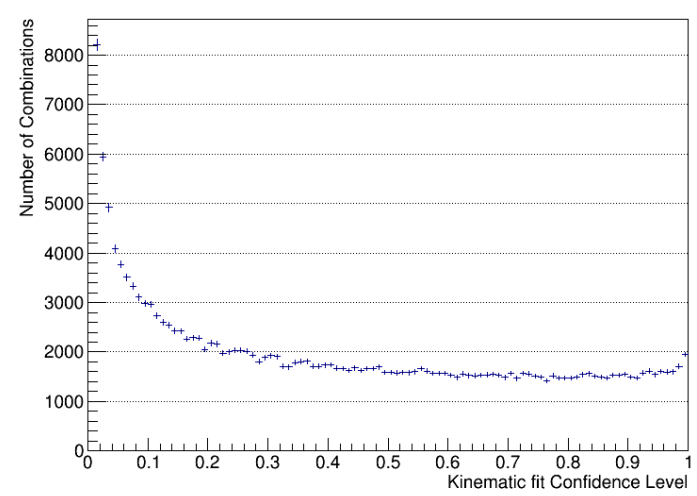

(a) $\gamma p \rightarrow p \pi^{+} \pi^{-} \pi^{0}$

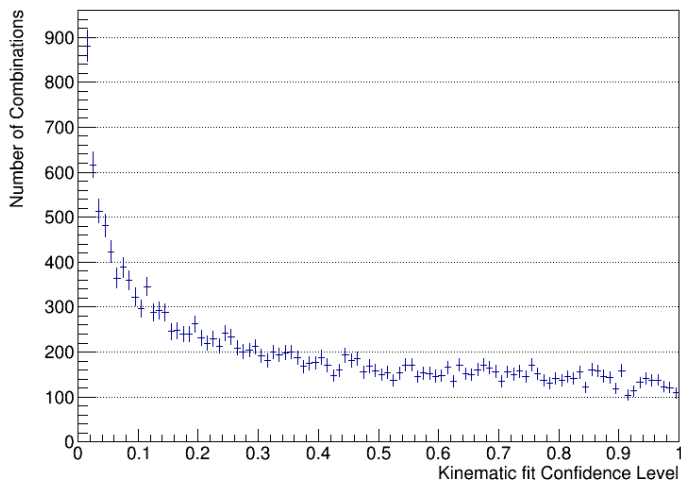

(b) $\gamma p \rightarrow p \pi^{0} \gamma$

Figure 4.3: Accidental-subtracted kinematic fit confidence level for all particle combinations in the golden run period passing all selection cuts presented in this chapter.

missing four-momentum of a given combination is

$$
p_{\text {Missing }}=\sum_{i=1}^{N_{i}} p_{\text {Measured }}^{i}-\sum_{f=1}^{N_{f}} p_{\text {Measured }}^{f}
$$

where the index $i$ runs over the particles in the initial state, and $f$ those in the final state. The missing mass squared of the combination is given by

$$
M M^{2}=E_{M i s s i n g}^{2}-\mathbf{p}_{M i s s i n g}^{2}
$$

The accidentals-subtracted missing-mass-squared is plotted for the hadronic and radiative decay of the omega in Figure 4.5. This plot includes all other selection cuts placed on the data. In both decays, the value is centered tightly around zero and there is very little background. In reality, our previous cut on the kinematic fit CL ensured this would be the case. A very loose cut is placed at $\pm 0.05 \mathrm{GeV}^{2}$ to reject any spurious combinations that may be outliers. We can also inspect the 

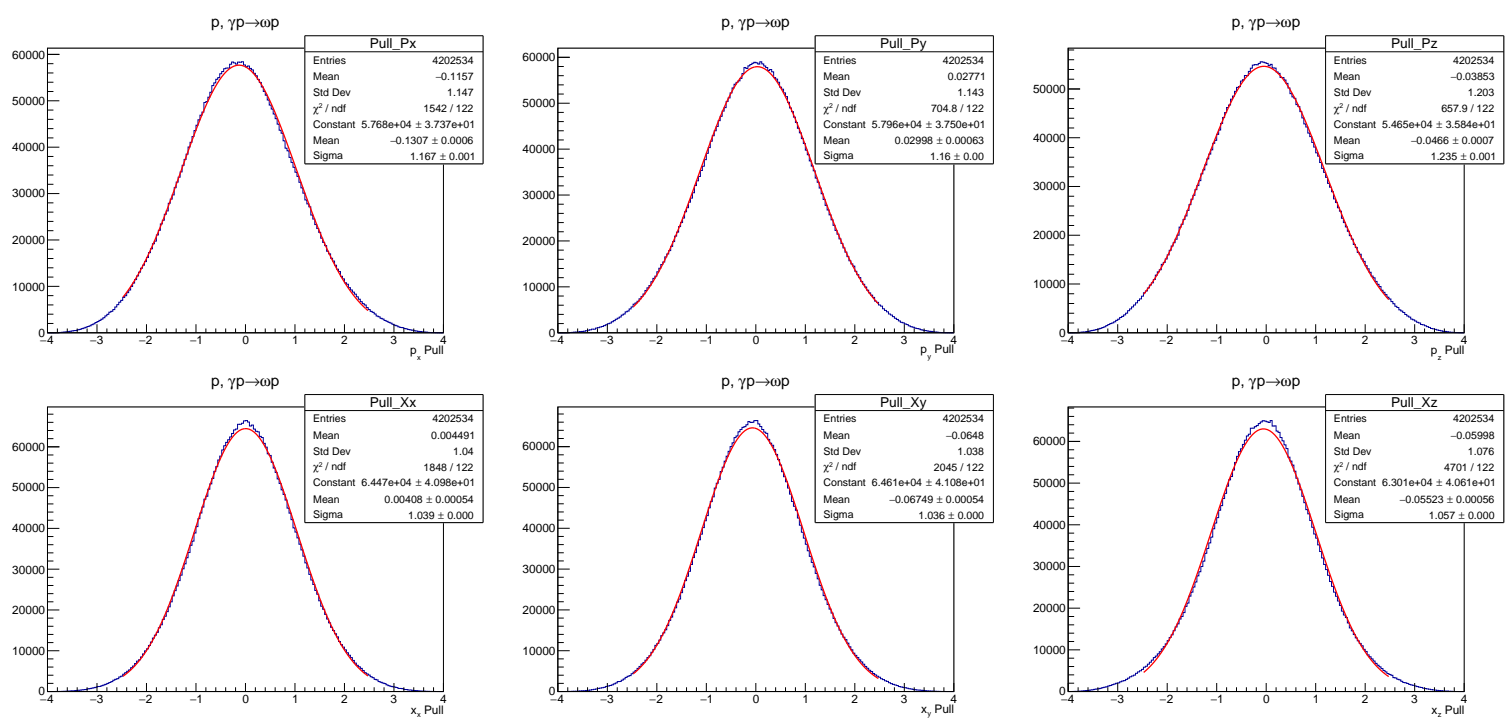

Figure 4.4: Pull distributions for the reconstructed proton in a kinematic fit to $\gamma p \rightarrow p \pi^{+} \pi^{-} \pi^{0}$ requiring four-momentum conservation and a common vertex location. Fits with a confidence level lower that 0.025 are excluded from these plots. The $\pi^{0}$ mass is constrained in the fit.

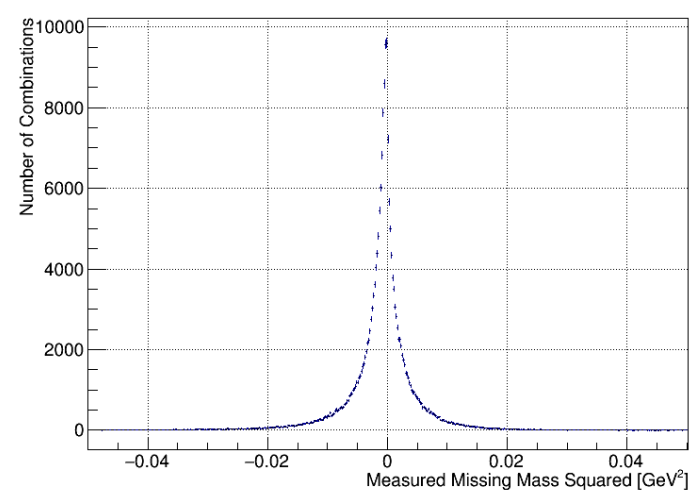

(a) $\gamma p \rightarrow p \pi^{+} \pi^{-} \pi^{0}$

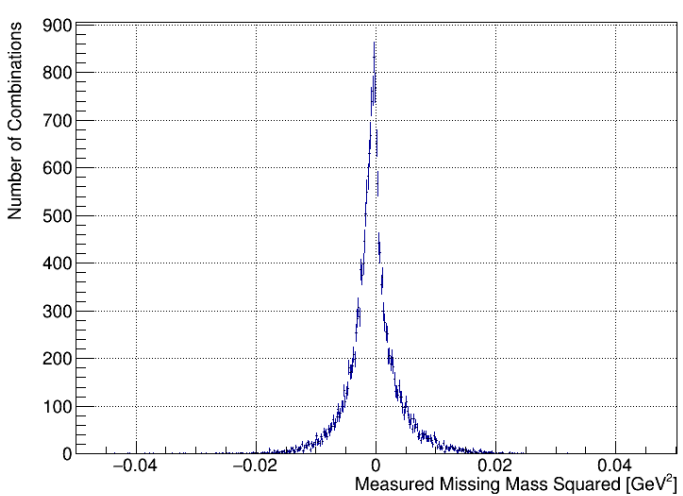

(b) $\gamma p \rightarrow p \pi^{0} \gamma$

Figure 4.5: Accidentals-subtracted measured missing-mass-squared. All other analysis cuts are applied.

individual components of the missing four-momentum. The accidentals-subtracted missing energy is shown in Figure 4.6. A cut is placed at $\pm 1.0 \mathrm{GeV}$ and is indicated on the plot. The accidentalssubtracted missing transverse momentum is shown in figure 4.7 . A cut is placed at $0.25 \mathrm{GeV}$ in the analysis that follows. 


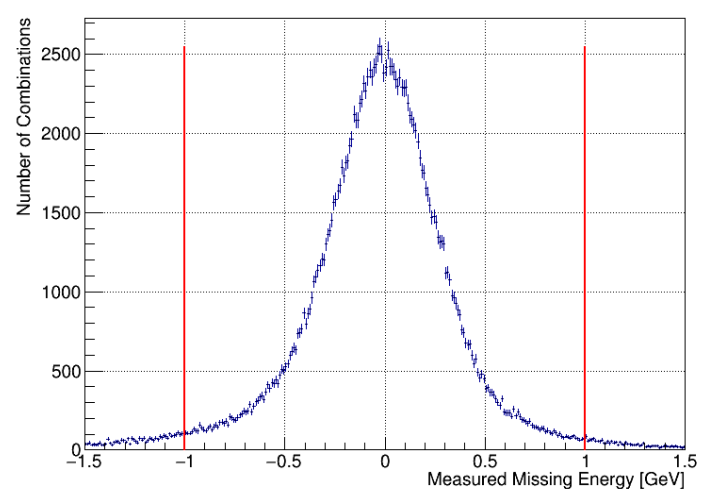

(a) $\gamma p \rightarrow p \pi^{+} \pi^{-} \pi^{0}$

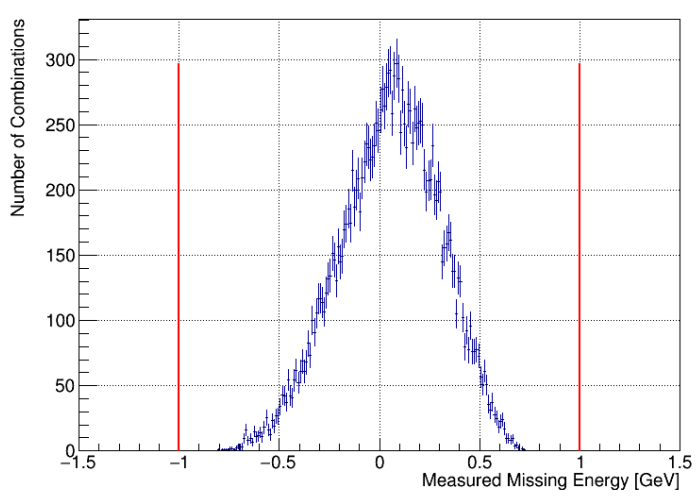

(b) $\gamma p \rightarrow p \pi^{0} \gamma$

Figure 4.6: Accidentals-subtracted measured missing energy. All other analysis cuts are applied.

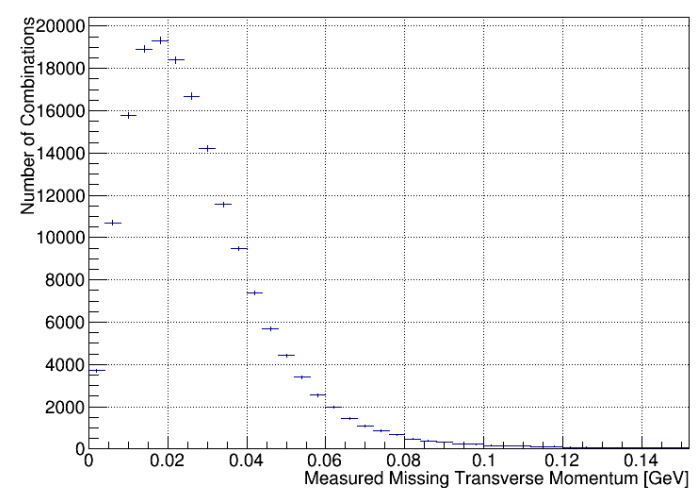

(a) $\gamma p \rightarrow p \pi^{+} \pi^{-} \pi^{0}$

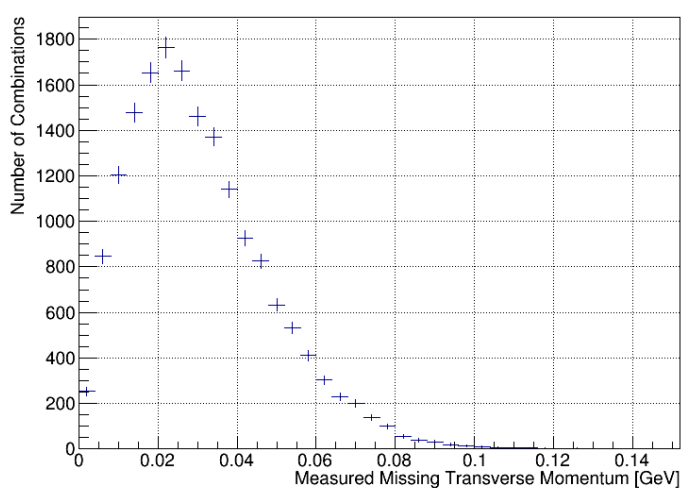

(b) $\gamma p \rightarrow p \pi^{0} \gamma$

Figure 4.7: Accidentals-subtracted measured missing transverse momentum. All other analysis cuts are applied.

\subsubsection{Measured $\pi^{0}$ Mass}

We may also inspect the measured $\pi^{0}$ invariant mass. The kinematic fit constrains the mass to the correct PDG value. The measured invariant mass distributions are found in figure 4.8. The tails in this distribution are cut as indicated in the figure. The measurement of the SDMEs in chapter 5 are not strongly influenced by the value of this cut. 


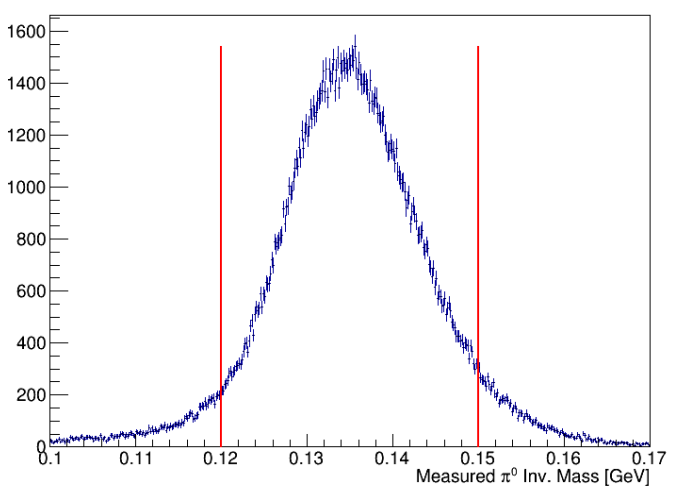

(a)

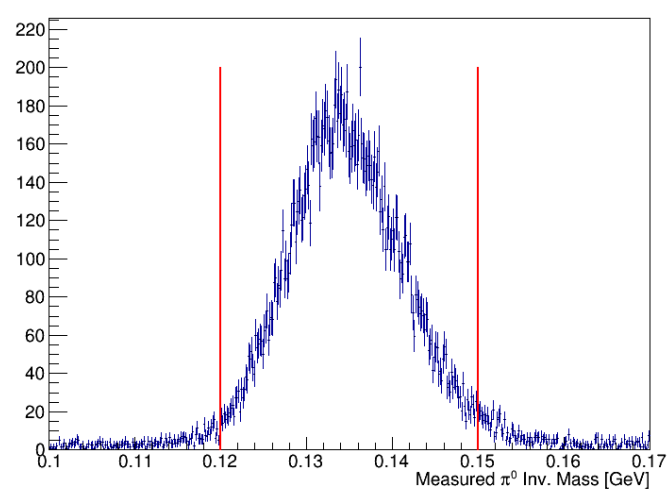

(b)

Figure 4.8: Accidentals-subtracted $\pi^{0}$ invariant mass for the (a) hadronic and (b) radiative decay.

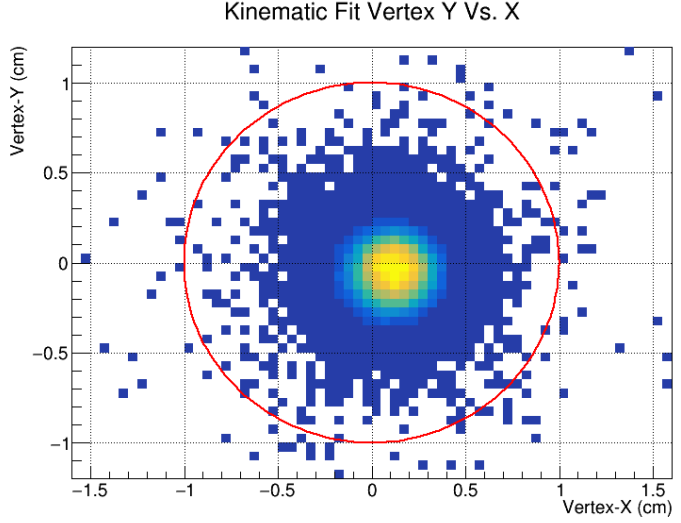

(a)

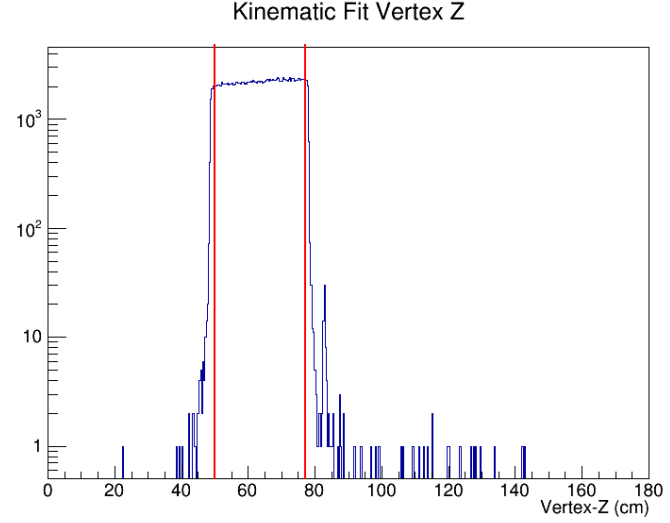

(b)

Figure 4.9: Kinematic fit vertex position (a) versus $x y$ (b) versus $z$. Selected regions are indicated by the red lines.

\subsubsection{Vertex Location}

Since we are interested in particle combinations originating in the hydrogen target, we place a cut on the vertex position returned by the kinematic fit. Plots of the kinematic fit vertex position are found in figure 4.9 and the corresponding cut regions are indicated. Since we require the recoil proton be detected in this exclusive measurement, there are very few events outside of the target in the $z$ projection.

\subsubsection{Measured Photon Kinematics}

In the fit for the spin-density-matrix elements presented in Chapter 5 it is required that we make a correction for the experimental acceptance. One critical component of this correction is correctly modeling the photon reconstruction. At the time of the writing of this thesis, inefficient FCAL blocks have not been removed from the reconstruction at the hit level. As an ad-hoc correction, we may mask certain regions of the detector based on the shower position. A plot of the FCAL shower 


\section{FCAL Shower Position}

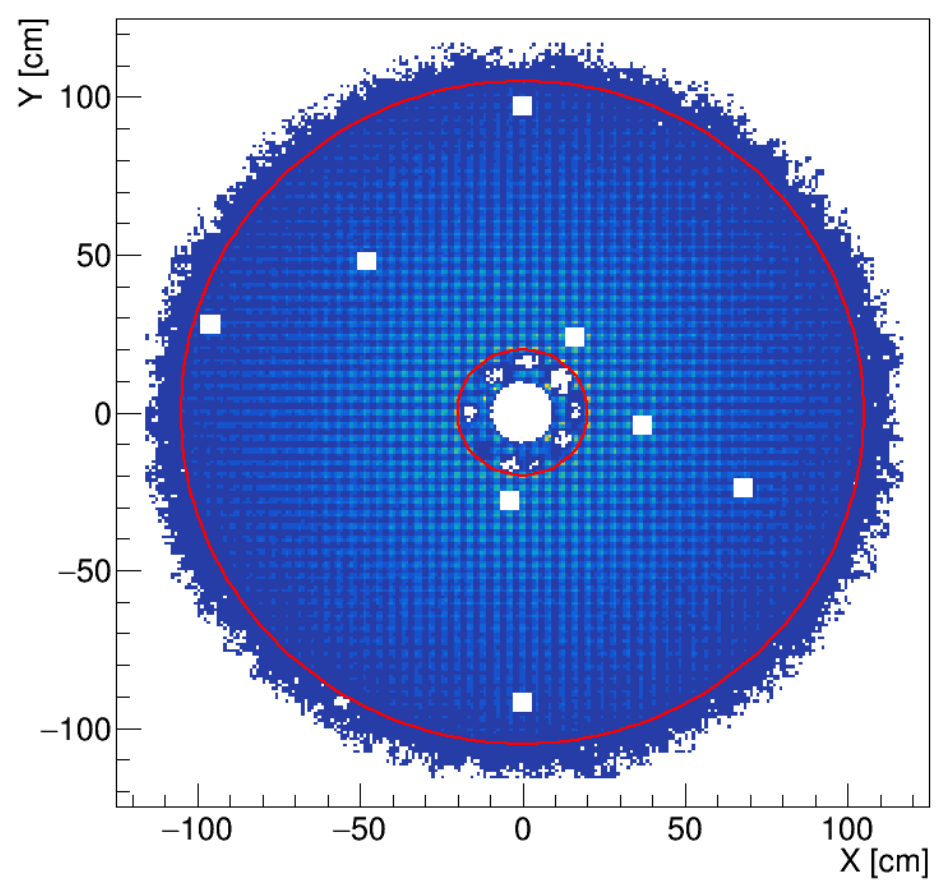

Figure 4.10: FCAL shower positions in the hadronic decay. The region between the two circles are selected for the analysis. Individual problematic channels in the accepted region have been excluded from the analysis.

position for photons passing our selection cuts is found in figure 4.10. There are clear issues with the reconstruction near the beamline. This region is excluded by a circular cut around the deficient region. The outer region of the detector is also cut as there is some overlap with the BCAL acceptance in this region that is poorly modeled. Regions surrounding individual problematic channels in the accepted region have also been masked in the analysis.

The reconstructed photon position in the BCAL is plotted in figure 4.11. The very forward region is cut as low-angle-of-incidence showers require more study before inclusion in the analysis. This forward cut could be relaxed as validation of the Monte Carlo proceeds. The cut near the middle of the detector is driven by low accepted Monte Carlo statistics below this z position. This can be addressed through a larger-scale production of Monte Carlo data for the acceptance correction. For this analysis, this is unnecessary since it is found that the value of this cut does not greatly impact our final measurement. In the radiative decay, the BCAL is completely excluded from the analysis. Including the BCAL region causes large uncertainties in the measured SDMEs. The cause of this effect is not yet understood. The systematic uncertainty ascribed to this cut is studied in section 5.4 .

\subsubsection{Particle Identification Based on $\mathrm{dE} / \mathrm{dx}$}

A useful tool for selecting between different mass-hypotheses for charged tracks is measurements of the energy loss along the track from interactions with the detector medium. The CDC is well suited for this measurement as discussed earlier in section 2.4.1. Typically a cut on this value is used to separate positively charged pions from protons at momenta less than $1 \mathrm{GeV}$. However, after 


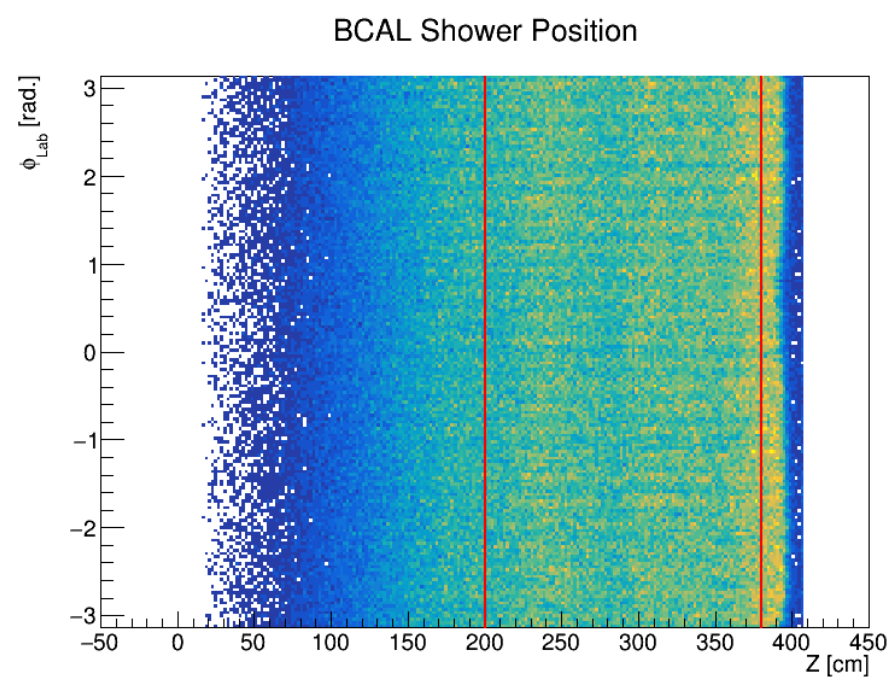

Figure 4.11: BCAL shower positions in the hadronic decay. The region between the two lines is selected for the analysis.

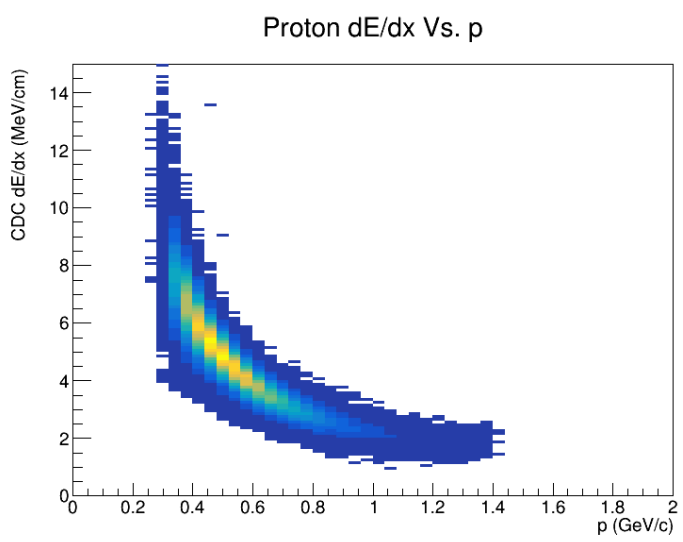

(a)

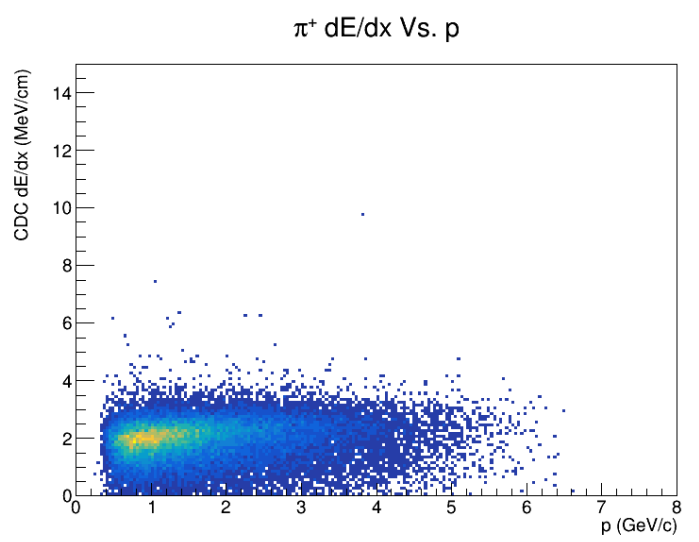

(b)

Figure 4.12: $\mathrm{dE} / \mathrm{dx}$ versus measured particle momentum for (a) protons and (b) positively charged pions as identified by kinematic fit and missing-mass cuts. There is clear separation of the proton from the $\pi^{+}$.

applying the exclusivity cuts described in previous chapters, we find additional cuts based on this quantity to be unnecessary for the analysis. The distributions of the energy deposition per unit length for positive particles are shown in figure 4.12 as a function of the particle momentum. There is little sign of misidentification of the two particle types.

\subsubsection{Particle Identification Based on Time-of-Flight}

An additional method for discriminating between particle types is using the time-of-flight measurements from the target to the different timing detector elements. For charged particles, energy loss may be modeled along the track and factored into the expected hit time. At the first analysis stage, there is a loose cut on each of the timing measurements of \pm 4 ns. After the exclusivity cuts described 


\begin{tabular}{|c|c|}
\hline Quantity & Nominal Cut Value \\
\hline Kinematic Fit Confidence Level & 0.01 \\
Measured Missing Mass Squared & {$[-0.05,0.05] \mathrm{GeV}^{2}$} \\
Maximum Measured Missing $p_{t}$ & $0.25 \mathrm{GeV}$ \\
Measured Missing Energy & {$[-1.0,1.0] \mathrm{GeV}$} \\
Photon Beam Energy & {$[8.4,9.0] \mathrm{GeV}$} \\
Measured $\pi^{0}$ Invariant Mass & {$[0.12,0.15] \mathrm{GeV}$} \\
Kinematic-Fit $\omega$ Invariant Mass & {$[0.76,0.81] \mathrm{GeV}$} \\
Kinematic-Fit-Vertex Z & {$[50.0,77.0] \mathrm{cm}$} \\
Maximum Kinematic-Fit Vertex R & $1.0 \mathrm{~cm}$ \\
BCAL Neutral Shower Z & {$[200.0,380.0] \mathrm{cm}$} \\
FCAL Neutral Shower R & {$[20.0,100.0] \mathrm{cm}$} \\
Kinematic-Fit Proton $\theta_{l a b}$ & {$\left[52.0^{\circ}, 78.0^{\circ}\right]$} \\
Minimum Kinematic-Fit Pion $\theta_{l a b}$ & $1.0^{\circ}$ \\
\hline
\end{tabular}

Table 4.4: Summary of analysis cuts performed to extract our exclusive $\omega$ signal.

in this chapter, the difference of the tagged photon RF-time and the target-time of the particle in its primary timing detectors is plotted in figure 4.13. The spurious events that are present outside of the main bands are not necessarily consistent with misidentification of the particle. These would appear as separate diverging bands instead of random noise. For this analysis, no additional cut is placed on the timing. The choice to not place a cut is also motivated by the early stage of ongoing work to match timing resolutions between Monte Carlo and data.

\subsubsection{Summary of Analysis Cuts}

A summary of the analysis cuts placed on our candidate particle combinations can be found in table 4.4. After applying these cuts the effective number of signal combinations surviving after accidental subtraction is 156,973 in the hadronic decay and 18,398 in the radiative decay. The PARA and PERP data samples have been combined and no cuts on Mandelstam $t$ are applied for this estimate.

\subsection{Combinations Passing Analysis Cuts}

\subsection{1 $\omega$ Invariant Mass}

With all of the cuts applied to the data, we can plot the invariant mass of our candidate $\omega$ measured in decays to $\pi^{+} \pi^{-} \pi^{0}$ and $\pi^{0} \gamma$. These distributions are shown in figures 4.14 and 4.15. Measured experimental resonance lineshapes are typically well approximated by the convolution of a nonrelativistic Breit-Wigner (Lorentzian) function that describes the resonance, and a Gaussian that describes detector resolution. this function is known as a Voigtian. The invariant-mass distributions are fit with the sum of a Voightian profile and a linear background. These fits indicate a sample of high purity in both decay modes. The quoted purity is obviously dependent on the lineshape and background model. In the hadronic decay, the fit does not seem to describe the peak or the right tail of the peak very well. This will impact forthcoming measurements of the cross sections for this reaction, but should not affect the measurements presented in this thesis. For the radiative decay, the lineshape describes the data quite well, albeit with a higher Lorentzian width than the PDG value $(14.8 \mathrm{MeV}$ vs. $8.5 \mathrm{MeV})$. Aside from the analysis presented here, this high-purity sample will be useful for additional detailed studies of the detector performance ongoing within the collaboration. 


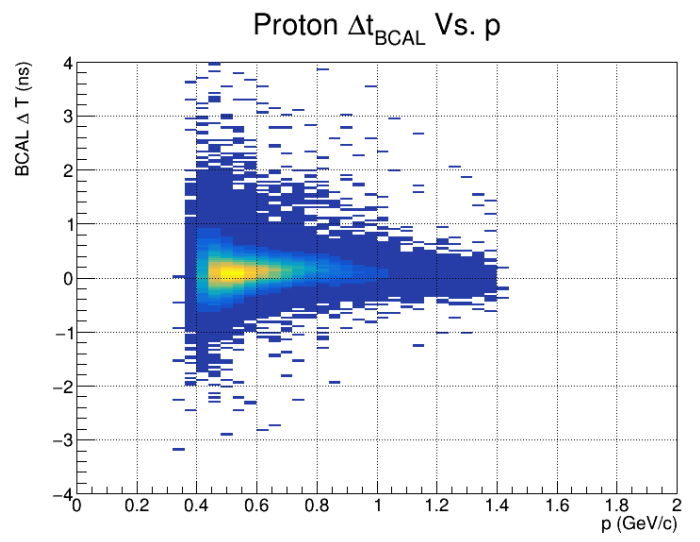

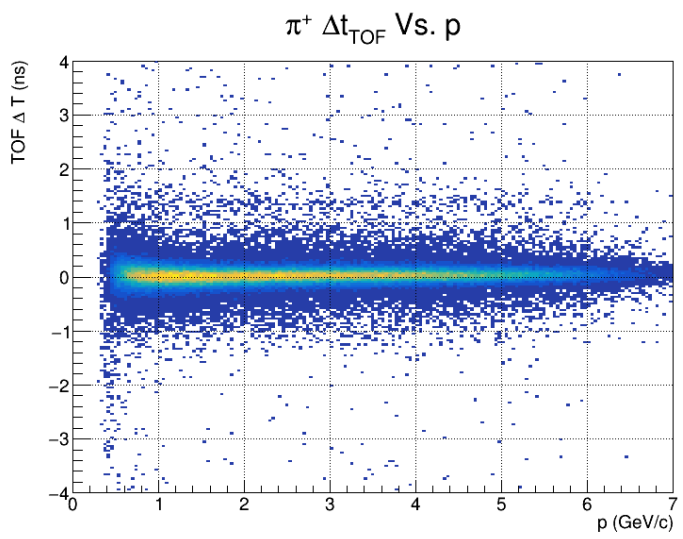

(b)

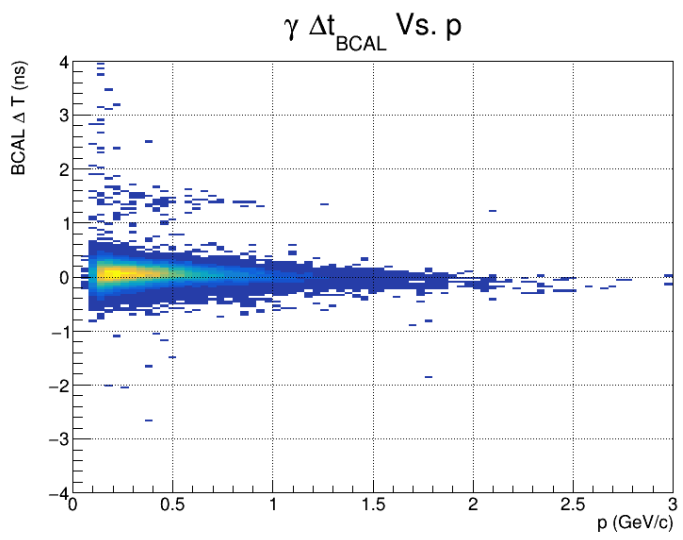

(d)

(a)

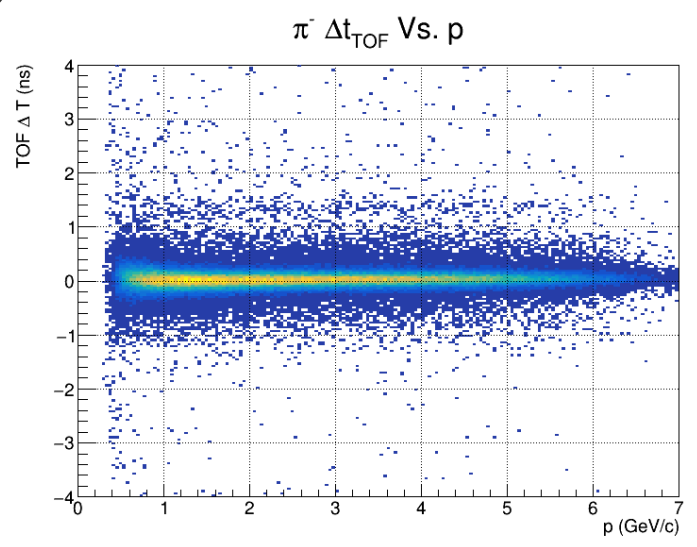

(c)

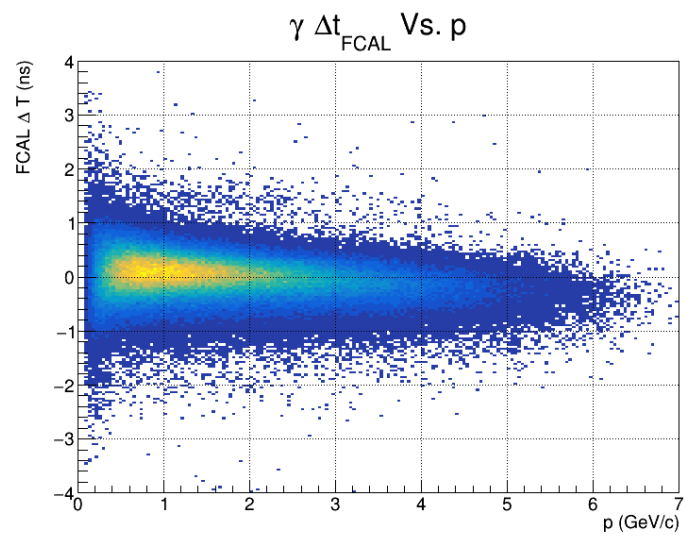

(e)

Figure 4.13: $\triangle$ TOF versus measured particle momentum for (a) proton candidates hitting the BCAL, (b) $\pi^{+}$candidates hitting the TOF, (c) $\pi^{-}$candidates hitting the TOF, (d) photon candidates hitting the BCAL, and (e) photon candidates hitting the FCAL. There is no indication of misidentified background. No additional cut is applied on these values. 


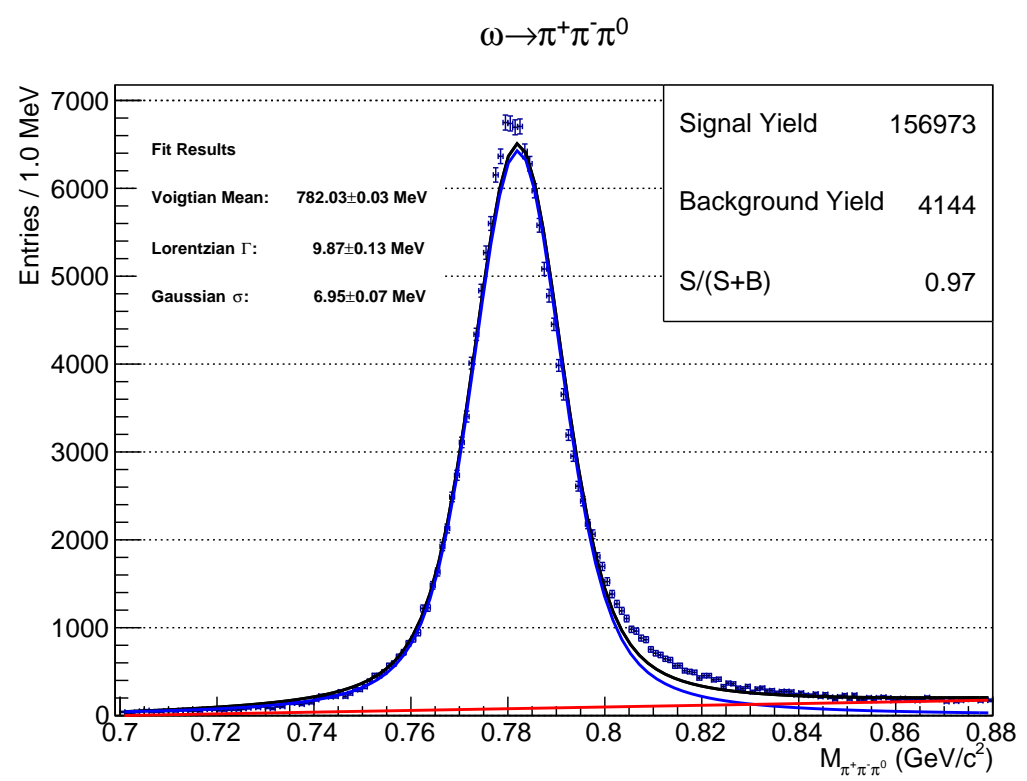

Figure 4.14: Accidental-subtracted $\pi^{+} \pi^{-} \pi^{0}$ invariant mass for all combinations passing selection cuts. The peak is fit with a Voightian plus a linear background. The signal and background integrals are performed over the mass range of 0.76 to $0.81 \mathrm{GeV}$ selected for analysis.

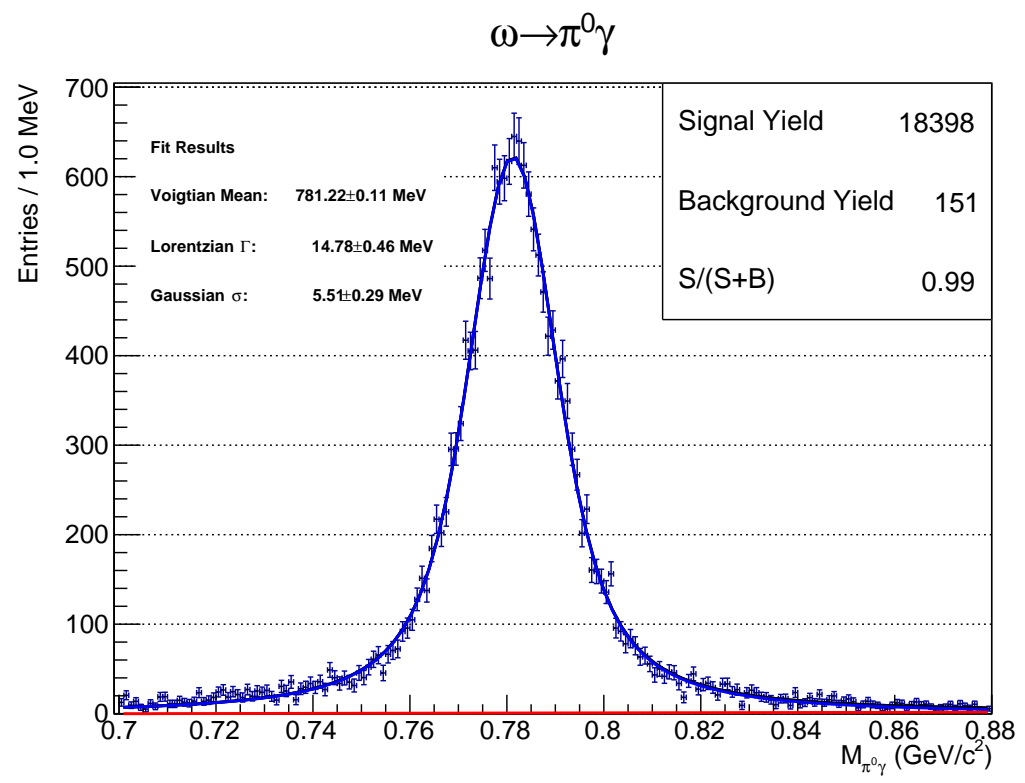

Figure 4.15: Accidental-subtracted $\pi^{0} \gamma$ invariant mass for all combinations passing selection cuts. The peak is fit with a Voightian plus a linear background. The signal and background integrals are performed over the mass range of 0.76 to $0.81 \mathrm{GeV}$ selected for analysis.

\subsection{2 $|t|$ Distribution}

For the 2-body to 2-body reaction $1+2 \rightarrow 3+4$, The Mandelstam variable $t$ is defined as

$$
t=\left|p_{1}-p_{3}\right|^{2}=\left|p_{2}-p_{4}\right|^{2} \text {. }
$$




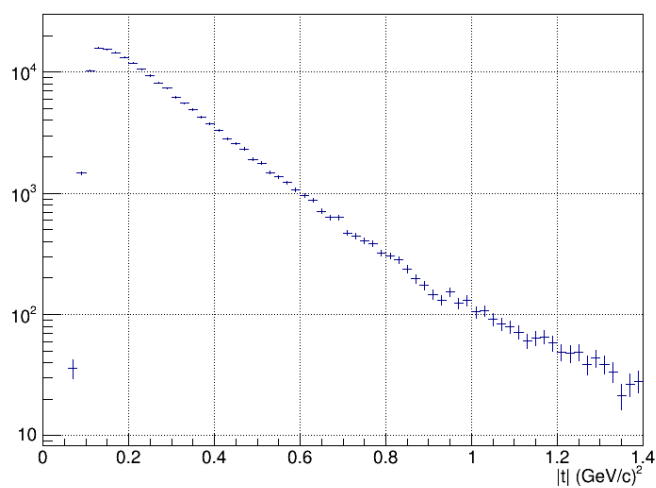

(a)

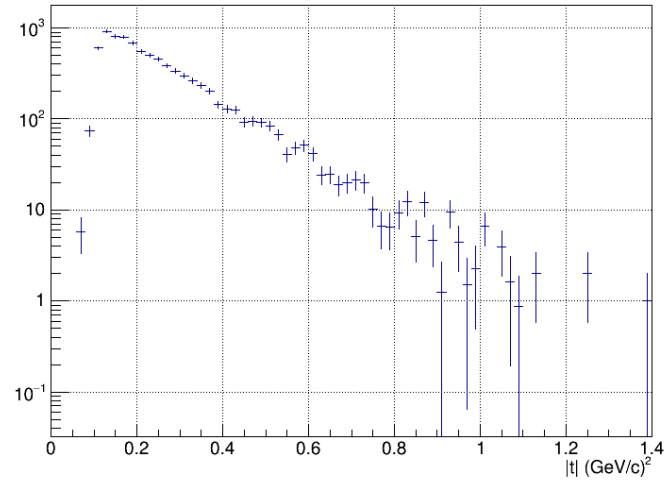

(b)

Figure 4.16: Mandelstam $|t|$ distribution for all combinations passing analysis cuts for the (a) hadronic and (b) radiative decay. The falloff at low $|t|$ is due to poor experimental acceptance. These results are not acceptance-corrected.

We use the kinematic-fit proton four-momentum and the four-momentum of our target proton (at rest in the lab frame) to calculate $t$ for each combination. The accidentals-subtracted $|t|$-distribution of the selected combinations is shown in 4.16. This distribution has the exponential falloff that is characteristic of diffractive $t$-channel production at low $|t|$. For the fits that follow in Chapter 5 , we have chosen to bin the data in four bins of $|t|$ for the hadronic decay ranging from 0.10 to $0.80 \mathrm{GeV}^{2}$. Due to lower statistics and large systematic uncertainties, the radiative decay is fit in a single bin of $|t|$ from 0.10 to $0.60 \mathrm{GeV}^{2}$. 


\section{Chapter 5}

\section{Measurement of Spin Density Matrix Elements}

In this Chapter, we perform a fit of the angular distributions presented in Section 1.4.3 to our experimental data selected in Chapter 4. We also discuss the systematic uncertainties associated with the measurement.

\subsection{Likelihood Fit}

In order to extract the SDMEs from the experimental data selected in Chapter 4, we perform an unbinned maximum likelihood fit using the AmpTools framework [41]. The AmpTools framework is designed to be an experiment-independent implementation of fitting routines for partial wave analyses. In this analysis, we are not fitting to the amplitudes, but instead directly fit the intensity given by equation 1.9 for each of the decay modes. While perhaps not the designed application, AmpTools still provides a useful set of tools for performing the acceptance correction for the fit, and displaying the results.

AmpTools is designed to fit experimental data in terms of physics amplitudes. The intensity as a function of the kinematics is given as

$$
I(\Omega)=\sum_{\alpha}\left|\sum_{\beta} V_{\alpha \beta} A_{\alpha \beta}(\Omega)\right|^{2} .
$$

The physics amplitudes $A_{\alpha \beta}$ are provided by the user for the desired reaction. The parameters to be fit are the complex production amplitudes $V_{\alpha \beta}$. The intensity is calculated as a coherent sum over $\beta$ and an incoherent sum over $\alpha$. The parameters required to calculate the physics amplitudes are labeled as $\Omega$.

In our specific case, there is only an incoherent sum over the intensity distribution modeled by $W(\cos \theta, \phi)$. The AmpTools framework provides the flexibility to fit parameters in the physics amplitudes as well as the $V_{\alpha \beta}$ coefficients. Our fit is therefore simply to the intensity

$$
I(\boldsymbol{\rho}, \Phi, \cos \theta, \phi)=|V|^{2} W(\boldsymbol{\rho}, \Phi, \phi, \cos \theta)
$$

where $\boldsymbol{\rho}$ are the measurable SDMEs, $\Phi$ is the angle of the production plane relative to the photon polarization direction, and $\cos \theta$ and $\phi$ are the decay angles in the helicity frame as described in Section 1.4.3. The parameters to be fit are the SDMEs, $\boldsymbol{\rho}$, and the overall normalization, $V$. In order to relate the modeled intensity to what is actually measured in the experiment, we must 
additionally include the experimental acceptance $\eta(\Omega)$. The number of predicted events including this acceptance function is given by

$$
N_{\text {predicted }} \equiv \mu=\int I(\Omega) \eta(\Omega) d \Omega .
$$

The fit is performed by maximizing the likelihood function

$$
\mathcal{L}=\frac{e^{-\mu} \mu^{N}}{N !} \prod_{i=1}^{N} \frac{I\left(\Omega_{i}\right) \eta\left(\Omega_{i}\right)}{\mu}
$$

where $\mu$ is the number of predicted events and $N$ is the number observed. One can imagine that in the process of minimization, derivatives of equation 5.4 with respect to the fit parameters could be quite unruly. Thankfully, it is an equivalent optimization problem to maximize the log likelihood. After taking the log likelihood, the product becomes a sum over the elements and the problem becomes numerically feasible [42]. Ignoring constant terms, we may write

$$
\ln \mathcal{L}=\sum_{i=1}^{N} \ln \left(\sum_{\alpha, \beta}^{N_{\mathrm{amps}}} V_{\alpha} V_{\beta}^{*} A_{\alpha}(\Omega) A_{\beta}(\Omega)^{*}\right)-\sum_{\alpha, \beta}^{N_{\mathrm{amps}}} V_{\alpha} V_{\beta}^{*} \int \eta(\Omega) A_{\alpha}(\Omega) A_{\beta}(\Omega)^{*} d \Omega .
$$

The normalization integral responsible for the acceptance correction can be calculated using Monte Carlo generated uniformly over the volume of phase space.

$$
\int \eta(\Omega) A_{\alpha}(\Omega) A_{\beta}(\Omega)^{*} d \Omega=\mathcal{V} \frac{1}{N_{\text {gen }}} \sum_{i=1}^{N_{\text {gen }}} \eta(\Omega) A_{\alpha}(\Omega) A_{\beta}(\Omega)^{*}
$$

where $\mathcal{V}$ is the volume of phase space and can be ignored in the optimization since it is a constant. $N_{\text {gen }}$ is the number of generated Monte Carlo events. The acceptance function $\eta(\Omega)$ is either 0 or 1 depending on whether the event was rejected or accepted. Thus we may write equation 5.5 as

$$
\int \eta(\Omega) A_{\alpha}(\Omega) A_{\beta}(\Omega)^{*} d \Omega \rightarrow \frac{1}{N_{\text {gen }}} \sum_{i=1}^{N_{\text {Accepted }}} A_{\alpha}(\Omega) A_{\beta}(\Omega)^{*}
$$

for the purpose of optimization [43].

There are four data samples that go into the AmpTools fit.

1. A Monte Carlo sample generated flat over the measured angles.

2. This same Monte Carlo sample processed through the detector simulation with the same analysis cuts as the data. These first two samples provide the input to the acceptance correction.

3. The data sample to be fit.

4. Additional "background" events. In this analysis, the only background considered are particle combinations arising from accidental photon tags.

The success of the minimization is sensitive to the initial parameters used in the fit. The starting values used for each of the parameters can be found in Table 5.1. These were determined by fitting the data with some parameters fixed. The results of these fits were then used as the starting points for fits with fewer fixed values until a reasonable stable set of initial parameters was found for the full fit. In order to investigate the effect of these starting values on the results after minimization, a study was performed by randomly selecting the initial parameters. There are few local minima 


\begin{tabular}{|c|c|c|}
\hline Parameter & Hadronic Decay & Radiative Decay \\
\hline \hline$\rho_{00}^{0}$ & 0.07 & 0.07 \\
\hline$\rho_{10}^{0}$ & 0.07 & 0.07 \\
\hline$\rho_{1-1}^{0}$ & -0.07 & -0.07 \\
\hline$\rho_{11}^{1}$ & -0.10 & -0.10 \\
\hline$\rho_{00}^{1}$ & 0.00 & 0.00 (fixed) \\
\hline$\rho_{10}^{1}$ & -0.10 & -0.10 \\
\hline$\rho_{1-1}^{1}$ & 0.20 & 0.35 \\
\hline$\rho_{10}^{2}$ & 0.00 & 0.10 \\
\hline$\rho_{1-1}^{2}$ & -0.20 & -0.35 \\
\hline
\end{tabular}

Table 5.1: Initial values used in AmpTools fit.

\begin{tabular}{|c|c|c|c|}
\hline Decay & MC Events Thrown & MC Events Accepted & Percent Accepted \\
\hline$\omega \rightarrow \pi^{+} \pi^{-} \pi^{0}$ & $7,880,000$ & 245,543 & $3.1 \%$ \\
\hline$\omega \rightarrow \pi^{0} \gamma$ & $9,880,000$ & 98,680 & $1.0 \%$ \\
\hline
\end{tabular}

Table 5.2: Number of thrown and accepted Monte Carlo events used for acceptance corrections.

observed, and the results reported are observed to be from the true global minimum of the negative log-likelihood when starting from the set of parameters in Table 5.1.

The nominal polarization directions are $\phi_{\|}=0^{\circ}$ and $\phi_{\perp}=90^{\circ}$ relative to the lab coordinates. However, a small offset exists in the data. Fitting a histogram of the accidentals-subtracted $\phi$ distribution of the decay plane in the $\omega$ helicity frame with a function of the form $f(\phi)=p_{0}+$ $p_{1} \cos \left(2\left(\phi-\phi_{0}\right)\right)$ yields $\phi_{0}=3.59 \pm 0.51^{\circ}$ for PARA polarization and $\phi_{0}=2.39 \pm 0.46^{\circ}$ for $\mathrm{PERP}$ polarization. Alternatively, this angle can be allowed to float in the AmpTools fit. Using this second method yields $\phi_{0}=3.08 \pm 0.20^{\circ}$ for PARA polarization and $\phi_{0}=3.35 \pm 0.24^{\circ}$ for PERP polarization. The two methods yield consistent results. The latter values are used in the fits that follow and are fixed in the minimization.

\subsection{Monte Carlo Sample for Acceptance Correction}

The generator used for our acceptance correction to the measured data was implemented using AmpTools. The intensity is the product of a Breit-Wigner describing the resonance, an exponential t-dependence describing the diffractive nature of the reaction, and the available phase space to the particular kinematics. The t-slope is taken to be $3.5 \mathrm{GeV}^{-2}$ to enhance the fraction of simulated events entering the geometrical experimental acceptance. This is smaller than previous measured values [15], but does not affect our fit results for the SDMEs. These thrown events are then processed with a Hall-D specific Geant3 based simulation tool called HDGeant. The data are then smeared according to instrumental resolutions in a program called mcsmear. These smeared data are then subjected to the same full set of analysis cuts as the real data. The number of thrown events, and the number passing all selection cuts are shown in Table 5.2.

\subsection{Fit Results}

The fit results for the hadronic decay in bins of $|t|$ are shown in Figure 5.1. The fit results for the radiative decay in a single bin of $|t|$ are shown in figure 5.2. In order to investigate the quality of the fit, we may plot the accidentals-subtracted data against our accepted Monte Carlo that has been re-weighted by the result of the fit. A comparison in the lowest t-bin in the hadronic PARA data set 
is shown in Figure 5.3. The results match quite well in each of the angular variables fit for the decay distribution. Due to relative sample sizes and incomplete understanding of the systematic effects, the radiative sample is more challenging to fit. A comparison in the lowest t-bin in the radiative PARA data set is shown in Figure 5.4. In the radiative sample, there is a strong acceptance effect in $\cos \theta$ at forward and backward angles, and in $\phi$ near zero and $\pi$ attributed to the excluded calorimeter regions. These appear to be modeled fairly well, but additional investigation of the quality of the acceptance correction may be needed in the future.

In the fits for the radiative decay, the $\rho^{1}$ elements show strong correlations leading to large statistical errors in the fit parameters. The problem is mitigated by fixing one of the parameters. In order to investigate the effect this has on the final parameters, the value is varied by $\pm 2 \sigma$ of the measurement in the hadronic channel and included in the systematic uncertainties determined in Section 5.4. $\rho_{00}^{1}$ was chosen since the hadronic measurements for this parameter are uniformly consistent with zero across the measured $t$ range. 

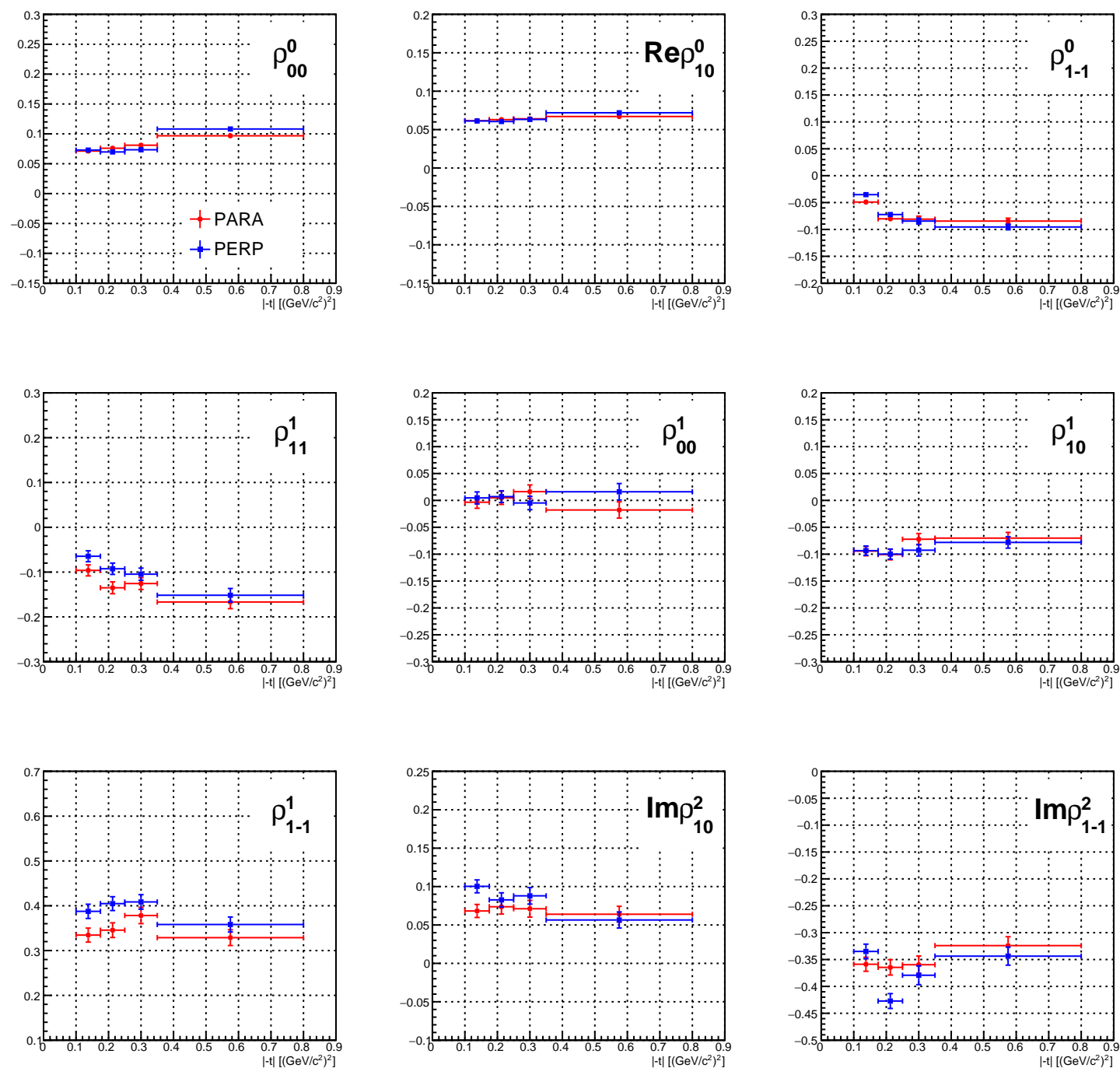

Figure 5.1: Fit results for the measurable SDMEs in the hadronic decay channel for the two separate polarization orientations. Error bars indicate only statistical errors returned by AmpTools. 

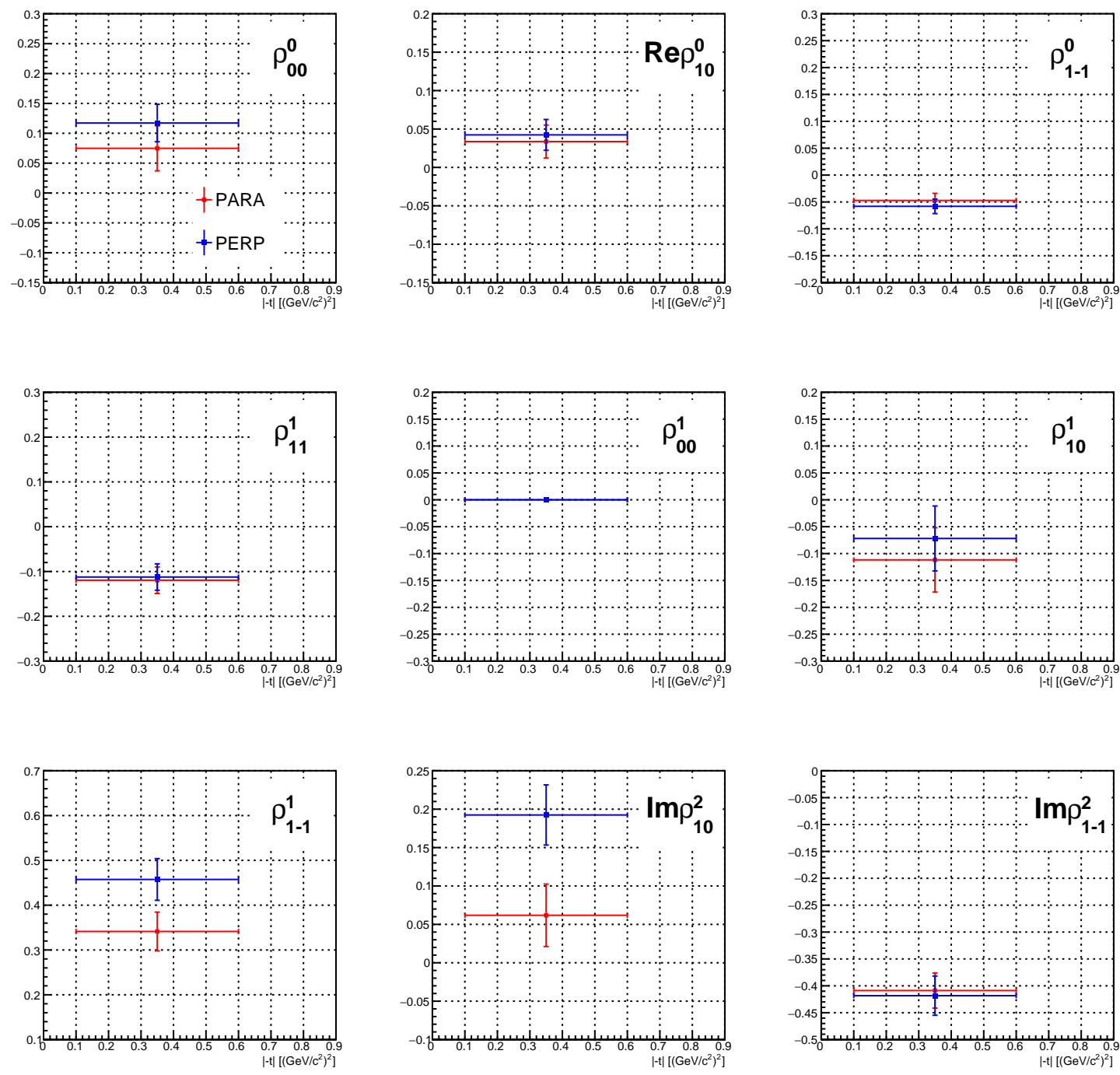

Figure 5.2: Fit results for the measurable SDMEs in the radiative decay channel for the two separate polarization orientations. SDME $\rho_{00}^{1}$ is fixed in the fit. Error bars indicate only statistical errors returned by AmpTools. Parameter $\rho_{00}^{1}$ is fixed in the minimization. 

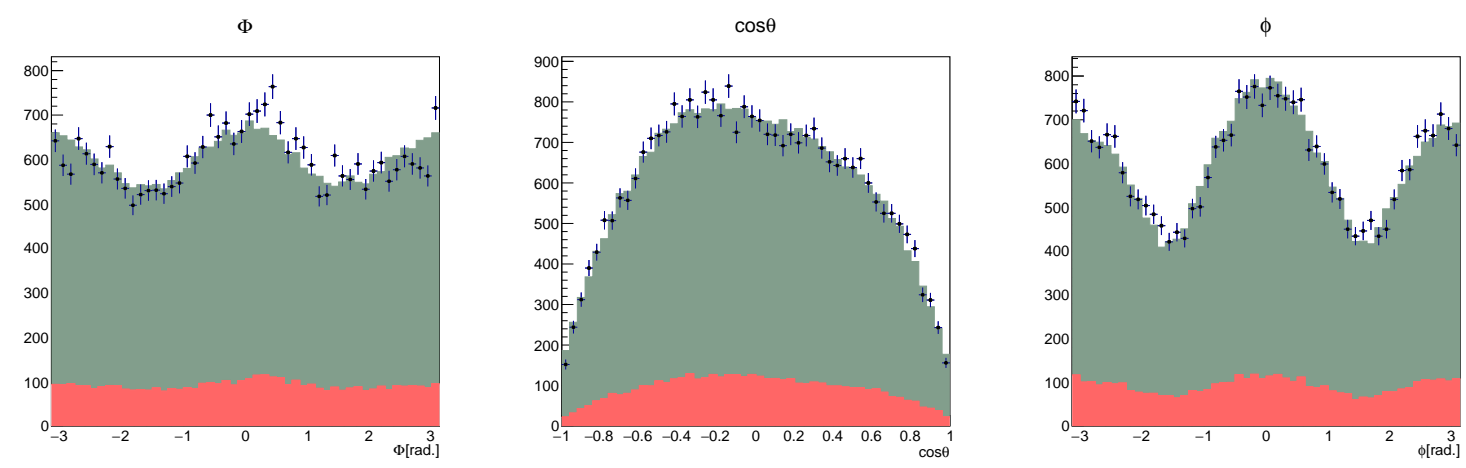

Figure 5.3: Comparison of data with the result of the fit for $|t| \in[0.100,0.350), \omega \rightarrow \pi^{+} \pi^{-} \pi^{0}$ decay, PARA polarization. The points are the experimental data, the red filled histogram is the contribution from the tagger-accidental background, and the green filled histogram is the re-weighted accepted Monte Carlo. The angle $\Phi$ is the angle of the decay plane relative to the photon polarization vector, and $\cos \theta$ and $\phi$ are the decay angles in the helicity frame as described in Section 1.4.3.

$\Phi$

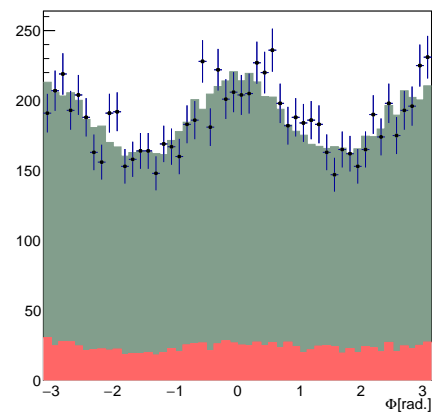

$\cos \theta$

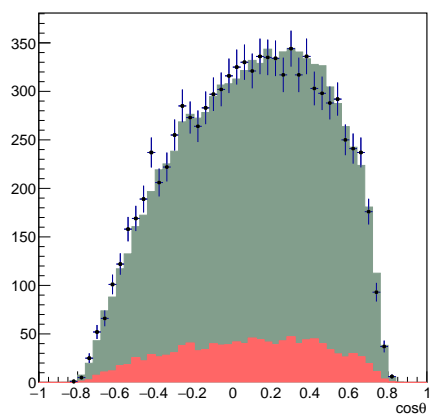

$\phi$

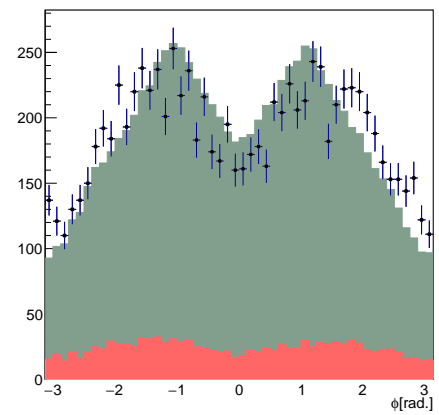

Figure 5.4: Comparison of data with the result of the fit for $|t| \in[0.100,0.600), \omega \rightarrow \pi^{0} \gamma$ decay, PARA polarization. The points are the experimental data, the red filled histogram is the contribution from the tagger-accidental background, and the green filled histogram is the re-weighted accepted Monte Carlo. The angle $\Phi$ is the angle of the decay plane relative to the photon polarization vector, and $\cos \theta$ and $\phi$ are the decay angles in the helicity frame as described in Section 1.4.3. 


\subsection{Systematics Studies}

According to the fits in Section 5.3 there is a discrepancy in the SDMEs extracted for the two perpendicular polarization states. These SDME measurements represent the first physics results to come from the GlueX experiment that require the experimental acceptance to be correctly modeled. At the time of writing this thesis, studies are still ongoing to appropriately match Monte Carlo detector resolutions and efficiencies with data. The SDMEs presented here use an acceptance correction based on the current state of the Monte Carlo that has had minimal tuning to match the data. It is a testament to the quality of the simulation that the results achieved are even nearly consistent with minimal tuning. In this section, we will attempt to quantify the effect of our selection cuts on the measured SDMEs. We will then discuss other remaining systematics that may affect the measurement.

The exact placement of the selection cuts presented in Table 4.4 is somewhat arbitrary. In order to estimate the systematic uncertainty caused by this selection, each of the cut parameters is varied around the selected value. These samples with the adjusted cut are then fit for the SDMEs. The standard deviation of the measurements in this ensemble are taken to be the systematic uncertainty associated with this selection. The t-bin size has been widened for these studies to minimize the statistical error that may impact this measurement.

Consider the following example. One of the analysis cuts is on the minimum distance between a reconstructed photon and the beamline in the FCAL. The nominal cut value is $\gamma_{R}>20.0 \mathrm{~cm}$. We can vary this cut in steps of $1 \mathrm{~cm}$ from $15 \mathrm{~cm}$ to $25 \mathrm{~cm}$. The data and Monte Carlo samples are then reprocessed with the new cut value before a new fit is performed. The variation of the measured SDMEs with respect to this cut parameter is shown for the two decay modes in Figures 5.5 and 5.6. In the hadronic decay, there is little effect on the measured values of the SDME by changing this cut, but in the radiative decay there is a very strong dependence. The exact cause of this dependence is unknown. Using the prescription above, we assign the following values to the systematic uncertainty arising from this cut in the two decay modes. The values are listed in Table 5.3. This process is then repeated for all of the cuts placed on the data. The total systematic error is determined by adding all of the individual components in quadrature. This assumes the individual contributions are uncorrelated. It is also assumed that the systematic uncertainties do not vary with $t$. In Table 5.4 we present the total systematic uncertainties assigned to each of the SDMEs based on varying our input parameters to the analysis. A full table of the individual components of the systematic uncertainties can be found in Appendix B.

\begin{tabular}{|c|c|c|c|c|c|c|c|c|c|}
\hline Dataset & $\sigma \rho_{00}^{0}$ & $\sigma \rho_{10}^{0}$ & $\sigma \rho_{1-1}^{0}$ & $\sigma \rho_{11}^{1}$ & $\sigma \rho_{00}^{1}$ & $\sigma \rho_{10}^{1}$ & $\sigma \rho_{1-1}^{1}$ & $\sigma \rho_{10}^{2}$ & $\sigma \rho_{1-1}^{2}$ \\
\hline Hadronic Decay PARA & 0.002 & 0.003 & 0.003 & 0.001 & 0.001 & 0.001 & 0.002 & 0.003 & 0.003 \\
Hadronic Decay PERP & 0.001 & 0.003 & 0.003 & 0.002 & 0.001 & 0.001 & 0.002 & 0.001 & 0.002 \\
Radiative Decay PARA & 0.022 & 0.033 & 0.023 & 0.013 & fixed & 0.016 & 0.016 & 0.013 & 0.016 \\
Radiative Decay PERP & 0.019 & 0.033 & 0.024 & 0.017 & fixed & 0.028 & 0.017 & 0.021 & 0.010 \\
\hline
\end{tabular}

Table 5.3: Systematic uncertainties assigned to the SDMEs for variations of minimum FCAL R cut.

\begin{tabular}{|c|c|c|c|c|c|c|c|c|c|}
\hline Dataset & $\sigma \rho_{00}^{0}$ & $\sigma \rho_{10}^{0}$ & $\sigma \rho_{1-1}^{0}$ & $\sigma \rho_{11}^{1}$ & $\sigma \rho_{00}^{1}$ & $\sigma \rho_{10}^{1}$ & $\sigma \rho_{1-1}^{1}$ & $\sigma \rho_{10}^{2}$ & $\sigma \rho_{1-1}^{2}$ \\
\hline Hadronic Decay PARA & 0.018 & 0.021 & 0.030 & 0.018 & 0.014 & 0.014 & 0.041 & 0.010 & 0.031 \\
Hadronic Decay PERP & 0.018 & 0.020 & 0.032 & 0.019 & 0.009 & 0.019 & 0.035 & 0.012 & 0.031 \\
Radiative Decay PARA & 0.090 & 0.073 & 0.077 & 0.044 & fixed & 0.055 & 0.057 & 0.046 & 0.049 \\
Radiative Decay PERP & 0.089 & 0.075 & 0.076 & 0.047 & fixed & 0.072 & 0.056 & 0.045 & 0.068 \\
\hline
\end{tabular}

Table 5.4: Systematic uncertainties assigned to the SDMEs based on parameter variation. 
PARA SDME Vs dMinFCALR

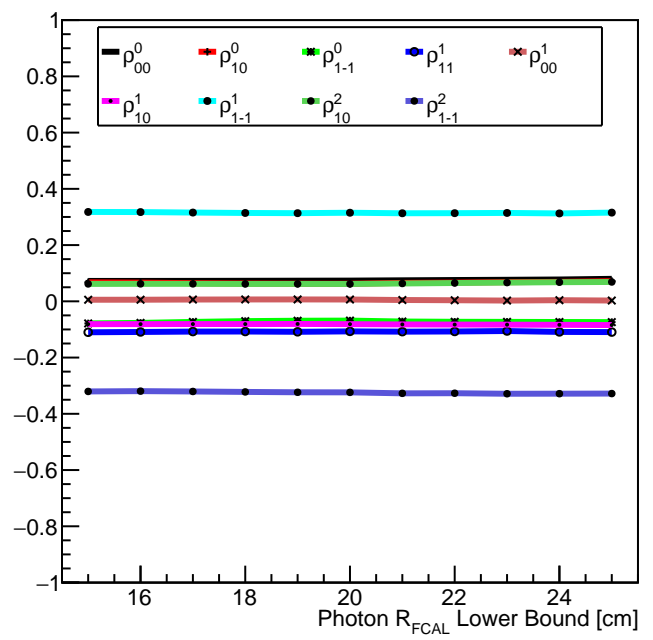

PERP SDME Vs dMinFCALR

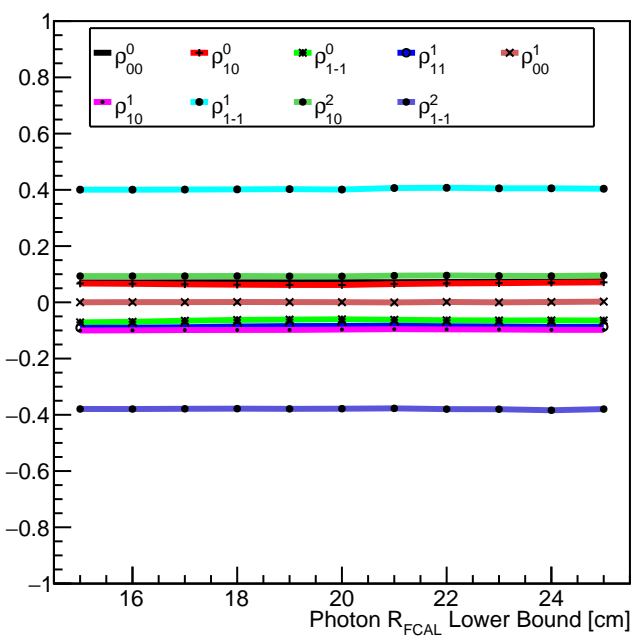

Figure 5.5: Measured SDMEs for variations of the minimum photon FCAL radius cut for the hadronic decay $\omega \rightarrow \pi^{+} \pi^{-} \pi^{0}$ in PARA and PERP polarization orientations.

PARA SDME Vs dMinFCALR

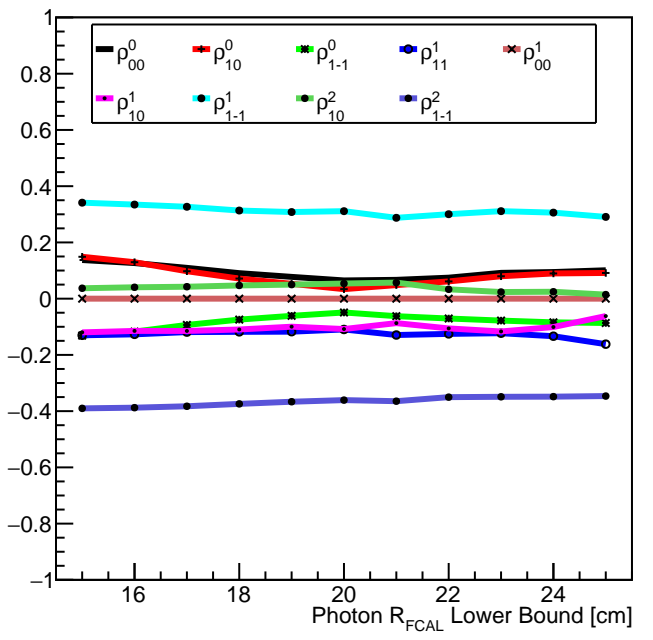

PERP SDME Vs dMinFCALR

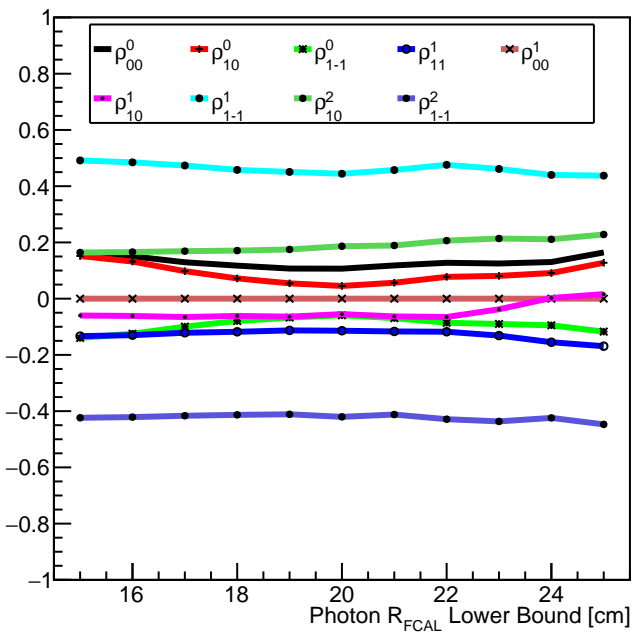

Figure 5.6: Measured SDMEs for variations of the minimum photon FCAL radius cut for the radiative decay $\omega \rightarrow \pi^{0} \gamma$ in PARA and PERP polarization orientations. 
The fit results for the SDMEs including the systematic errors assigned in Table 5.4 are found in Figures 5.7 and 5.8. After including the systematic effects our selection criteria have on our measurements, the PARA and PERP datasets mostly agree to within errors. We assign one additional systematic uncertainty in combining the results of the two datasets. Our final result is taken to be the average of the SDMEs in each t-bin. Half of the average discrepancy between PARA and PERP polarization fits in each bin is taken as the additional systematic and is added in quadrature to the average systematic uncertainties from Table 5.4. The systematic error assigned to each configuration for the PARA/PERP discrepancy is presented in Table 5.5. The final results including the total statistical and reported systematic uncertainty are collected in Table 5.7. Comparisons of these results and the OTL model presented in Section 1.5 for both decay modes are shown in Figures 5.9
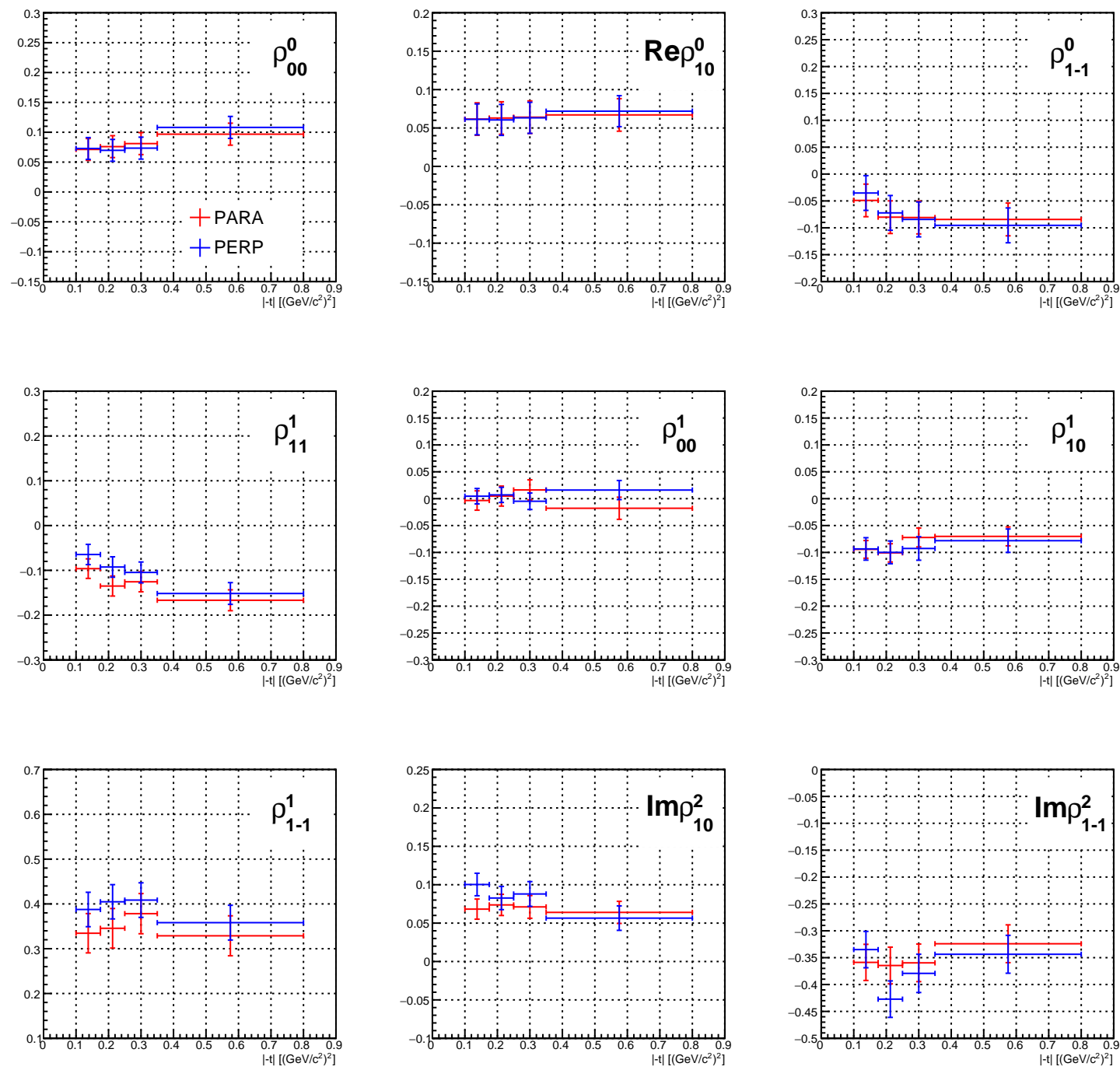

Figure 5.7: Fit results for the measurable SDMEs in the $\omega \rightarrow \pi^{+} \pi^{-} \pi^{0}$ decay channel for the two separate polarization orientations. Error bars indicate the quadrature addition of statistical errors returned by AmpTools and the systematic uncertainties assigned in Table 5.4. 


\begin{tabular}{|c|c|c|c|c|c|c|c|c|c|}
\hline Dataset & $\sigma \rho_{00}^{0}$ & $\sigma \rho_{10}^{0}$ & $\sigma \rho_{1-1}^{0}$ & $\sigma \rho_{11}^{1}$ & $\sigma \rho_{00}^{1}$ & $\sigma \rho_{10}^{1}$ & $\sigma \rho_{1-1}^{1}$ & $\sigma \rho_{10}^{2}$ & $\sigma \rho_{1-1}^{2}$ \\
\hline Hadronic Decay & 0.003 & 0.001 & 0.005 & 0.014 & 0.008 & 0.004 & 0.021 & 0.008 & 0.016 \\
Radiative Decay & 0.021 & 0.004 & 0.005 & 0.004 & fixed & 0.020 & 0.058 & 0.065 & 0.005 \\
\hline
\end{tabular}

Table 5.5: Systematic uncertainties assigned to the SDMEs to address the PARA/PERP discrepancy.

\begin{tabular}{|c|c|c|c|c|c|c|c|c|c|}
\hline Dataset & $\sigma \rho_{00}^{0}$ & $\sigma \rho_{10}^{0}$ & $\sigma \rho_{1-1}^{0}$ & $\sigma \rho_{11}^{1}$ & $\sigma \rho_{00}^{1}$ & $\sigma \rho_{10}^{1}$ & $\sigma \rho_{1-1}^{1}$ & $\sigma \rho_{10}^{2}$ & $\sigma \rho_{1-1}^{2}$ \\
\hline Hadronic Decay & 0.018 & 0.021 & 0.031 & 0.023 & 0.014 & 0.017 & 0.043 & 0.014 & 0.035 \\
Radiative Decay & 0.092 & 0.074 & 0.077 & 0.046 & 0.000 & 0.067 & 0.081 & 0.079 & 0.059 \\
\hline
\end{tabular}

Table 5.6: Total systematic uncertainties assigned to the SDMEs described in Section 5.4.

and 5.10 .

\subsection{Discussion of Results}

In this chapter we have presented preliminary measurements of the SDMEs in $\omega$ photoproduction at $E_{\gamma}=8.4-9.0 \mathrm{GeV}$ with the GlueX detector. Results are presented in two decay modes and are in agreement according to our current understanding of the experimental uncertainties. These results are generally consistent with a simple model based on Pomeron and pseudoscalar t-channel exchange mechanisms. However, there is some significant deviation from the model in some of the elements. It will be important to investigate if this result holds up to continued scrutiny. Deviations from the OTL model in $\rho_{1-1}^{1}$ and $\operatorname{Im} \rho_{1-1}^{2}$ could be indicative of higher relative Pomeron contribution to the production. The effect this has on the predicted SDMEs is shown in Figure 5.11. The Pomeron contribution must be enhanced by roughly $20 \%$ to match the behavior observed in the data in the two most significant non-zero elements, $\rho_{1-1}^{1}$ and $\operatorname{Im} \rho_{1-1}^{2}$. The results are also consistent with earlier measurements at $E_{\gamma}=9.3 \mathrm{GeV}$ at SLAC [15], though the earlier measurements suffer from limited statistics.

\begin{tabular}{|c|c|c|c|c|c|}
\hline Decay Mode & \multicolumn{4}{|c|}{$\omega \rightarrow \pi^{+} \pi^{-} \pi^{0}$} & $\omega \rightarrow \pi^{0} \gamma$ \\
\hline$|\mathrm{t}|$-bin $\left[\mathrm{GeV}^{2}\right]$ & $0.100-0.175$ & $0.175-0.250$ & $0.250-0.350$ & $0.350-0.800$ & $0.100-0.600$ \\
\hline$\rho_{00}^{0}$ & $0.072 \pm 0.019$ & $0.073 \pm 0.019$ & $0.077 \pm 0.019$ & $0.102 \pm 0.019$ & $0.096 \pm 0.104$ \\
$\rho_{10}^{0}$ & $0.061 \pm 0.021$ & $0.062 \pm 0.021$ & $0.064 \pm 0.021$ & $0.069 \pm 0.021$ & $0.038 \pm 0.080$ \\
$\rho_{1-1}^{0}$ & $-0.042 \pm 0.032$ & $-0.076 \pm 0.032$ & $-0.083 \pm 0.032$ & $-0.090 \pm 0.032$ & $-0.053 \pm 0.079$ \\
$\rho_{11}^{1}$ & $-0.080 \pm 0.029$ & $-0.114 \pm 0.030$ & $-0.115 \pm 0.030$ & $-0.159 \pm 0.031$ & $-0.116 \pm 0.062$ \\
$\rho_{00}^{1}$ & $0.001 \pm 0.021$ & $0.006 \pm 0.022$ & $0.006 \pm 0.023$ & $-0.001 \pm 0.026$ & fixed at 0.0 \\
$\rho_{10}^{1}$ & $-0.094 \pm 0.021$ & $-0.100 \pm 0.022$ & $-0.082 \pm 0.023$ & $-0.074 \pm 0.023$ & $-0.092 \pm 0.108$ \\
$\rho_{1-1}^{1}$ & $0.361 \pm 0.049$ & $0.375 \pm 0.049$ & $0.393 \pm 0.050$ & $0.343 \pm 0.050$ & $0.399 \pm 0.103$ \\
$\rho_{10}^{2}$ & $0.084 \pm 0.018$ & $0.078 \pm 0.019$ & $0.080 \pm 0.020$ & $0.060 \pm 0.020$ & $0.127 \pm 0.097$ \\
$\rho_{1-1}^{2}$ & $-0.347 \pm 0.040$ & $-0.396 \pm 0.040$ & $-0.369 \pm 0.042$ & $-0.334 \pm 0.042$ & $-0.414 \pm 0.076$ \\
\hline
\end{tabular}

Table 5.7: Combined results for PARA and PERP polarization datasets. The error is the combined statistical and systematic error. 

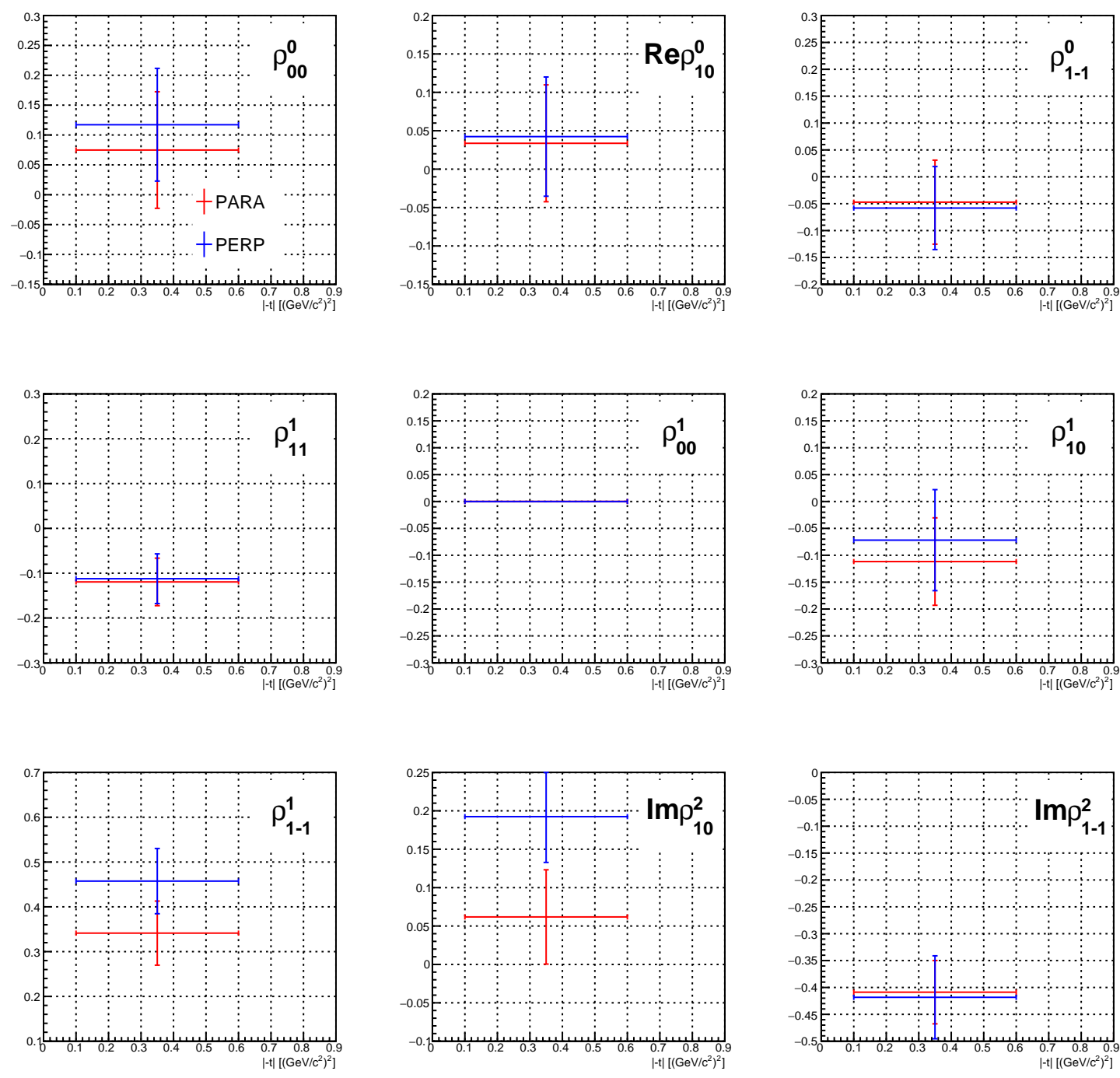

Figure 5.8: Fit results for the measurable SDMEs in the $\omega \rightarrow \pi^{0} \gamma$ decay channel for the two separate polarization orientations. Error bars indicate the quadrature addition of statistical errors returned by AmpTools and the systematic uncertainties assigned in Table 5.4. Parameter $\rho_{00}^{1}$ is fixed in the minimization. 

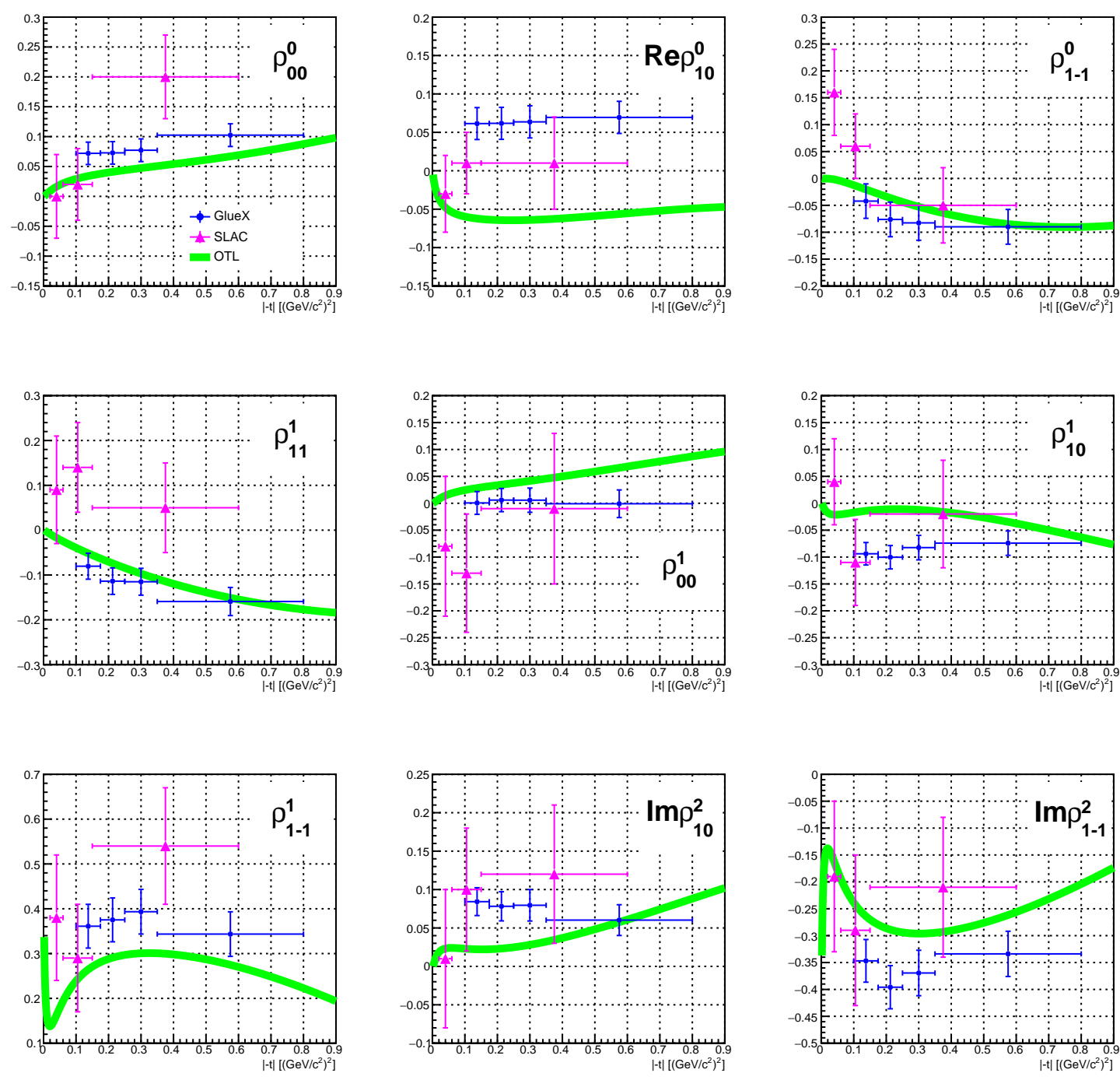

Figure 5.9: Fit results for the measurable SDMEs in the hadronic decay channel. Error bars indicate the quadrature addition of statistical errors returned by AmpTools and the systematic uncertainties assigned in Table 5.6. This result is compared with measurements made at SLAC [15] and predictions from the Oh, Titov, Lee model [11]. 

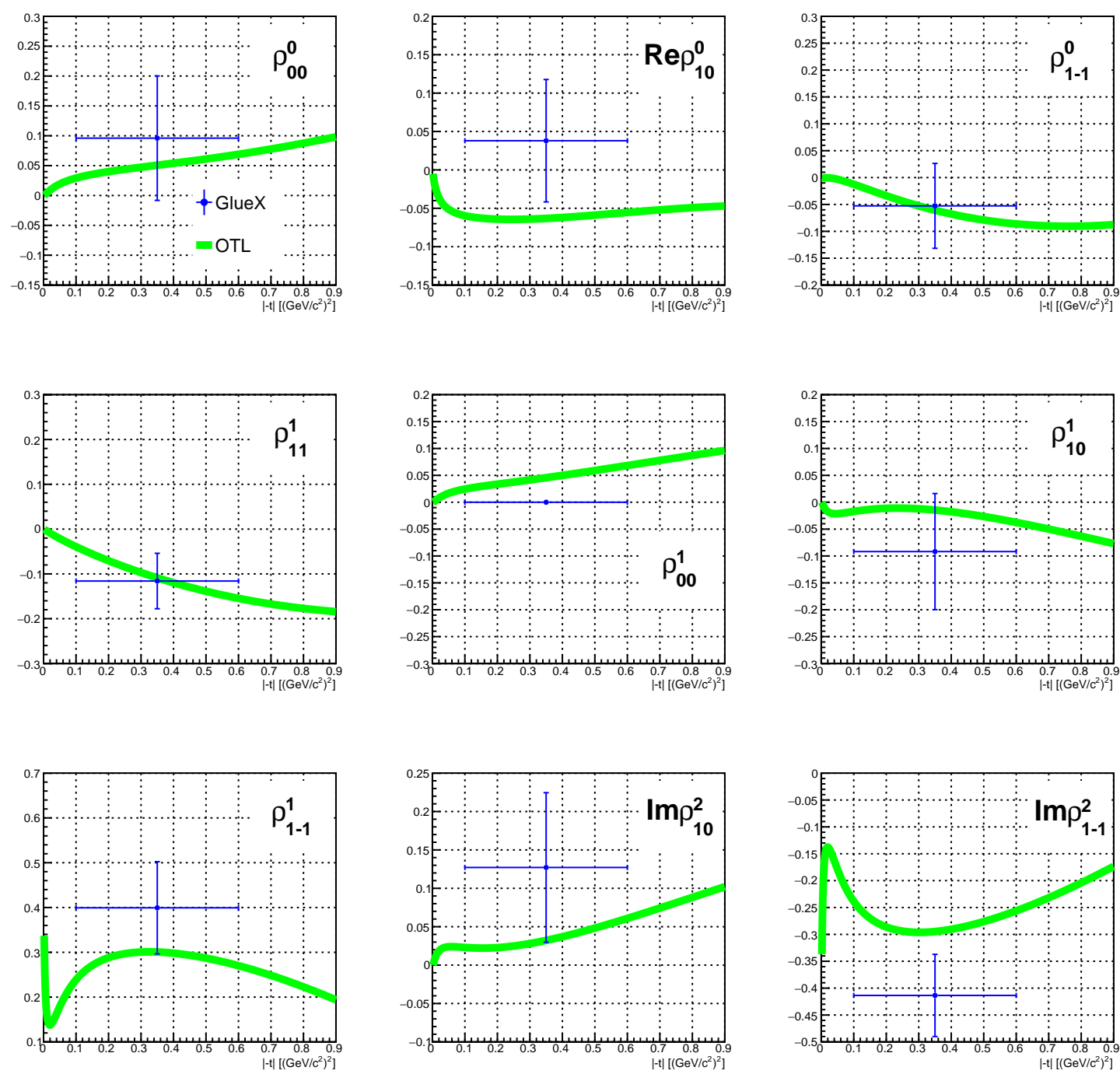

Figure 5.10: Fit results for the measurable SDMEs in the radiative decay channel. Error bars indicate the quadrature addition of statistical errors returned by AmpTools and the systematic uncertainties assigned in Table 5.6. Parameter $\rho_{00}^{1}$ is fixed in the minimization. This result is compared with predictions from the Oh, Titov, Lee model [11]. 

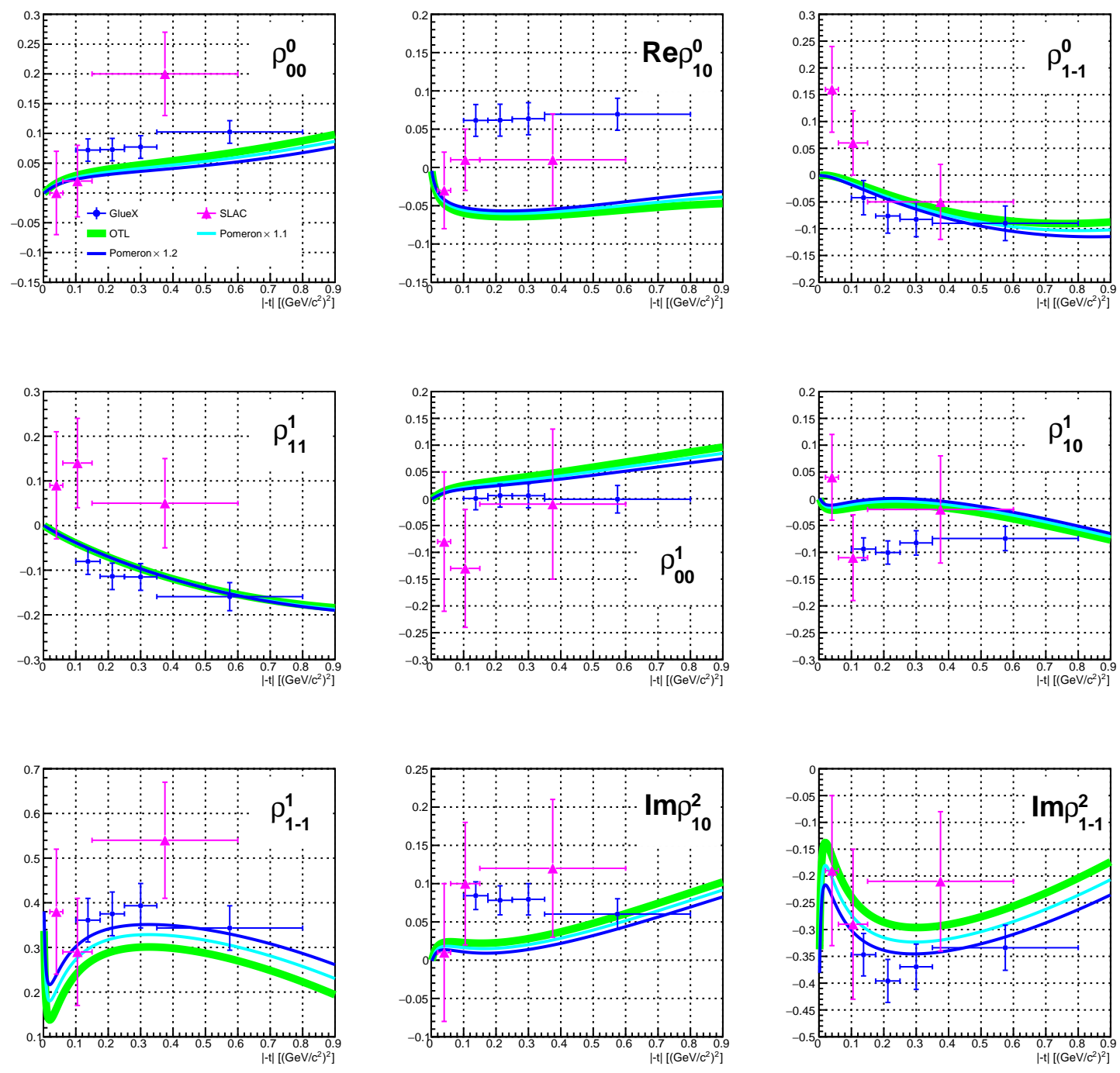

Figure 5.11: Fit results for the measurable SDMEs in the hadronic decay channel showing the effect of varying the Pomeron contribution to the OTL amplitudes. 
These results represent the most precise measurement of the SDMEs in $\omega$ photoproduction at $E_{\gamma}=9 \mathrm{GeV}$ to-date $^{1}$. Even though this represents a two order-of-magnitude increase over the world data in statistics at these energies, the data used in this analysis from Spring 2016 constitutes less than $10 \%$ of the total physics data recorded so far by the GlueX detector, and there are several years of additional running planned. This increased data will allow extending this study to higher t-values where the interplay of the amplitudes leads to more interesting behavior of the SDMEs. Of course, these measurements will continue to improve as our systematic uncertainties are better understood. Even with our current understanding of the results, these measurements can be used to improve existing models of $\omega$ photoproduction. It is clear from this analysis that the rich structure of the experimental data at GlueX and its unique detection capabilities will continue to provide ample opportunities for great advancement in our understanding of QCD processes in photoproduction for years to come.

\footnotetext{
${ }^{1}$ Please note that the results presented in this thesis are considered preliminary until thoroughly vetted by the entire GlueX collaboration.
} 


\section{Bibliography}

[1] D. J. Gross, "The discovery of asymptotic freedom and the emergence of QCD," PNAS, vol. 102, no. $26,2005$.

[2] C. Patrignani et al., "The review of particle physics," Chin. Phys. C, vol. 40, 2016.

[3] C. Meyer and E. Swanson, "Hybrid mesons," Progress in Particle and Nuclear Physics, vol. 82, pp. 21-58, 2015.

[4] J. J. Dudek, R. G. Edwards, P. Guo, and C. E. Thomas, "Toward the excited isoscalar meson spectrum from lattice QCD," Phys. Rev. D, vol. 88, 2013.

[5] D. Amelin et al., "Investigation of hybrid states in the VES experiment at the Institute for High Energy Physics (Protvino)," Phys. of Atomic Nucl., vol. 68, no. 3, pp. 359-371, 2005.

[6] E. Chudakov, "Photoproduction cross section." https://userweb.jlab.org/ gen/gluex/bg_ sim.html. Accessed August 18, 2017.

[7] K. Schilling, P. Seyboth, and G. Wolf, "On the analysis of vector-meson production by polarized photons," Nuclear Physics B, vol. 15, 1970.

[8] F. Halzen and A. D. Martin, Quarks \& Leptons, ch. 6, pp. 132-135. John Wiley \& Sons, Inc., 1984.

[9] C. Meyer, "A review of asymmetry measurements in vector meson photoproduction experiments." GlueX-doc-3076, August 2016.

[10] Q. Zhao, J. Al-Khalili, and P. Cole, "Vector meson photoproduction studied in its radiative decay channel," Phys. Rev. C, vol. 71, no. 054004, 2005.

[11] Y. Oh, A. I. Titov, and T.-S. H. Lee, "Nucleon resonanves in $\omega$ photoproduction," Phys. Rev. $C$, vol. 63 , no. $025201,2001$.

[12] A. Donnachie and P. Landshoff, "Elastic scattering and diffractive dissociation," Nucl. Phys. $B$, vol. 244, pp. 322-336, 1984.

[13] A. I. Titov, T.-S. H. Lee, H. Toki, and O. Streltsova, "Structure of the $\phi$ photoproduction amplitude at a few GeV," Phys. Rev. C, vol. 60, no. 035205, 1999.

[14] M. Williams, "Numerical object oriented quantum field theory calculations," Computer Physics Communications, vol. 180, 2009.

[15] J. Ballam et al., "Vector-meson production by polarized photons at 2.8, 4.7, and $9.3 \mathrm{GeV}$," Phys. Rev. D, vol. 7, no. 11, pp. 3150-3177, 1973.

[16] GlueX Collaboration, "The technical construction report." GlueX-doc-2511, 2016. 
[17] B. Pratt, Diamond Radiator Fabrication, Characterization and Performance for the GlueX Experiment. PhD thesis, University of Connecticut, 2017.

[18] U. Timm, "Coherent bremsstrahlung of electrons in crystals," Fortschritte der Phys., vol. 17, no. $765,1969$.

[19] A. Barnes, Development of the tagger microscope 83 Analysis of Spin Density Matrix Elements in $\gamma p \rightarrow \phi p$ for the GlueX Experiment. PhD thesis, University of Connecticut, 2017.

[20] M. Dugger et al., "Design and construction of a high-energy photon polarimeter," NIM A, vol. 867, pp. 115-127, 2017.

[21] M. Dugger, "TPOL update." GlueX-doc-3133, 2016.

[22] K. Livingston, "Polarization from coherent bremssrahlung enhancement." CLAS Note 2011-020, 2011.

[23] GlueX Collaboration, "Measurement of the beam asymmetery $\Sigma$ for $\pi^{0}$ and $\eta$ photoproduction on the proton at $E_{\gamma}=9 \mathrm{GeV}$," Phys. Rev. C, vol. 95, no. 042201, 2017.

[24] F. Barbosa et al., "Pair spectrometer hodoscope for Hall D at Jefferson Lab," NIM A, vol. 795, pp. 376-380, 2015.

[25] A. Subedi, "FCAL update." GlueX-doc-3307, 2017.

[26] Y. V. Haarlem et al., "The GlueX central drift chamber: Design and performance," NIM A, vol. 622 , pp. 142-156, 2010.

[27] L. Pentchev et al., "Studies with cathode drift chambers for the GlueX experiement at Jefferson Lab," NIM A, vol. 845, pp. 281-284, 2017.

[28] E. Pooser, The GlueX Start counter $\& 3$ Beam asymmetry $\Sigma$ in single $\pi^{0}$ photoproduction. $\mathrm{PhD}$ thesis, Florida International University, 2016.

[29] S. Denisov et al., "Characteristice of the TOF counters for GlueX experiment," NIM A, vol. 494, pp. 495-499, 2002.

[30] GlueX Collaboration, "Hall D/Gluex technical design report." GlueX-doc-2442, 2016.

[31] W. Blum and L. Rolandi, Particle Detection wth Drift Chambers. Springer-Verlag, 1993. pp. $120-122$.

[32] S. Taylor, "Tracking update: Dealing with CDC straw sag." GlueX-doc-2825, 2015.

[33] V. Blobel, "Millepede II - draft manual." http://www.desy.de/ kleinwrt/MP2/doc/html/ draftman_page.html, 2007. Accessed 19 August 2017.

[34] W. R. Inc., "Mathematica, Version 11.1." Champaign, IL, 2017.

[35] "sim-recon." https://github.com/JeffersonLab/sim-recon.

[36] R. Frühwitrh, "Application of Kalman filtering to track and vertex fitting," Nuclear Instruments and Methods A, vol. 262, 1987.

[37] W. Hulsbergen, "The global covariance matrix of tracks fitted with a kalman filter and an application in detector alignment," NIM A, vol. 600, pp. 471-477, 2009.

[38] J. Amotaal et al., "Application of vertex and mass constraints in track-based alignment," NIM A, vol. 712, pp. 48-55, 2013. 
[39] M. Staib, "Solenoid studies." GlueX-doc-2589, October 2014.

[40] P. Mattione, "Least squares kinematic fitting of physics reactions." GlueX-doc-2112, April 2016.

[41] "AmpTools." https://github.com/mashephe/AmpTools. Accessed: 2017-08-03.

[42] R. Mitchell, "A quick overview of the IU AmpTools framework." https://www.jlab.org/div_

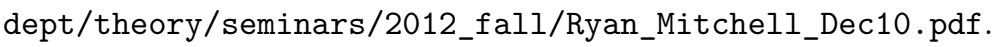

[43] M. Shepherd, "Amplitude analysis." GlueX-doc-2270, 2013.

[44] M. Staib, "Systematic studies for $\omega$ SDMEs." GlueX-doc-3357, August 2017. 


\section{Appendix A}

\section{SDME Fit Results}

In each of the figures that follow, the points are the experimental data, the filled histogram is the re-weighted Monte Carlo, and The accidental background is indicated by the striped component. The fit is performed as described in Section 5.1. The points are the experimental data, the red filled histogram is the contribution from the tagger-accidental background, and the green filled histogram is the re-weighted accepted Monte Carlo. The angle $\Phi$ is the angle of the decay plane relative to the photon polarization vector, and $\cos \theta$ and $\phi$ are the decay angles in the helicity frame as described in Section 1.4.3.

\section{A.1 $\omega \rightarrow \pi^{+} \pi^{-} \pi^{0}$}

$\Phi$

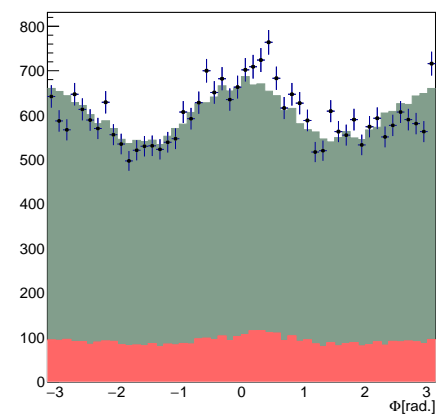

$\cos \theta$

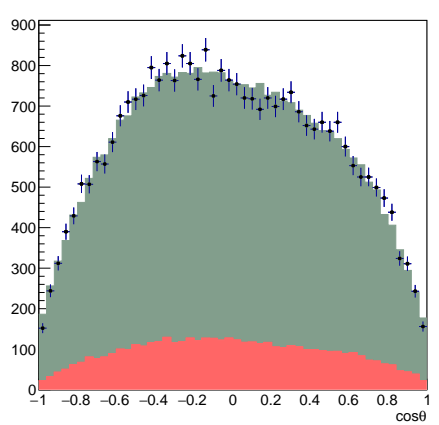

$\phi$

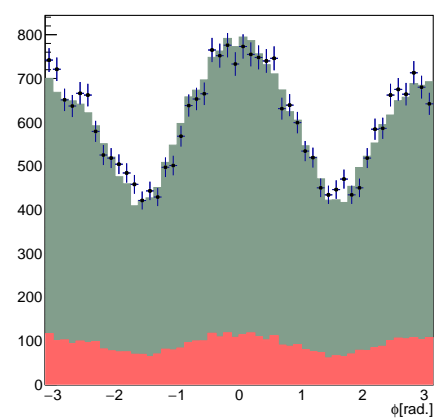

Figure A.1: Comparison of accidental-subtracted data with accepted Monte Carlo re-weighted by the results of the fit for $|t| \in[0.100,0.175)$, PARA polarization, hadronic decay. 

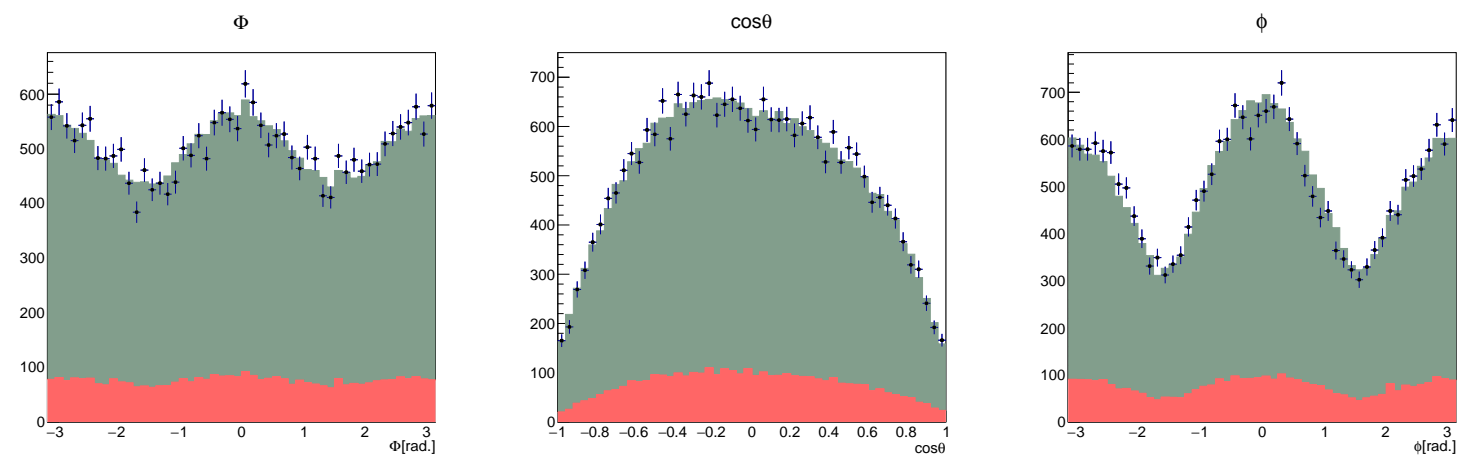

Figure A.2: Comparison of accidental-subtracted data with accepted Monte Carlo re-weighted by the results of the fit for $|t| \in[0.175,0.250)$, PARA polarization, hadronic decay.
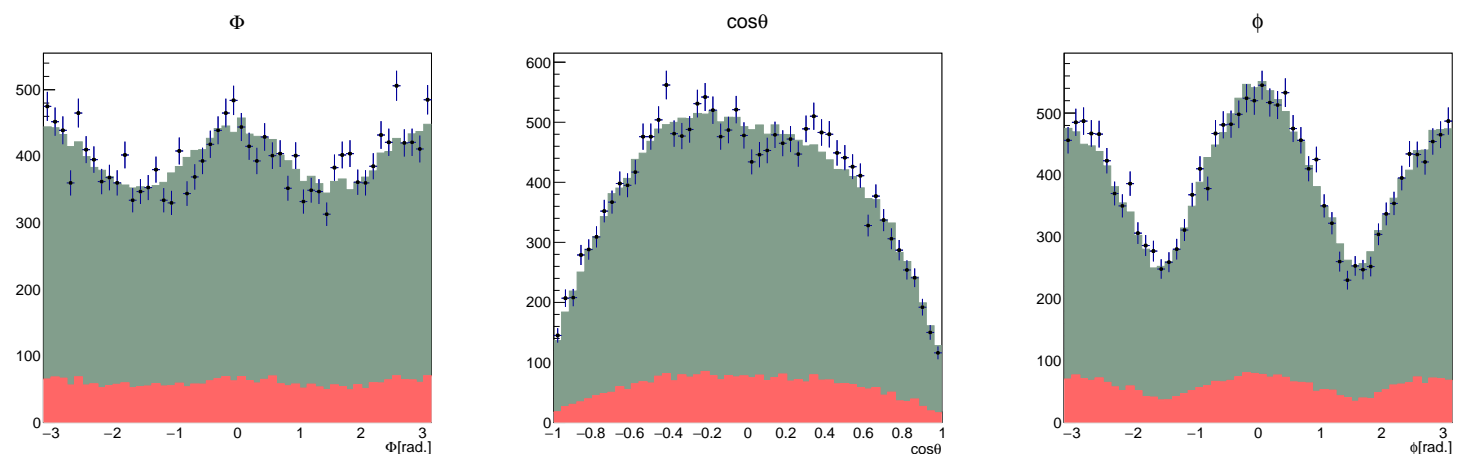

Figure A.3: Comparison of accidental-subtracted data with accepted Monte Carlo re-weighted by the results of the fit for $|t| \in[0.250,0.350)$, PARA polarization, hadronic decay.
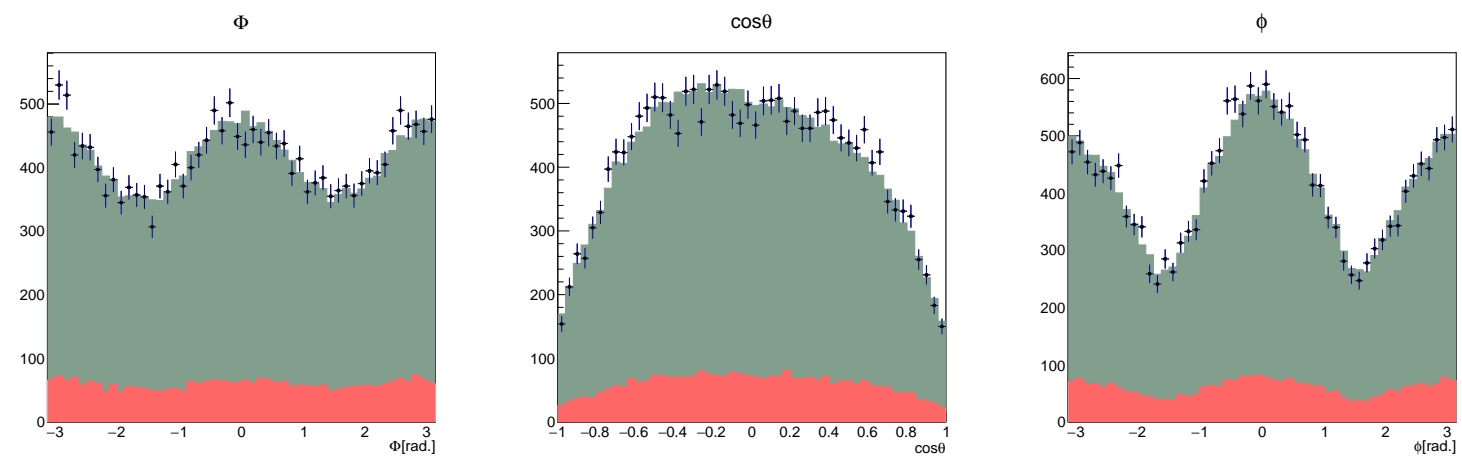

Figure A.4: Comparison of accidental-subtracted data with accepted Monte Carlo re-weighted by the results of the fit for $|t| \in[0.350,0.800)$, PARA polarization, hadronic decay. 

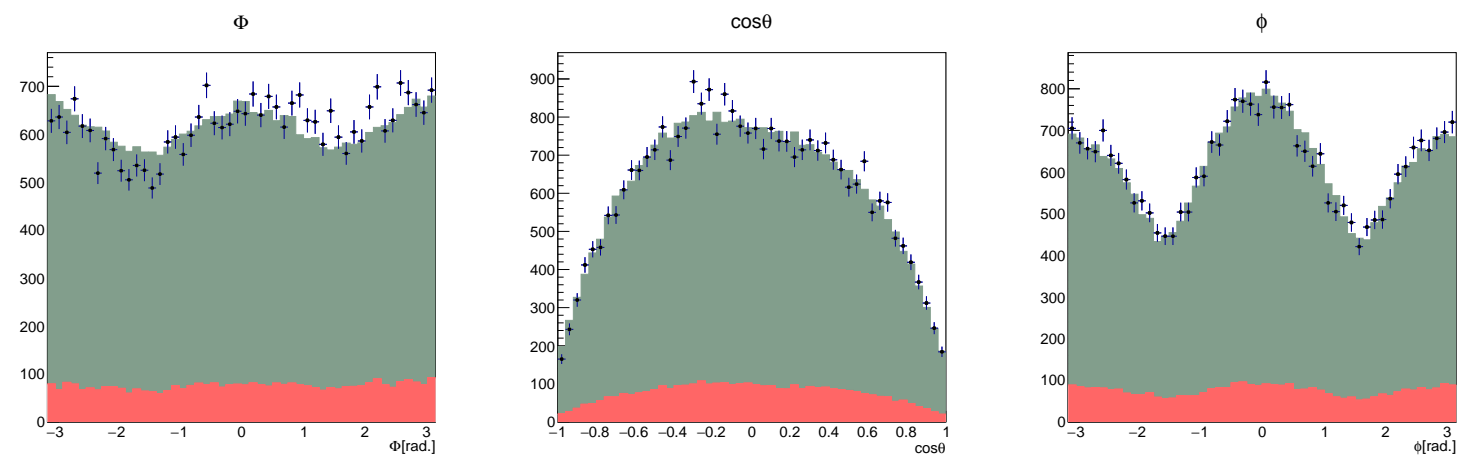

Figure A.5: Comparison of accidental-subtracted data with accepted Monte Carlo re-weighted by the results of the fit for $|t| \in[0.100,0.175)$, PERP polarization, hadronic decay.

$\Phi$

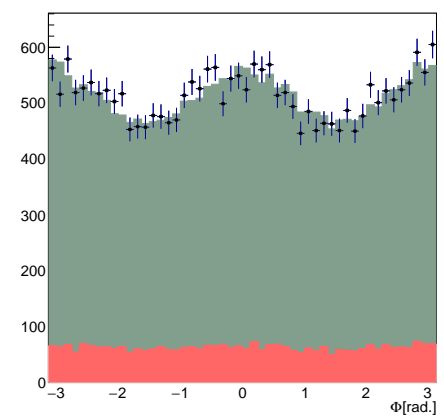

$\cos \theta$

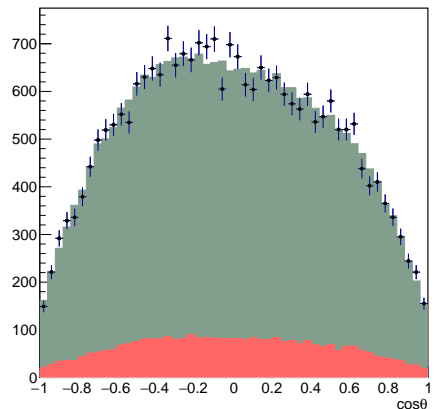

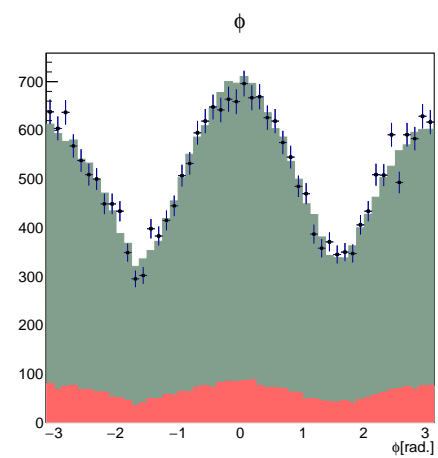

Figure A.6: Comparison of accidental-subtracted data with accepted Monte Carlo re-weighted by the results of the fit for $|t| \in[0.175,0.250)$, PERP polarization, hadronic decay.
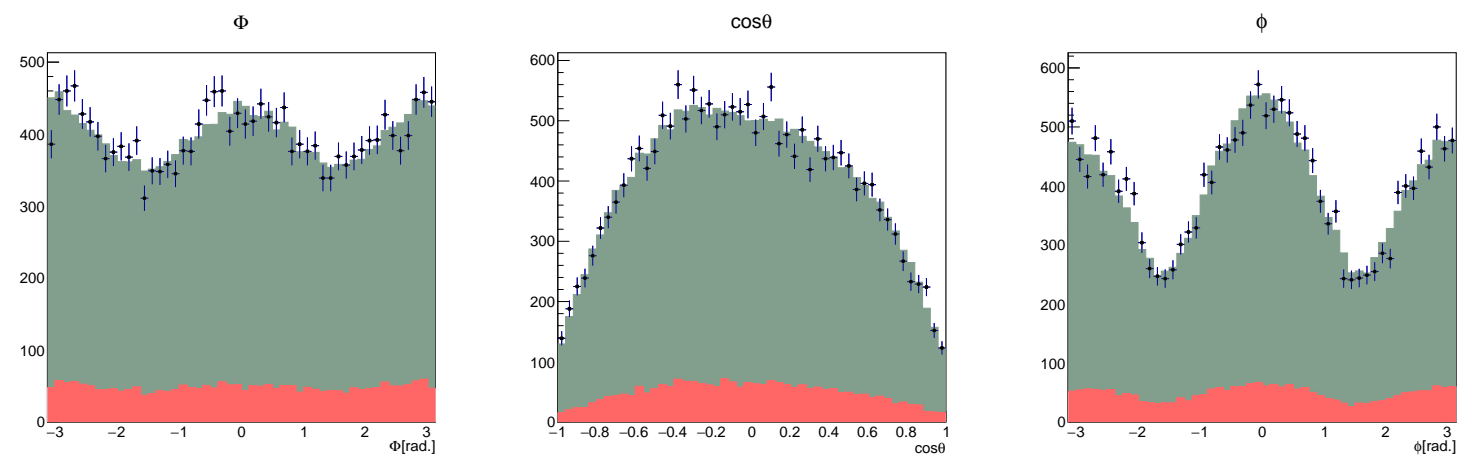

Figure A.7: Comparison of accidental-subtracted data with accepted Monte Carlo re-weighted by the results of the fit for $|t| \in[0.250,0.350)$, PERP polarization, hadronic decay. 

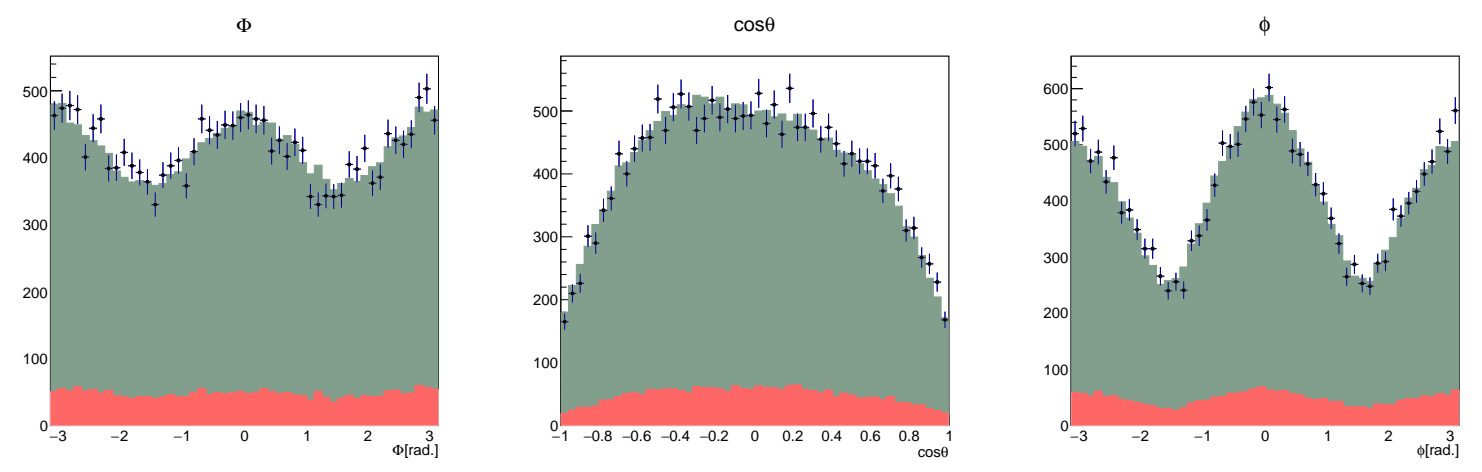

Figure A.8: Comparison of accidental-subtracted data with accepted Monte Carlo re-weighted by the results of the fit for $|t| \in[0.350,0.800)$, PERP polarization, hadronic decay. 
A.2 $\omega \rightarrow \pi^{0} \gamma$
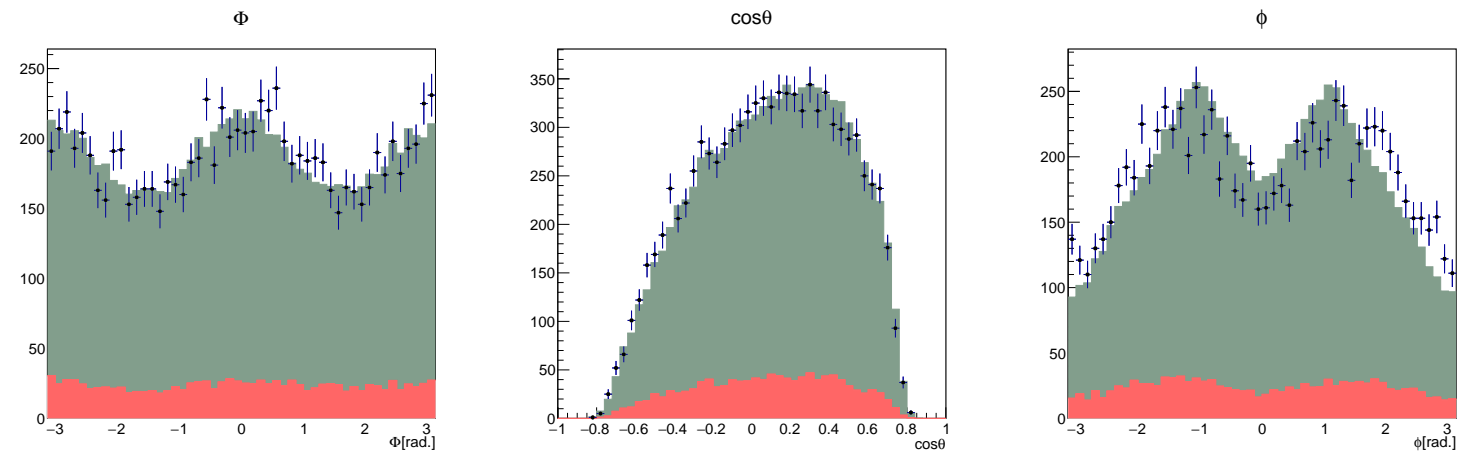

Figure A.9: Comparison of accidental-subtracted data with accepted Monte Carlo re-weighted by the results of the fit for $|t| \in[0.100,0.600)$, PARA polarization, radiative decay.
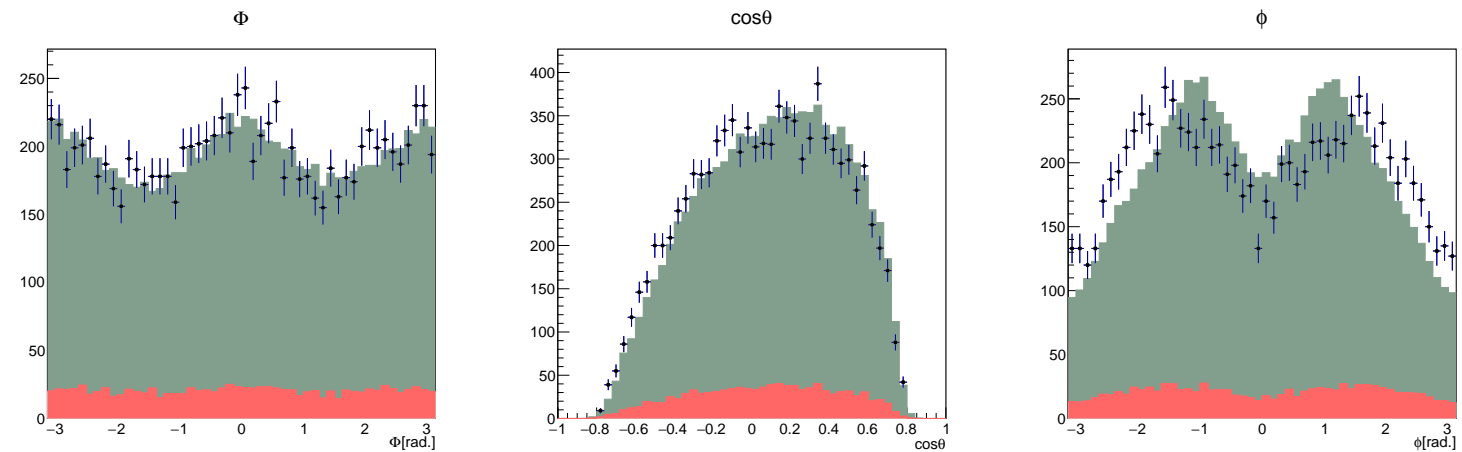

Figure A.10: Comparison of accidental-subtracted data with accepted Monte Carlo re-weighted by the results of the fit for $|t| \in[0.100,0.600)$, PERP polarization, radiative decay. 


\section{Appendix B}

\section{Table of Systematic Uncertainties}

In this appendix, we present each of the individual values for systematic uncertainties assigned using the method described in section 5.4. For additional details, see reference [44].

\begin{tabular}{|c|c|c|c|c|c|c|c|c|c|}
\hline Varied Parameter & \multicolumn{9}{|c|}{ Kinematic Fit CL Lower Bound $\in[0.00,0.10]$} \\
\hline Dataset & $\sigma \rho_{00}^{0}$ & $\sigma \rho_{10}^{0}$ & $\sigma \rho_{1-1}^{0}$ & $\sigma \rho_{11}^{1}$ & $\sigma \rho_{00}^{1}$ & $\sigma \rho_{10}^{1}$ & $\sigma \rho_{1-1}^{1}$ & $\sigma \rho_{10}^{2}$ & $\sigma \rho_{1-}^{2}$ \\
\hline Hadronic Decay & & 0001 & 0.002 & 0.004 & & & 0.002 & & \\
\hline $\mathrm{Had}$ & & & & 09 & & & 003 & & 02 \\
\hline & & & & & & & 4 & & \\
\hline ive $\mathrm{De}$ & & & 006 & .006 & IIxed & 0.018 & 0.010 & .007 & \\
\hline Varied Para & \multicolumn{9}{|c|}{ Missing Mass Squared Lower Bound $\in\left[-0.100,-0.010 \mathrm{GeV}^{2}\right]$} \\
\hline Dataset & $\begin{array}{l}0 \\
00\end{array}$ & $\sigma \rho_{10}^{0}$ & $\sigma \rho_{1-1}^{0}$ & $\sigma \Omega^{1}$ & $\sigma \rho_{00}^{1}$ & $\sigma \rho_{10}^{1}$ & $\sigma \rho_{1}^{1}$ & $\sigma \rho_{10}^{2}$ & $1-$ \\
\hline iic Dec & & & & & 000 & 01 & 0.001 & & \\
\hline & & & & & & & 0 & & \\
\hline & & & & & & & 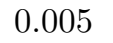 & & \\
\hline $\operatorname{Rad}$ & & 02 & 000 & 0.000 & IIxed & 0.010 & 0.008 & .001 & \\
\hline ed Par & \multicolumn{9}{|c|}{ Missing Mass Squared Upper Bound $\in\left[0.010,0.100 \mathrm{GeV}^{2}\right]$} \\
\hline Data: & & & $\sigma \rho_{1-1}^{0}$ & $\sigma \rho_{11}^{1}$ & $\sigma \rho_{00}^{1}$ & $\sigma \rho_{10}^{1}$ & $\sigma \rho_{1}^{1}$ & $\sigma \rho_{10}^{2}$ & $1-1$ \\
\hline $\mathrm{H}$ & & & & & & & 0.002 & & \\
\hline & & & & & & & 0.00 & & 00 \\
\hline & & & & & & r & 0.002 & & 00 \\
\hline Radiative Dec & 12 & 0.002 & 0.000 & 0.003 & fixed & 0.005 & 0.000 & 0.001 & 001 \\
\hline Varied Par & \multicolumn{9}{|c|}{ Missing Energy Lower Bound $\in[-1.5,-0.5 \mathrm{GeV}]$} \\
\hline$\overline{\text { Datas }}$ & 00 & $0_{10}^{0}$ & $\sigma \rho_{1-1}^{0}$ & $\sigma \rho_{11}^{1}$ & $\sigma \rho_{00}^{1}$ & $\sigma \rho_{10}^{1}$ & $\sigma \rho_{1-1}^{1}$ & $\sigma \rho_{10}^{2}$ & $\sigma \rho_{1-1}^{2}$ \\
\hline iic De & 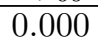 & 0.000 & 0.000 & 0.000 & 0.000 & 0.000 & 0.000 & 0.000 & 0.000 \\
\hline & & & 00 & 0 & 0. & 000 & 0.000 & 000 & .000 \\
\hline $\mathrm{A}$ & & & 2 & 002 & fix & 0.008 & 0.003 & 0.001 & 0.001 \\
\hline Radiative Decay PERP & 005 & 0.003 & 0.002 & 0.001 & fixed & 0.001 & 0.001 & 0.000 & 0.001 \\
\hline Varied Para & \multicolumn{9}{|c|}{ Missing Energy Upper Bound $\in[0.5,1.5 \mathrm{GeV}]$} \\
\hline Data & & & $\sigma \rho_{1-1}^{0}$ & $\sigma \rho_{11}^{1}$ & $\sigma \rho_{00}^{1}$ & $\sigma \rho_{10}^{1}$ & $\sigma \rho_{1}^{1}$ & $2_{10}^{2}$ & $\sigma \rho_{1-}^{2}$ \\
\hline nic De & 002 & 0.002 & 0.001 & 0.001 & 0.001 & 0.001 & 0.001 & 0.001 & 0.000 \\
\hline $\mathrm{De}$ & & & & 2 & 0.001 & 0.001 & 0.003 & 02 & 001 \\
\hline$R A$ & & & & & & 0.002 & 0.009 & 02 & 0.000 \\
\hline Radiative Decay PERP & 0.031 & 0.015 & 0.007 & 0.002 & fixed & 0.006 & 0.011 & 0.005 & 0.010 \\
\hline
\end{tabular}




\begin{tabular}{|c|c|c|c|c|c|c|c|c|c|}
\hline Varied Parameter & \multicolumn{9}{|c|}{ Missing Transverse Momentum Upper Bound $\in[0.15,0.35 \mathrm{GeV}]$} \\
\hline Dataset & $\sigma \rho_{00}^{0}$ & $\sigma \rho_{10}^{0}$ & $\sigma \rho_{1-1}^{0}$ & $\sigma \rho_{11}^{1}$ & $\sigma \rho_{00}^{1}$ & $\sigma \rho_{10}^{1}$ & $\sigma \rho_{1-1}^{1}$ & $\sigma \rho_{10}^{2}$ & $\sigma \rho_{1-1}^{2}$ \\
\hline Hadronic Decay PARA & 0.000 & 0.000 & 0.000 & 0.000 & 0.000 & 0.000 & 0.001 & 0.000 & 0.001 \\
\hline Hadronic Decay PERP & 0.000 & 0.000 & 0.000 & 0.000 & 0.000 & 0.000 & 0.001 & 0.000 & 0.001 \\
\hline Radiative Decay PARA & 0.000 & 0.000 & 0.000 & 0.000 & fixed & 0.001 & 0.000 & 0.000 & 0.000 \\
\hline Radiative Decay PERP & 0.001 & 0.000 & 0.000 & 0.000 & fixed & 0.001 & 0.000 & 0.000 & 0.000 \\
\hline Varied Parameter & \multicolumn{9}{|c|}{$\pi^{0}$ Measured Inv. Mass Lower Bound $\in[0.110,0.125 \mathrm{GeV}]$} \\
\hline Dataset & $\sigma \rho_{00}^{0}$ & $\sigma \rho_{10}^{0}$ & $\sigma \rho_{1-1}^{0}$ & $\sigma \rho_{11}^{1}$ & $\sigma \rho_{00}^{1}$ & $\sigma \rho_{10}^{1}$ & $\sigma \rho_{1-1}^{1}$ & $\sigma \rho_{10}^{2}$ & $\sigma \rho_{1-1}^{2}$ \\
\hline Hadronic Decay PARA & 0.000 & 0.000 & 0.001 & 0.001 & 0.000 & 0.000 & 0.002 & 0.001 & 0.002 \\
\hline Hadronic Decay PERP & 0.000 & 0.000 & 0.001 & 0.001 & 0.001 & 0.001 & 0.003 & 0.000 & 0.001 \\
\hline Radiative Decay PARA & 0.006 & 0.002 & 0.003 & 0.006 & fixed & 0.005 & 0.006 & 0.003 & 0.003 \\
\hline Radiative Decay PERP & 0.005 & 0.001 & 0.002 & 0.002 & fixed & 0.006 & 0.005 & 0.005 & 0.004 \\
\hline Varied Parameter & \multicolumn{9}{|c|}{$\pi^{0}$ Measured Inv. Mass Upper Bound $\in[0.145,0.155 \mathrm{GeV}]$} \\
\hline Dataset & $\sigma \rho_{00}^{0}$ & $\sigma \rho_{10}^{0}$ & $\sigma \rho_{1-1}^{0}$ & $\sigma \rho_{11}^{1}$ & $\sigma \rho_{00}^{1}$ & $\sigma \rho_{10}^{1}$ & $\sigma \rho_{1-1}^{1}$ & $\sigma \rho_{10}^{2}$ & $\sigma \rho_{1-1}^{2}$ \\
\hline Hadronic Decay PARA & 0.000 & 0.001 & 0.001 & 0.002 & 0.001 & 0.001 & 0.003 & 0.000 & 0.003 \\
\hline Hadronic Decay PERP & 01 & 00 & 0 & 1 & 00 & & & 01 & 02 \\
\hline Radiative Decay PARA & 0.005 & 0.004 & 0.002 & 0.003 & fixed & 0.009 & 0.003 & 0.003 & 0.006 \\
\hline Radiative Decay PERP & 0.013 & 0.005 & 0.002 & 0.003 & fixed & 0.008 & 0.002 & 0.005 & 0.004 \\
\hline Varied Parameter & \multicolumn{9}{|c|}{$\omega$ w Kin. Fit Inv. Mass Lower Bound $\in[0.745,0.775 \mathrm{GeV}]$} \\
\hline Dataset & $\sigma \rho_{00}^{0}$ & $\sigma \rho_{10}^{0}$ & $\sigma \rho_{1-1}^{0}$ & $\sigma \rho_{11}^{1}$ & $\sigma \rho_{00}^{1}$ & $\sigma \rho_{10}^{1}$ & $\sigma \rho_{1-1}^{1}$ & $\sigma \rho_{10}^{2}$ & $\sigma \rho_{1-1}^{2}$ \\
\hline Hadronic Decay PARA & 0.003 & 0.001 & 0.001 & 0.003 & 0.001 & 0.001 & 0.004 & 0.002 & 0.003 \\
\hline Hadronic Decay PERP & 0.004 & 0.001 & 0.000 & 0.007 & 0.003 & 0.001 & 0.001 & 0.003 & 0.001 \\
\hline Radiative Dece & 0.014 & 0.009 & 0.010 & 0.004 & fixed & 010 & 0.025 & .004 & 0.007 \\
\hline Radiative Decay PERP & 22 & 01 & 01 & 8 & fixed & 13 & 10 & 11 & 0.032 \\
\hline ed Parc & \multicolumn{9}{|c|}{$\omega$ Kin. Fit Inv. Mass Upper Bound $\in[0.795,0.825 \mathrm{GeV}]$} \\
\hline Dataset & $\sigma \rho_{00}^{0}$ & $\sigma \rho_{10}^{0}$ & $\sigma \rho_{1-1}^{0}$ & $\sigma \rho_{11}^{1}$ & $\sigma \rho_{00}^{1}$ & $\sigma \rho_{10}^{1}$ & $\sigma \rho_{1-1}^{1}$ & $\sigma \rho_{10}^{2}$ & $\sigma \rho_{1-1}^{2}$ \\
\hline Hadronic Decay & & & & 0.004 & 0.002 & & & & 0.003 \\
\hline Hadronic Dece & 002 & 000 & 0.001 & 0.002 & 0.000 & 0.001 & 0.002 & 0.001 & 0.003 \\
\hline Radiative Dec & 0.024 & 0.006 & 0.004 & 0.004 & fixed & 0.010 & 0.007 & 0.013 & 0.004 \\
\hline Radiative Decay PERP & 0.014 & 0.005 & 0.004 & 0.008 & fixed & 0.008 & 0.012 & 0.005 & 0.004 \\
\hline Varied Parameter & \multicolumn{9}{|c|}{ Minimum Photon Beam Energy $\in[8.35,8.45 \mathrm{GeV}]$} \\
\hline Dataset & $\sigma \rho_{00}^{0}$ & $\sigma \rho_{10}^{0}$ & $\sigma \rho_{1-1}^{0}$ & $\sigma \rho_{11}^{1}$ & $\sigma \rho_{00}^{1}$ & $\sigma \rho_{10}^{1}$ & $\sigma \rho_{1-1}^{1}$ & $\sigma \rho_{10}^{2}$ & $\sigma \rho_{1-1}^{2}$ \\
\hline Hadronic Decay PARA & 0.000 & 0.000 & 0.001 & 0.002 & 0.001 & 0.001 & 0.005 & 0.002 & 0.001 \\
\hline Hadronic Deca & 00 & 00 & 00 & 0.000 & 0.001 & 003 & 02 & 0.001 & 0.004 \\
\hline Radiative Dec & .009 & .003 & 0.003 & 0.006 & fixed & 0.007 & 0.011 & 0.006 & 0.003 \\
\hline Radiative Deca & 0.009 & 0.002 & 0.002 & 0.002 & fixed & 0.001 & 0.007 & 0.002 & 0.009 \\
\hline Varied Parameter & \multicolumn{9}{|c|}{ Maximum Photon Beam Energy $\in[8.95,9.05 \mathrm{GeV}]$} \\
\hline Dataset & $\sigma \rho_{00}^{0}$ & $\sigma \rho_{10}^{0}$ & $\sigma \rho_{1-1}^{0}$ & $\sigma \rho_{11}^{1}$ & $\sigma \rho_{00}^{1}$ & $\sigma \rho_{10}^{1}$ & $\sigma \rho_{1-1}^{1}$ & $\sigma \rho_{10}^{2}$ & $\sigma \rho_{1-1}^{2}$ \\
\hline Hadronic Deca & 0.000 & 0.000 & 0.001 & 0.001 & 0.001 & 0.001 & 0.002 & 0.000 & 0.003 \\
\hline Hadronic Decay PERP & 0.000 & 0.000 & 0.001 & 0.002 & 0.000 & 0.001 & 0.004 & 0.001 & 0.002 \\
\hline Radiative Decay PARA & 0.005 & 0.003 & 0.002 & 0.005 & fixed & 0.003 & 0.006 & 0.003 & 0.005 \\
\hline Radiative Decay PERP & 0.013 & 0.006 & 0.001 & 0.006 & fixed & 0.023 & 0.003 & 0.009 & 0.005 \\
\hline ed Parameter & \multicolumn{9}{|c|}{ Proton Momentum Lower Bound $\in[0.05,0.50 \mathrm{GeV}]$} \\
\hline Dataset & $\sigma \rho_{00}^{0}$ & $\sigma \rho_{10}^{0}$ & $\sigma \rho_{1-1}^{0}$ & $\sigma \rho_{11}^{1}$ & $\sigma \rho_{00}^{1}$ & $\sigma \rho_{10}^{1}$ & $\sigma \rho_{1-1}^{1}$ & $\sigma \rho_{10}^{2}$ & $\sigma \rho_{1-1}^{2}$ \\
\hline Hadronic Decay PARA & 0.006 & 0.005 & 0.007 & 0.007 & 0.002 & 0.003 & 0.014 & 0.003 & 0.005 \\
\hline Hadronic Decay PERP & 0.005 & 0.006 & 0.011 & 0.009 & 0.004 & 0.003 & 0.003 & 0.004 & 0.010 \\
\hline Radiative Decay PARA & 0.058 & 0.051 & 0.072 & 0.014 & fixed & 0.037 & 0.033 & 0.022 & 0.031 \\
\hline Radiative Decay PERP & 0.041 & 0.055 & 0.070 & 0.007 & fixed & 0.022 & 0.015 & 0.005 & 0.018 \\
\hline
\end{tabular}




\begin{tabular}{|c|c|c|c|c|c|c|c|c|c|}
\hline Varied Parameter & \multicolumn{9}{|c|}{ Pion Momentum Lower Bound $\in[0.05,0.50 \mathrm{GeV}]$} \\
\hline Dataset & $\sigma \rho_{00}^{0}$ & $\sigma \rho_{10}^{0}$ & $\sigma \rho_{1-}^{0}$ & $\sigma \rho_{11}^{1}$ & $\sigma \rho_{00}^{1}$ & $\sigma \rho_{10}^{1}$ & $\sigma \rho_{1-1}^{1}$ & $\sigma \rho_{10}^{2}$ & $\sigma \rho_{1}^{2}$ \\
\hline Hadronic Decay PARA & 001 & 0.000 & 0.001 & 000 & 0.000 & 0.000 & 0.000 & 0.000 & 0002 \\
\hline Hadronic Deca & 00 & $0 .($ & 0.001 & .000 & 0.000 & 0.000 & 0.001 & .000 & 0.002 \\
\hline Varied Parameter & \multicolumn{9}{|c|}{ Pion $\theta$ Lower Bound $\in[1.0,5.0$ deg. $]$} \\
\hline Dataset & $\sigma \rho_{00}^{0}$ & $\sigma \rho_{10}^{0}$ & $\sigma \rho_{1-1}^{0}$ & $\sigma \rho_{11}^{1}$ & $\sigma \rho_{00}^{1}$ & $\sigma \rho_{10}^{1}$ & $\sigma \rho_{1-1}^{1}$ & $\sigma \rho_{10}^{2}$ & $\sigma \rho_{1-}^{2}$ \\
\hline Hadronic Decay PARA & 0.016 & 0.020 & 0.029 & 0.012 & 0.013 & 0.011 & 0.028 & 0.007 & 0.015 \\
\hline Hadronic Decay PERP & 0.016 & 0.018 & 0.029 & 0.009 & 0.007 & 0.016 & 0.019 & 0.008 & 0.009 \\
\hline Varied Parameter & \multicolumn{9}{|c|}{ Photon $Z_{B C A L}$ Lower Bound $\in[180.0,400.0 \mathrm{~cm}]$} \\
\hline Dataset & $\sigma \rho_{00}^{0}$ & $\sigma \rho_{10}^{0}$ & $\sigma \rho_{1-1}^{0}$ & $\sigma \rho_{11}^{1}$ & $\sigma \rho_{00}^{1}$ & $\sigma \rho_{10}^{1}$ & $\sigma \rho_{1-1}^{1}$ & $\sigma \rho_{10}^{2}$ & $\sigma \rho_{1-1}^{2}$ \\
\hline Hadronic Decay PARA & 0.001 & 0.002 & 0.002 & 0.003 & 0.002 & 0.002 & 0.002 & 0.002 & 0.003 \\
\hline Hadronic Dec & 001 & 0.002 & 0.001 & .001 & 0.002 & .001 & .003 & 001 & .005 \\
\hline Radiative De & 49 & 0.028 & 0.002 & 03 & fixed & .010 & .007 & 0.015 & 0.012 \\
\hline Radiative Decay PERP & 044 & 0.026 & 0.003 & 0.015 & fixed & 0.032 & 0.017 & 0.024 & 0.009 \\
\hline Varied Par & \multicolumn{9}{|c|}{ Photon $Z_{B C A L}$ Upper Bound $\in[360.0,400.0 \mathrm{~cm}]$} \\
\hline Dataset & $\sigma \rho_{00}^{0}$ & $\sigma \rho_{10}^{0}$ & $\sigma \rho_{1-1}^{0}$ & $\sigma \rho_{11}^{1}$ & $\sigma \rho_{00}^{1}$ & $\sigma \rho_{10}^{1}$ & $\sigma \rho_{1-1}^{1}$ & $\sigma \rho_{10}^{2}$ & $\sigma \rho_{1-1}^{2}$ \\
\hline Hadronic De & 000 & 0.000 & 0.001 & 0.001 & 0.001 & .001 & 0.001 & 001 & 0.001 \\
\hline ERP & 01 & 00 & & & 01 & 001 & .001 & 01 & 001 \\
\hline ARA & 000 & 000 & 000 & & fixed & .000 & 0.000 & 0.000 & 0.000 \\
\hline Radiative Decay PERP & 000 & 0.000 & 0.000 & 0.000 & fixed & 0.000 & 0.000 & 0.000 & 0.000 \\
\hline Varied Pa & \multicolumn{9}{|c|}{ Photon $R_{F C A L}$ Lower Bound $\in[15.0,25.0 \mathrm{~cm}]$} \\
\hline Dataset & $\sigma \rho_{00}^{0}$ & $\sigma \rho_{10}^{0}$ & $\sigma \rho_{1-1}^{0}$ & $\sigma \rho_{11}^{1}$ & $\sigma \rho_{00}^{1}$ & $\sigma \rho_{10}^{1}$ & $\sigma \rho_{1-1}^{1}$ & $\sigma \rho_{10}^{2}$ & $\sigma \rho_{1-}^{2}$ \\
\hline nic De & 002 & 03 & 03 & & 01 & 001 & 0.002 & 03 & 3 \\
\hline $\mathrm{RP}$ & 01 & 03 & 3 & & 001 & 001 & .003 & 001 & 002 \\
\hline RA & 22 & 033 & 23 & & fixed & 0.016 & 0.016 & 013 & .016 \\
\hline Radiative Dec & 019 & 0.033 & 0.024 & 0.017 & fixed & 0.028 & 0.017 & 0.021 & 0.010 \\
\hline Varied Par & \multicolumn{9}{|c|}{ Photon $R_{F C A L}$ Upper Bound $\in[92.0,110.0 \mathrm{~cm}]$} \\
\hline Data & $\rho_{00}^{0}$ & $\rho_{10}^{0}$ & $\sigma \rho_{1-1}^{0}$ & $\sigma \rho_{11}^{1}$ & $\sigma \rho_{00}^{1}$ & $\sigma \rho_{10}^{1}$ & $\sigma \rho_{1-1}^{1}$ & $\sigma \rho_{10}^{2}$ & $\sigma \rho_{1-1}^{2}$ \\
\hline iic De & 00 & 00 & 0.001 & 0.002 & 0.002 & 0.001 & 0.001 & 0.001 & 0.002 \\
\hline ERP & 01 & 00 & 000 & 0.001 & 0.002 & 0.001 & 0.001 & 0.001 & 0.001 \\
\hline RA & 2 & .009 & 0.005 & 0.006 & fixed & 0.010 & 0.007 & 0.020 & 0.007 \\
\hline Radiative Decay PERP & 033 & 0.011 & 0.012 & 0.009 & fixed & 0.017 & 0.020 & 0.014 & 0.018 \\
\hline Varied Parameter & \multicolumn{9}{|c|}{ Shower $E_{F C A L}$ Lower Bound $\in[0.100,0.500 \mathrm{GeV}]$} \\
\hline Dataset & $\overline{\sigma \rho} \rho_{00}^{0}$ & $\sigma \rho_{10}^{0}$ & $\sigma \rho_{1-1}^{0}$ & $\sigma \rho_{11}^{1}$ & $\sigma \rho_{00}^{1}$ & $\sigma \rho_{10}^{1}$ & $\sigma \rho_{1-}^{1}$ & $\sigma \rho_{10}^{2}$ & $\sigma \rho_{1-}^{2}$ \\
\hline iic $\mathrm{De}$ & 000 & 0.000 & 0.001 & 0.001 & 0.002 & 0.001 & 0.005 & 0.001 & 0.003 \\
\hline ERP & 000 & 0.000 & 0.003 & 0.001 & 0.002 & 0.001 & 0.004 & 0.001 & 0.004 \\
\hline PARA & 020 & 0.011 & 0.002 & 0.004 & fixed & 0.010 & 0.004 & 0.011 & 0.008 \\
\hline Radiative Decay PERP & 0.019 & 0.009 & 0.006 & 0.016 & fixed & 0.030 & 0.018 & 0.005 & 0.010 \\
\hline
\end{tabular}




\begin{tabular}{|c|c|c|c|c|c|c|c|c|c|}
\hline Varied Parameter & \multicolumn{7}{|c|}{ Shower $E_{B C A L}$ Lower Bound $\in[0.100,0.500 \mathrm{GeV}]$} \\
\hline Dataset & $\sigma \rho_{00}^{0}$ & $\sigma \rho_{10}^{0}$ & $\sigma \rho_{1-1}^{0}$ & $\sigma \rho_{11}^{1}$ & $\sigma \rho_{00}^{1}$ & $\sigma \rho_{10}^{1}$ & $\sigma \rho_{1-1}^{1}$ & $\sigma \rho_{10}^{2}$ & $\sigma \rho_{1-1}^{2}$ \\
\hline Hadronic Decay PARA & 0.001 & 0.001 & 0.000 & 0.001 & 0.002 & 0.002 & 0.002 & 0.002 & 0.002 \\
Hadronic Decay PERP & 0.001 & 0.001 & 0.001 & 0.001 & 0.001 & 0.002 & 0.002 & 0.002 & 0.003 \\
Radiative Decay PARA & 0.000 & 0.000 & 0.000 & 0.000 & fixed & 0.000 & 0.000 & 0.000 & 0.000 \\
Radiative Decay PERP & 0.000 & 0.000 & 0.000 & 0.000 & fixed & 0.000 & 0.000 & 0.000 & 0.000 \\
\hline \hline Varied Parameter & \multicolumn{8}{|c|}{ Degree of Polarization PARA $\in[0.351,0.423]$} \\
\hline Dataset & $\sigma \rho_{00}^{0}$ & $\sigma \rho_{10}^{0}$ & $\sigma \rho_{1-1}^{0}$ & $\sigma \rho_{11}^{1}$ & $\sigma \rho_{00}^{1}$ & $\sigma \rho_{10}^{1}$ & $\sigma \rho_{1-1}^{1}$ & $\sigma \rho_{10}^{2}$ & $\sigma \rho_{1-1}^{2}$ \\
\hline Hadronic Decay PARA & 0.000 & 0.000 & 0.000 & 0.008 & 0.000 & 0.006 & 0.023 & 0.005 & 0.024 \\
Radiative Decay PARA & 0.000 & 0.000 & 0.000 & 0.008 & fixed & 0.008 & 0.023 & 0.004 & 0.027 \\
\hline \hline Varied Parameter & \multicolumn{8}{|c|}{ Degree of Polarization PERP $\in[0.342,0.414]$} \\
\hline Dataset & $\sigma \rho_{00}^{0}$ & $\sigma \rho_{10}^{0}$ & $\sigma \rho_{1-1}^{0}$ & $\sigma \rho_{11}^{1}$ & $\sigma \rho_{00}^{1}$ & $\sigma \rho_{10}^{1}$ & $\sigma \rho_{1-1}^{1}$ & $\sigma \rho_{10}^{2}$ & $\sigma \rho_{1-1}^{2}$ \\
\hline Hadronic Decay PERP & 0.000 & 0.000 & 0.000 & 0.006 & 0.000 & 0.006 & 0.027 & 0.006 & 0.026 \\
Radiative Decay PERP & 0.000 & 0.000 & 0.000 & 0.008 & fixed & 0.004 & 0.031 & 0.013 & 0.029 \\
\hline \hline Varied Parameter & \multicolumn{8}{|c|}{ Fixed $\rho_{00}^{1}$ Value $\in[-0.050,0.050]$} \\
\hline Dataset & $\sigma \rho_{00}^{0}$ & $\sigma \rho_{10}^{0}$ & $\sigma \rho_{1-1}^{0}$ & $\sigma \rho_{11}^{1}$ & $\sigma \rho_{00}^{1}$ & $\sigma \rho_{10}^{1}$ & $\sigma \rho_{1-1}^{1}$ & $\sigma \rho_{10}^{2}$ & $\sigma \rho_{1-1}^{2}$ \\
\hline Radiative Decay PARA & 0.001 & 0.000 & 0.000 & 0.031 & fixed & 0.013 & 0.003 & 0.000 & 0.000 \\
Radiative Decay PERP & 0.001 & 0.000 & 0.000 & 0.031 & fixed & 0.013 & 0.003 & 0.000 & 0.000 \\
\hline
\end{tabular}

